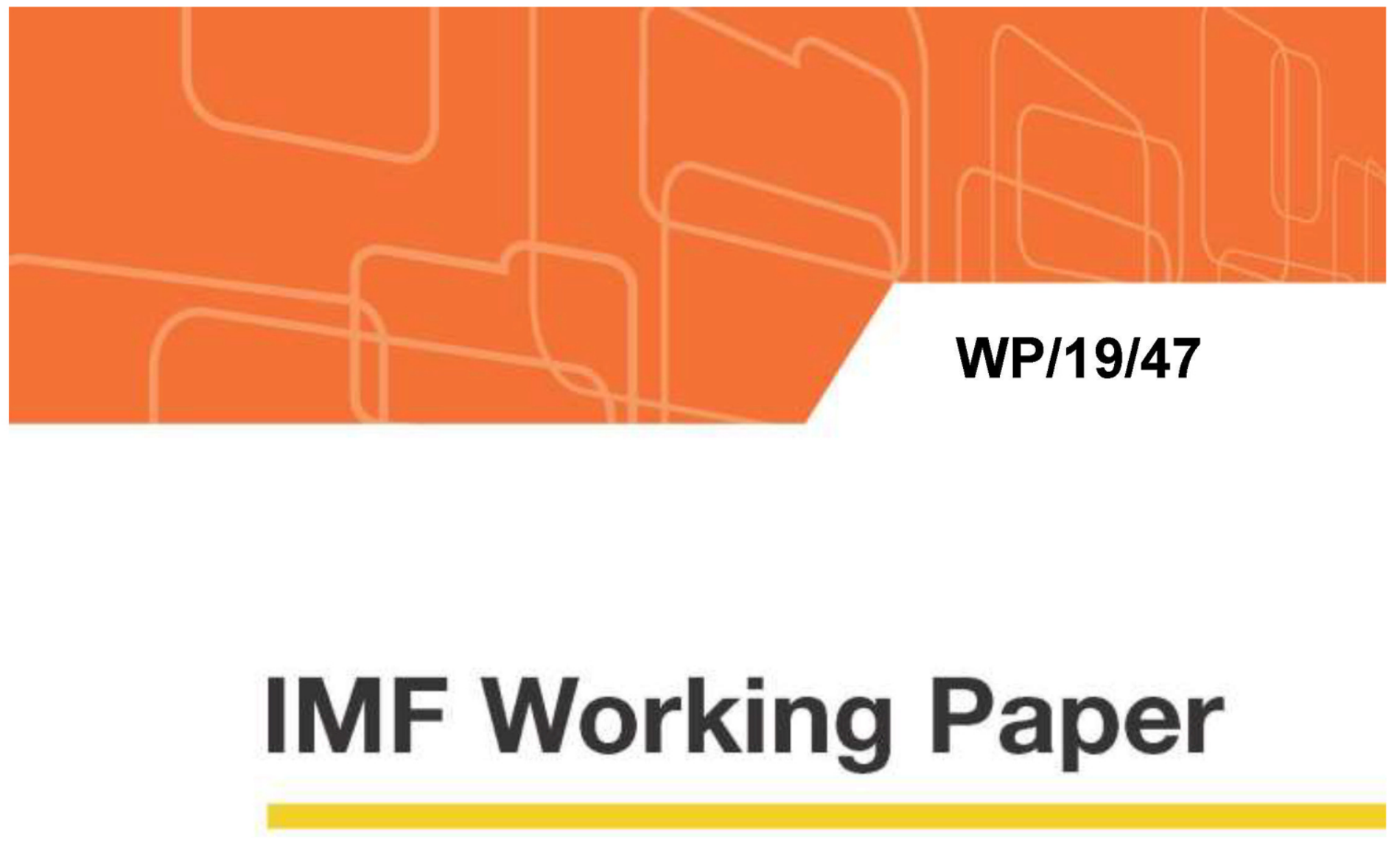

\title{
Struggling to Make the Grade: A Review of the Causes and Consequences of the Weak Outcomes of South Africa's Education System
}

by Montfort Mlachila and TIhalefang Moeletsi

IMF Working Papers describe research in progress by the authors and are published to elicit comments and to encourage debate. The views expressed in IMF Working Papers are those of the authors and do not necessarily represent the views of the IMF, its Executive Board, or IMF management. 


\section{WP/19/47}

\section{IMF Working Paper}

\section{Struggling to Make the Grade: A Review of the Causes and Consequences of the Weak Outcomes of South Africa's Education System}

by Montfort Mlachila and TIhalefang Moeletsi

IMF Working Papers describe research in progress by the authors and are published to elicit comments and to encourage debate.

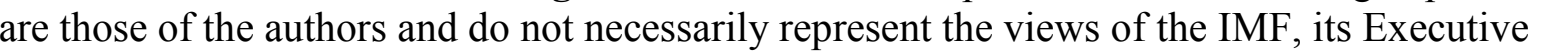

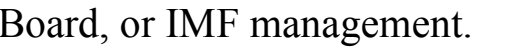

I NTERNATIONAL MONETARY FUND 


\title{
IMF Working Paper
}

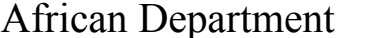 \\ Struggling to Make the Grade: \\ A Review of the Causes and Consequences of the Weak Outcomes \\ of South Africa's Education System
}

\section{Prepared by Montfort Mlachila and Tlhalefang Moeletsi}

$\square|\|\| \| \square| \square$

\begin{abstract}
IMF Working Papers describe research in progress by the authors and are published to

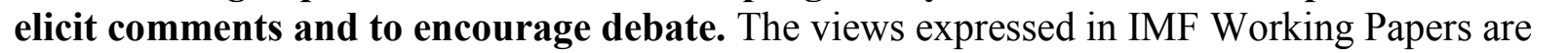

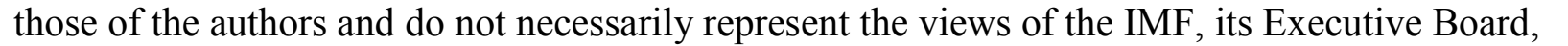

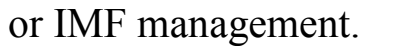

\begin{abstract}

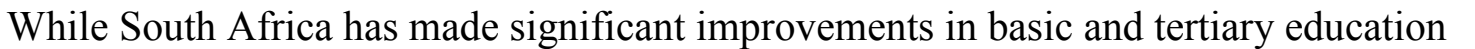

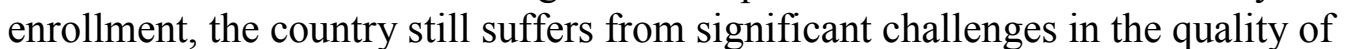

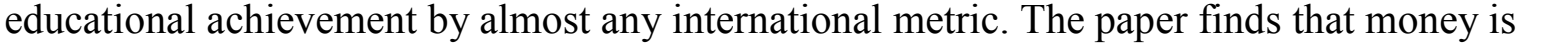
clearly not the main issue since the South Africa's education budget is comparable to

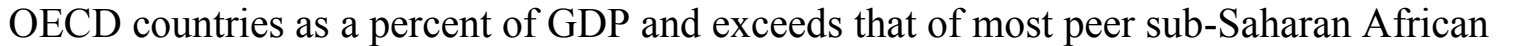

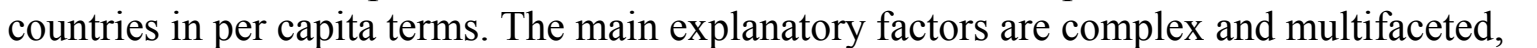

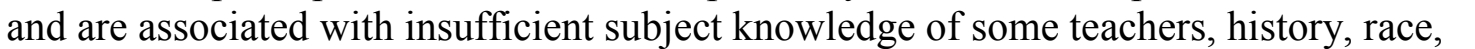

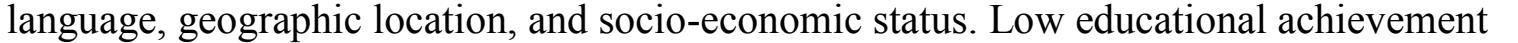

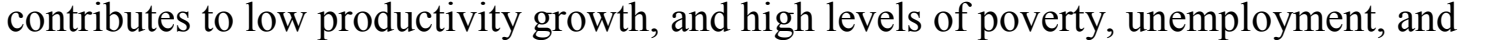
ए

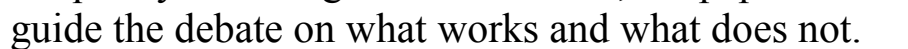

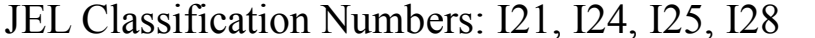

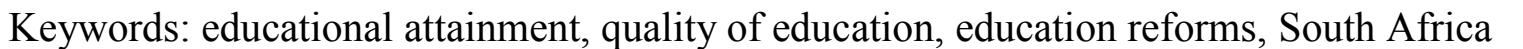

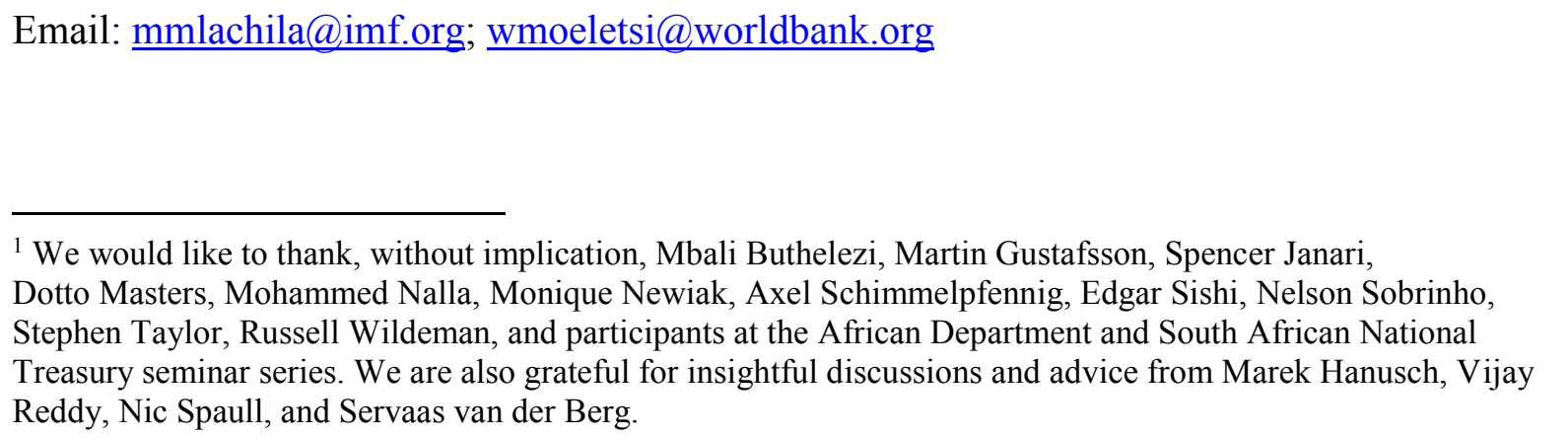

CInternational Monetary Fund. Not for Redistribution 


\section{Contents}

$\square$

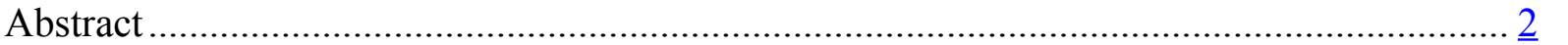

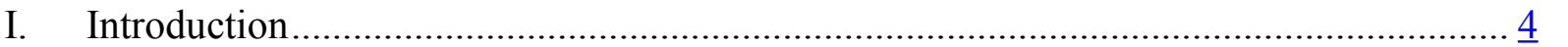

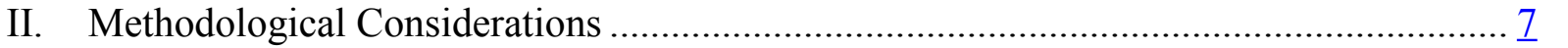

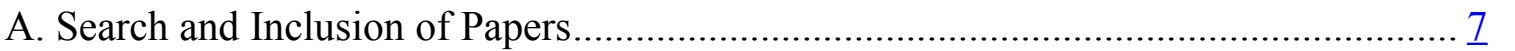

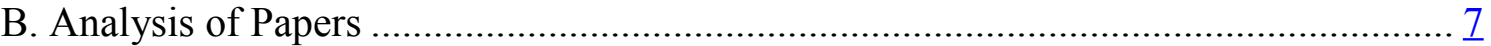

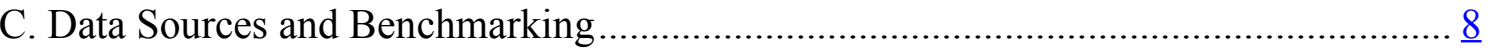

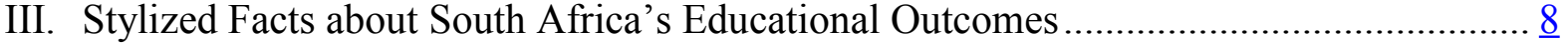

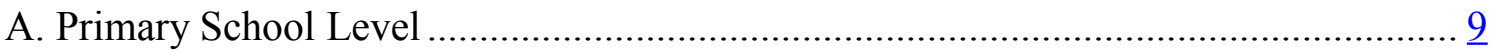

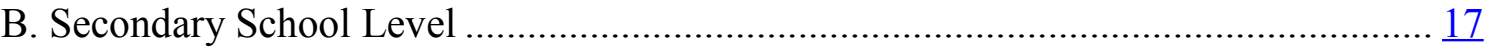

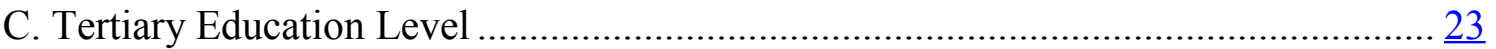

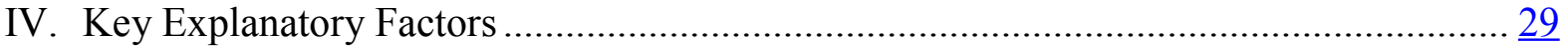

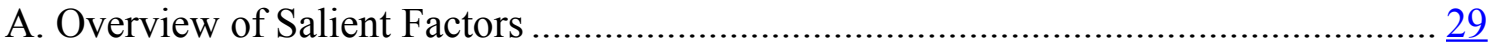

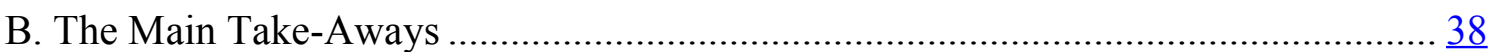

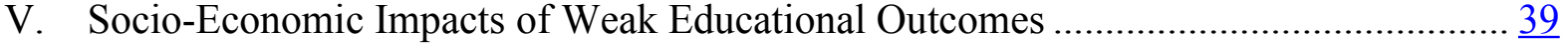

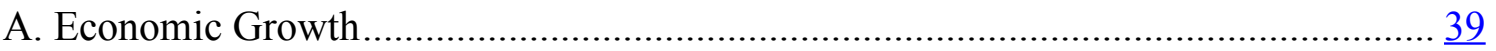

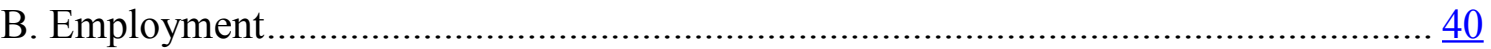

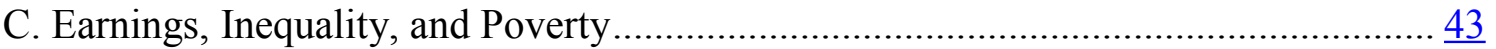

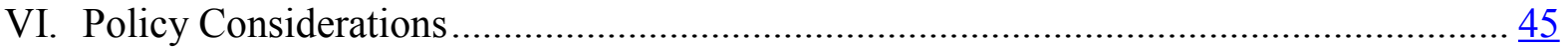

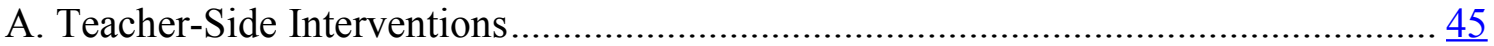

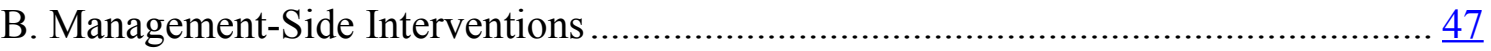

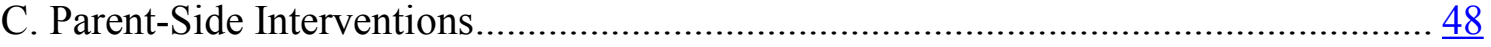

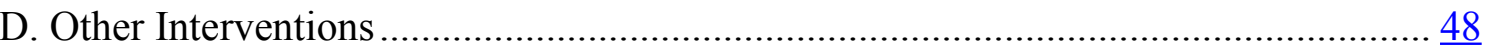

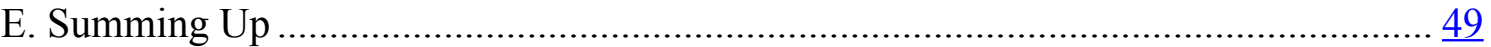

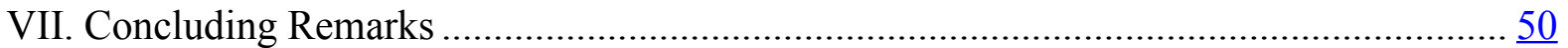

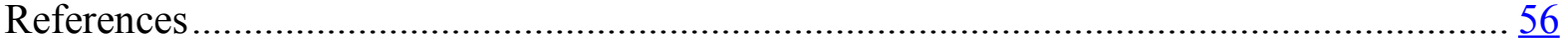
$\square$

$\square$ 


\section{INTRODUCTION}

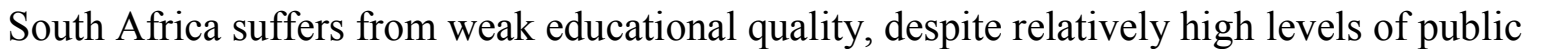

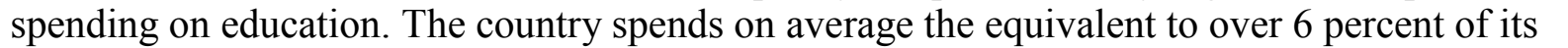

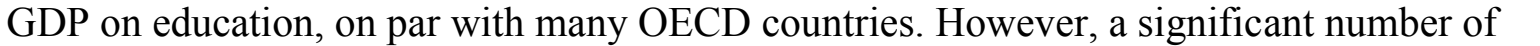

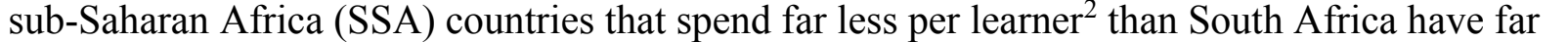

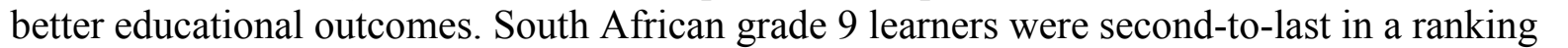

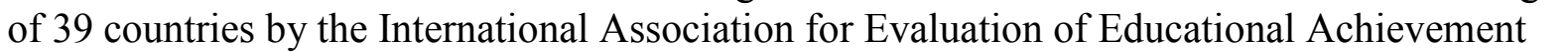

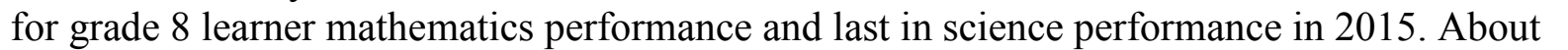
half of South Africa's students drop out of school before completing secondary education.

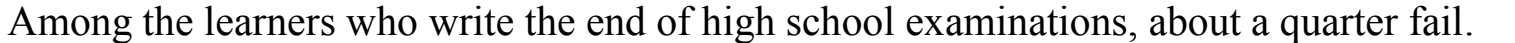

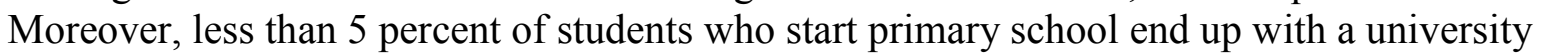
$\square \square\|\|\|\|\|\| \| \square$

$\square$

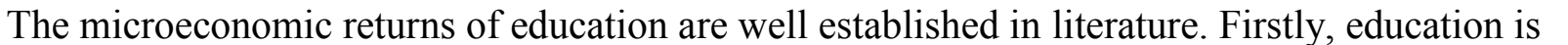

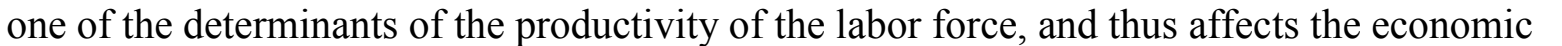

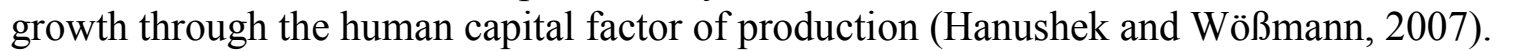

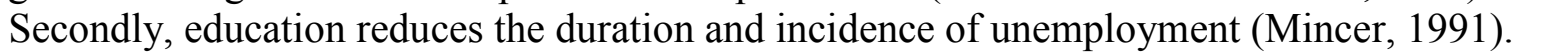

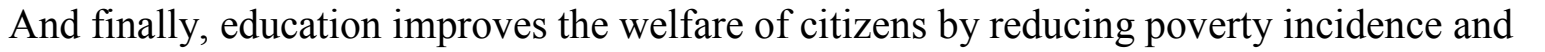

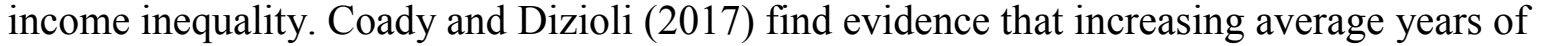

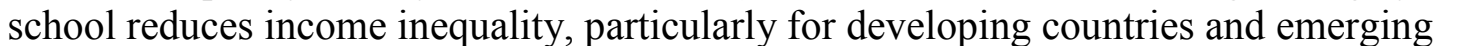

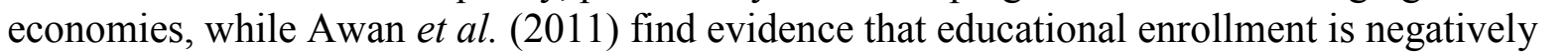

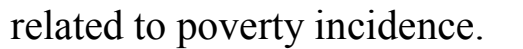

$\square$

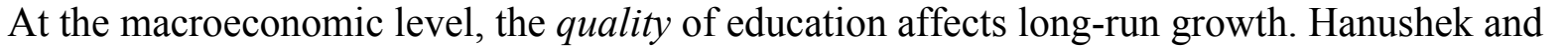

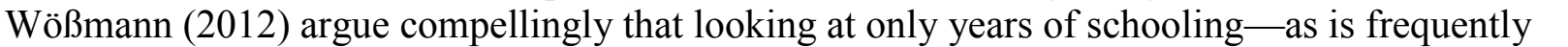

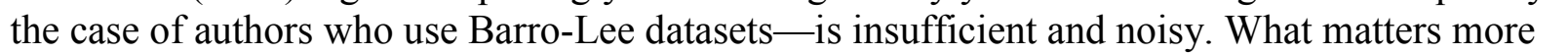

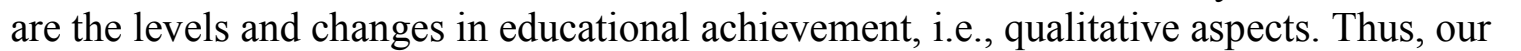

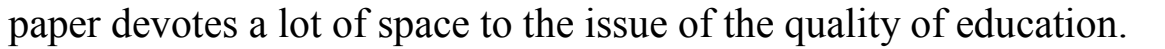

$\square$

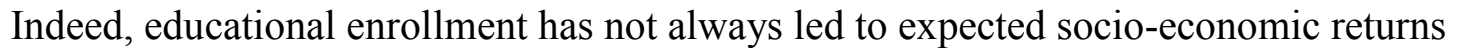

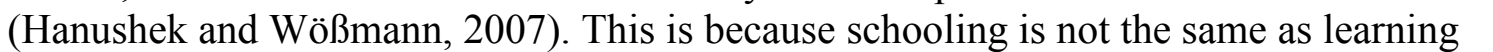

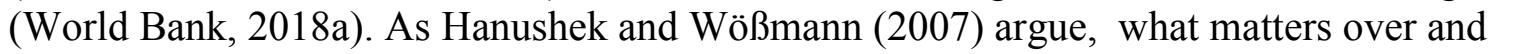

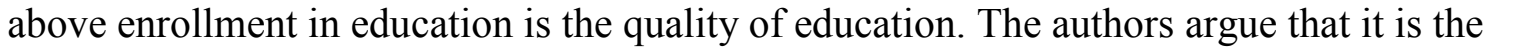

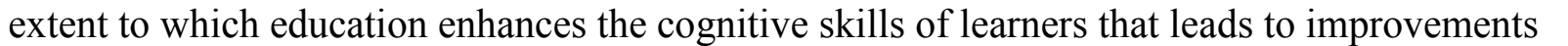

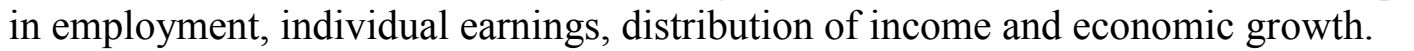

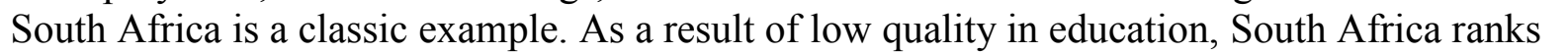

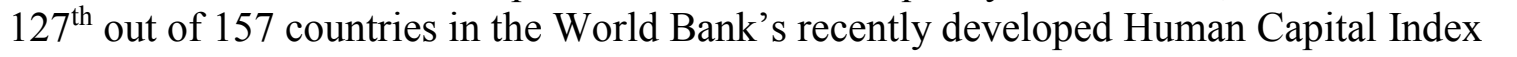

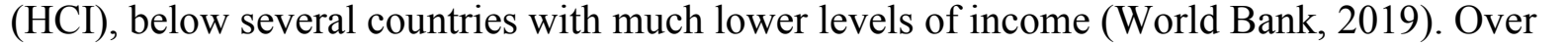
ए

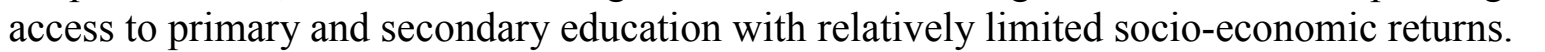

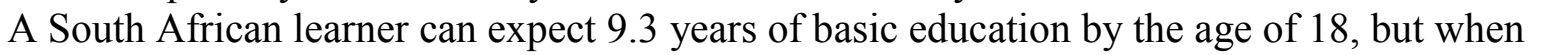

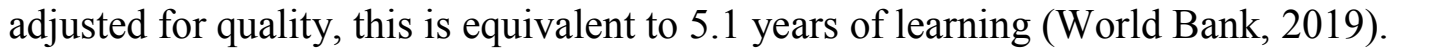
$\square$

mIm!m

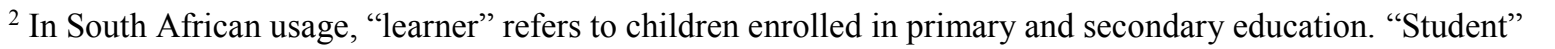

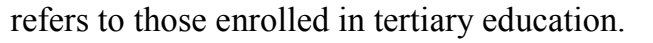




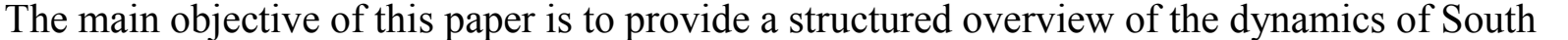

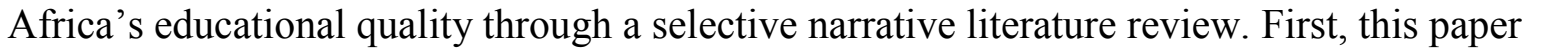
brings out the key stylized facts about South Africa's education system, focusing on inputs

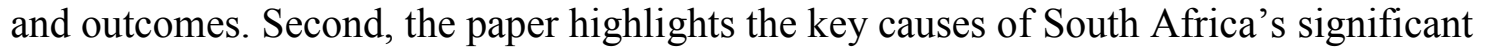

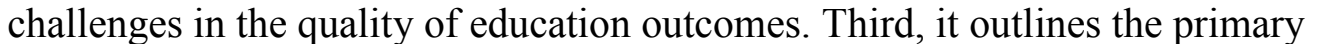

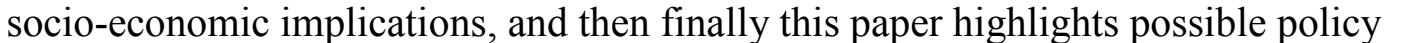

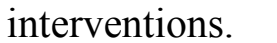

$\square$

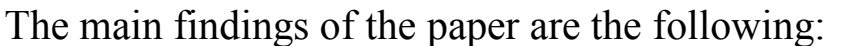

$\square$

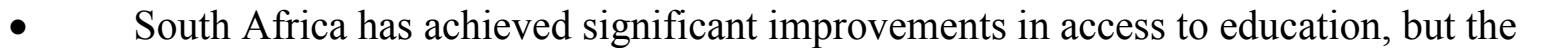

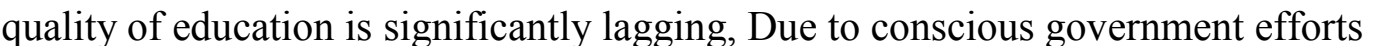

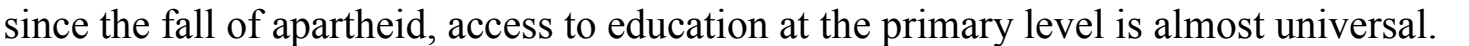

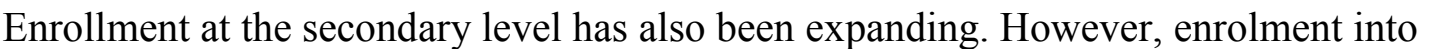

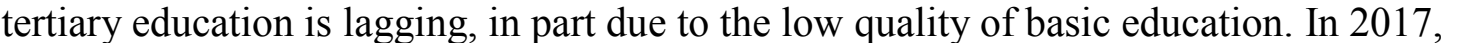

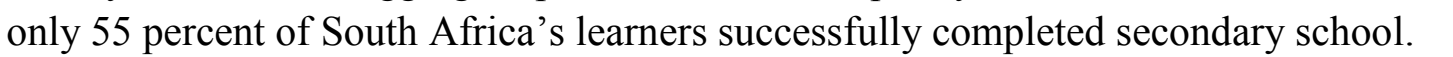

$\square$

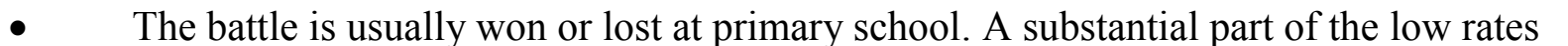

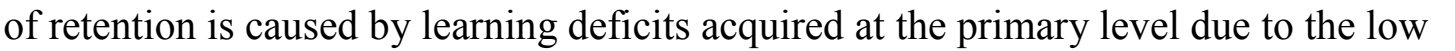

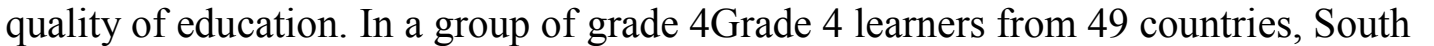

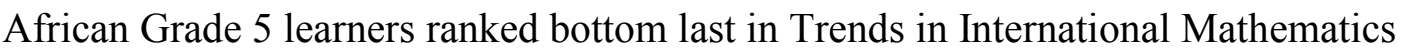

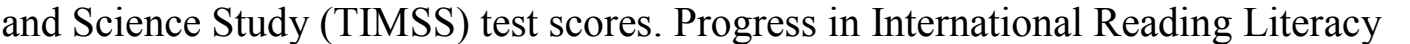

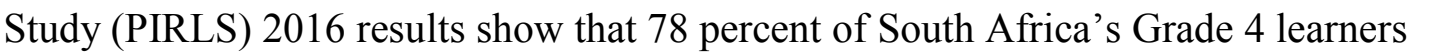

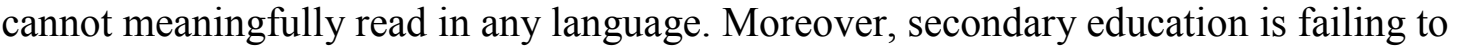

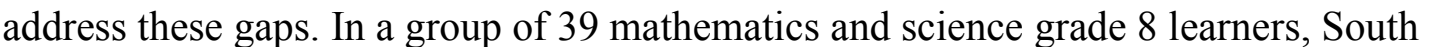

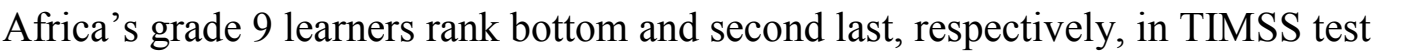

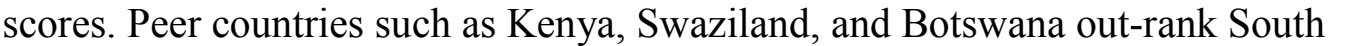

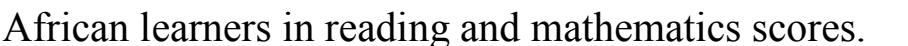

$\square$

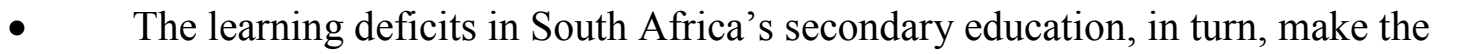

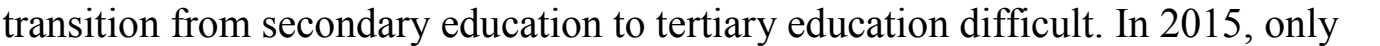

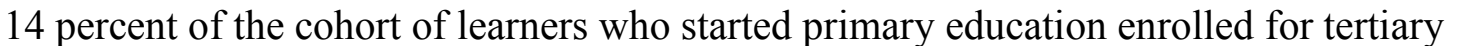

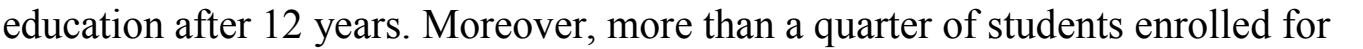

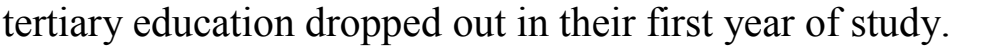

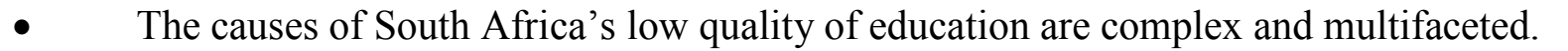

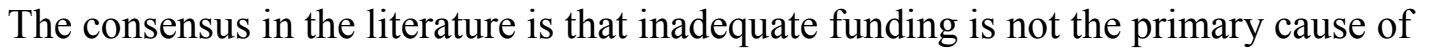

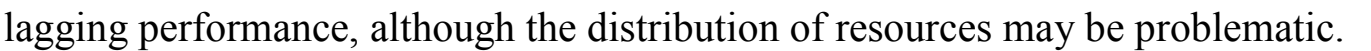

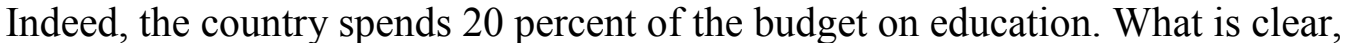

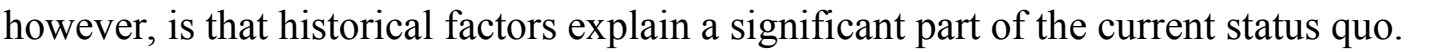

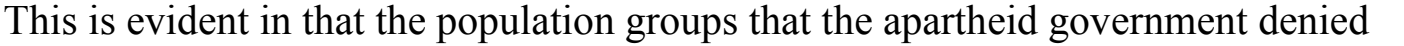

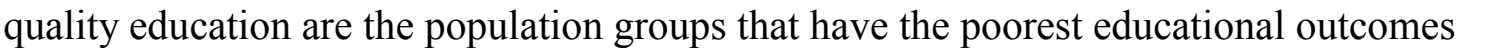
today. At a primary and secondary level, South Africa's education sy $\square \square \square \square \square \square \square \square \square \square$

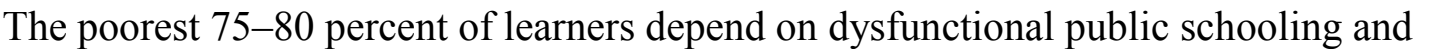




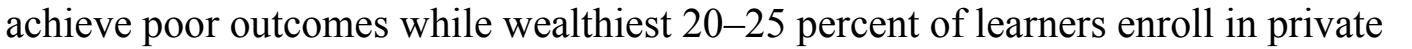

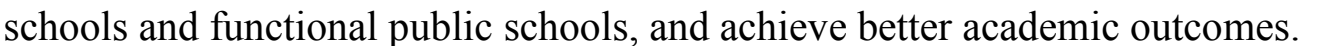

$\square$

- $\quad$ The bimodality of South Africa's education system is perpetuating economic

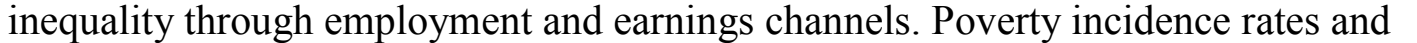

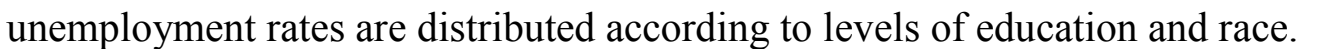

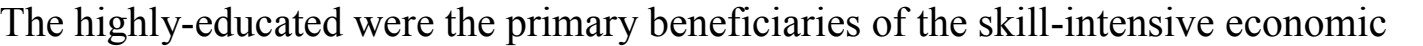

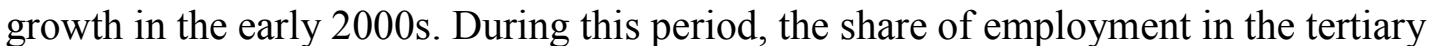

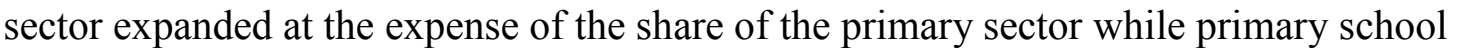

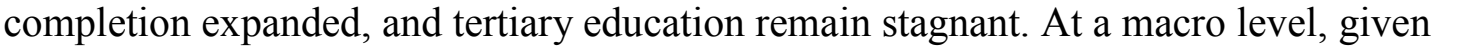

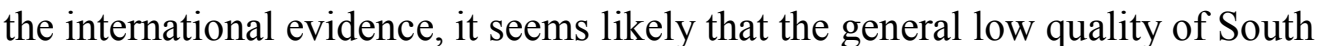

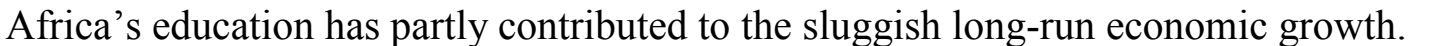

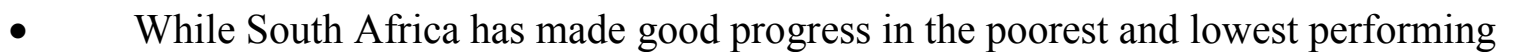

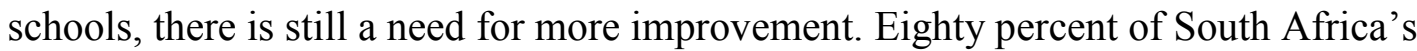

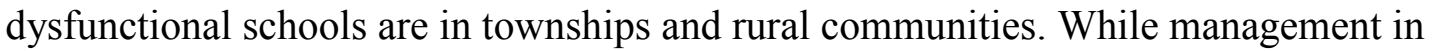
ए।

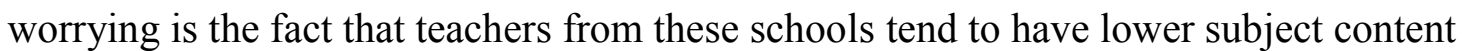

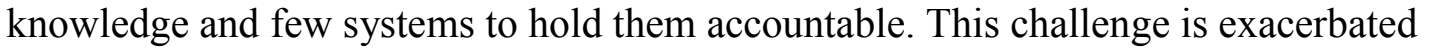

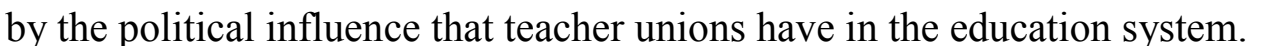

$\square$

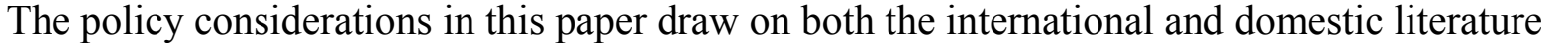

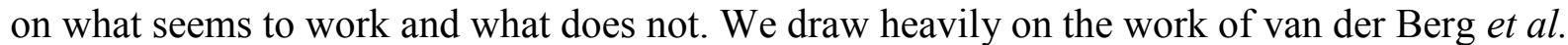

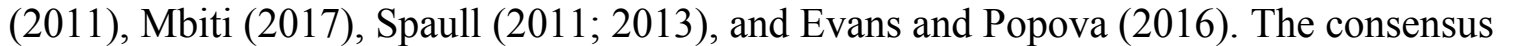

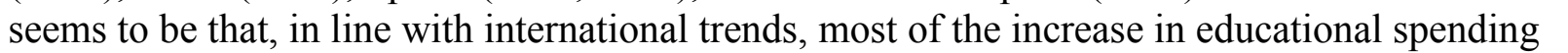

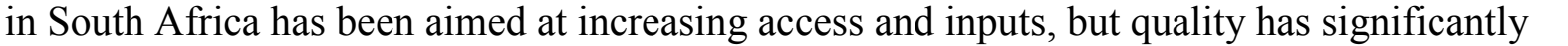

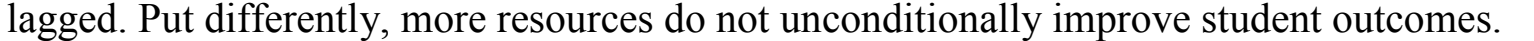

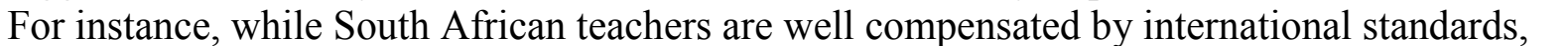

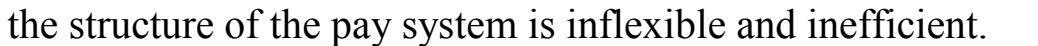

$\square$

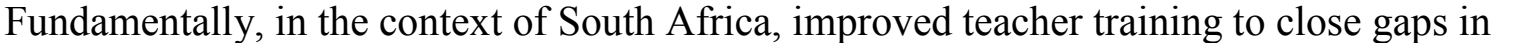

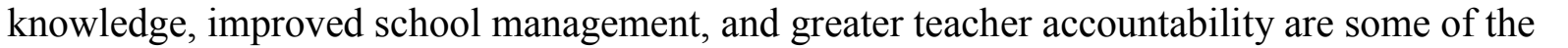

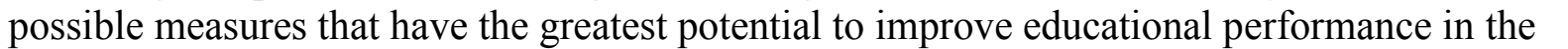

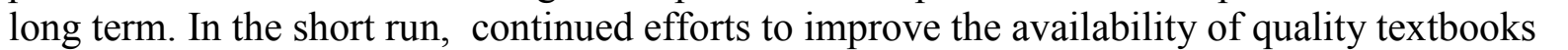

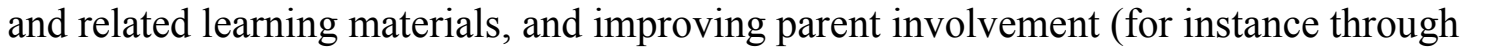

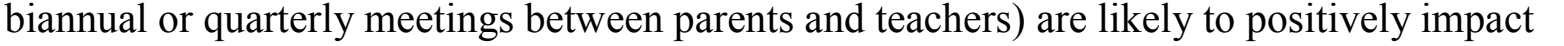

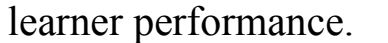

$\square$

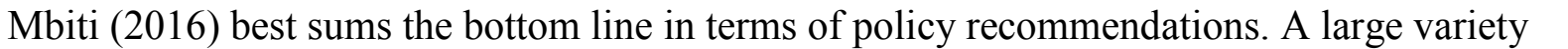

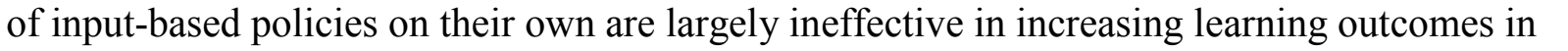

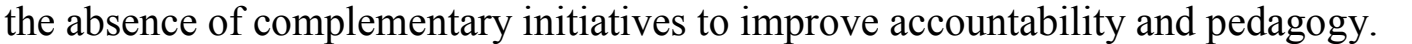

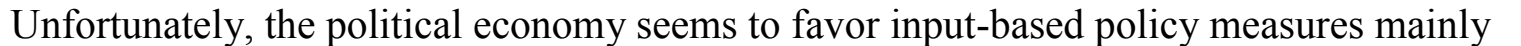

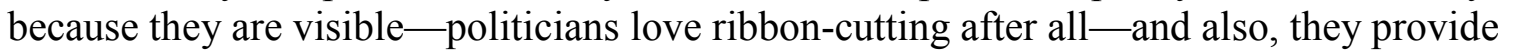

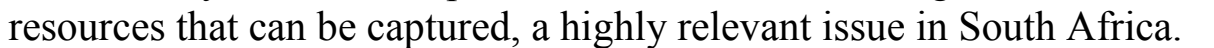
$\square$ 


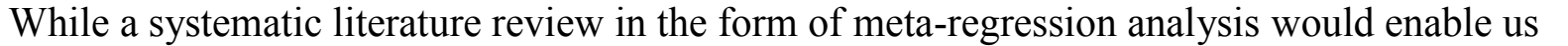

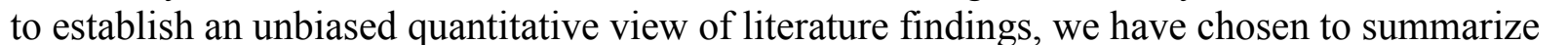

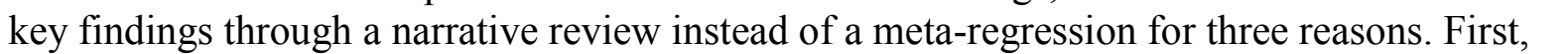

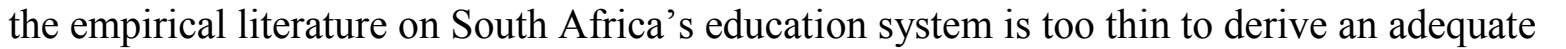

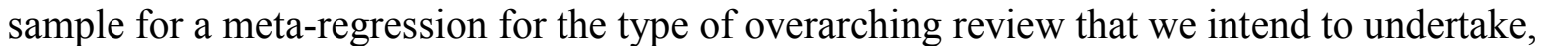

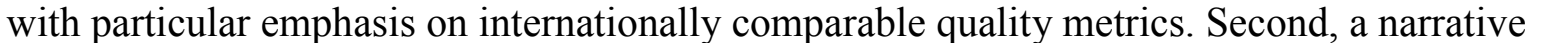

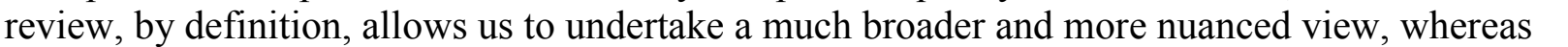

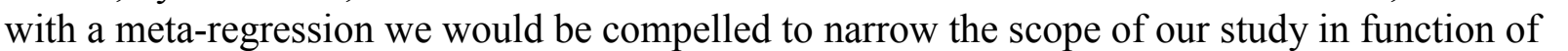

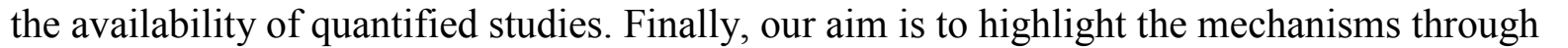

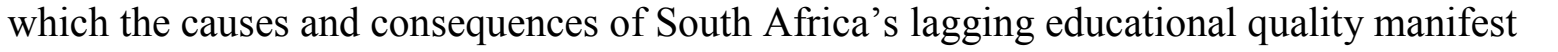

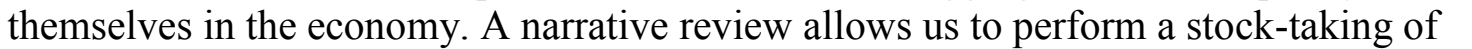

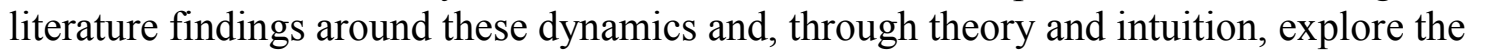

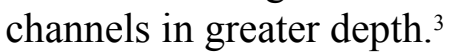

$\square$

\section{Methodological Considerations}

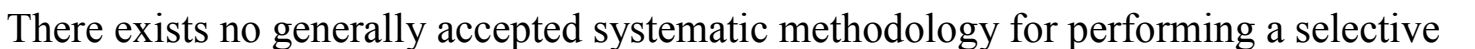

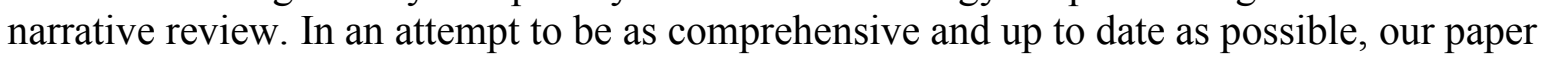

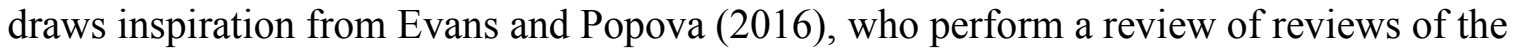
प I

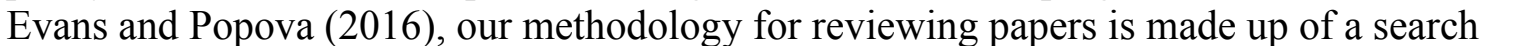

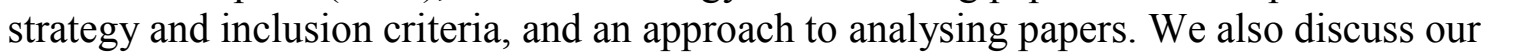

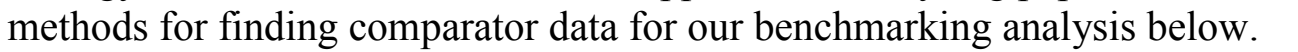

\section{A. Search and Inclusion of Papers $\square$}

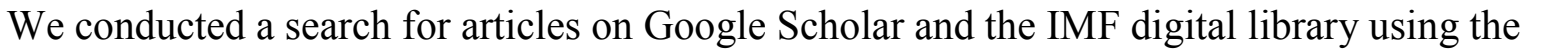
$\square \square\|\|\|\|$ "education in South Africa" and "education in developing countries" $\square \square \square\|\| \| \square \square$

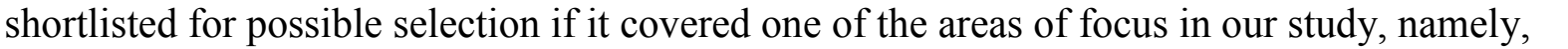

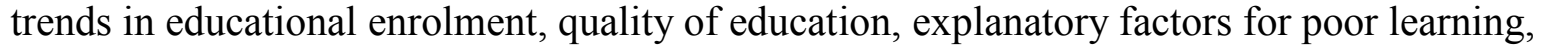

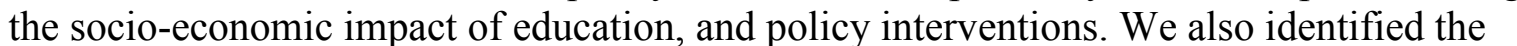

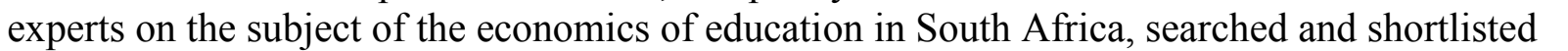

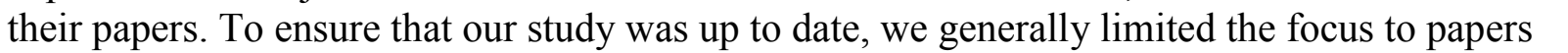

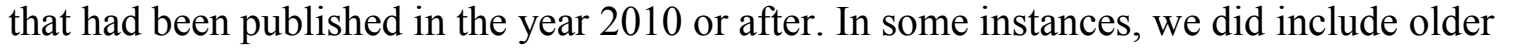

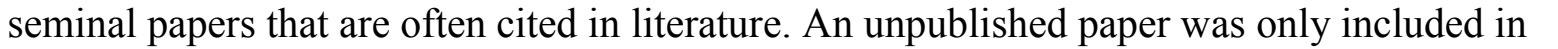

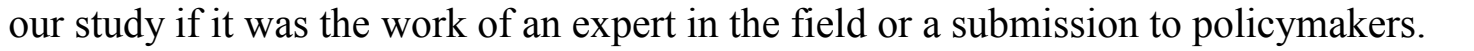

\section{B. Analysis of Papers $\square$}

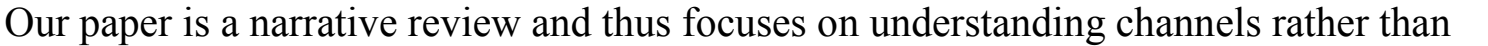

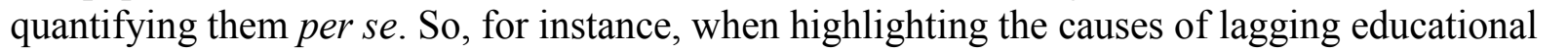

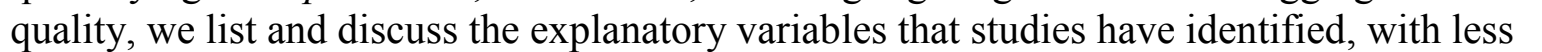

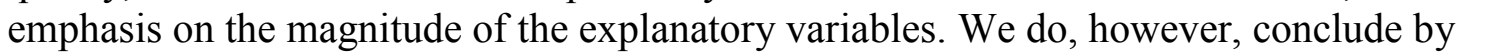

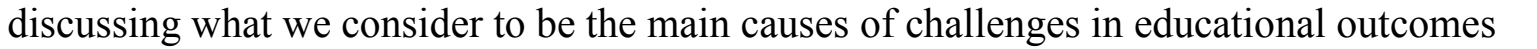

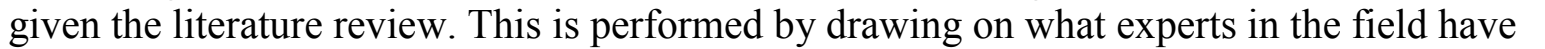

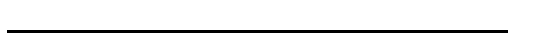

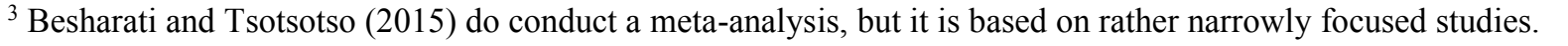




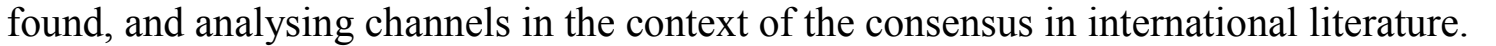

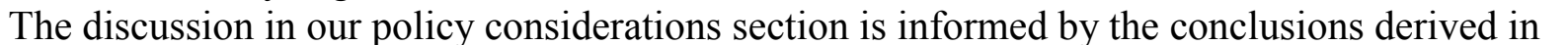

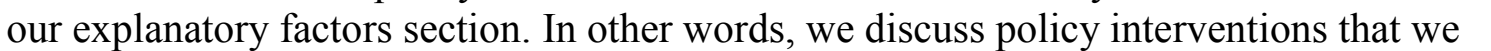

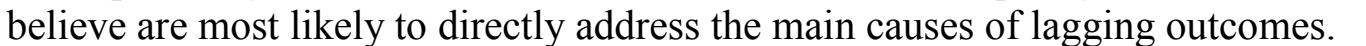
$\square$

\section{Data Sources and Benchmarking $\square$}

$\square$ order to establish stylized facts about South Africa's education system and $\square \square \square \square \square$

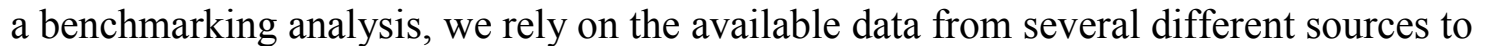

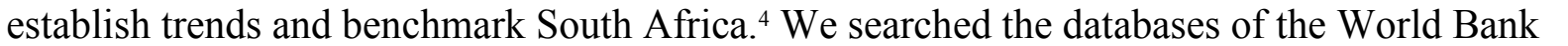

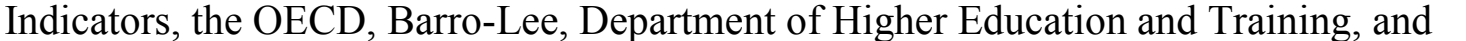

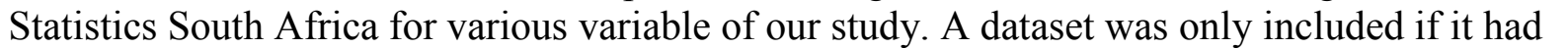

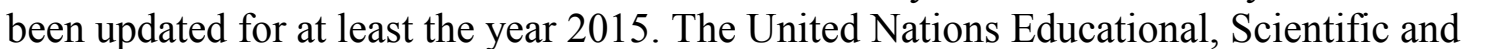

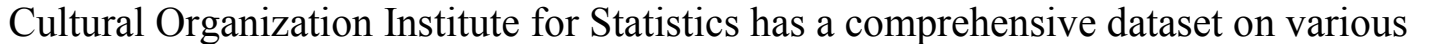

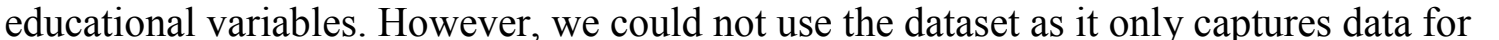

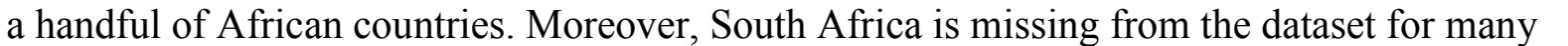

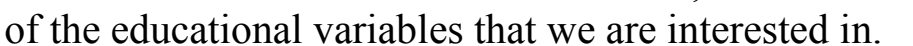

$\square$

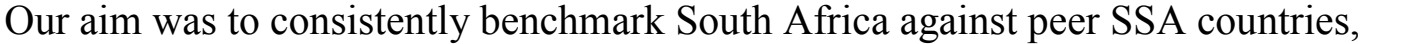

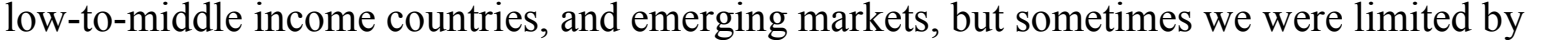

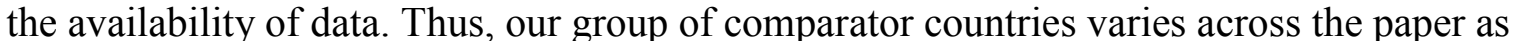

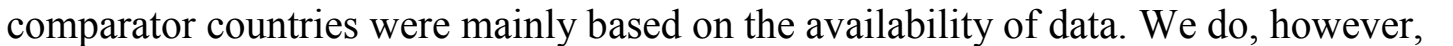

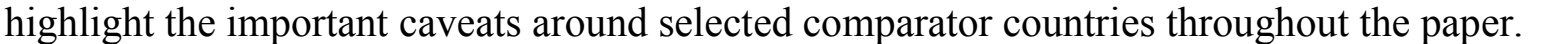

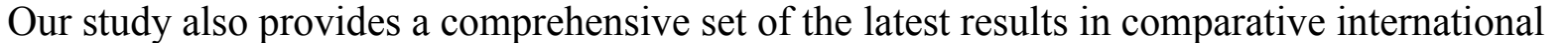

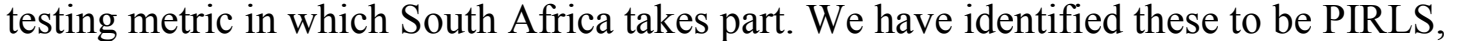

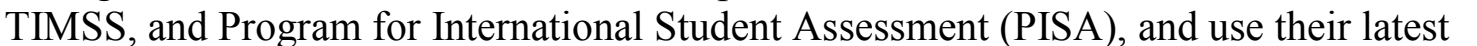

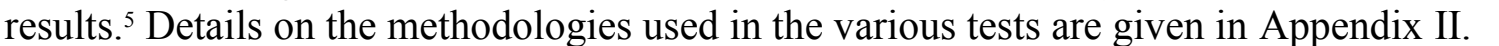

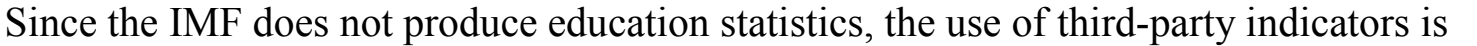

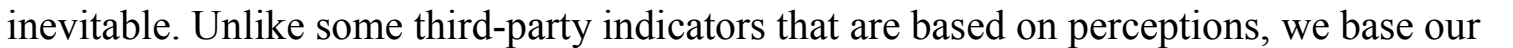

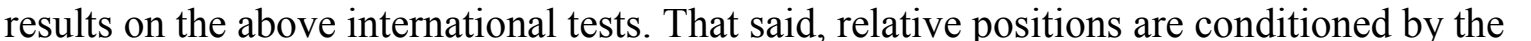

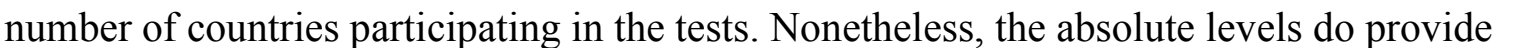

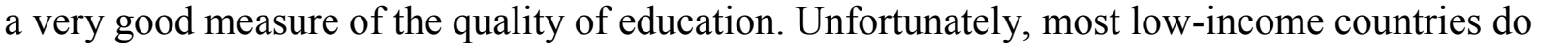

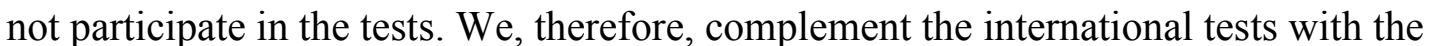

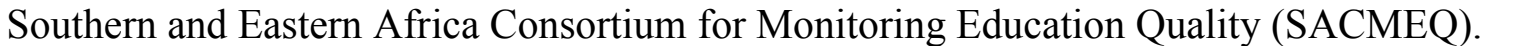
$\square$

\section{Stylized Facts about South Africa’s Educational OUTCOMES}

This section presents an overview of the key stylized facts in South Africa's education system

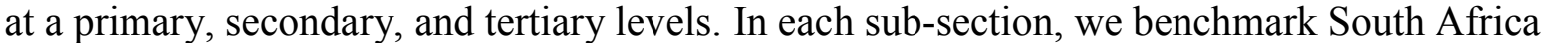

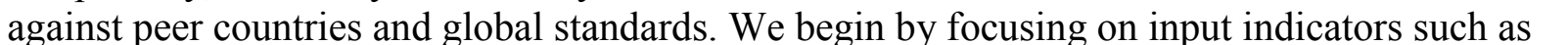

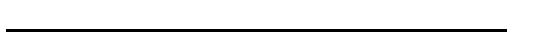

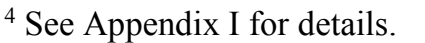

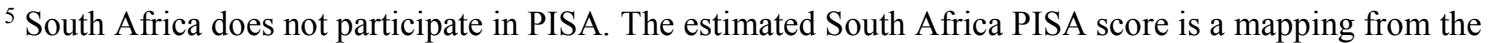

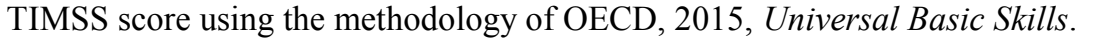




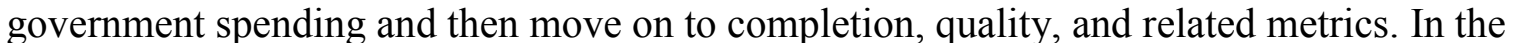

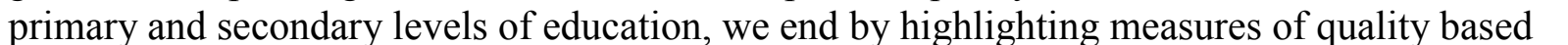

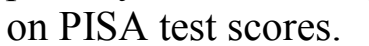

$\square$

\section{A. Primary School Level}

\section{Input indicators}

South Africa's expenditure on education has been increasing in recent decades and the level of

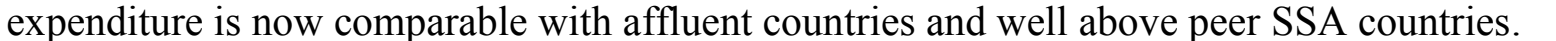

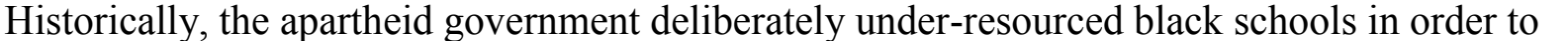

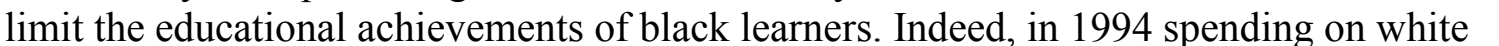

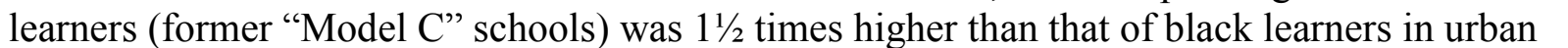

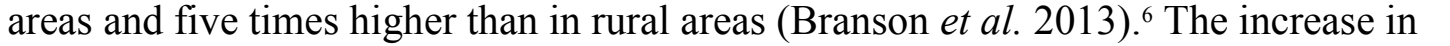

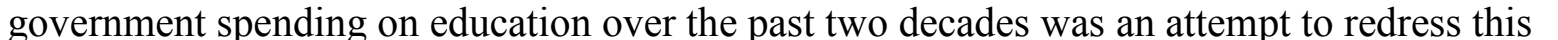

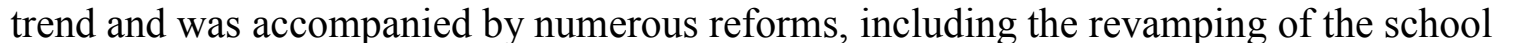

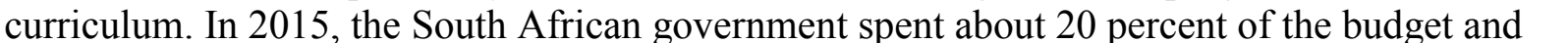

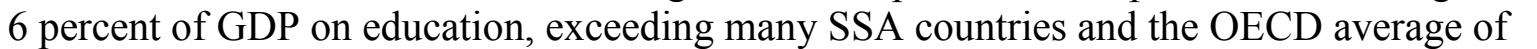

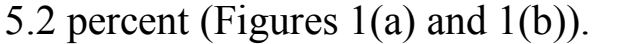

$\square$

Figure 1 (a). Expenditure on Education as a Proportion of Total Government Expenditure in 2015

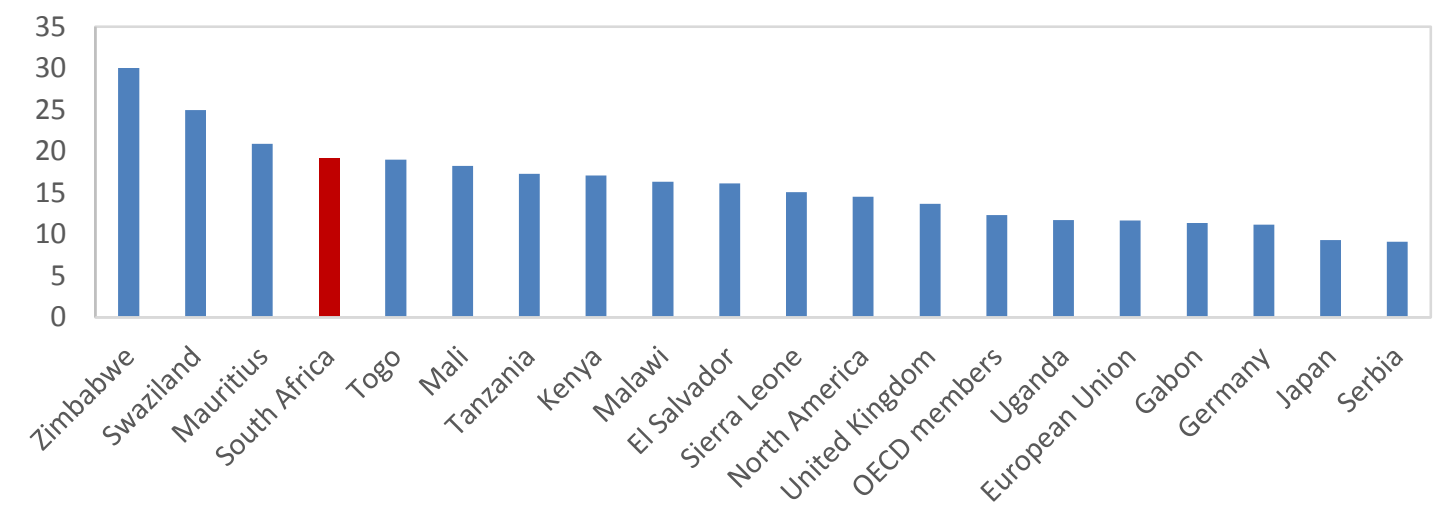

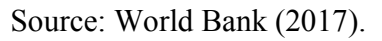

$\square$

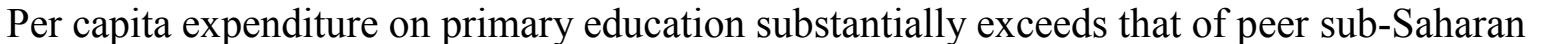

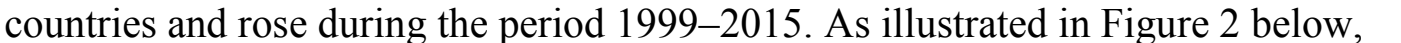
पDIUAfrica's spending on primary education as a percentage of GDP per capita increased

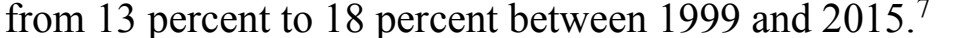

$\square$

m

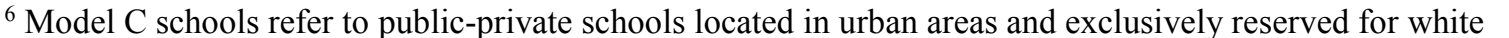

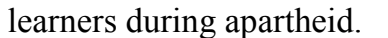

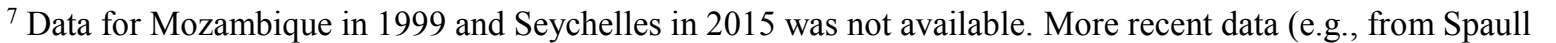
एमा

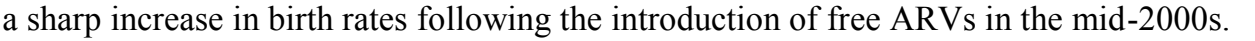


Figure 1 (b). Government Expenditure on Education as a Proportion of GDP in 2015

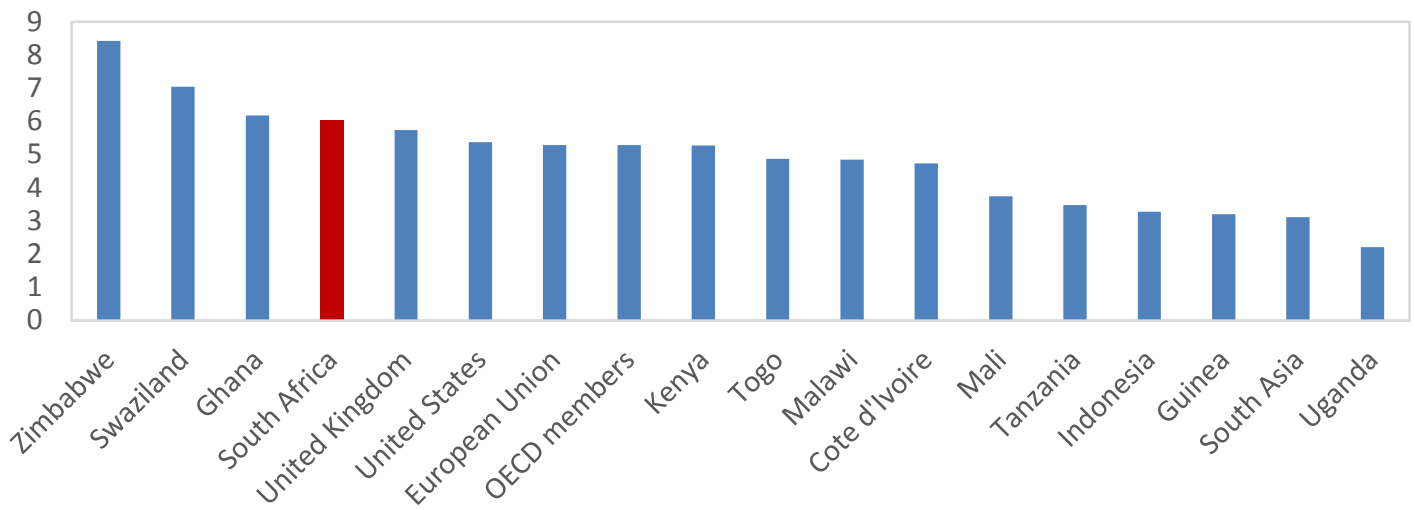

Figure 2. Government Spending on Primary Education per Student as a Percentage of GDP per Capita

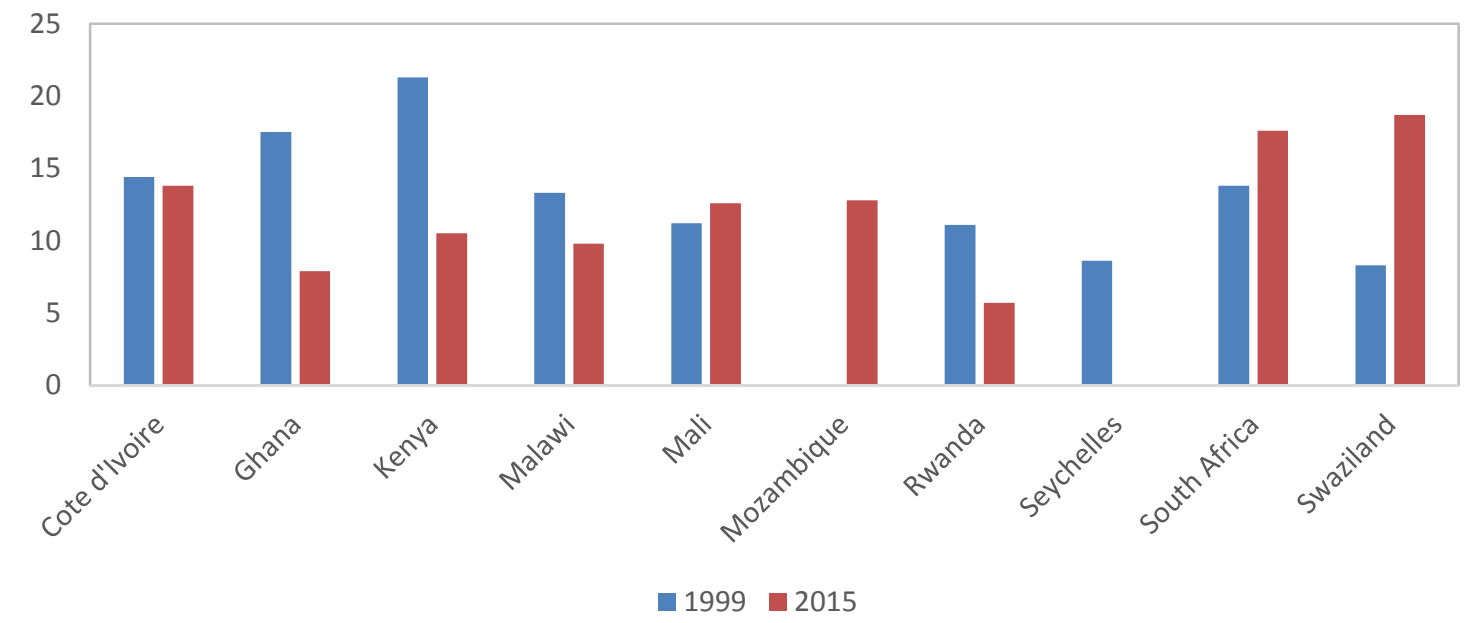

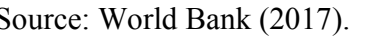

\section{Enrollment indicators}

$\square$

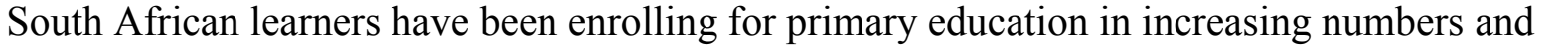

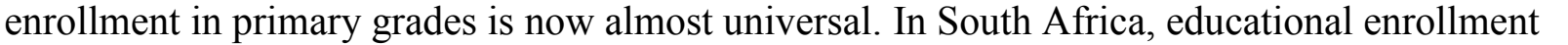

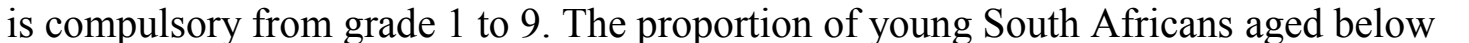

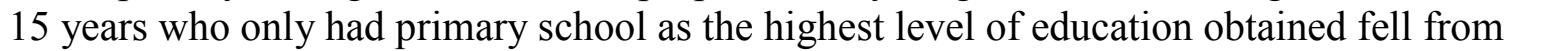

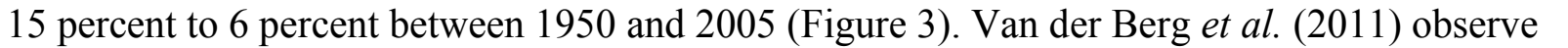

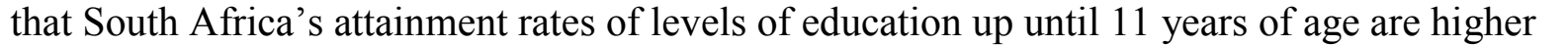

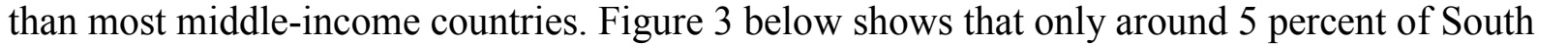

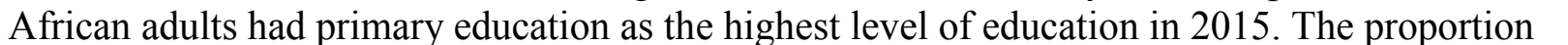

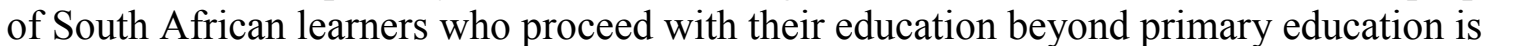




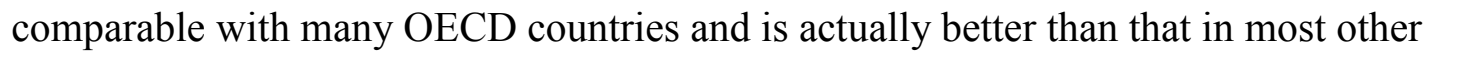

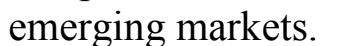

$\square$

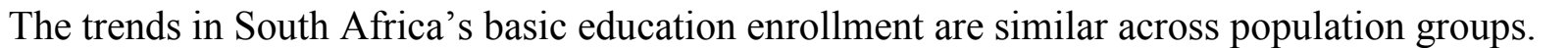

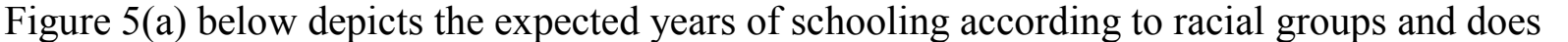

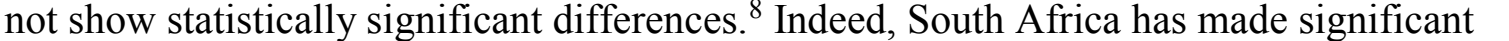

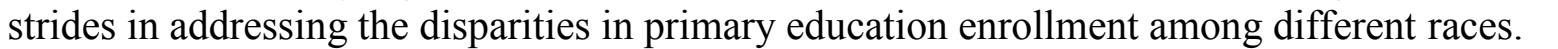

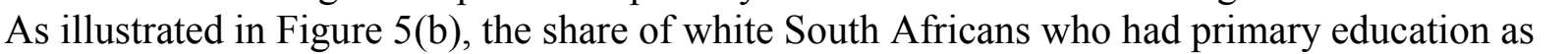

Figure 3. Proportion of Population over 15 Years Who Had Primary Education as their Highest Level of Education

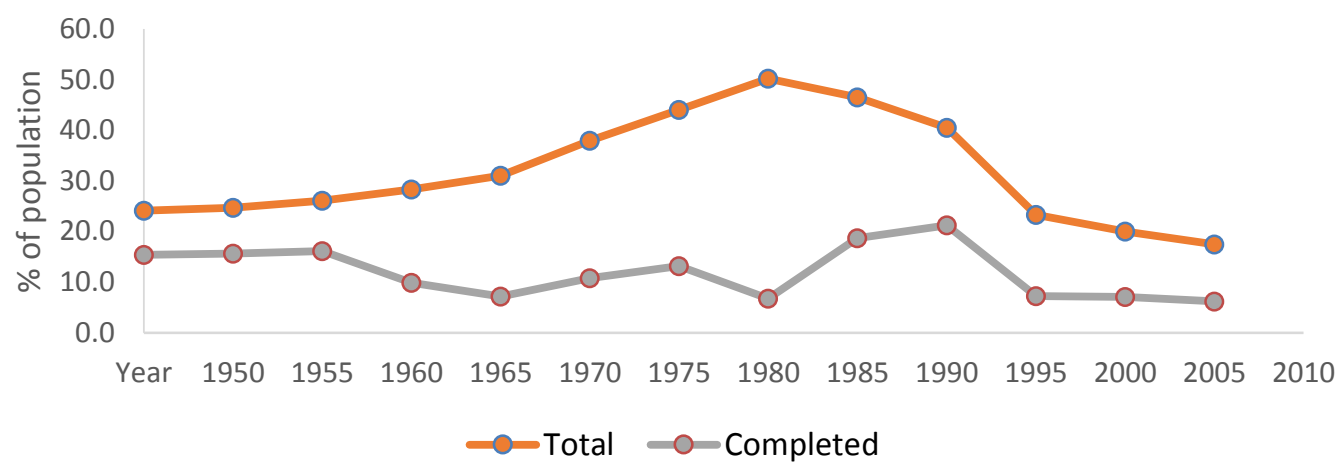

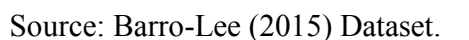

Figure 4. Proportion of Population Aged between 25 and 64 Whose Highest Level of Education Was at a Primary Level in 2015

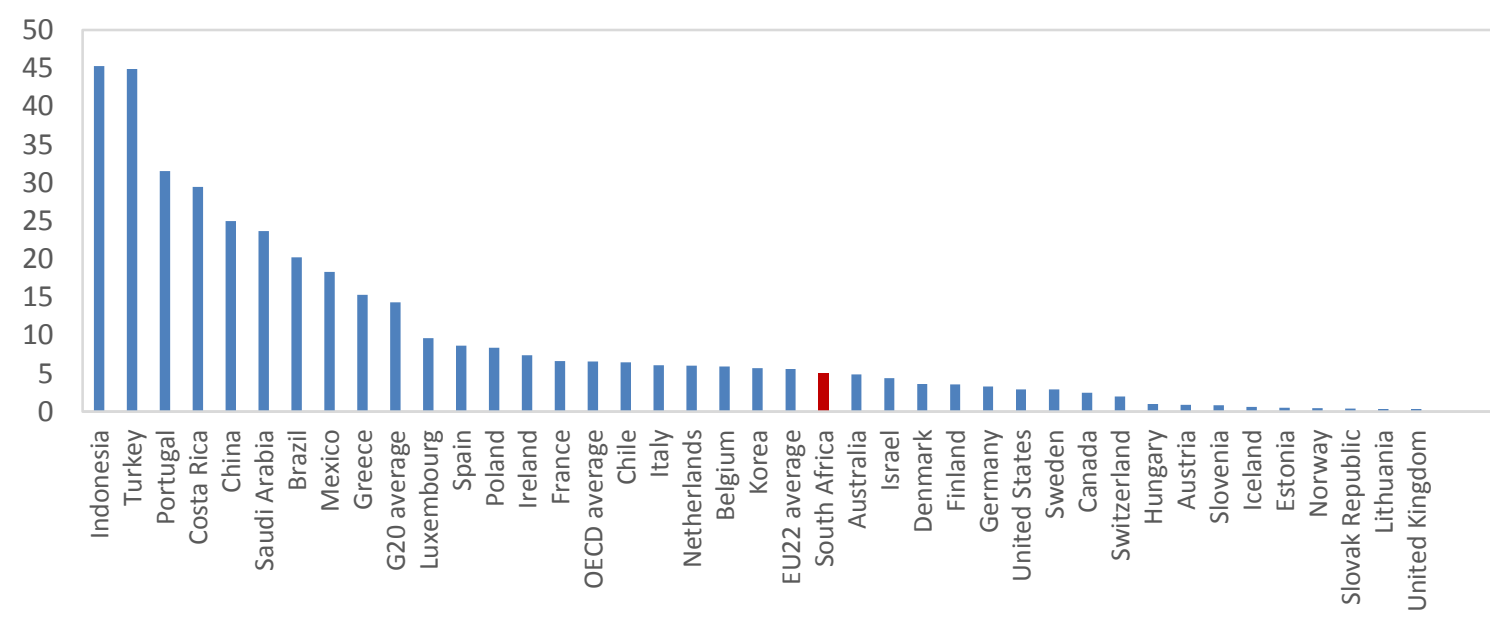

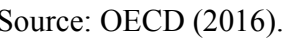

पा

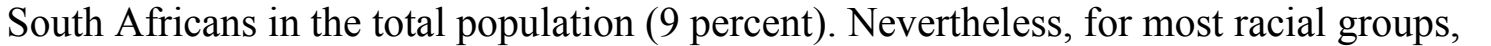

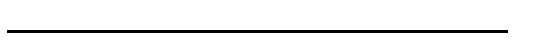

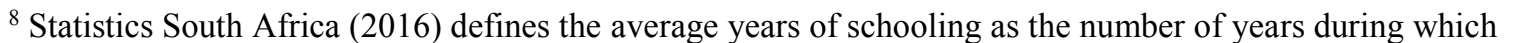

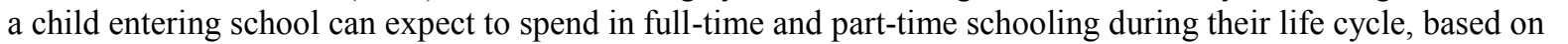

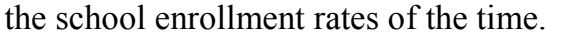


Figure 5 (a). South Africa's Average Years of Primary Schooling by Population Group in 2016

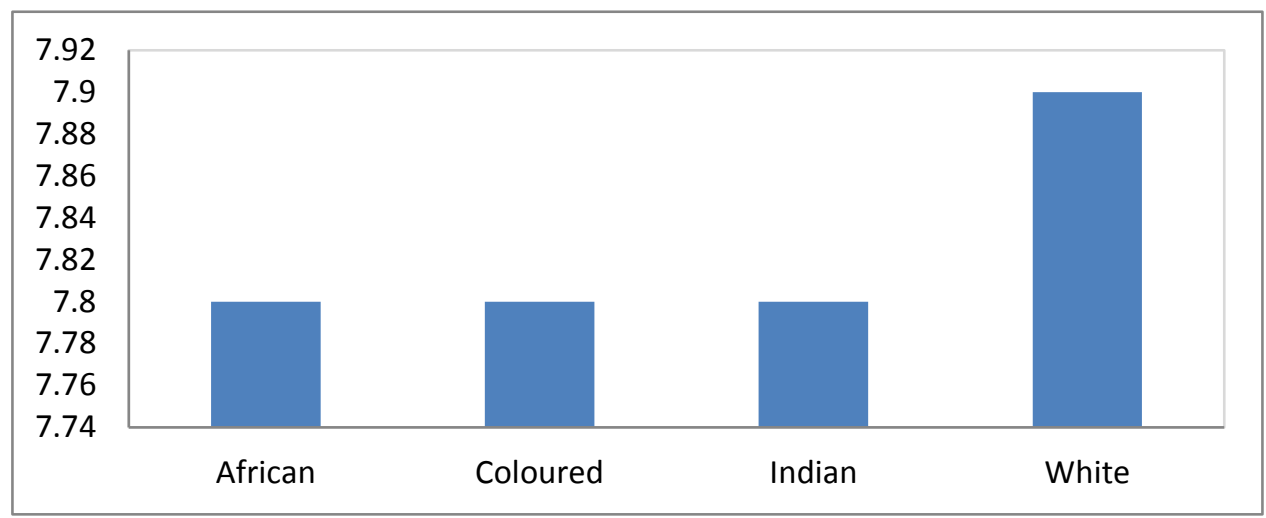

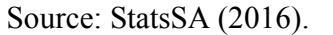

Figure 5 (b). South Africa's Proportion of Individuals Aged 25-64 Whose Highest Level of Education was Primary Education in 2016

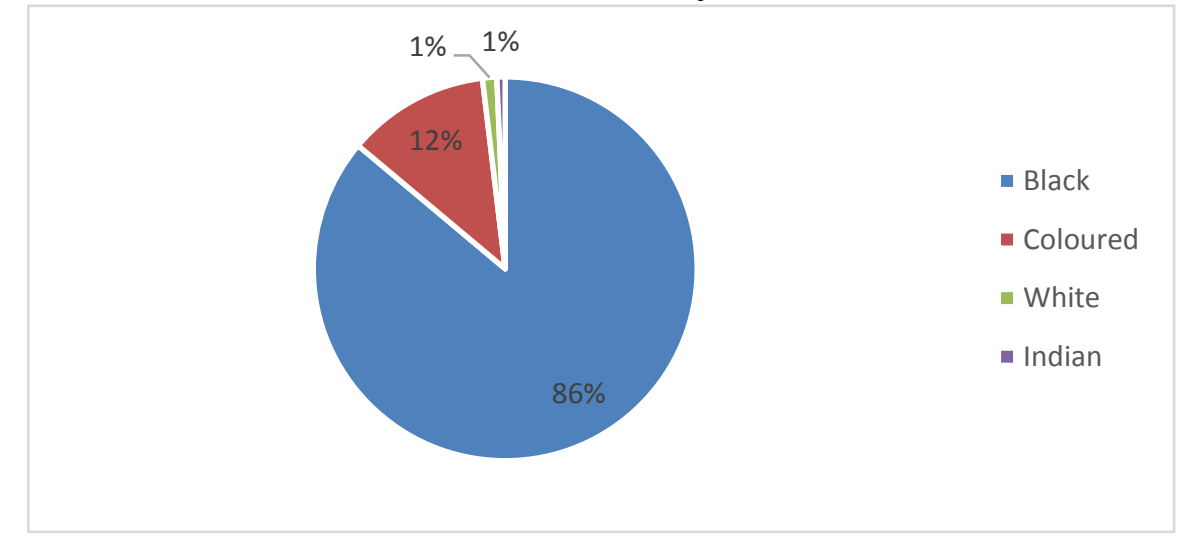

\section{Quality indicators}

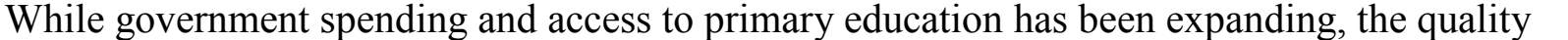

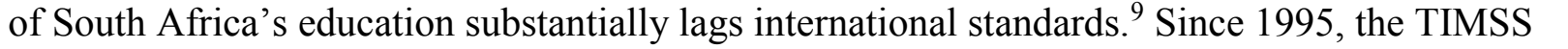

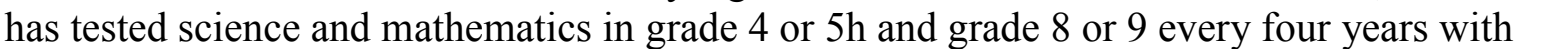

\footnotetext{
mmmmmmmmmmmmm

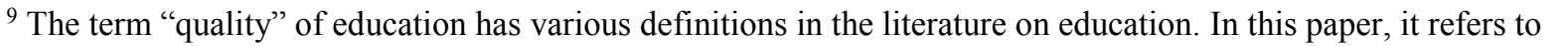

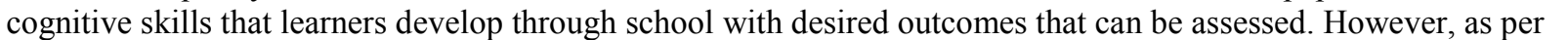

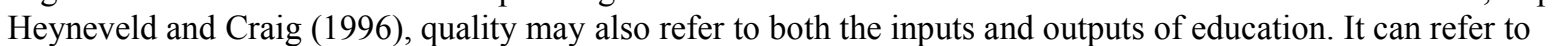

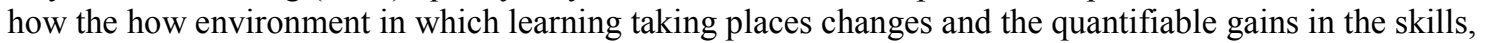

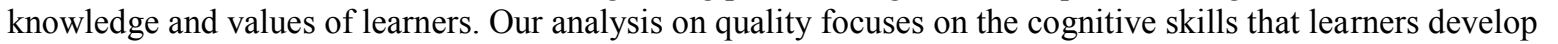

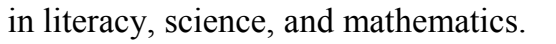

CInternational Monetary Fund. Not for Redistribution 


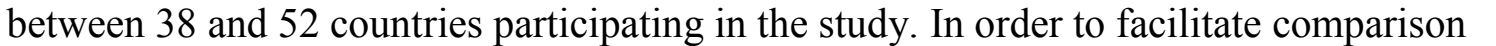

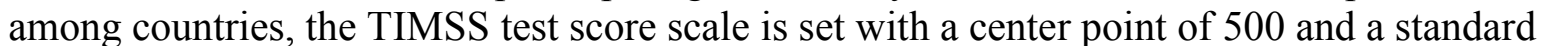

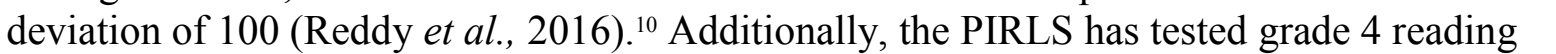

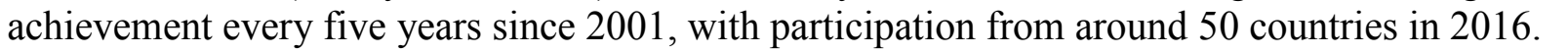

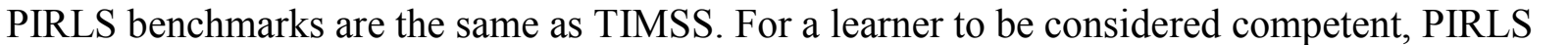

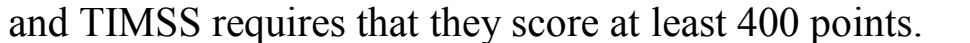

$\square$

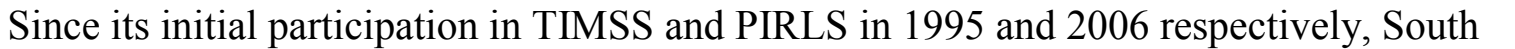

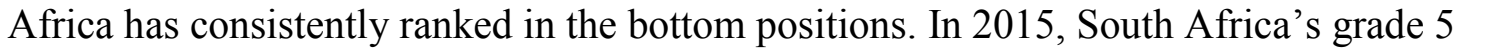

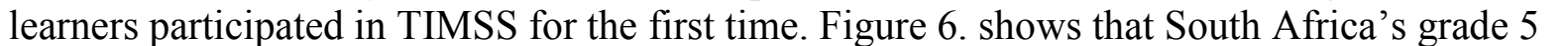

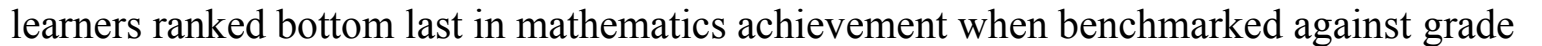

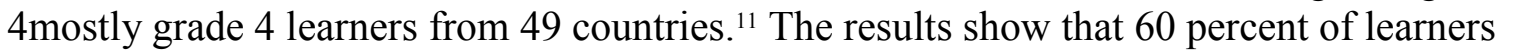

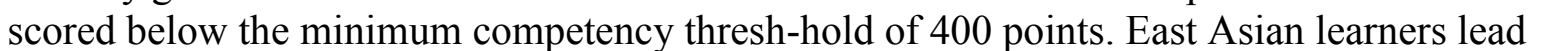

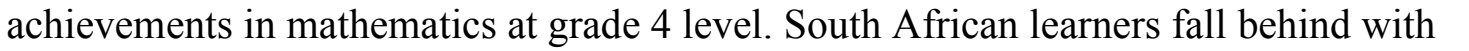

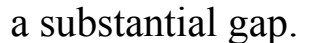

$\square$

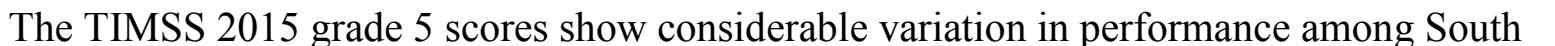

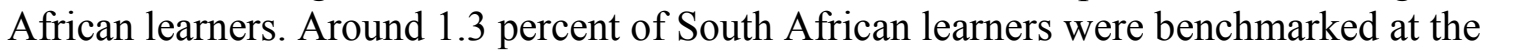

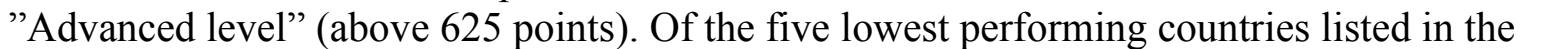

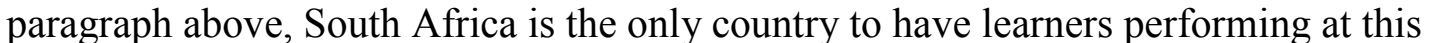
“a

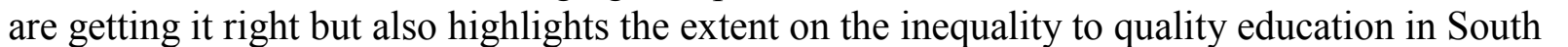

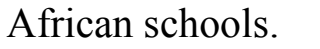

$\square$

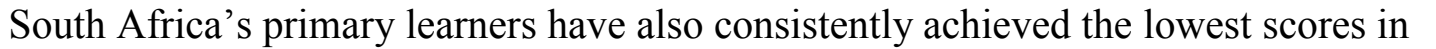

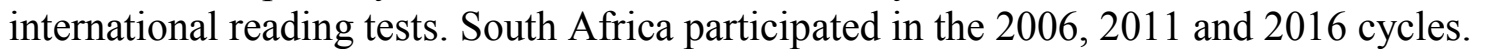

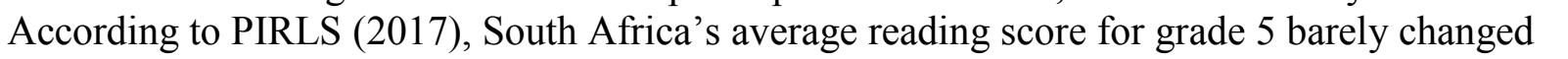

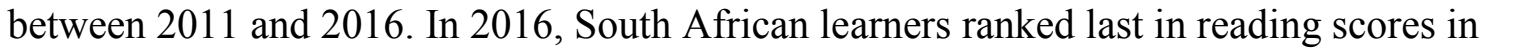

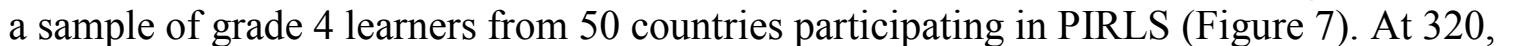

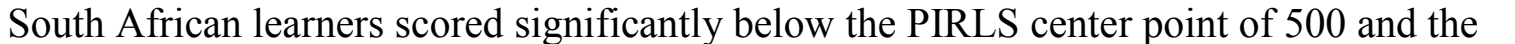

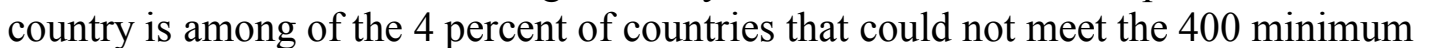

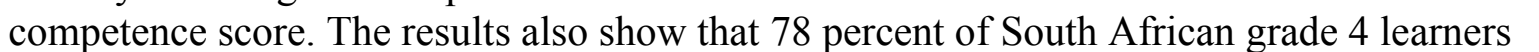

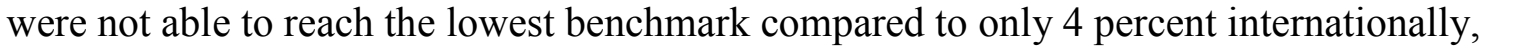

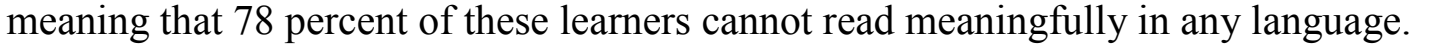

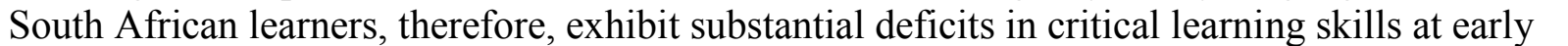

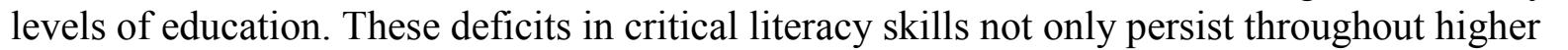

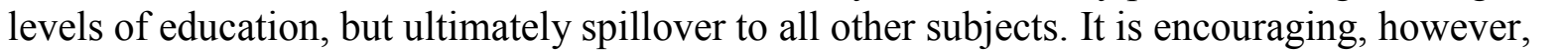
ए पामाप्त

$\square$

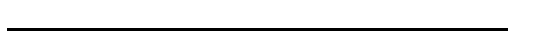

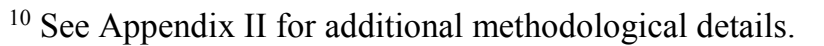

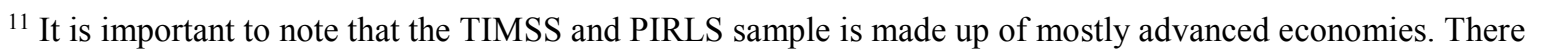

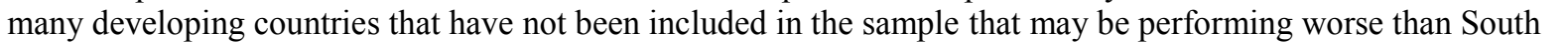

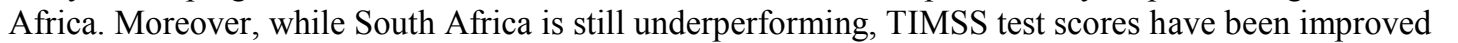
एण口冋एणा 


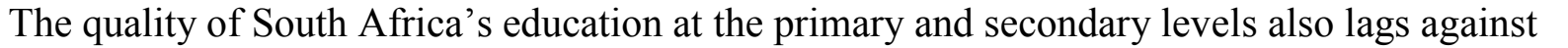

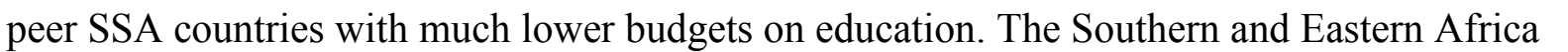

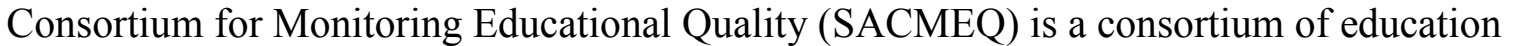

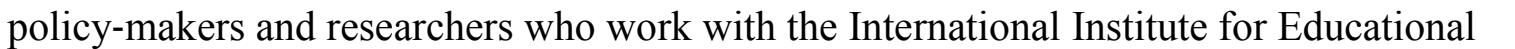

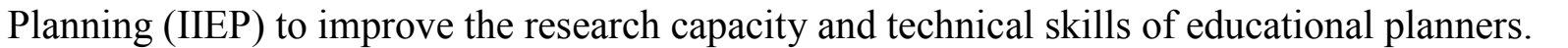

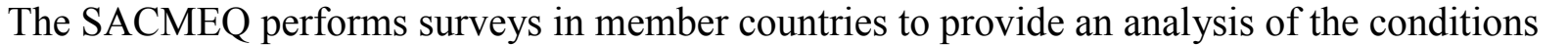

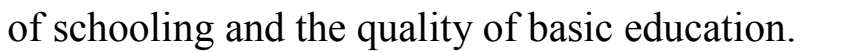

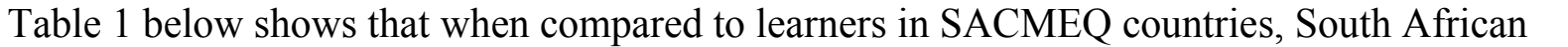

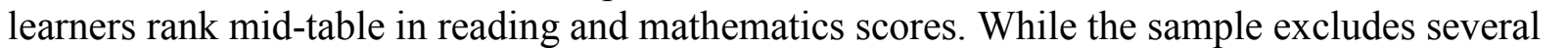

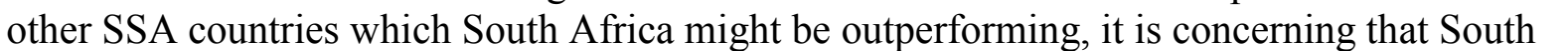

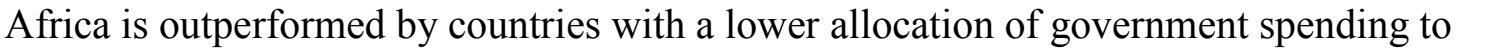

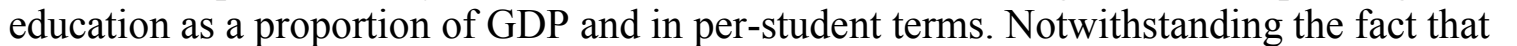

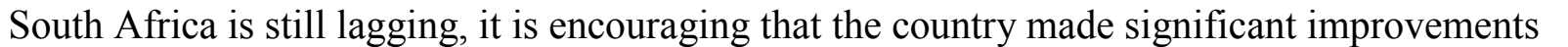

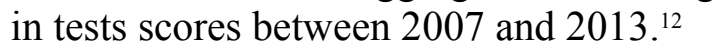

\begin{tabular}{|c|c|c|c|c|c|c|}
\hline \multicolumn{7}{|c|}{ Table 1. SACMEQ $2007 \& 2013$ Test Results } \\
\hline \multirow[t]{2}{*}{ Country } & \multicolumn{3}{|c|}{ Learner reading score } & \multicolumn{3}{|c|}{ Learner mathematics score } \\
\hline & 2007 & 2013 & Diff & 2007 & 2013 & Diff \\
\hline$\square \square \square\|\| \square \square$ & $\square \square$ & $\square \square$ & $\square$ & $\square \square$ & $\square \square \square$ & $\square \square$ \\
\hline$\square \square \| \mathbb{D}$ & $\square \square$ & $\square \square$ & $\square$ & $\square \square$ & $\square \square$ & $\square$ \\
\hline$\square\|\|\|\|\|\| \|$ & $\square \square$ & $\square \square$ & $\square$ & $\square \square$ & $\square \square$ & $\square$ \\
\hline$\square \square \square\|\| \| \square$ & $\square \square$ & $\square \square$ & $\square$ & $\square \square$ & $\square \square$ & $\square \square$ \\
\hline$\square \square \square \square \square \square$ & $\square \square$ & $\square \square$ & $\square$ & $\square \square$ & $\square \square$ & $\square$ \\
\hline South Africa & 495 & 558 & 63 & 495 & 587 & 92 \\
\hline$\square \square\|\| \square$ & $\square \amalg$ & $\square \square$ & $\square$ & $\square \square$ & $\square \square$ & $\square \square$ \\
\hline$\square \square \square \| \square \square$ & $\square \square$ & $\square \square$ & $\square$ & $\square \square$ & $\square \square$ & $\square$ \\
\hline$\square \square\|\| \| \square$ & $\square \square$ & $\square \square$ & $\square$ & $\square \square$ & $\square \square$ & $\square \square$ \\
\hline 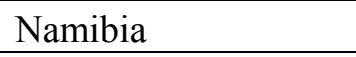 & $\square \square$ & $\square \square$ & $\square \square$ & $\square \square$ & $\square \square$ & $\square \square$ \\
\hline$\square \square \| \square \square \square \square$ & $\square \square$ & $\square \square$ & $\square$ & $\square \square$ & $\square \square$ & $\square \square$ \\
\hline$\square \square \square \square$ & $\square \amalg$ & $\square \square$ & $\square$ & $\square \square$ & $\square \square$ & $\square \square$ \\
\hline$\square \square \square \square$ & $\square \square$ & $\square \square$ & $\square$ & $\square \square$ & $\square \square$ & $\square \square$ \\
\hline SACMEQ (Average) & 500 & 507 & 7 & 507 & 584 & 77 \\
\hline
\end{tabular}

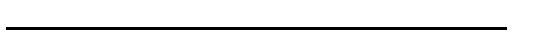

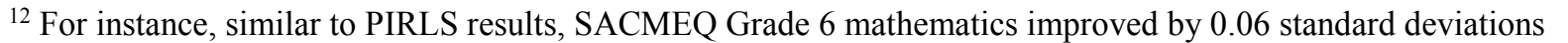
पापा山ापा 
Figure 6. South Africa's Grade 5 Scores versus International Grade 4 Scores in Mathematics

Russian Federation

Ireland

England

Portuga

United States

Finland

Netherlands

Czech Republic

Cyprus

Slovenia

Serbia

Canada

Spain

Centre Point

New Zealand
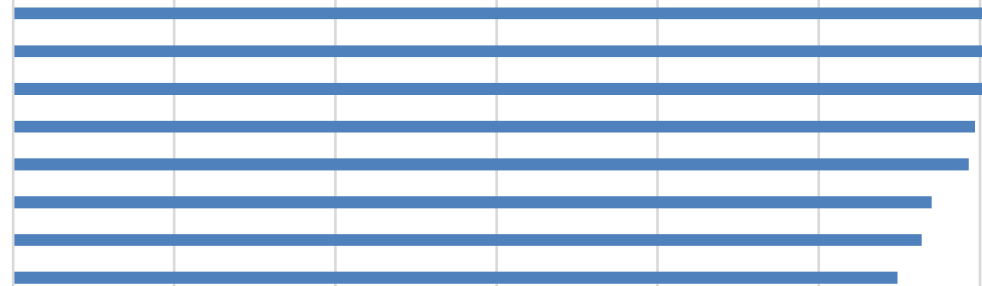

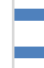

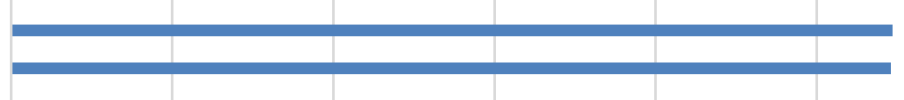

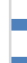
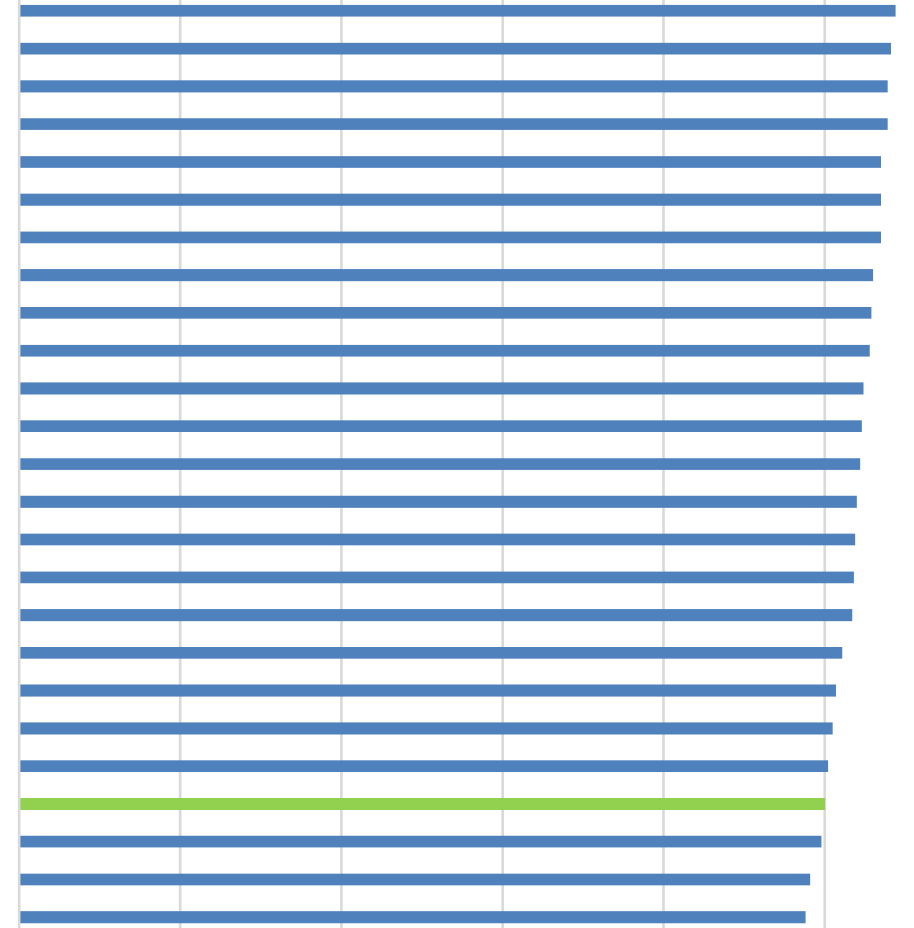

Turkey

Chile
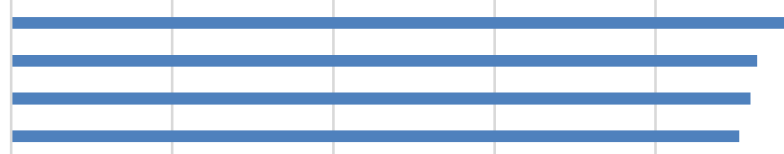

Bahrain

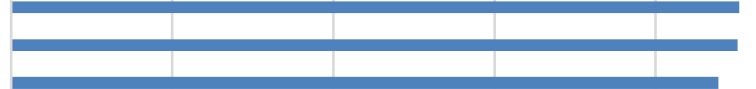

n
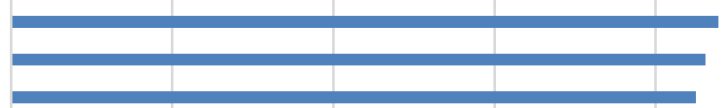

Minimum...

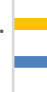

Jordan

Morocco

Kuwait

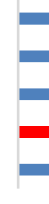

0

100

200

300

400

500

600

700 


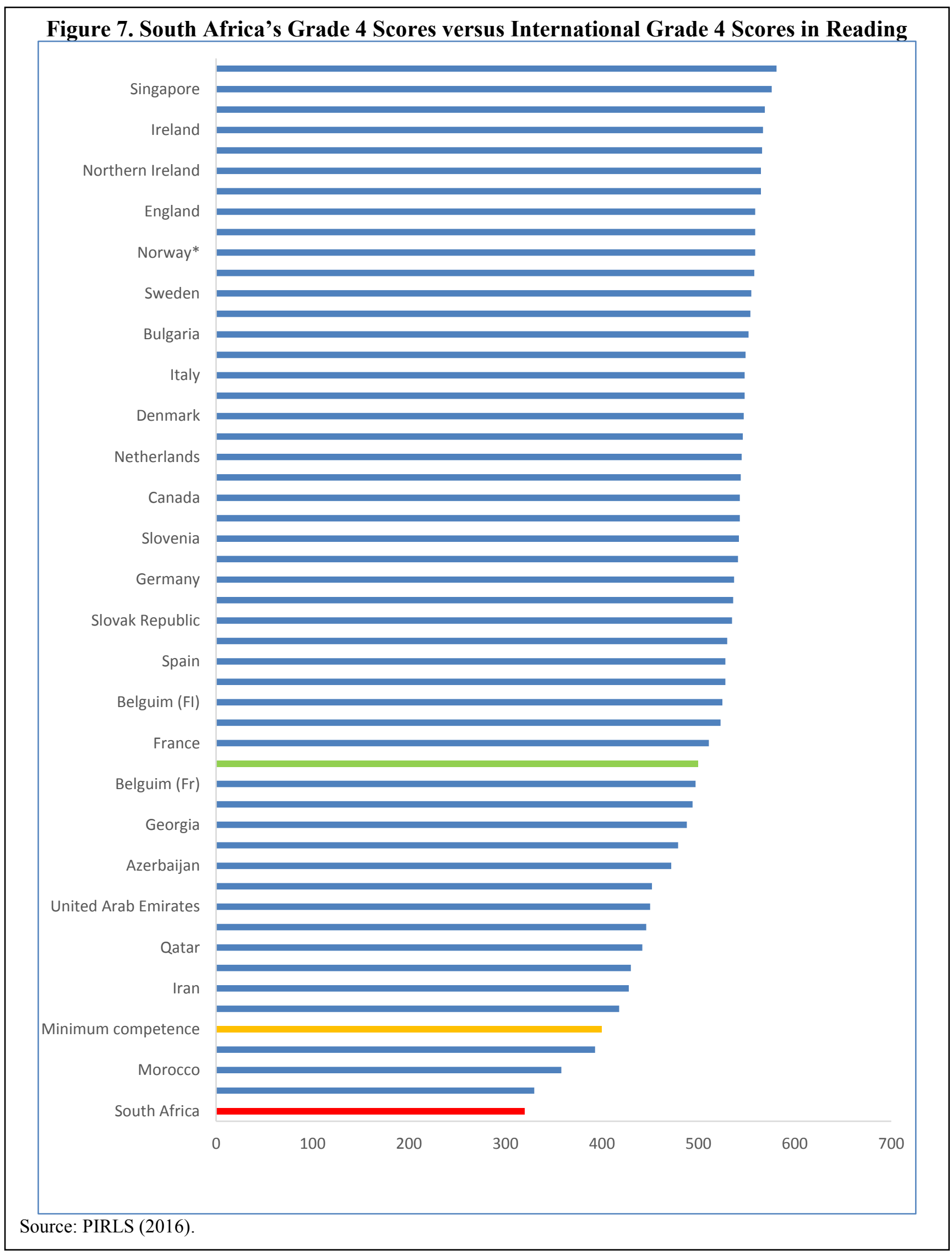




\section{B. Secondary School Level}

\section{Input indicators}

$\square$

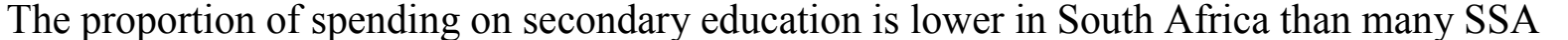

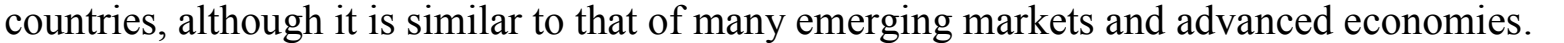

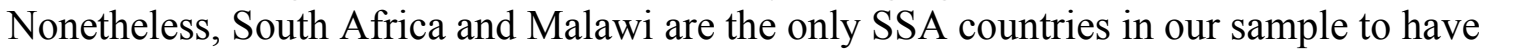

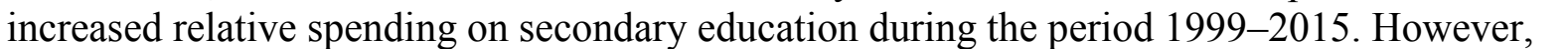

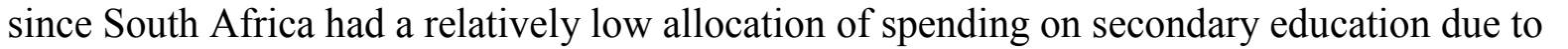

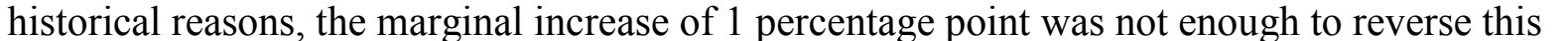
trend. The fact that South Africa's secondary education expenditure is comparatively low may

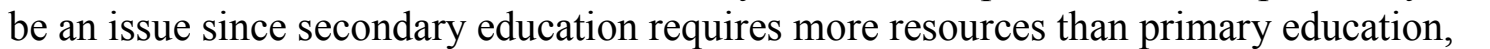

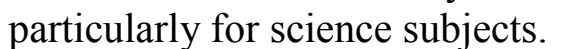

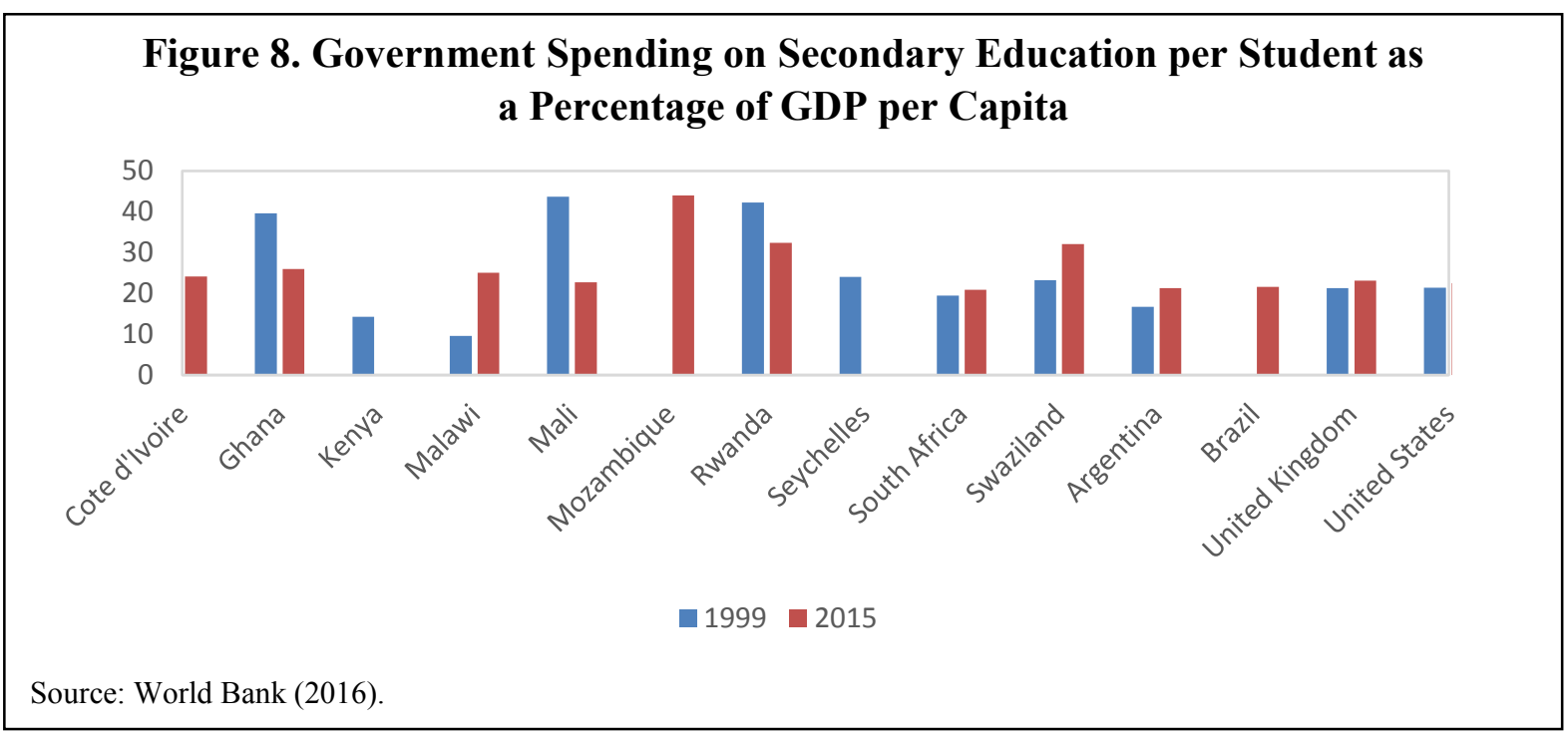

\section{Enrollment indicators}

Low retention rates in South Africa's education system start in lower secondary school

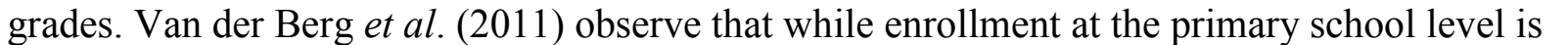

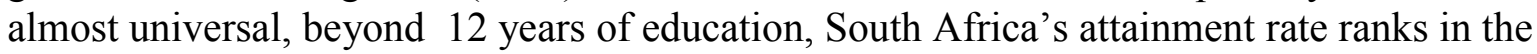

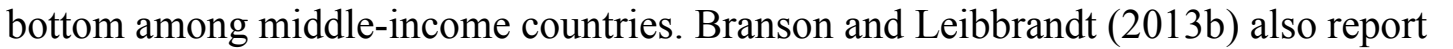

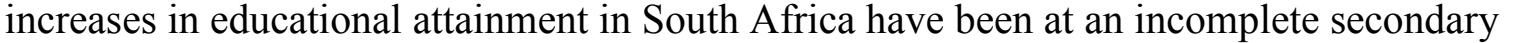

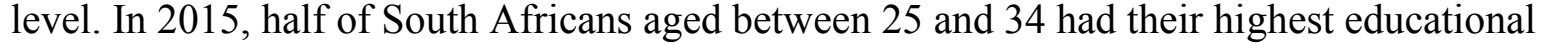

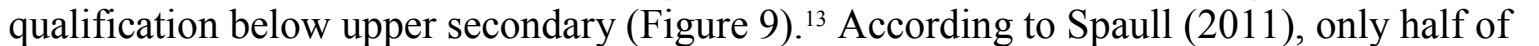

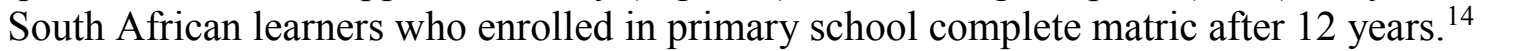

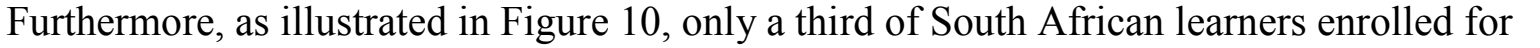

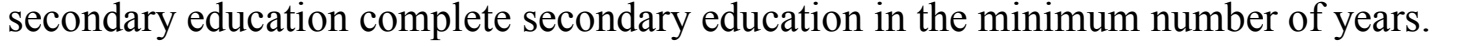

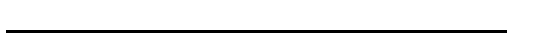

पா घण

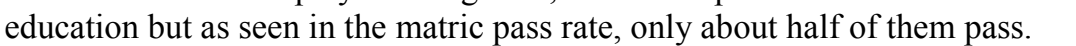

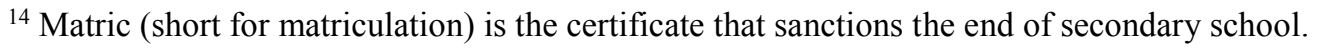




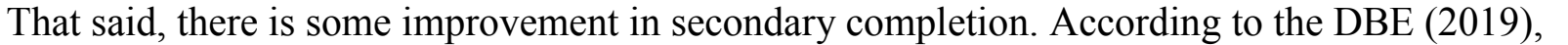

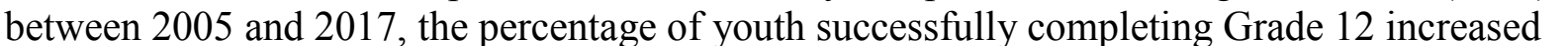

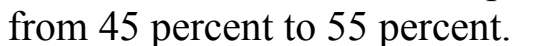
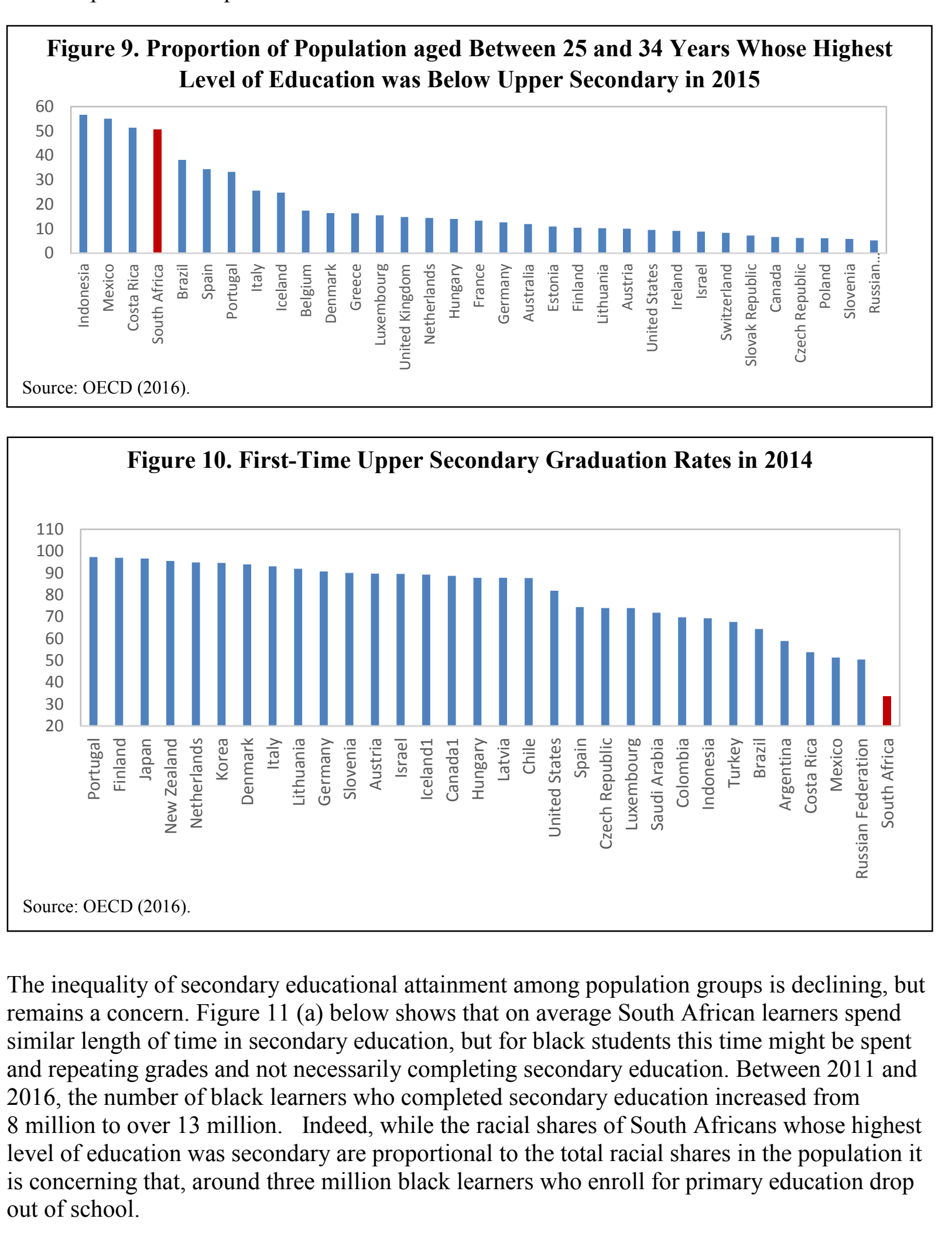

CInternational Monetary Fund. Not for Redistribution 
Figure 11 (a). South Africa's Average Years of Secondary Schooling by Population Group in 2016

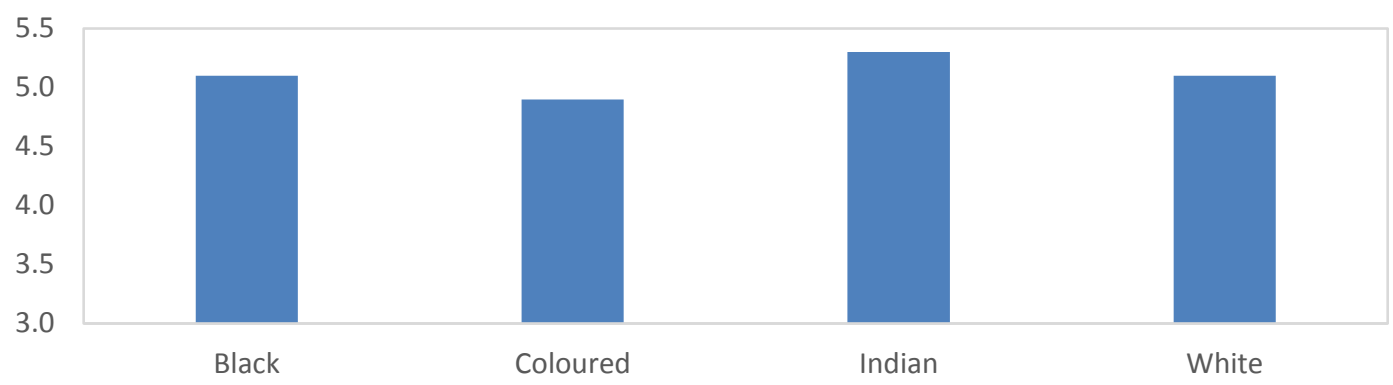

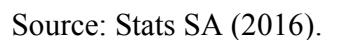

Figure 11 (b). South Africa's Proportion of Individuals Aged 25-64 Whose Highest Level of Education was Secondary Education in 2016

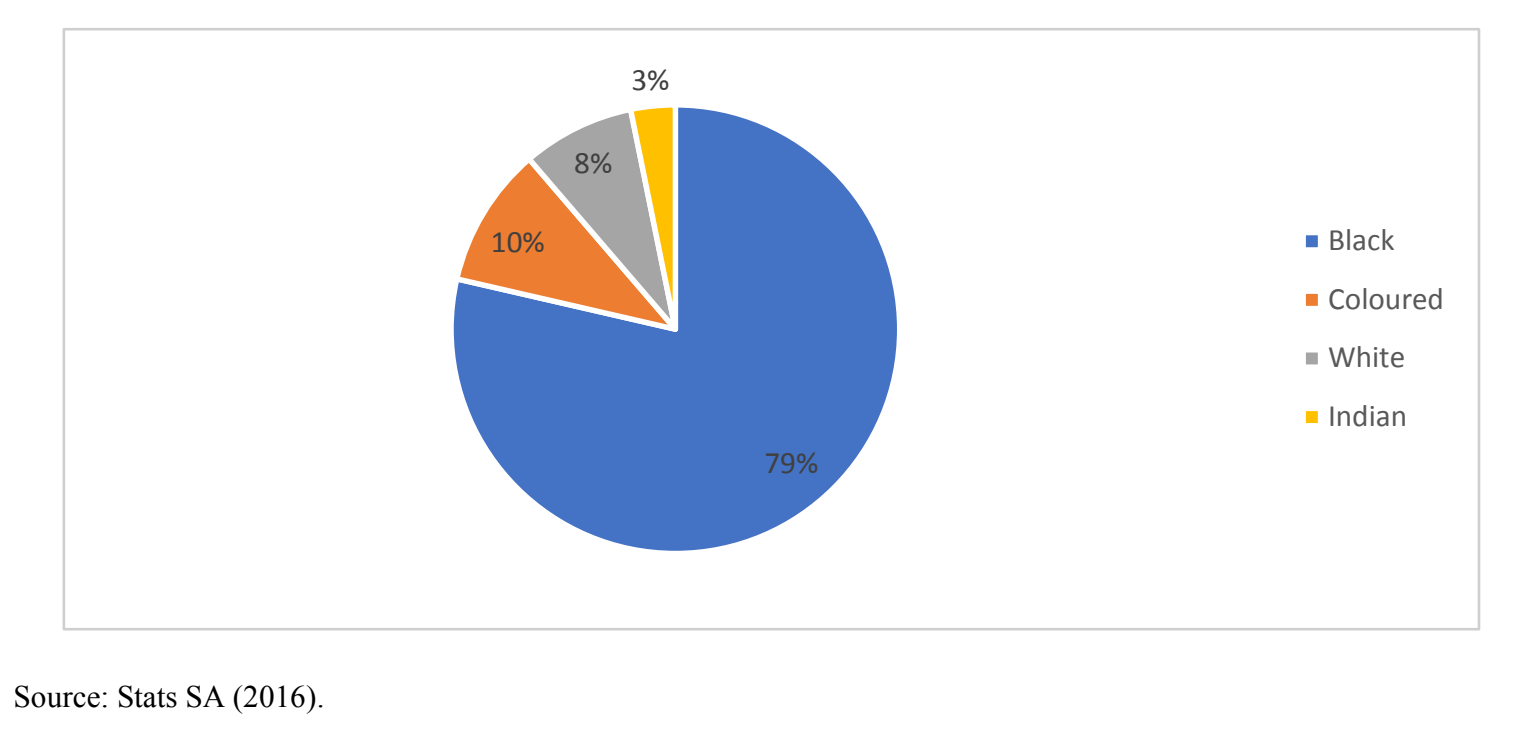

\section{Quality indicators}

\section{$\square$

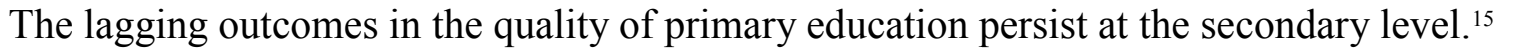

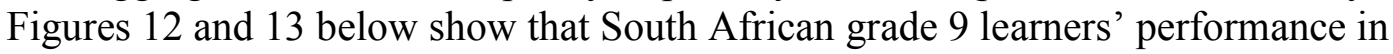

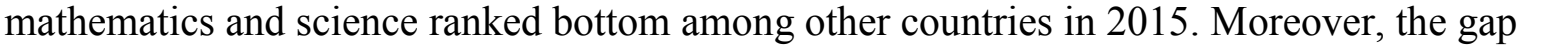

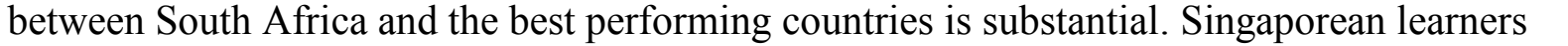

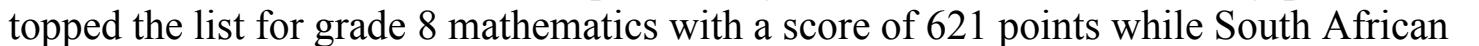

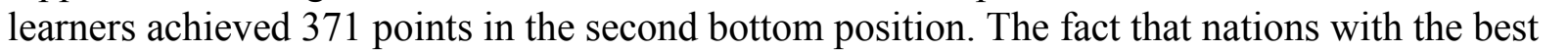

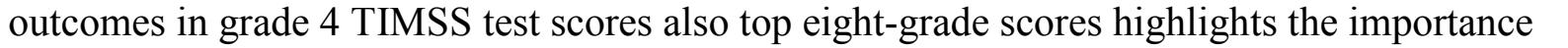

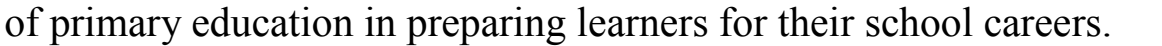 \\ $\square$ mmmmmmmmmmmm

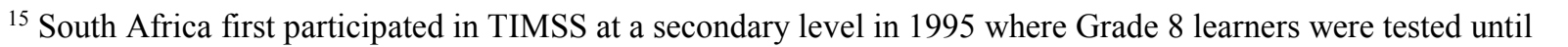

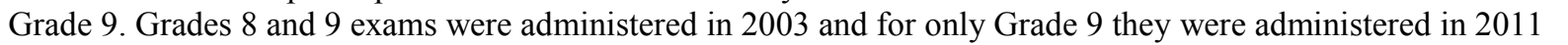

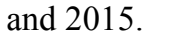




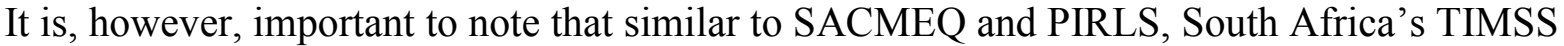

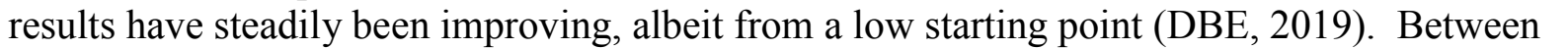

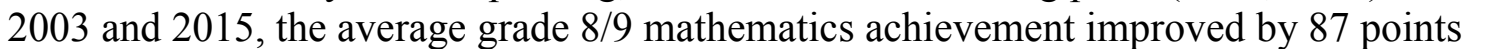

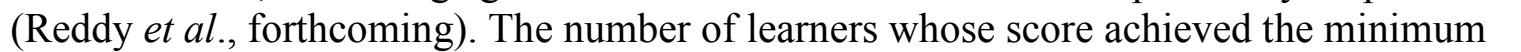

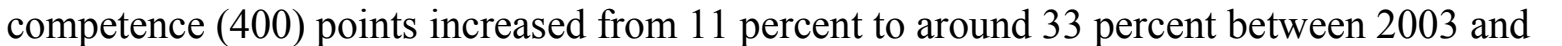

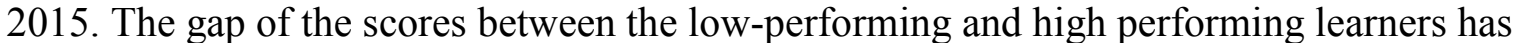

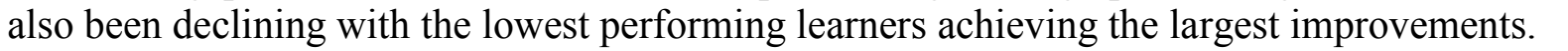

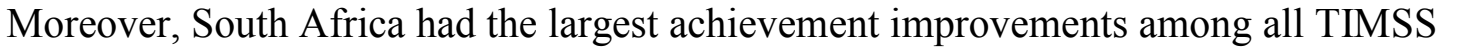

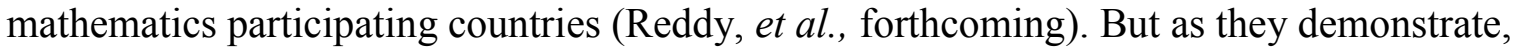

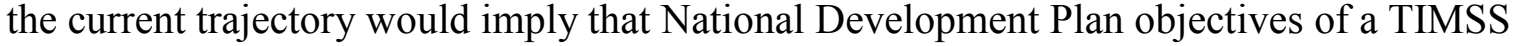

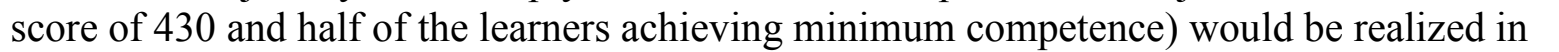

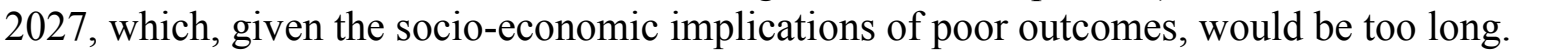
$\square$

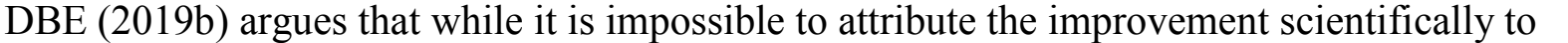

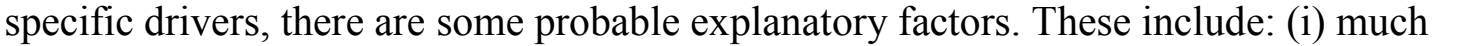

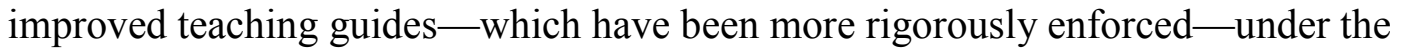

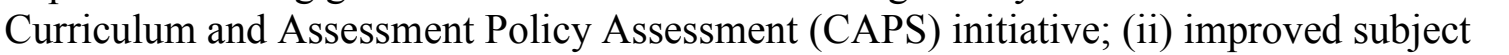

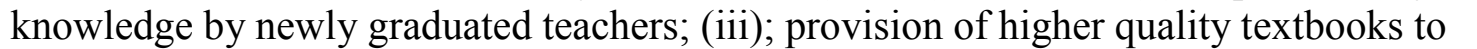

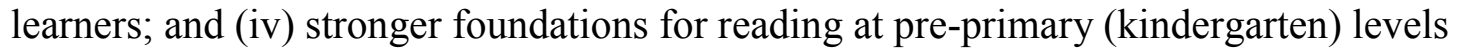

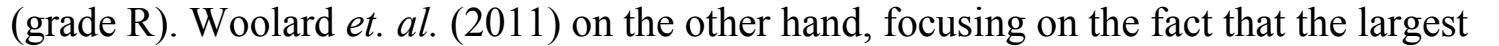

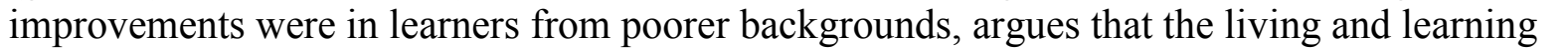

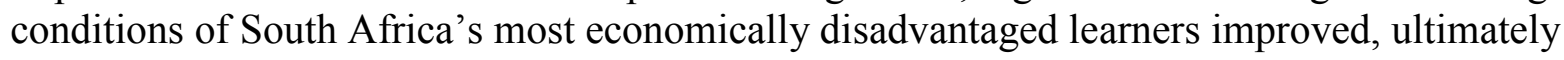

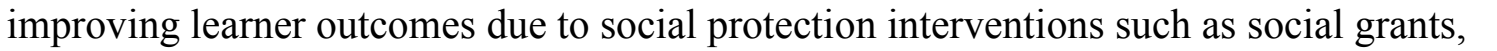

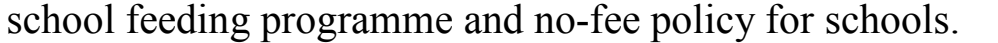

$\square$ 
Figure 12. South Africa's Grade 9 Scores versus International Grade 8 Scores in Mathematics

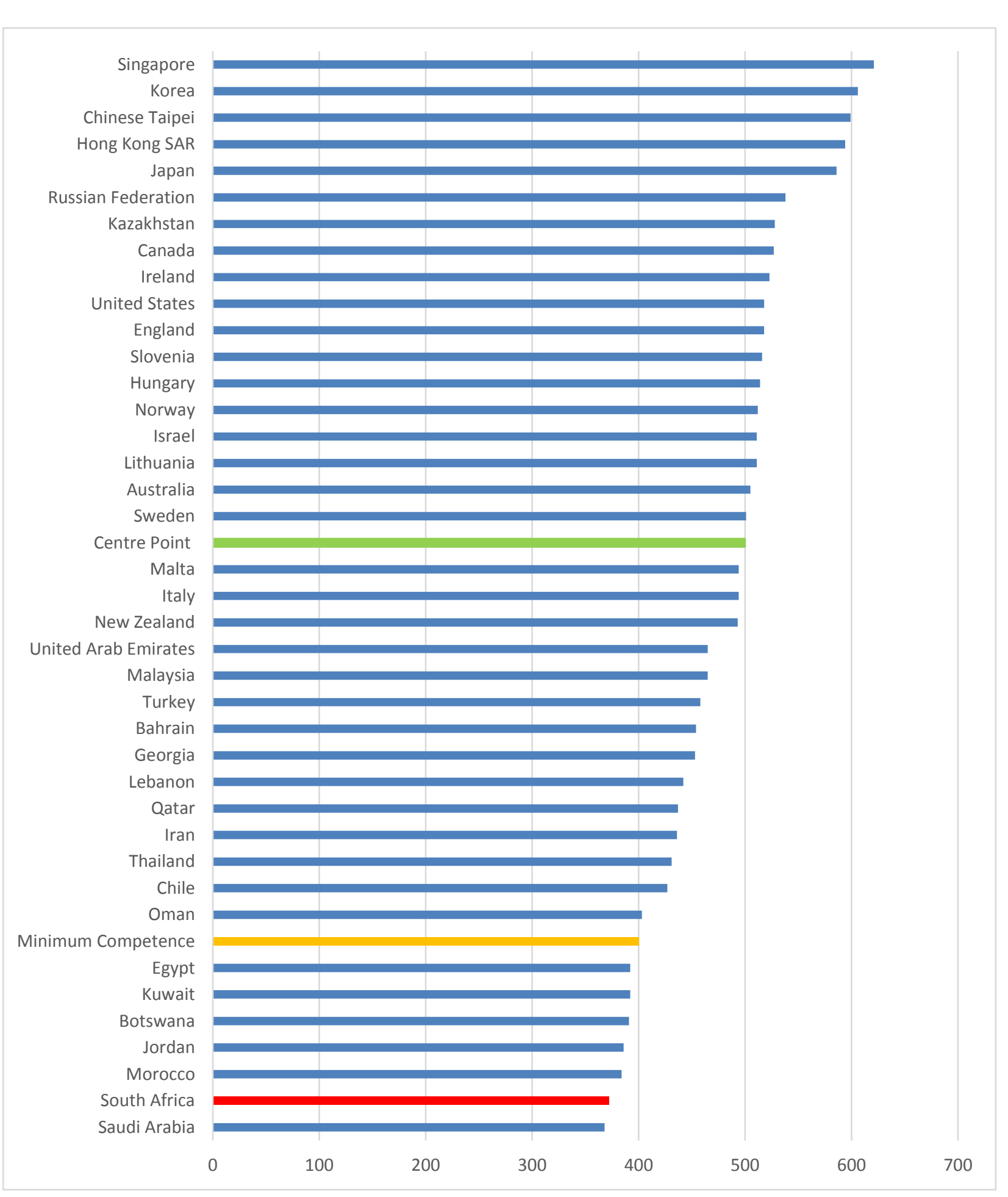


Figure 13. South Africa's Grade 9 Science Scores versus Grade 8 International Science Score

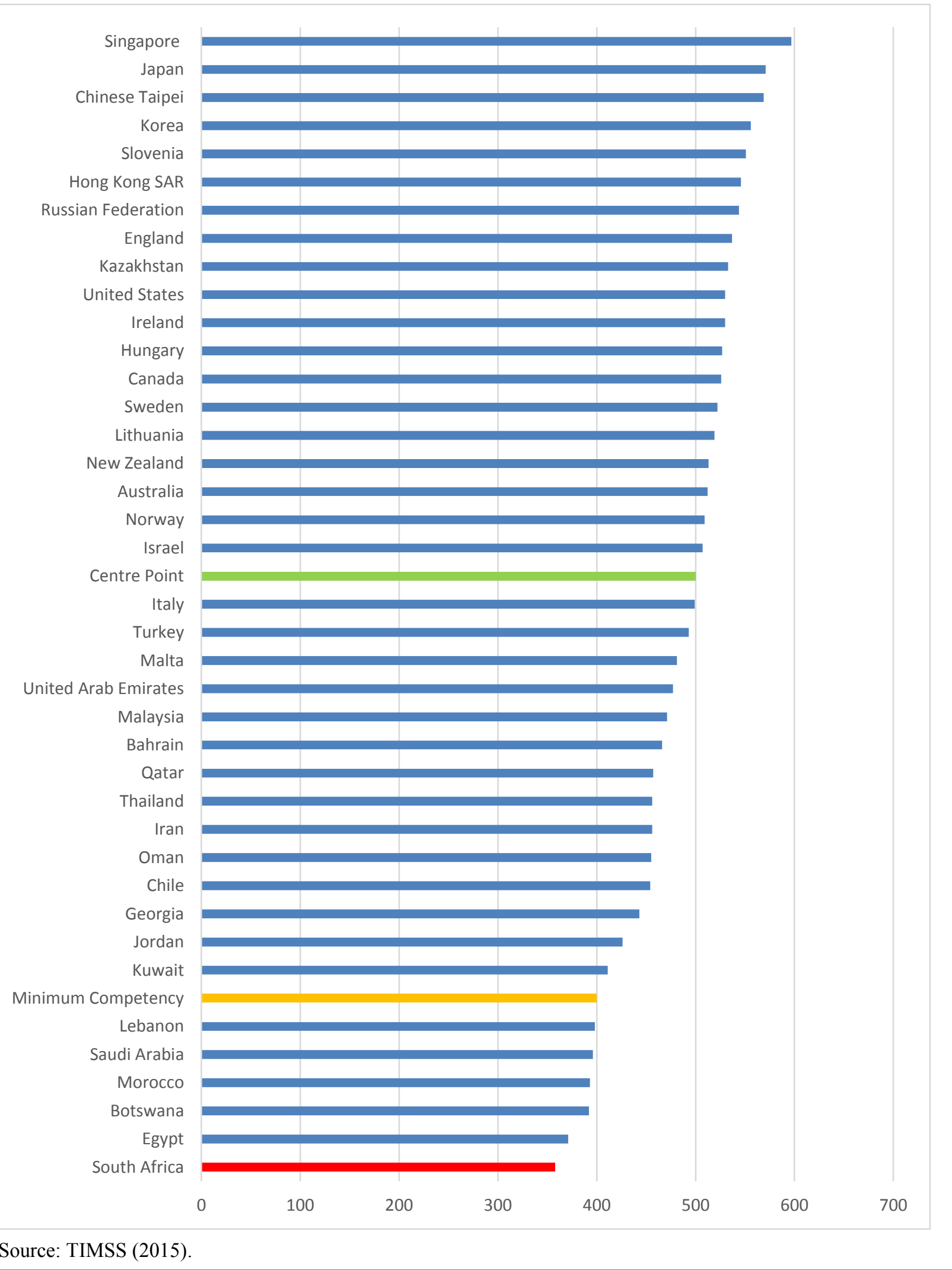


Evidence of South Africa's lagging quality of basic education can also be seen in the

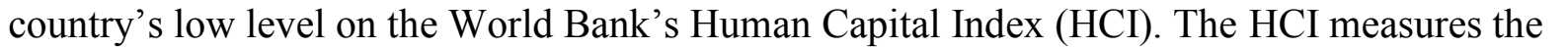

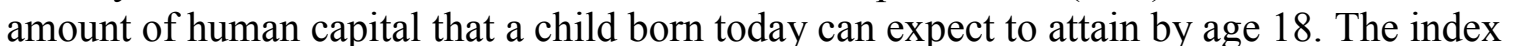

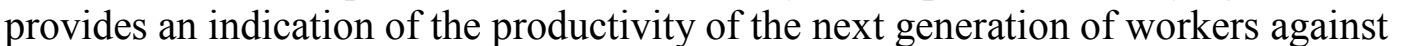

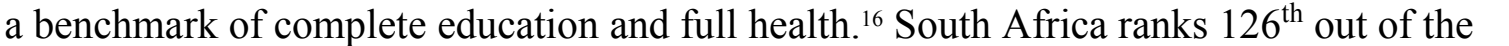

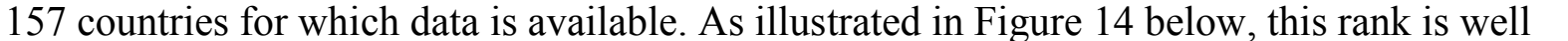

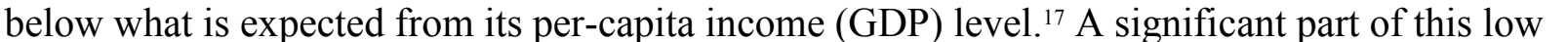

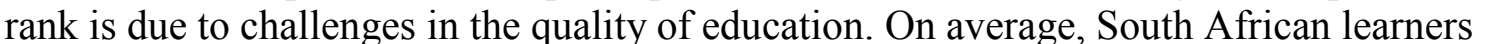

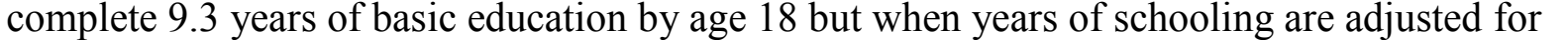

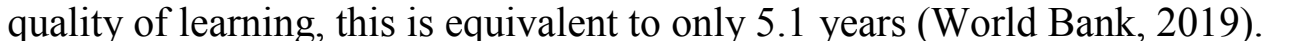

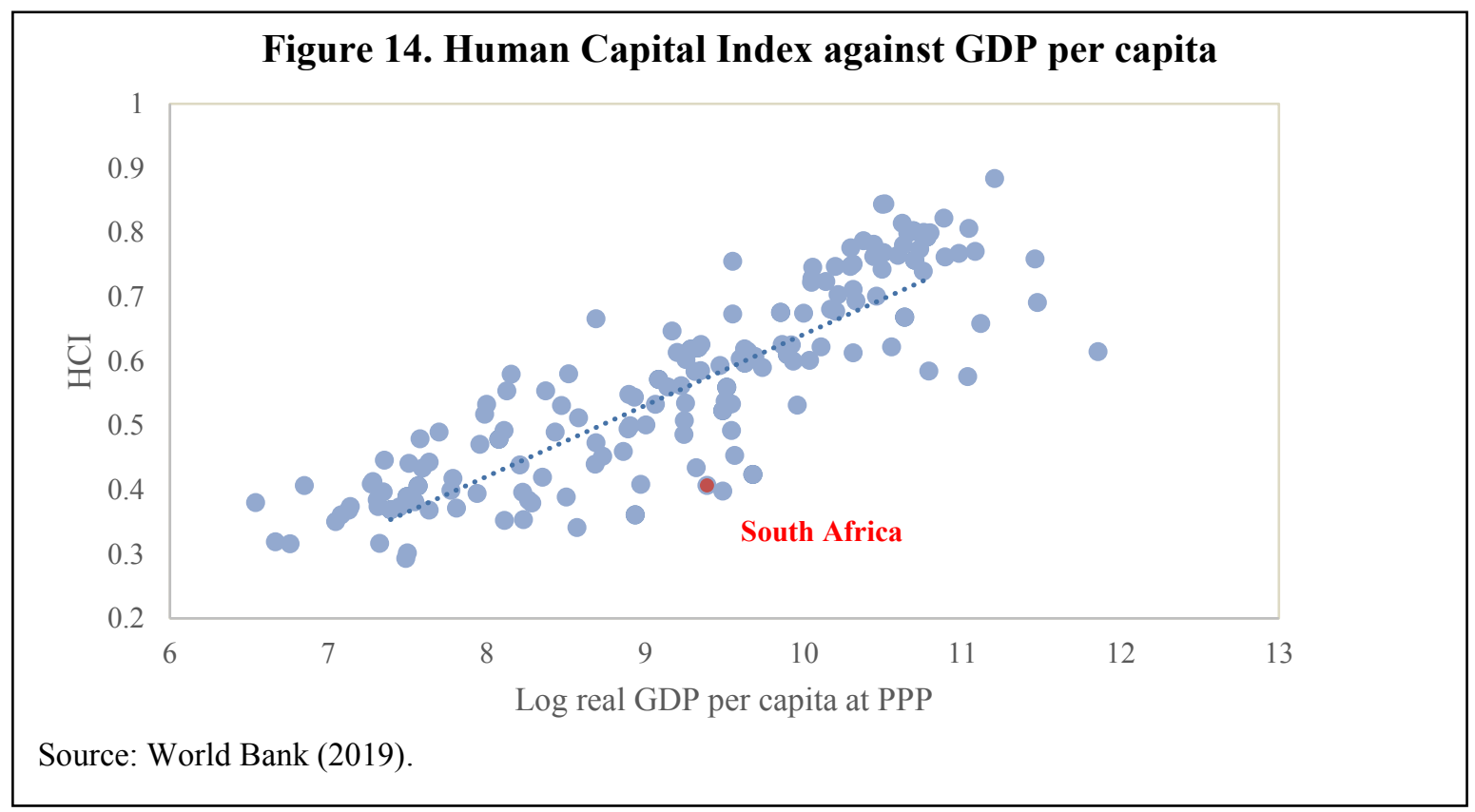

C. Tertiary Education Level

\section{Input indicators}

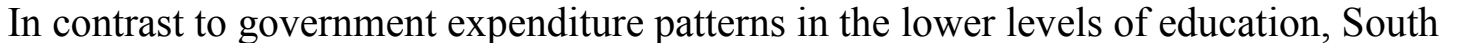

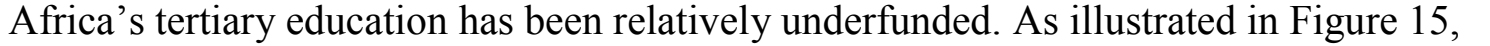

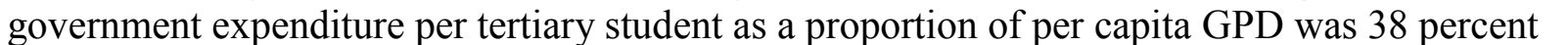

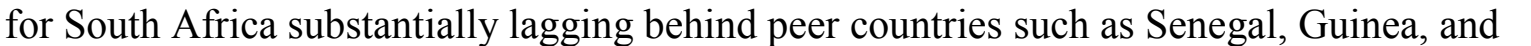

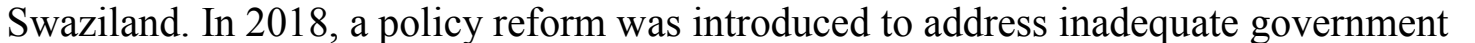

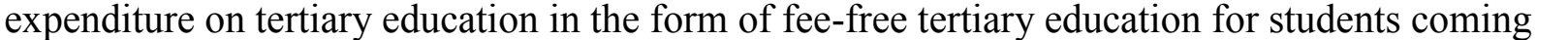

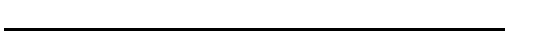

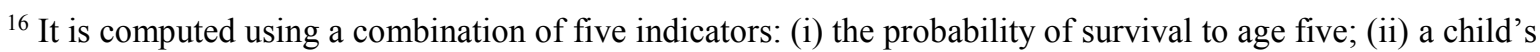

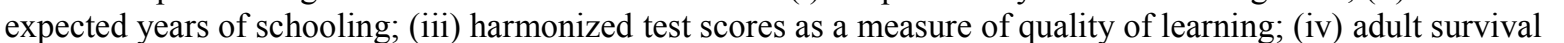

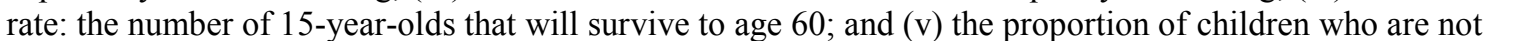

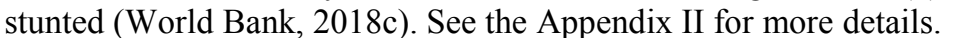

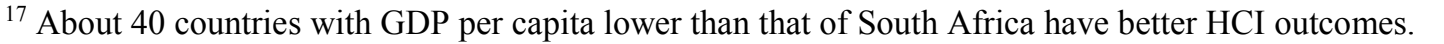




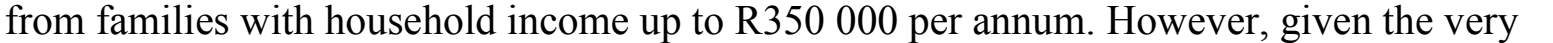

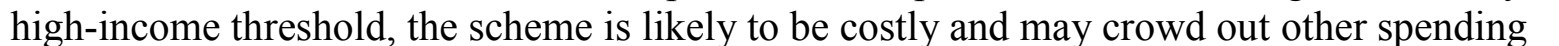

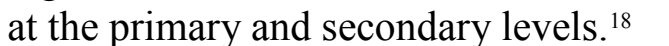

Figure 15. Government Expenditure per Tertiary Student as a Percentage of GDP per Capita in 2014

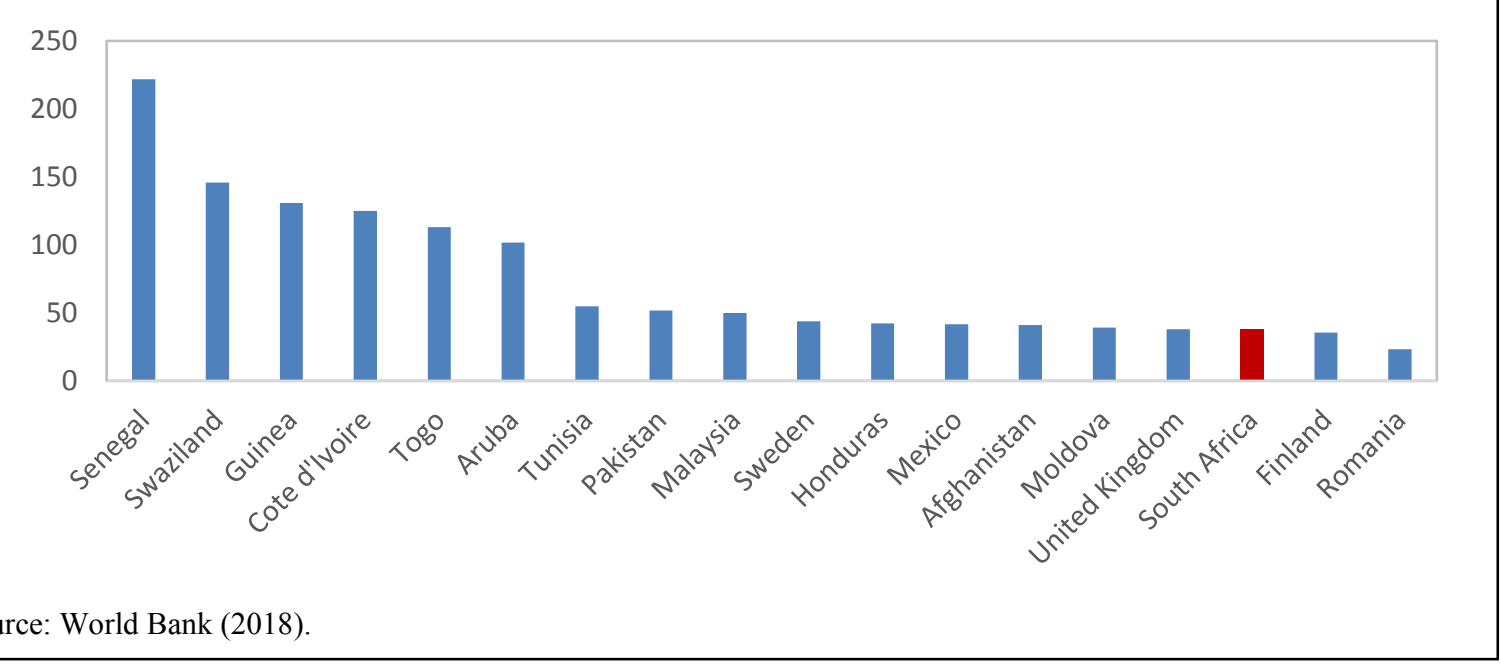

$\square$

\section{Enrollment indicators}

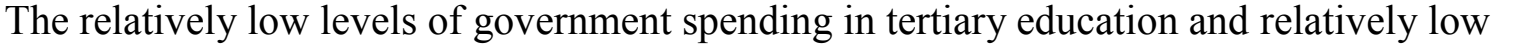

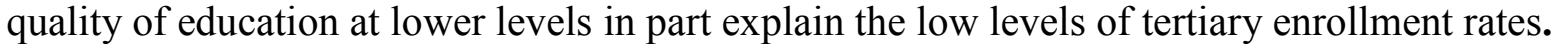

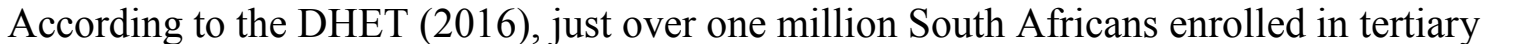

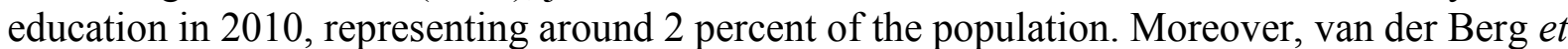

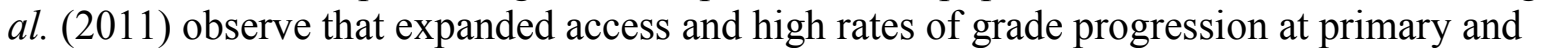

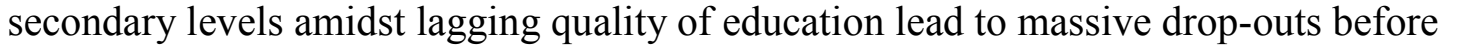

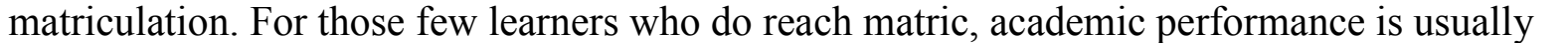

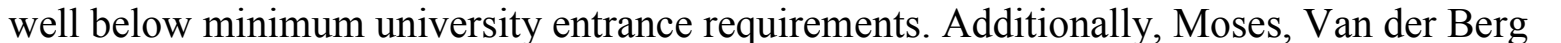

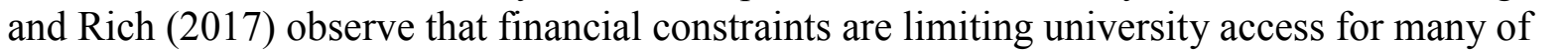
South Africa's learners.

$\square$

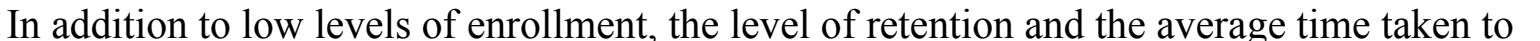

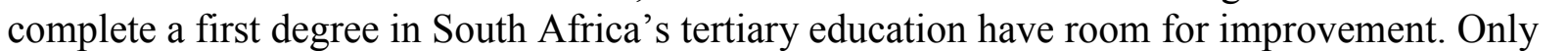

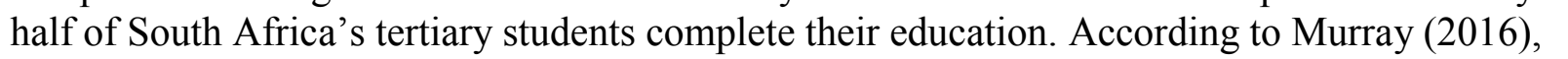

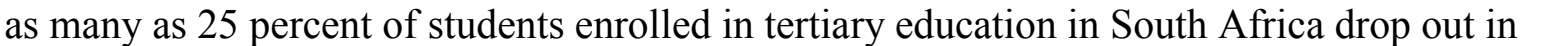
ए

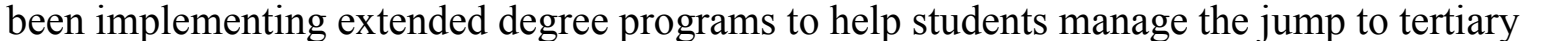

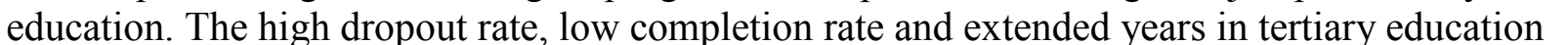
all undermine the efficiency of expenditure in South Africa's tertiary education. Figure $\square \square$

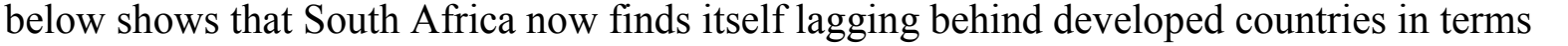

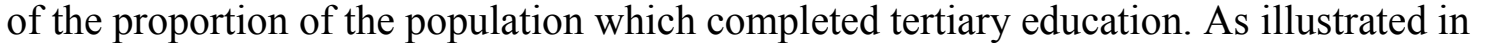

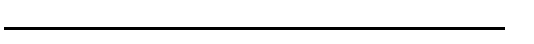

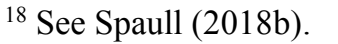


$\square \square \square-2$ below, the completion rates in South Africa's tertiary education are well below the

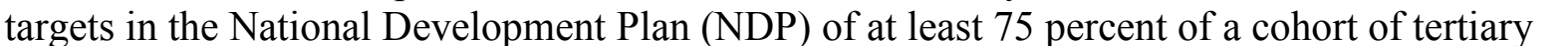

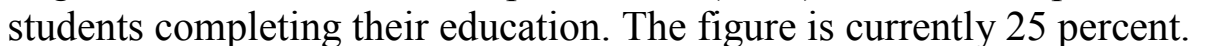

\section{Figure 16. Proportion of Population Aged between 25 and 64 Years with Tertiary Education in 2015}

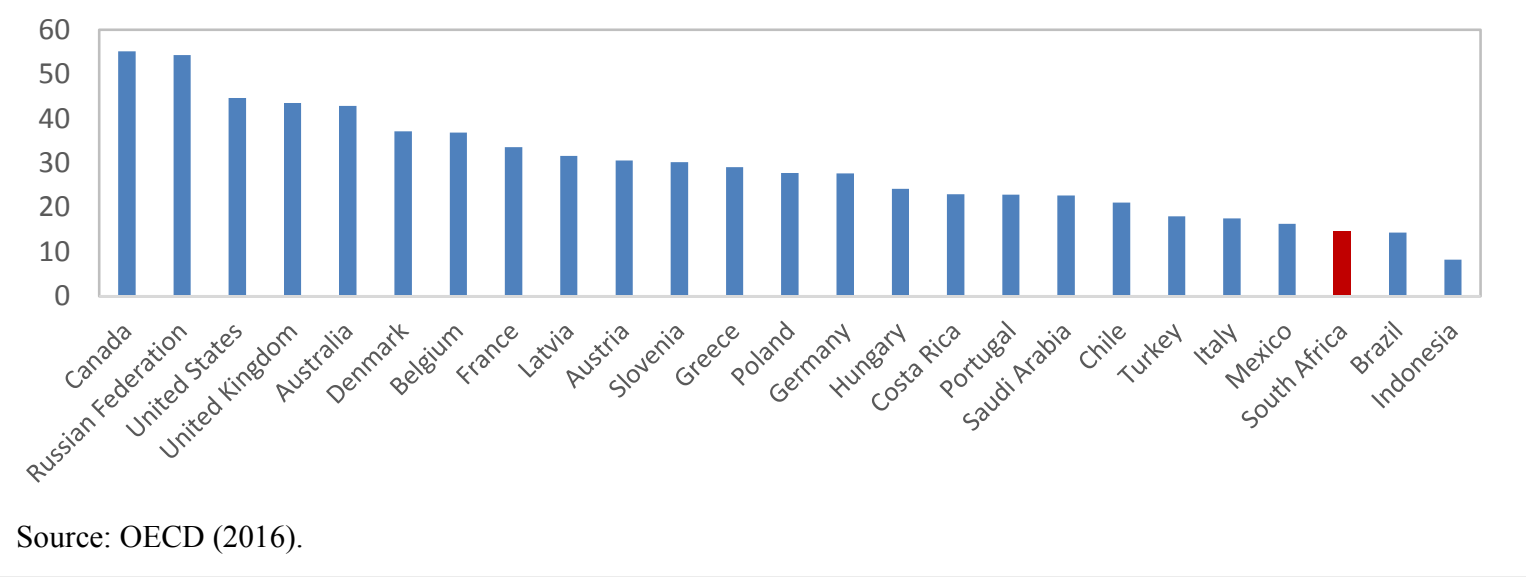

$\square$

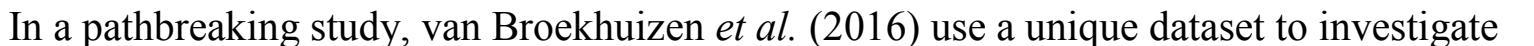

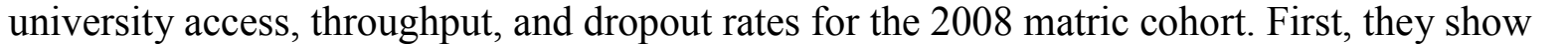
ए

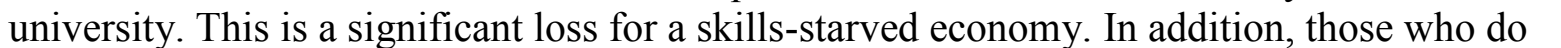

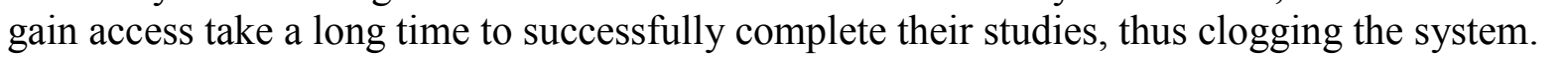

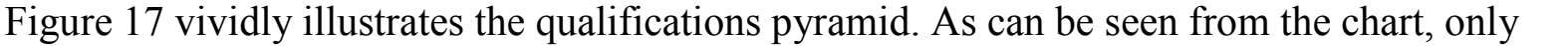

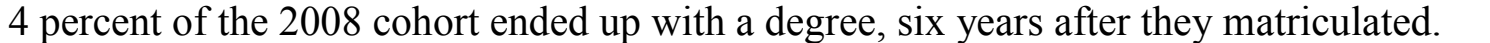

\begin{tabular}{|c|c|c|}
\hline \multicolumn{3}{|c|}{$\begin{array}{l}\text { Table 2. South Africa's Summaries of Graduation Rates in Public Higher } \\
\text { Education Institutions in } 2015 \text { (in percent) }\end{array}$} \\
\hline$\square$ & $\square \square\|\|\|\|\|\|$ & 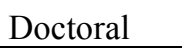 \\
\hline 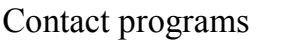 & $\square \square$ & $\square \square$ \\
\hline 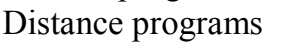 & $\square$ & $\square$ \\
\hline
\end{tabular}




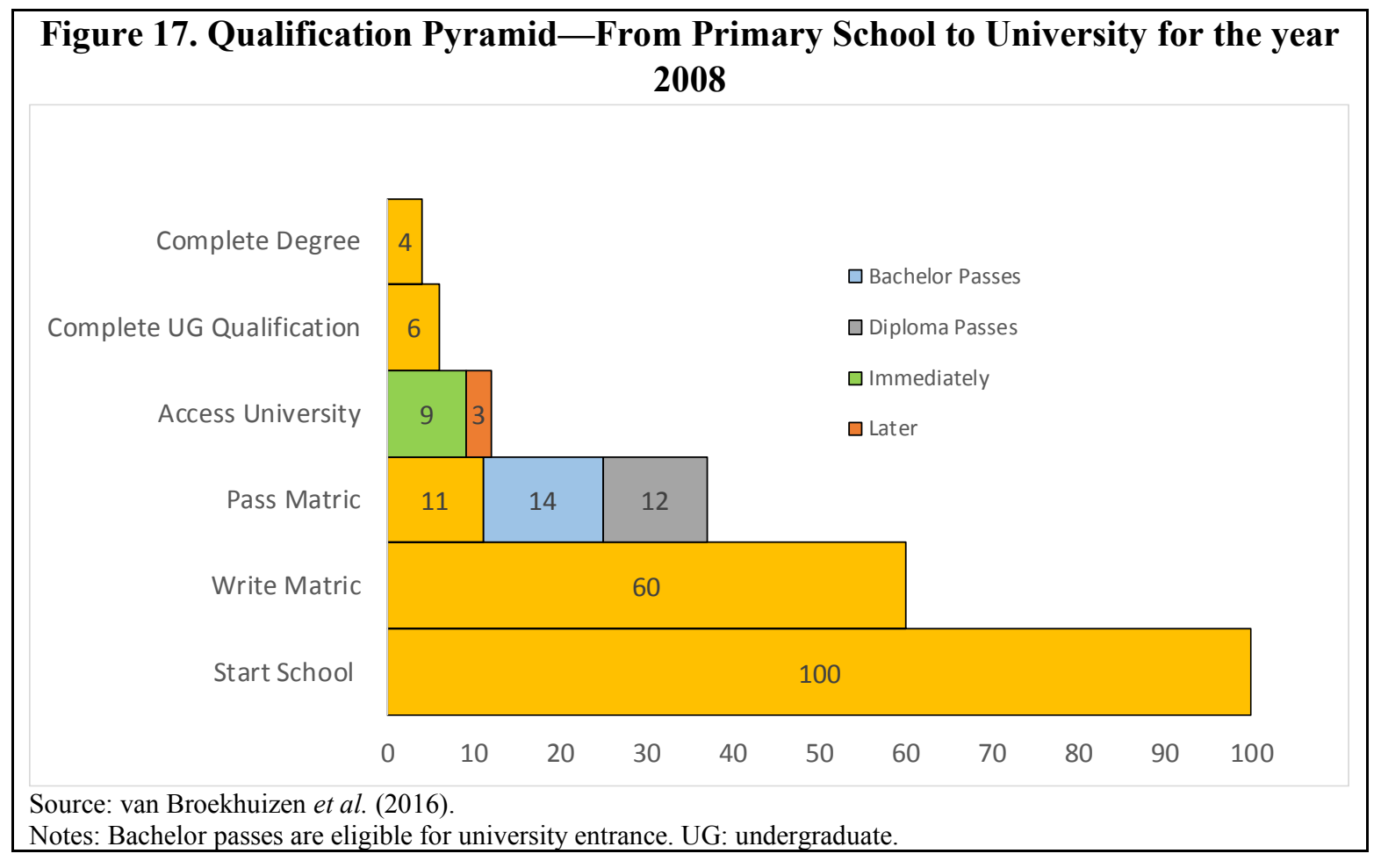

There are substantial racial disparities in retention in South Africa’s tertiary education.

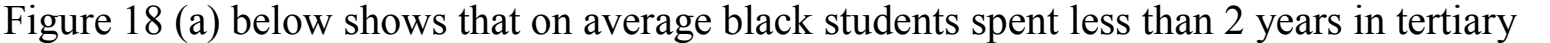

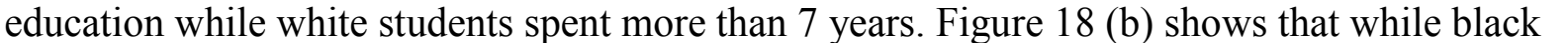

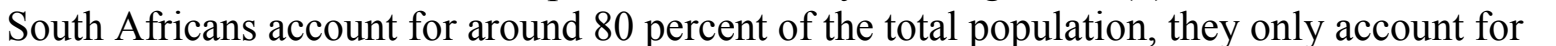

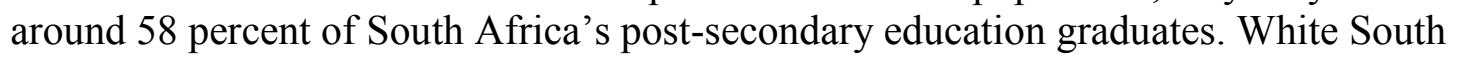

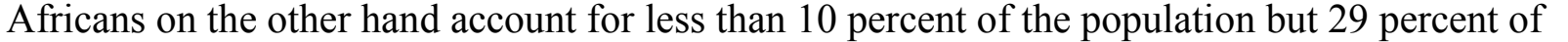

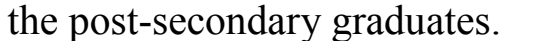

$\square$

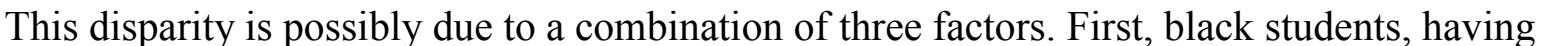

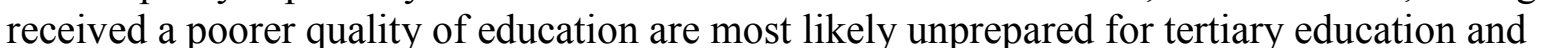

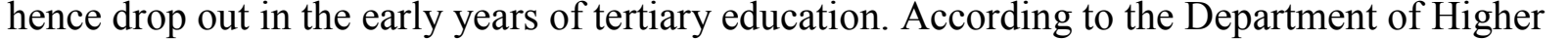

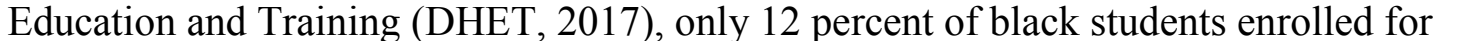

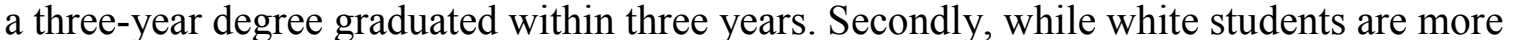

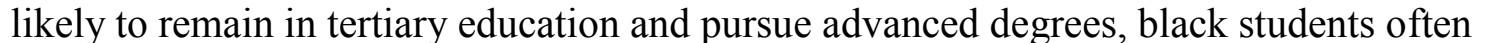

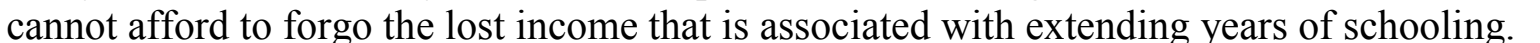

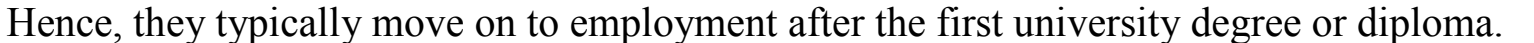

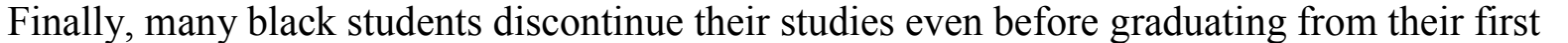

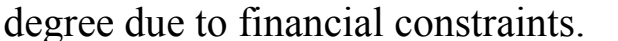

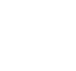




\section{Figure 18 (a). South Africa's Average Years of Schooling Post-Secondary by Population Group in 2016}

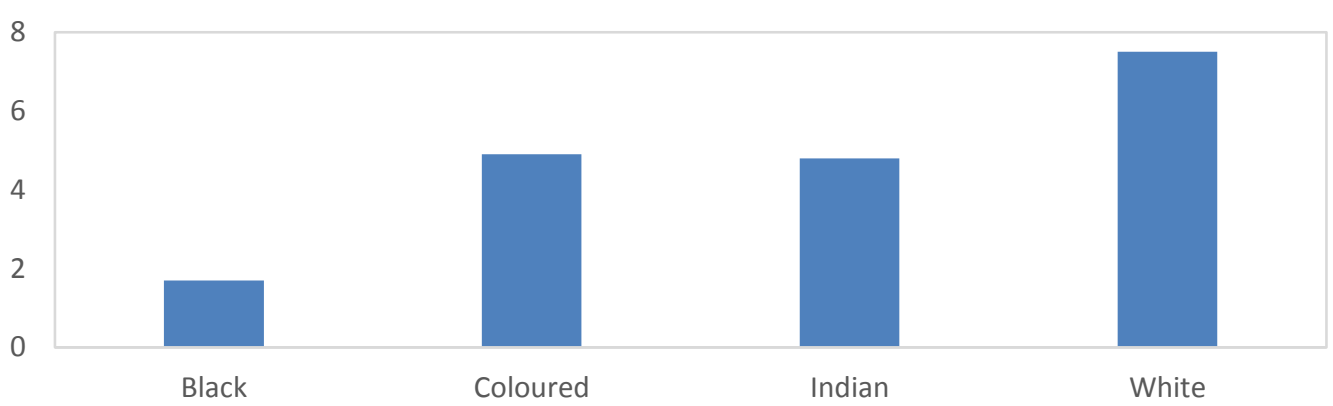

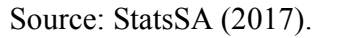

Figure 18 (b). South Africa's Proportion of Individuals aged 25-64 Whose Highest Level of Education was Tertiary Education in 2016

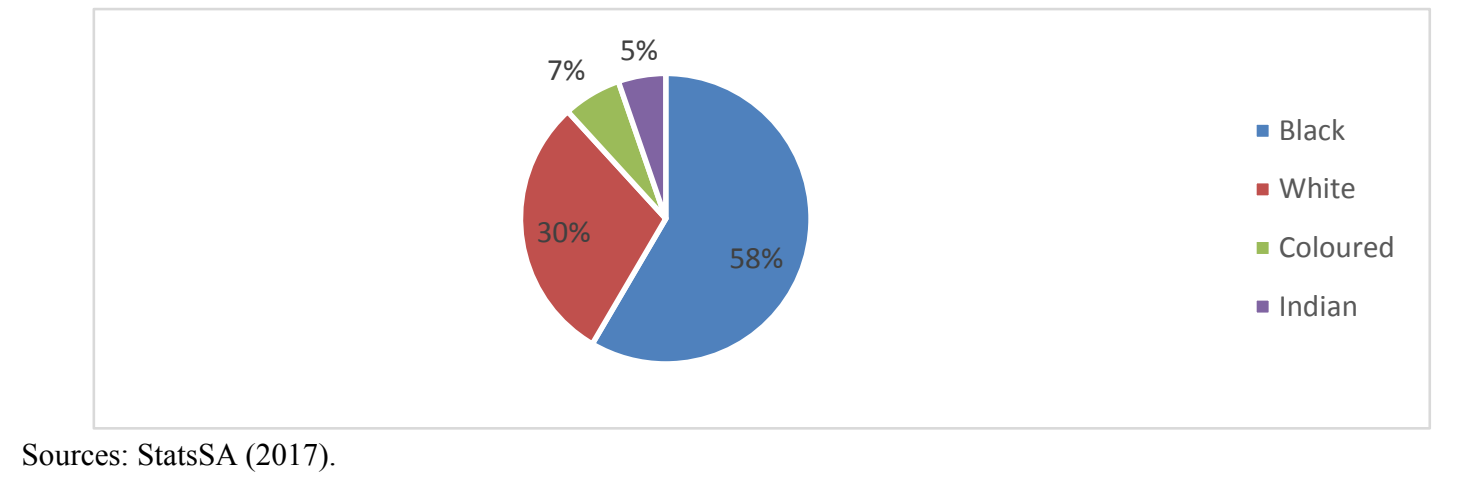

\section{Quality Indicators}

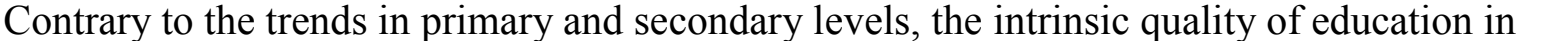

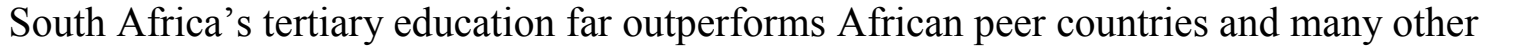

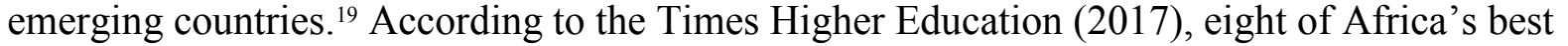

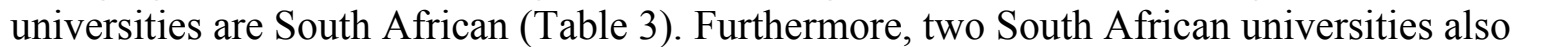

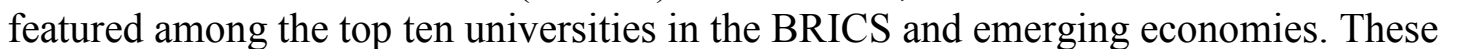

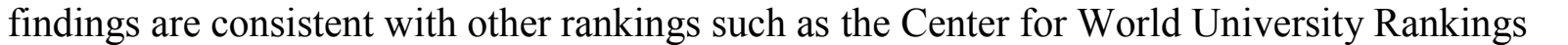

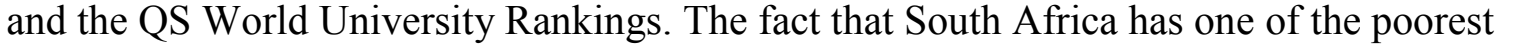

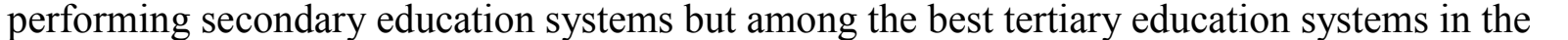

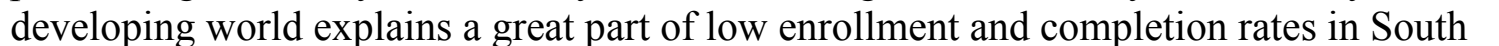

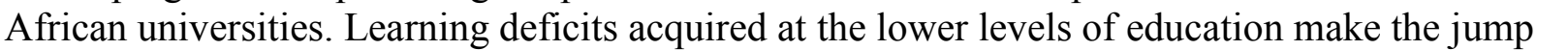
to university too high for many of South Africa's students.

\footnotetext{
世mmmmmmmmmmmmmmm

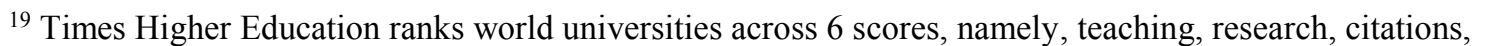
ए

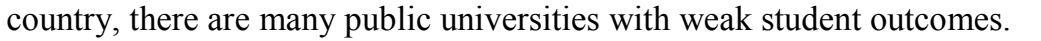




\begin{tabular}{|c|c|c|}
\hline \multicolumn{3}{|c|}{ Table 3. Times Higher Education African Universities Rankings in 2018} \\
\hline African Ranking & University & Country \\
\hline$\square$ & 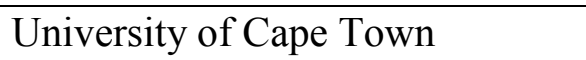 & 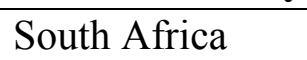 \\
\hline$\square$ & 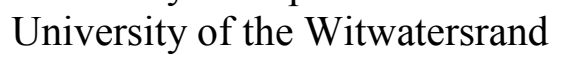 & 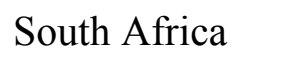 \\
\hline$\square$ & 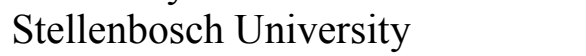 & 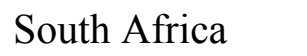 \\
\hline$\square$ & 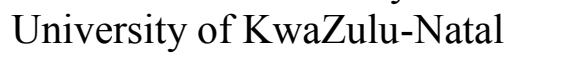 & 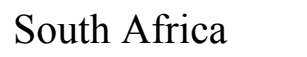 \\
\hline$\square$ & 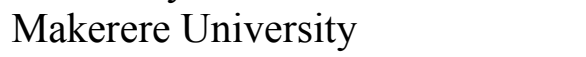 & $\square \square \| \square \square$ \\
\hline$\square$ & 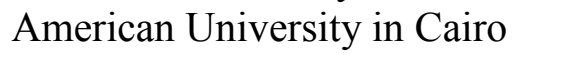 & $\square \square \square \square$ \\
\hline$\square$ & 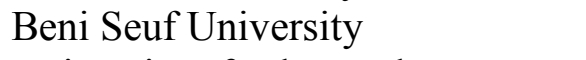 & $\square \square \square \square \square$ \\
\hline$\square$ & 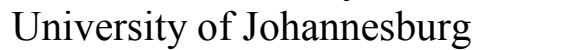 & 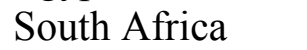 \\
\hline$\square$ & 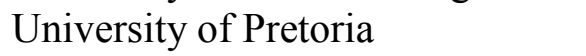 & 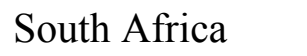 \\
\hline$\square \square$ & 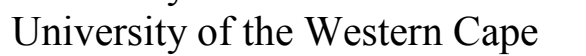 & 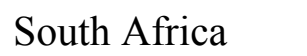 \\
\hline
\end{tabular}

\begin{tabular}{|c|c|c|}
\hline Ranking & University & Country \\
\hline$\square$ & 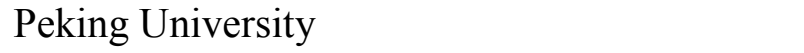 & $\square \square \square \square$ \\
\hline$\square$ & 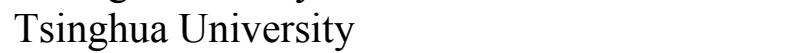 & पमाण \\
\hline$\square$ & 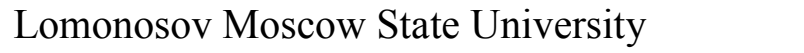 & $\square \square\|\| \square$ \\
\hline$\square$ & 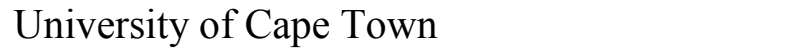 & 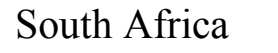 \\
\hline$\square$ & 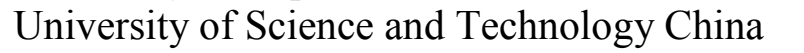 & $\square \square \| \square$ \\
\hline$\square$ & 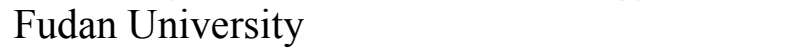 & $\square \square \| \square$ \\
\hline$\square$ & 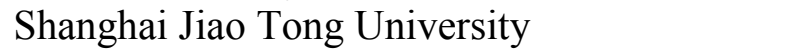 & $\square \square \square \square$ \\
\hline$\square$ & 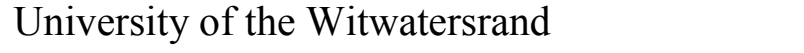 & $\square \square\|\| \square \square \square$ \\
\hline$\square$ & 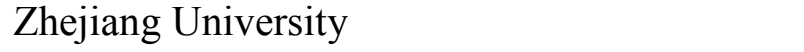 & $\square \square \| \square \square$ \\
\hline$\square \square$ & 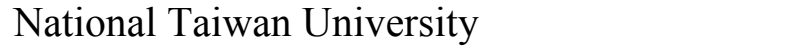 & $\square \square \| \square$ \\
\hline
\end{tabular}

While South Africa's tertiary education is also characterized by dualistic outcomes because of

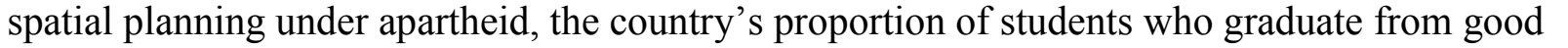
प

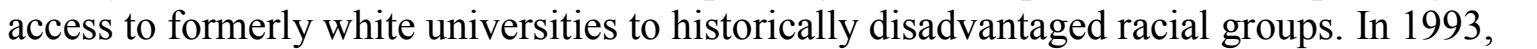

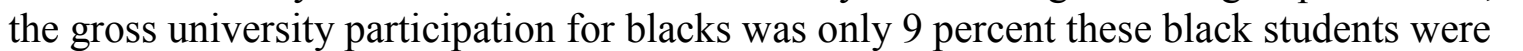

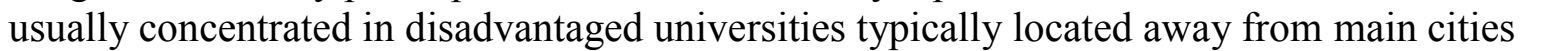
प

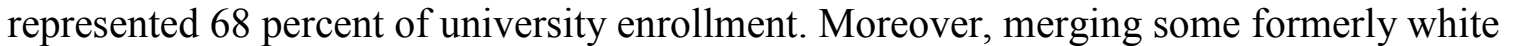

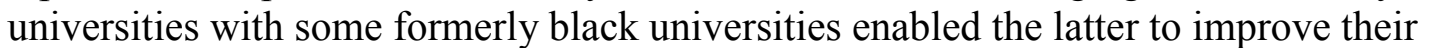

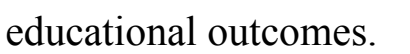

$\square$

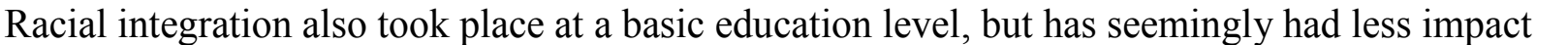

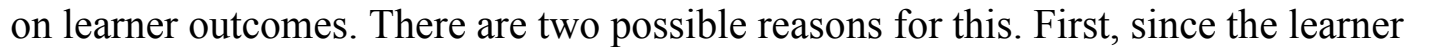

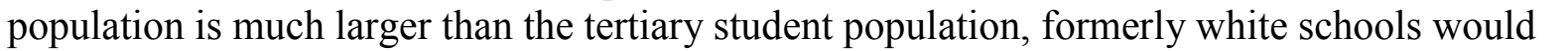

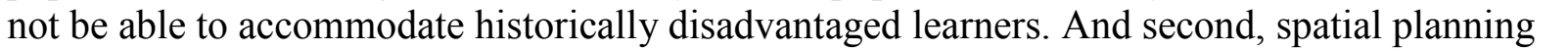

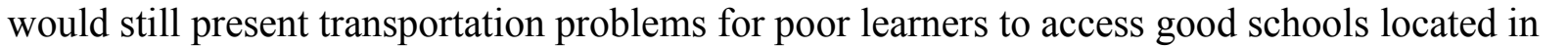

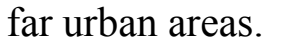




\section{KEY EXPLANATORY FACTORS}

Multiple and complex factors contribute to South Africa's underperformance in $\square \square \square \square\|ा\| \square$

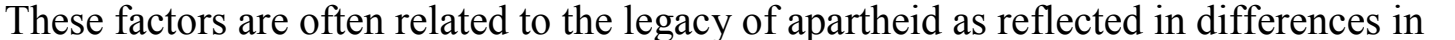

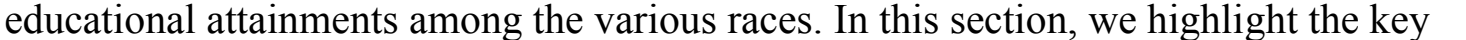

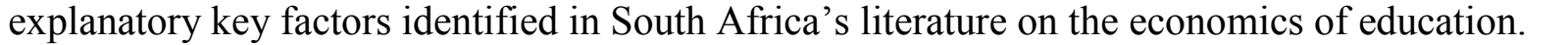
$\square$

\section{A. Overview of Salient Factors}

\section{Funding}

South Africa's expenditure on education is characterized by substantial disparities between

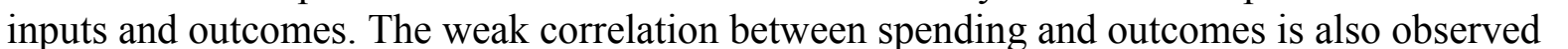

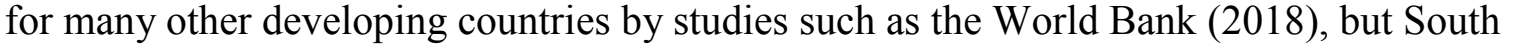

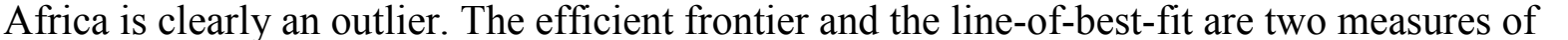

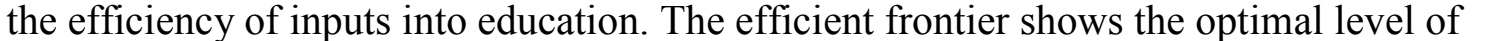

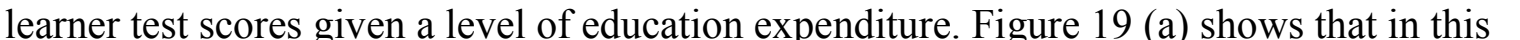

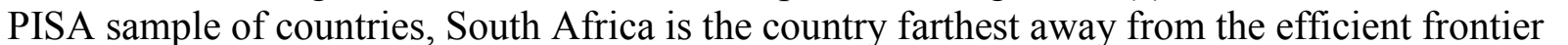

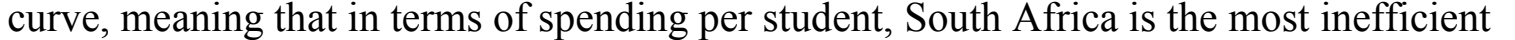

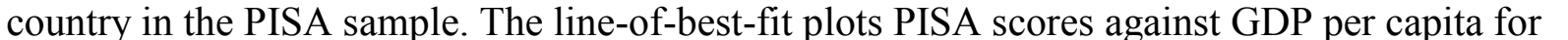

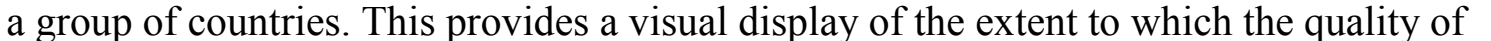

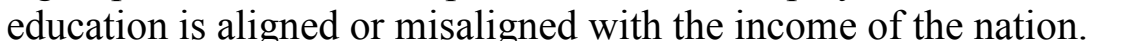

$\square$

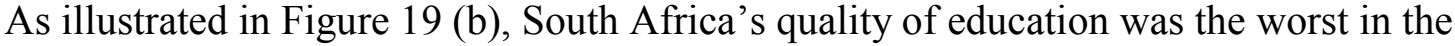

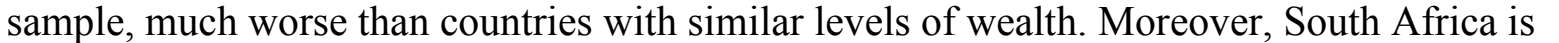

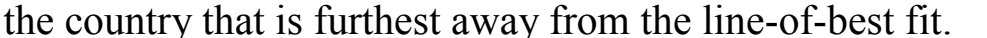

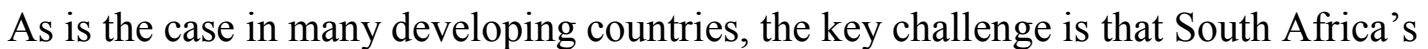

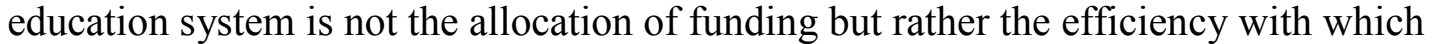

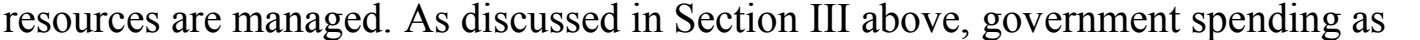

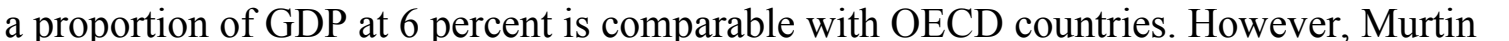

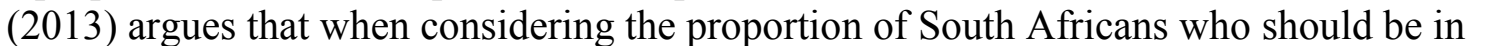

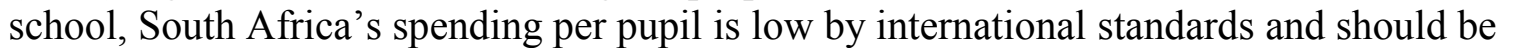
पा

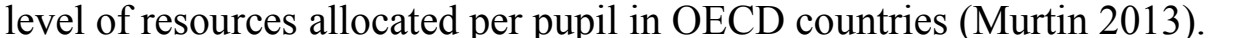

$\square$

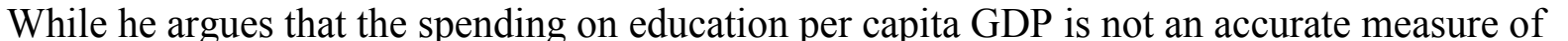

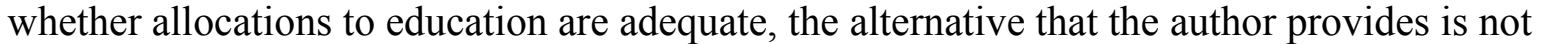

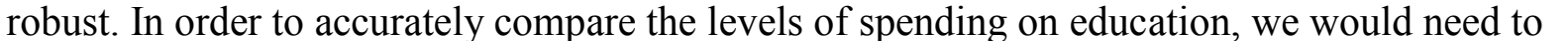

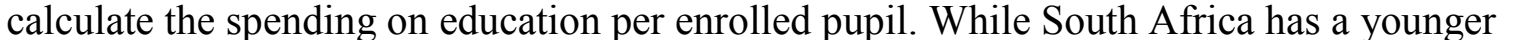
population than many OECD countries, many of South Africa's young people, particularly

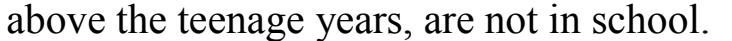



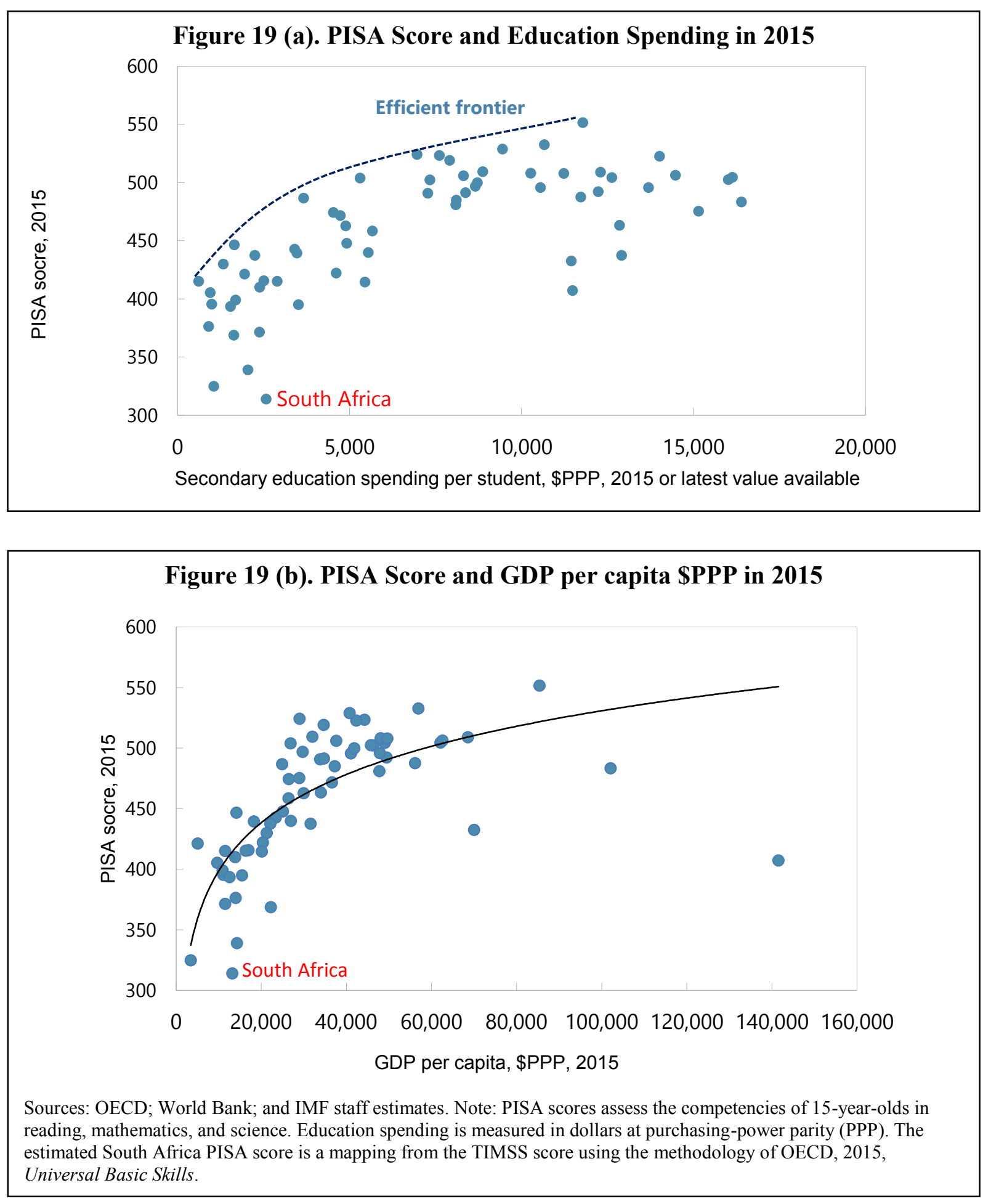

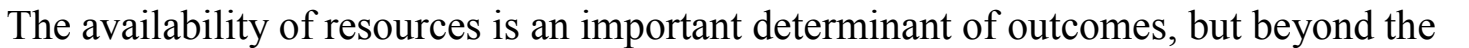

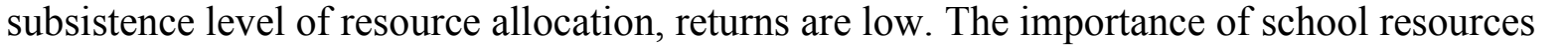

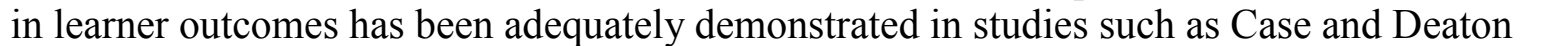

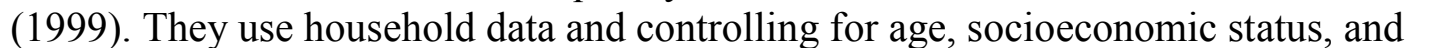

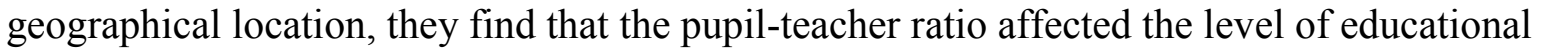

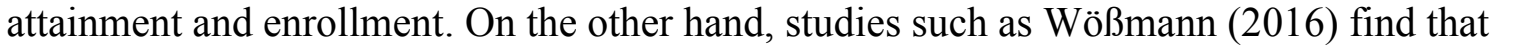




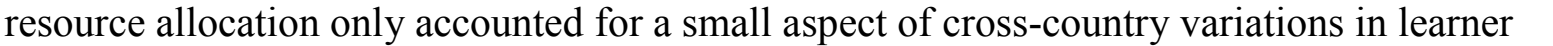

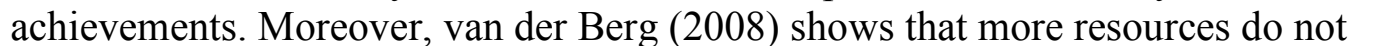

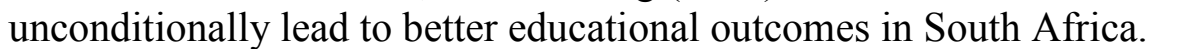

$\square$

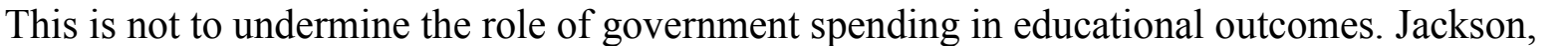

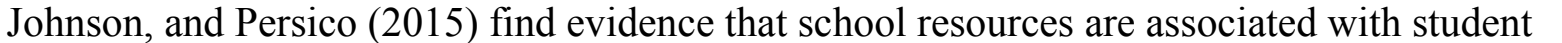

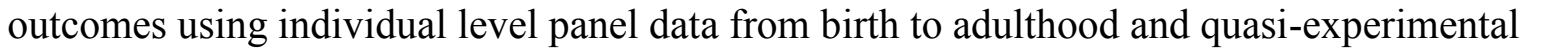

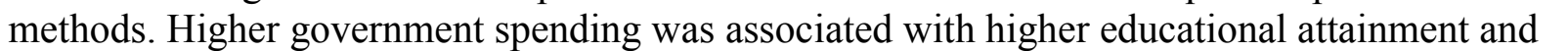

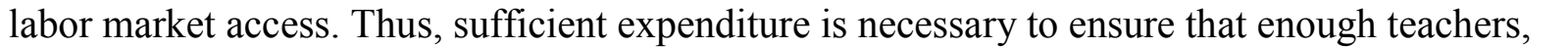

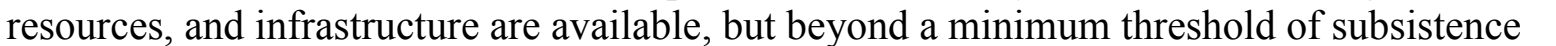

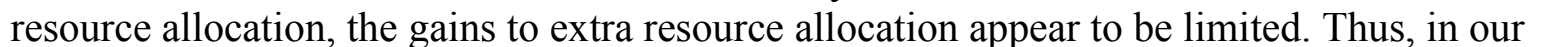

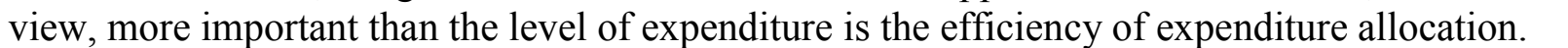

\section{Teacher subject content knowledge}

$\square$

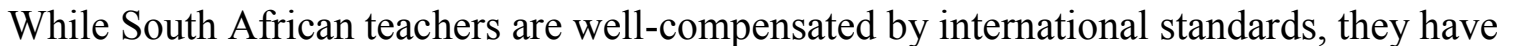
ए

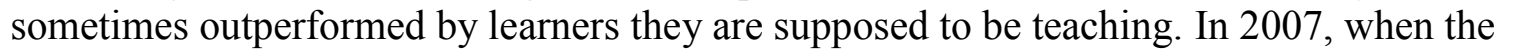

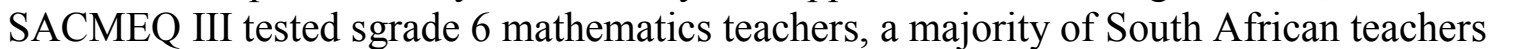

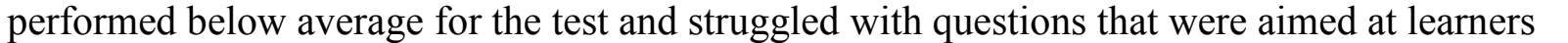

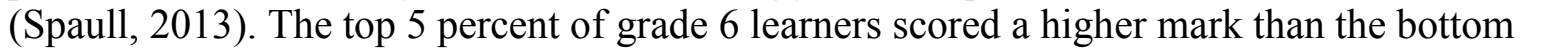

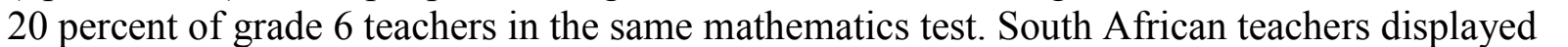

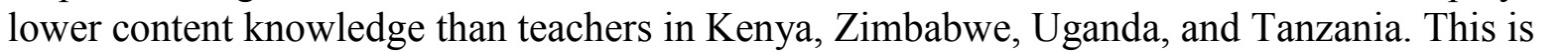

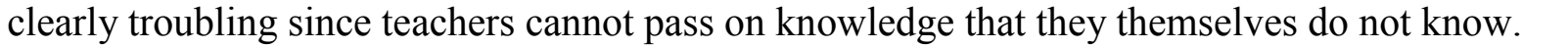
$\square$

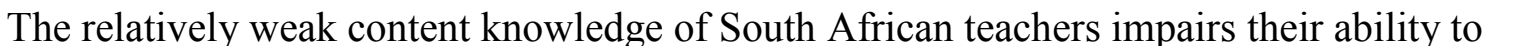
accurately judge their learners' performance and in turn their ability to improve learner

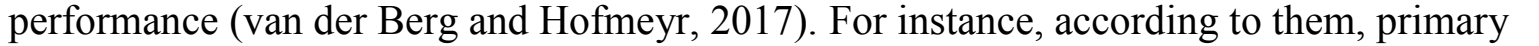

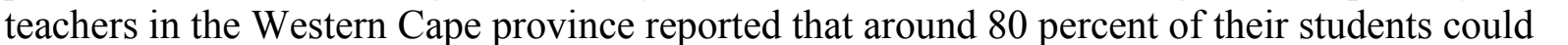

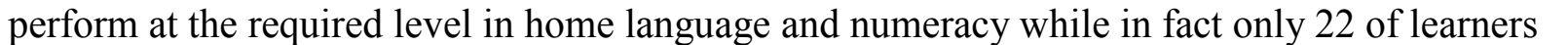

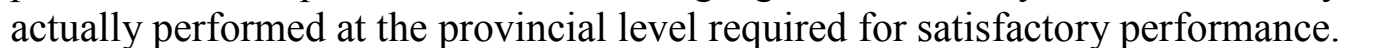

\section{$\square$}

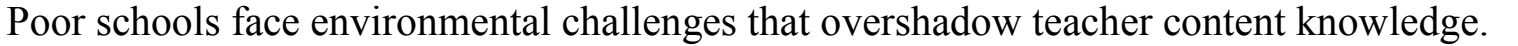

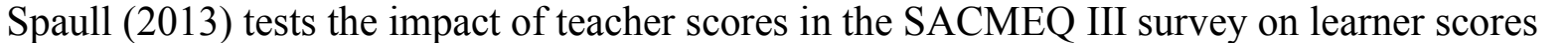

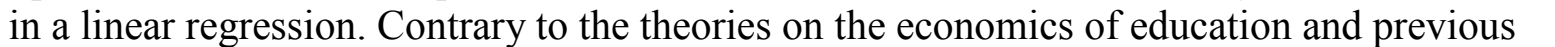

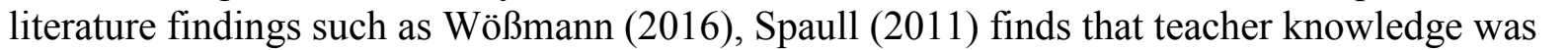

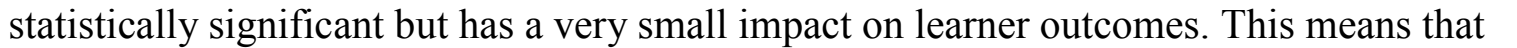

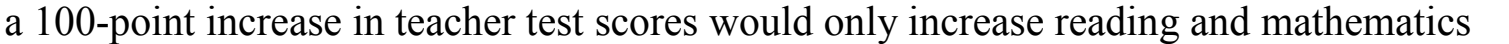

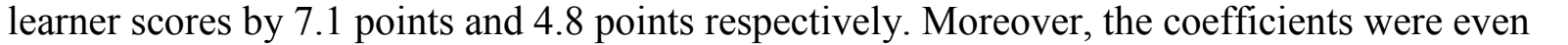

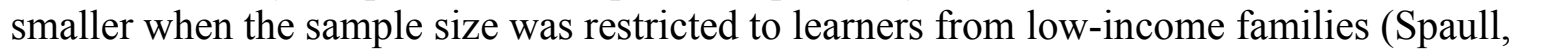

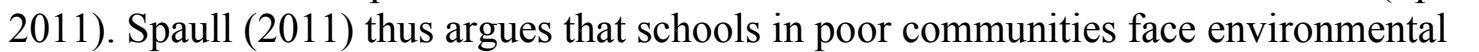

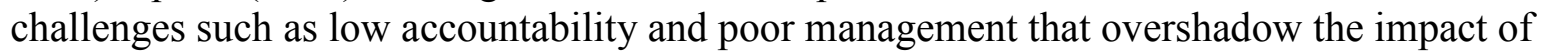

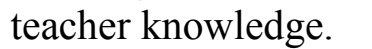

(1)

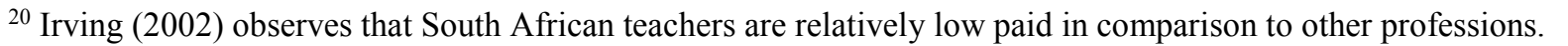

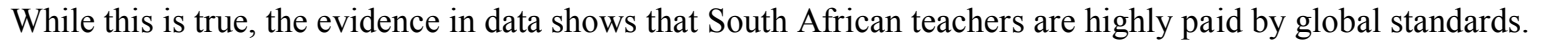




\section{Teacher accountability, motivation and absenteeism $\square$}

$\square$

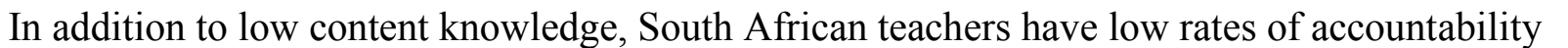

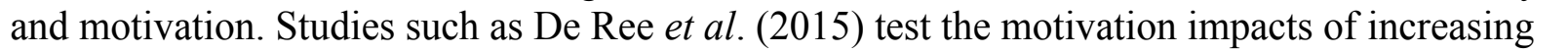

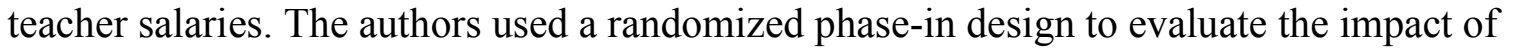

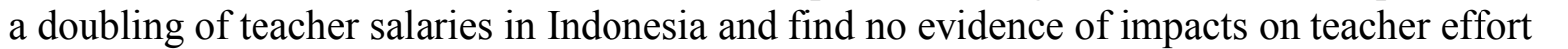

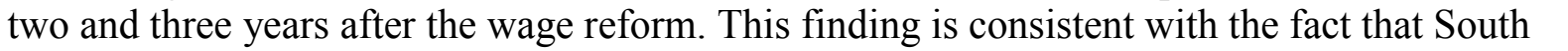

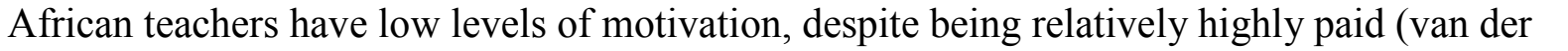
$\square \square \|$ et al $\square\|\|\|\|$

$\square$

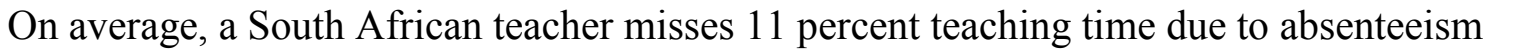

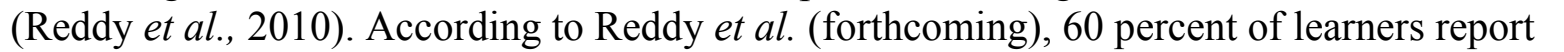

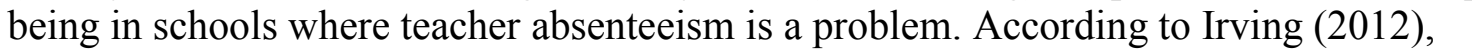

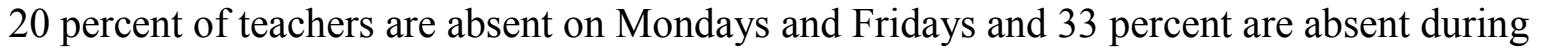

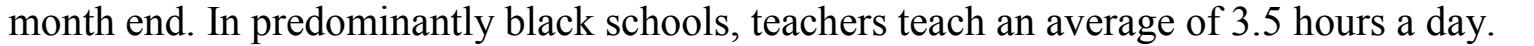

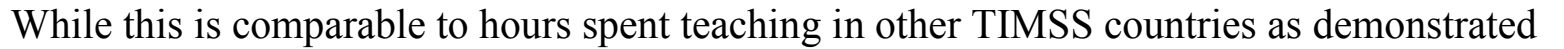

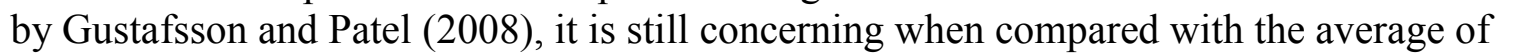

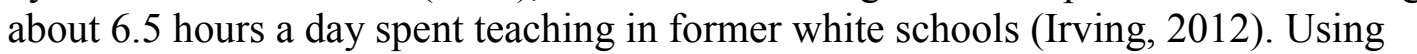

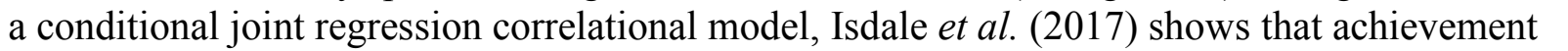

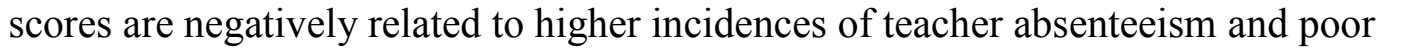

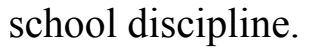

$\square$

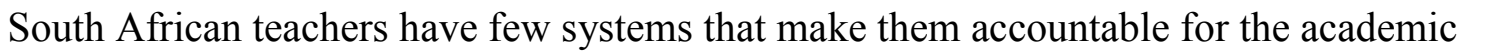

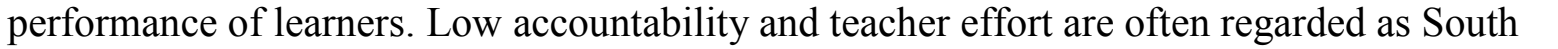

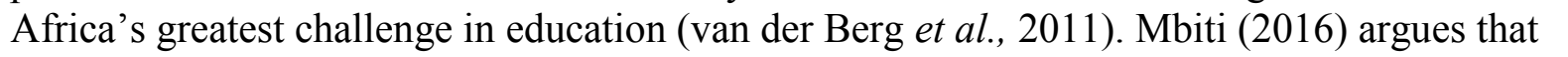

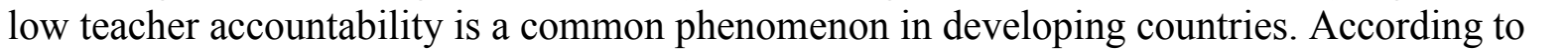

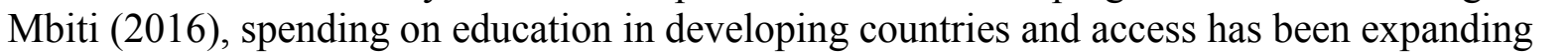

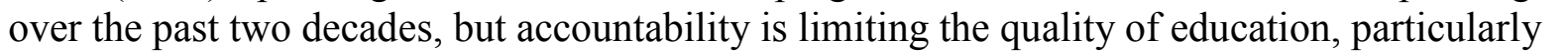

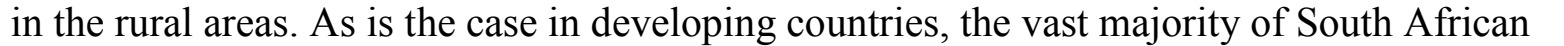

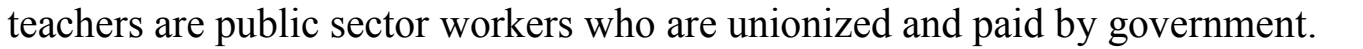

$\square$

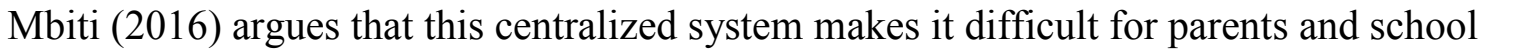

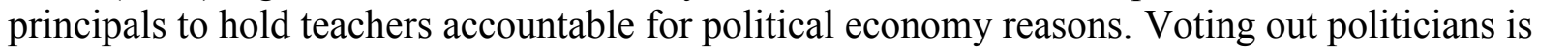

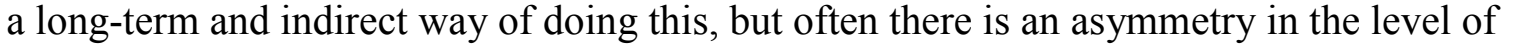

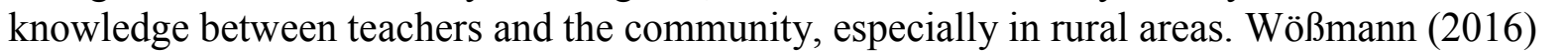

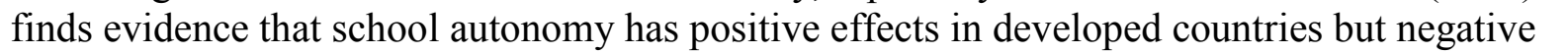

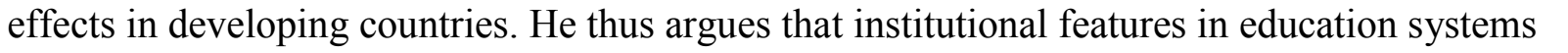

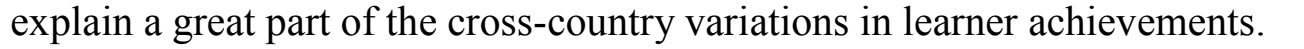

$\square$

\section{The teaching profession}

$\square$

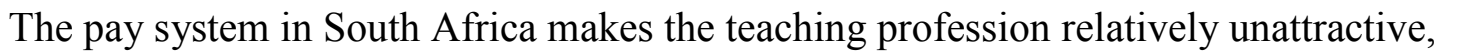

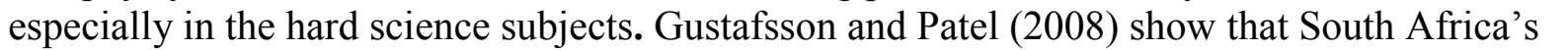

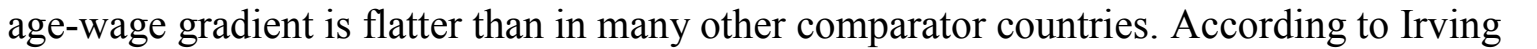

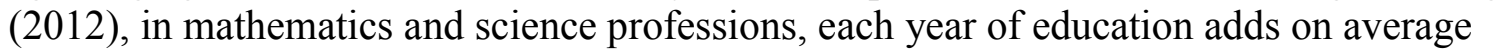

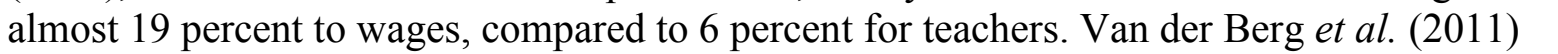

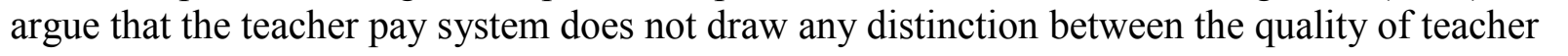




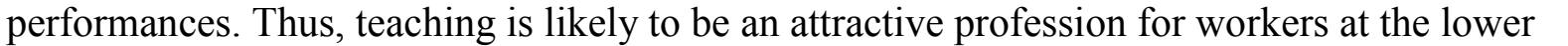

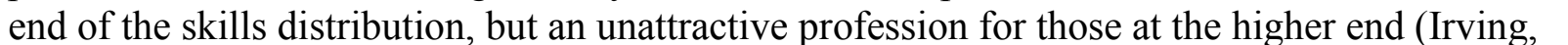

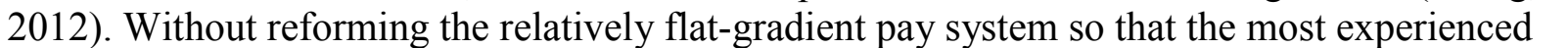

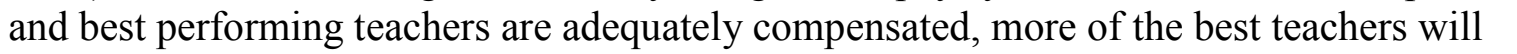

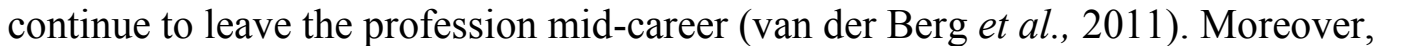
ए

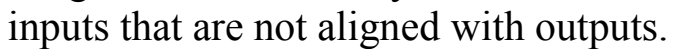

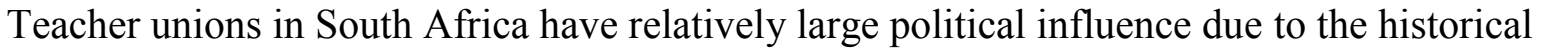

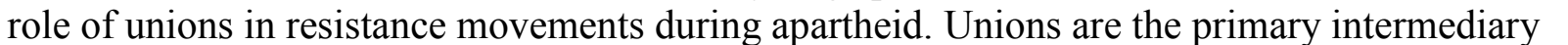

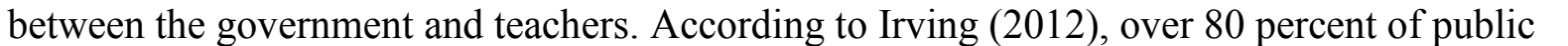

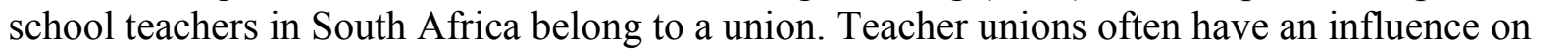

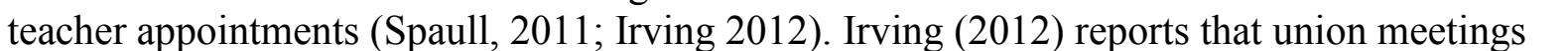

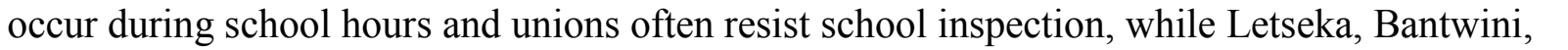

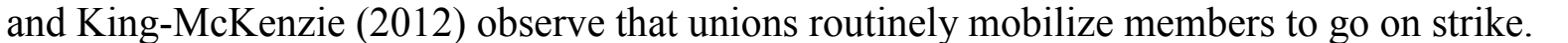

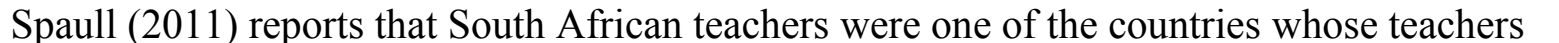

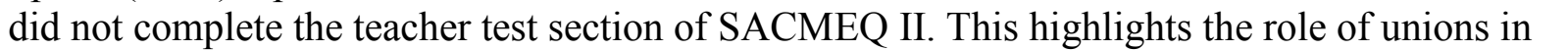

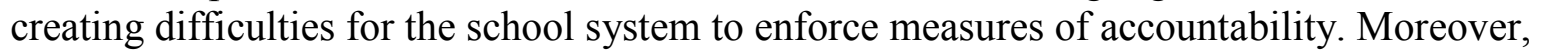

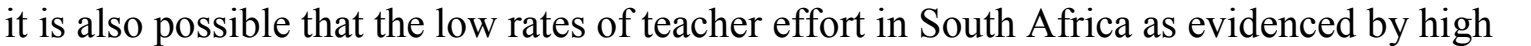

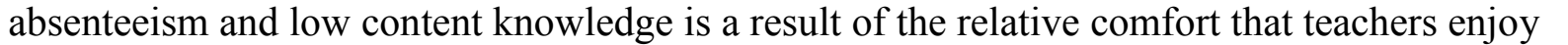

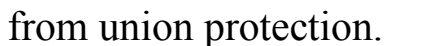

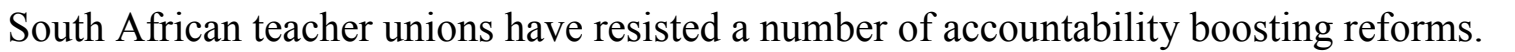

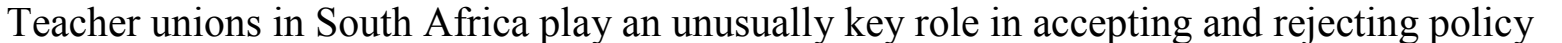
reforms (van der Berg and Hofmeyr, 2017). The scope of South Africa's teacher unions

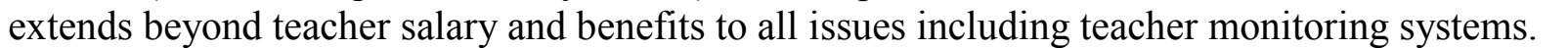

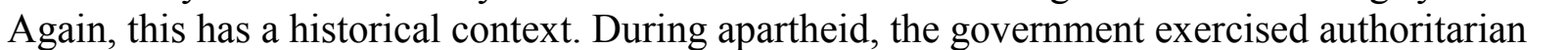

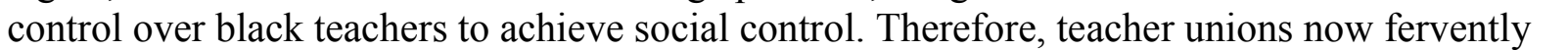

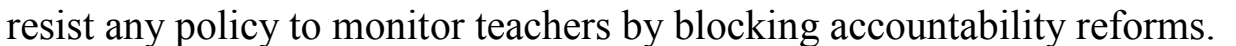

\section{School management}

$\square$

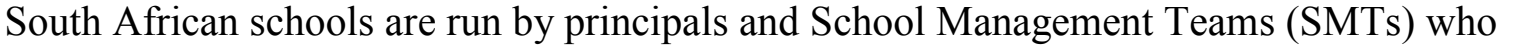

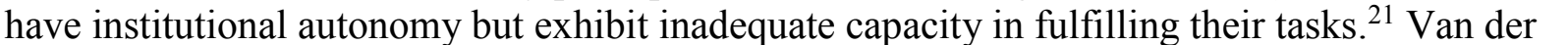

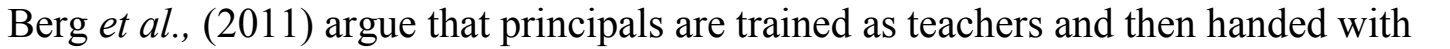

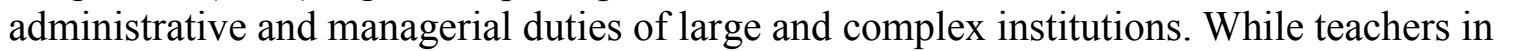

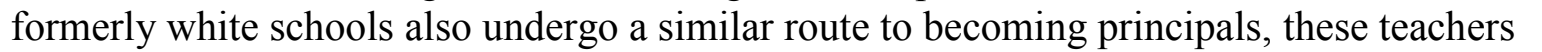

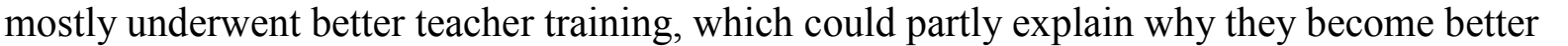

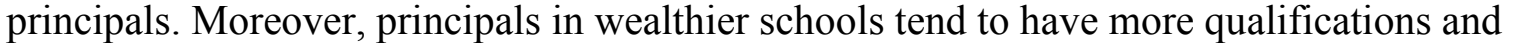

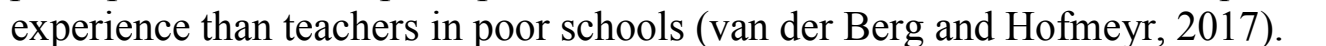
$\square$

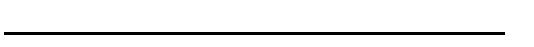

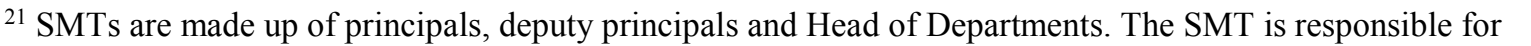

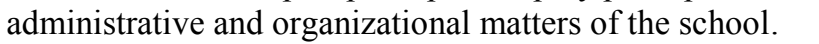




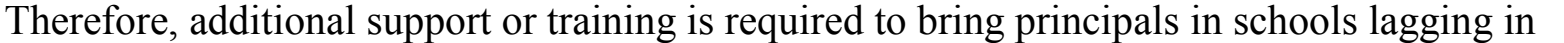

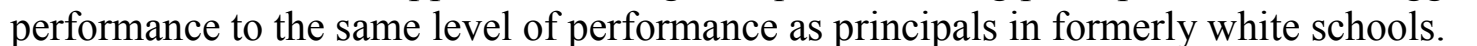

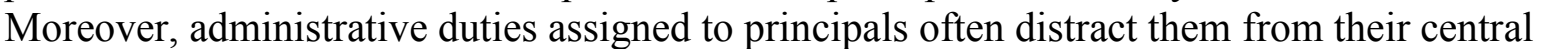

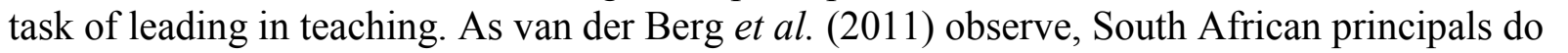

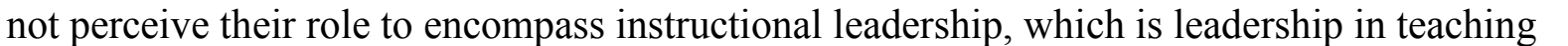

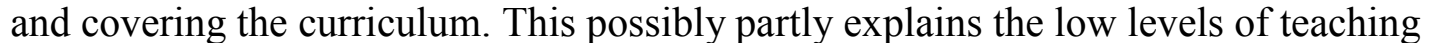

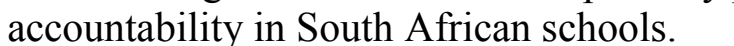

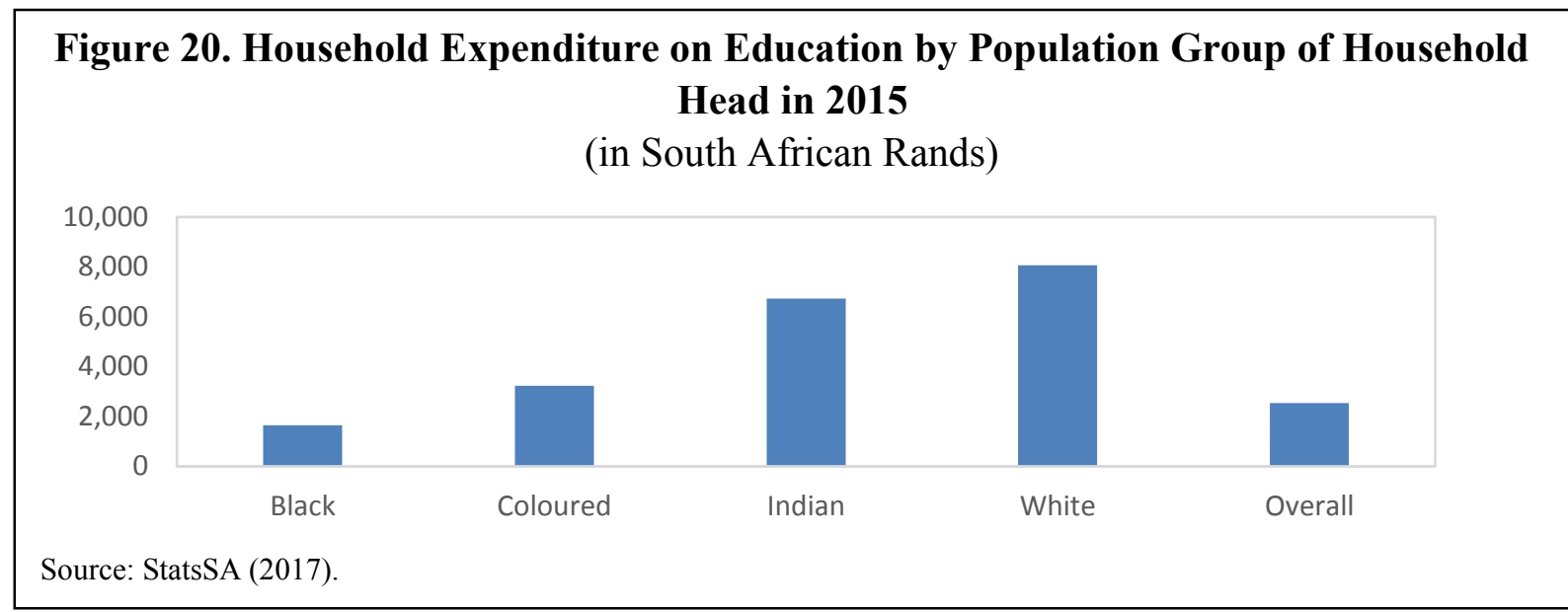

$\square$

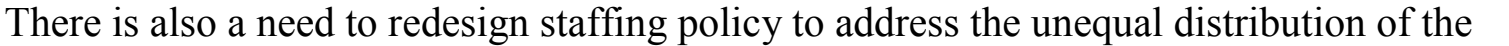

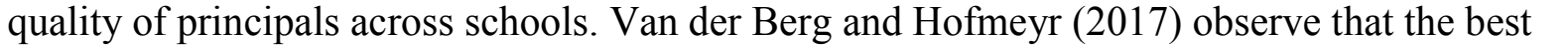

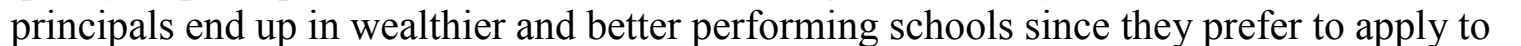

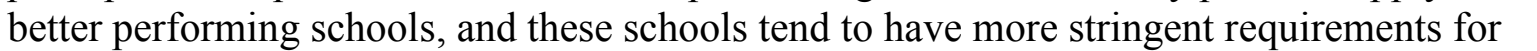

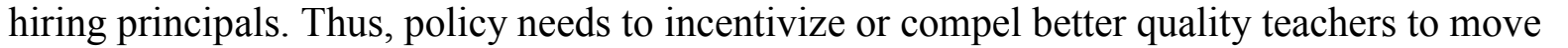

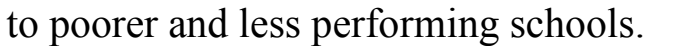

$\square$

Income inequality, socio-economic status, and geographical location

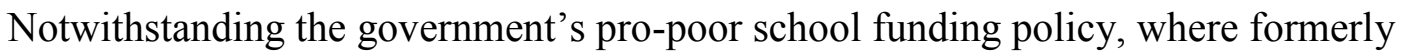

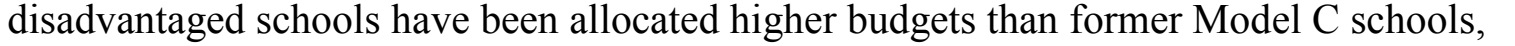

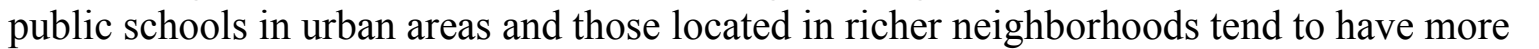

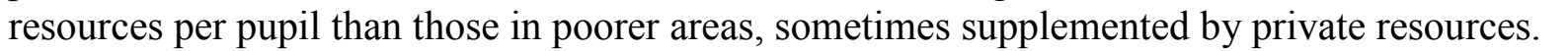

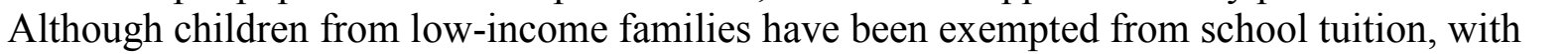

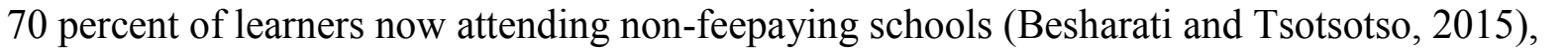

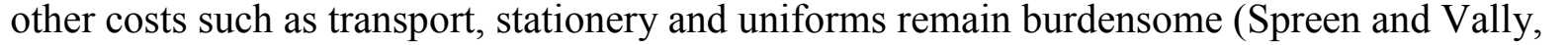

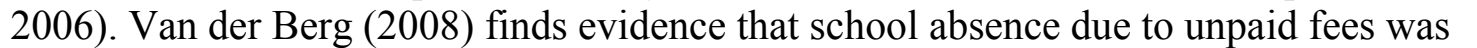

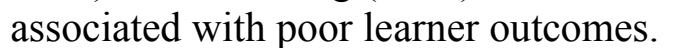

$\square$

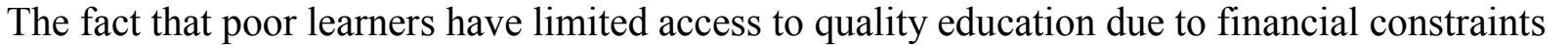
ए

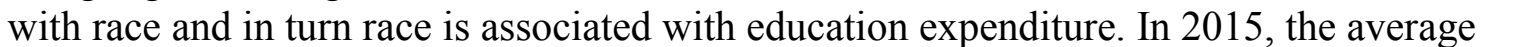

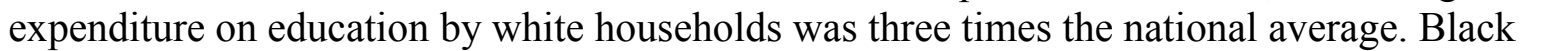

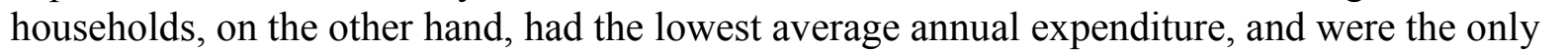

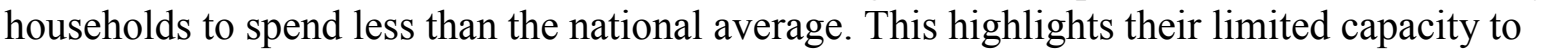

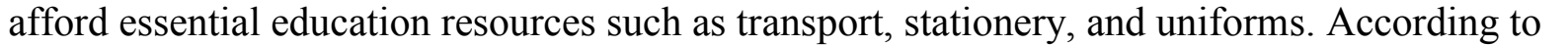

CInternational Monetary Fund. Not for Redistribution 


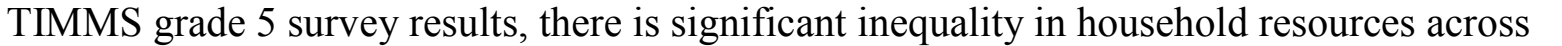

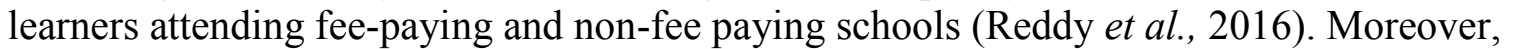

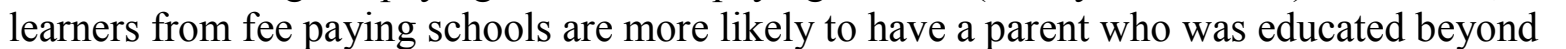

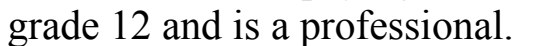

$\square$

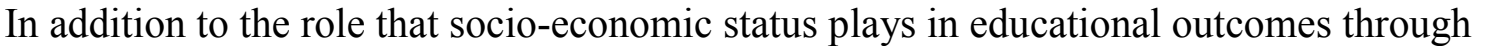

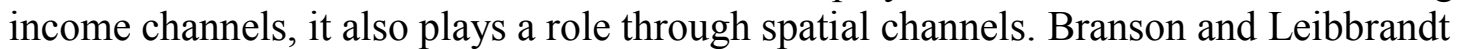

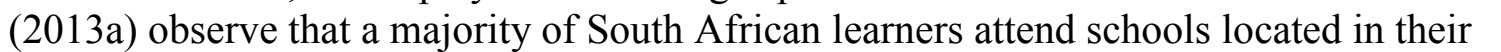

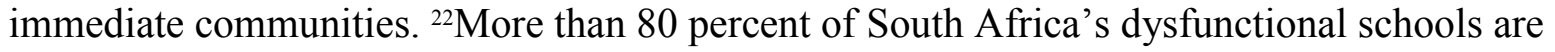

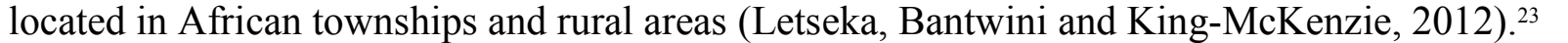

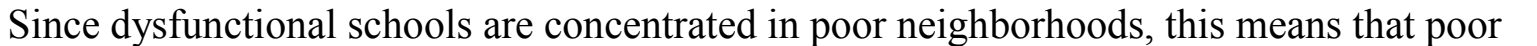

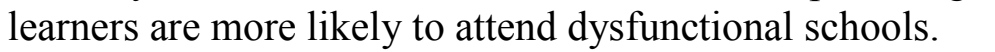

$\square$

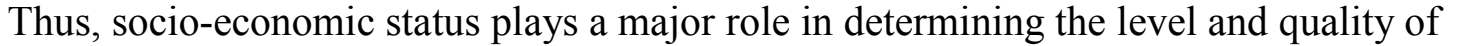

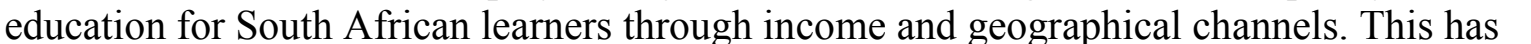

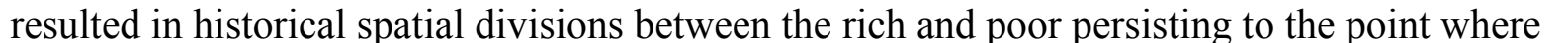

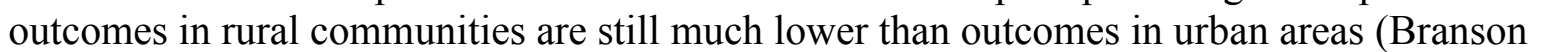

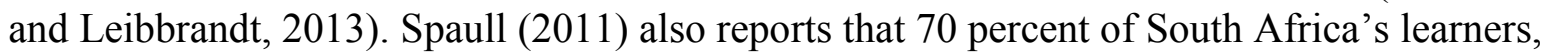

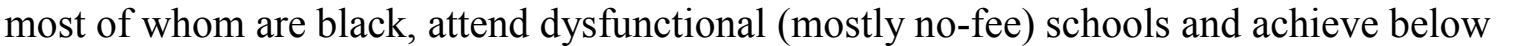

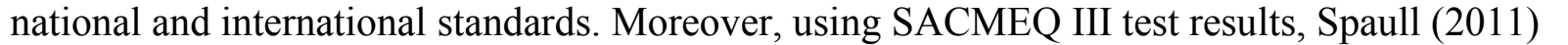
ए $\square$ ए।

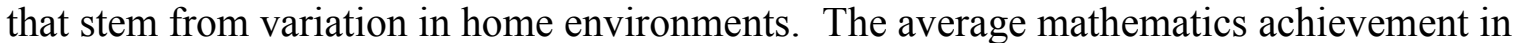

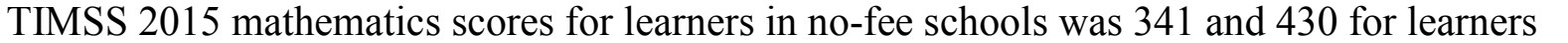

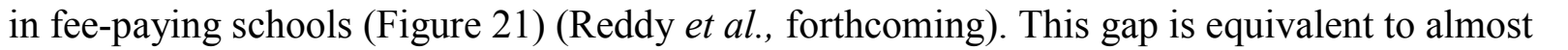

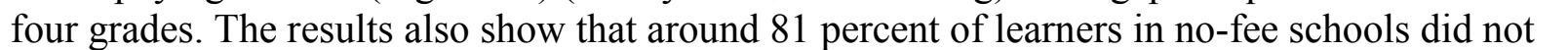

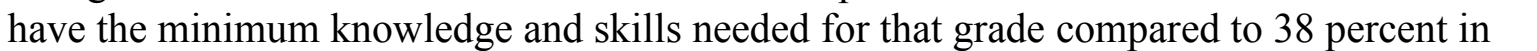

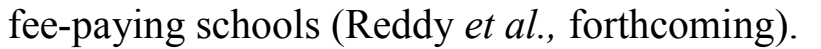

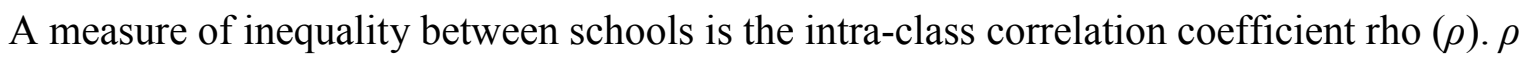

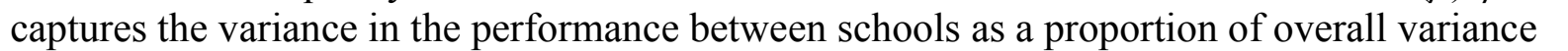

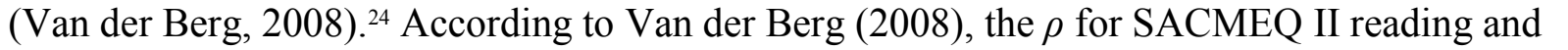

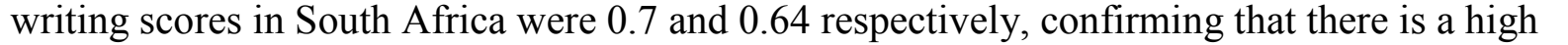

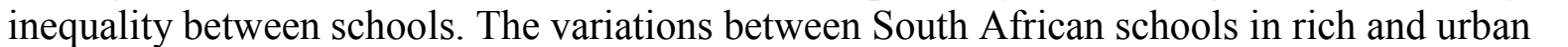

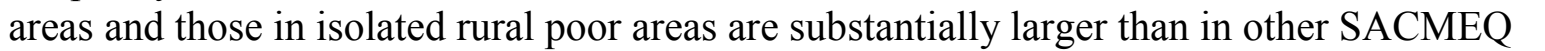

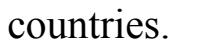

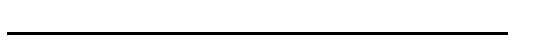

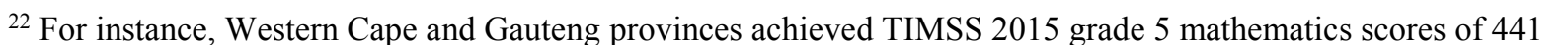

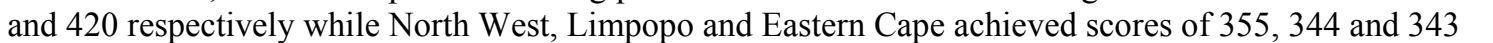

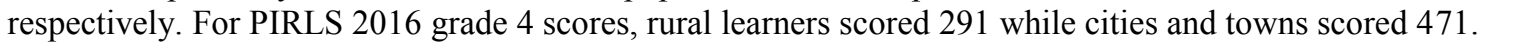

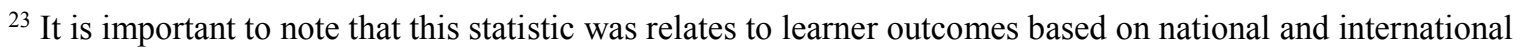

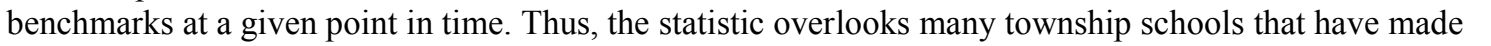

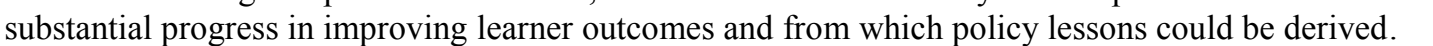

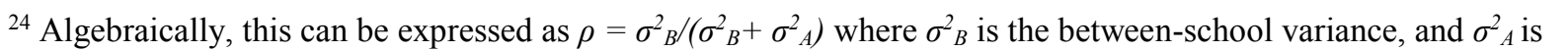

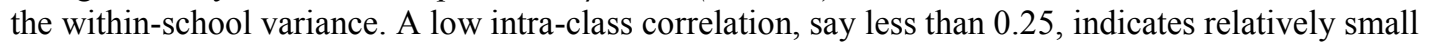

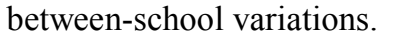


Figure 21. Average TIMSS 2015 Mathematics Score and Competence level by non-fee and fee-paying schools

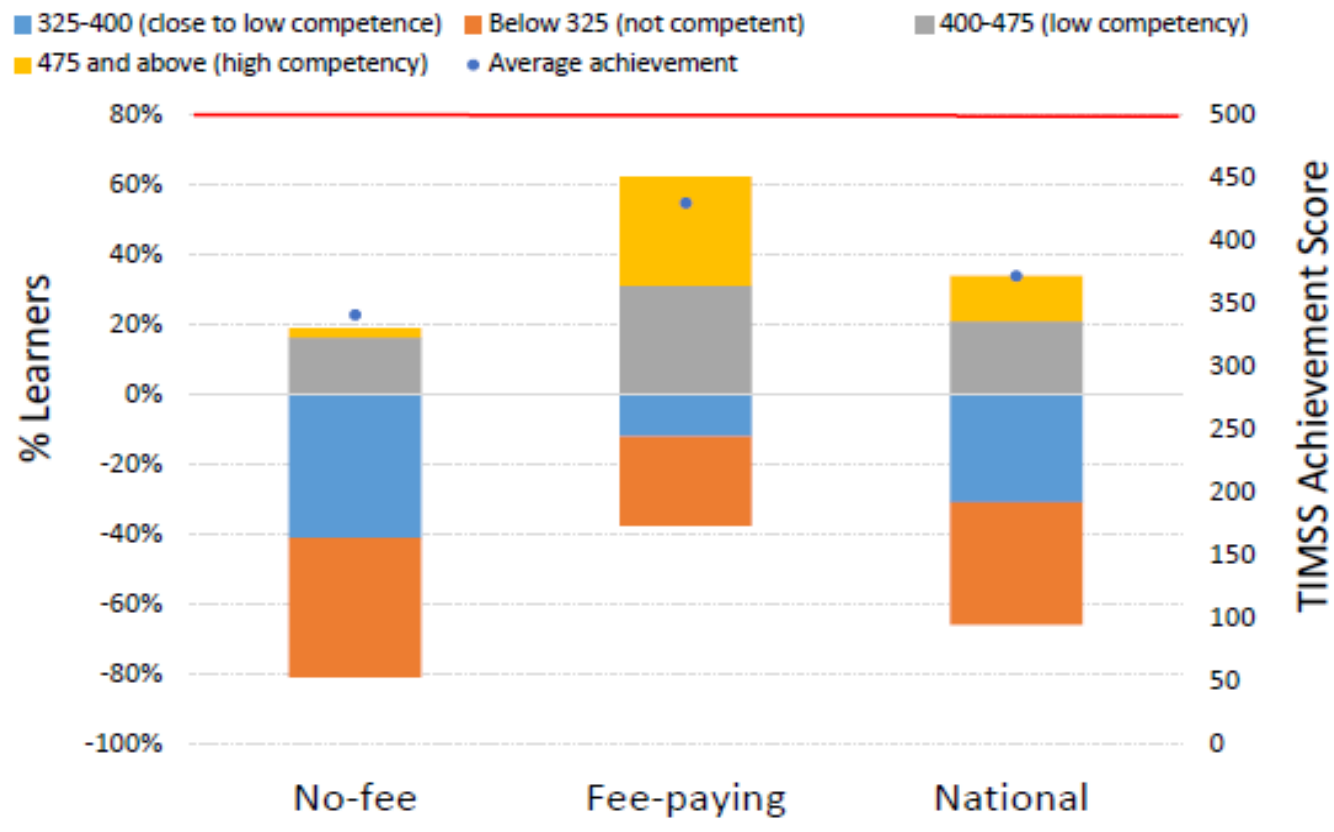

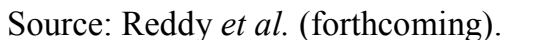

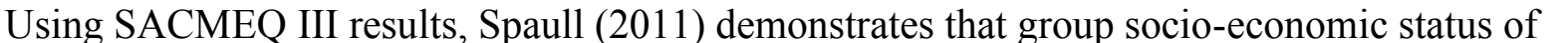

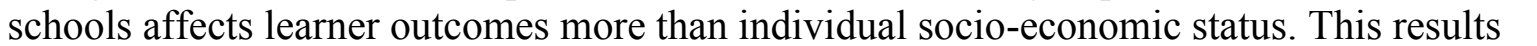

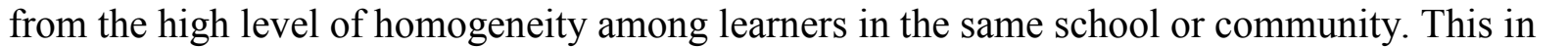

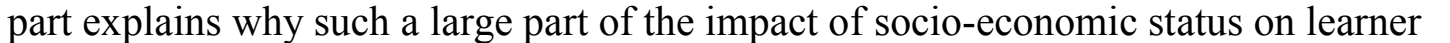

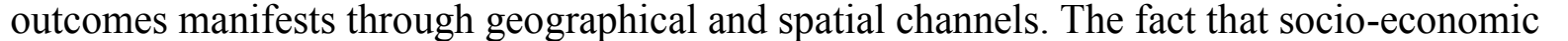

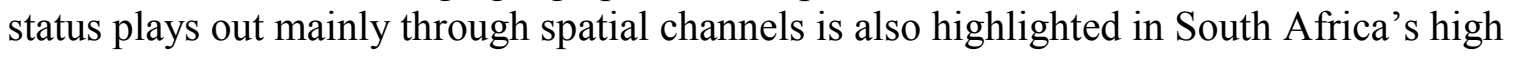

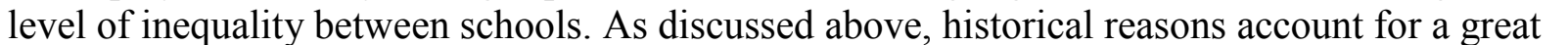

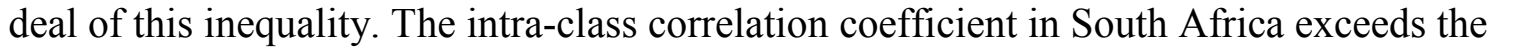

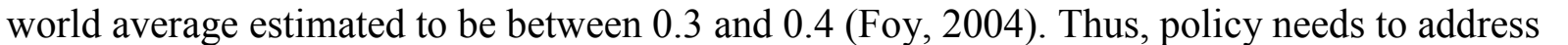

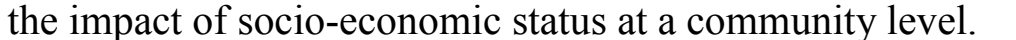

$\square$

\section{Language}

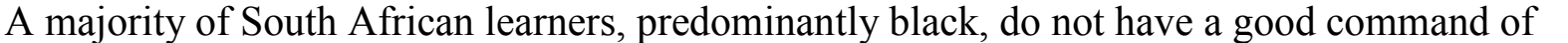

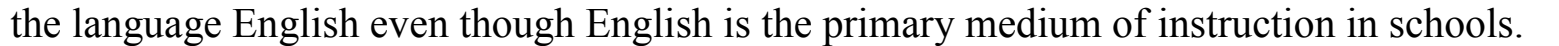

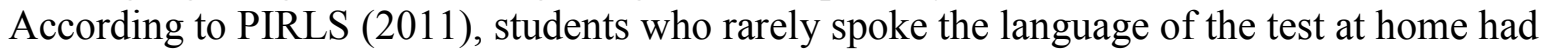

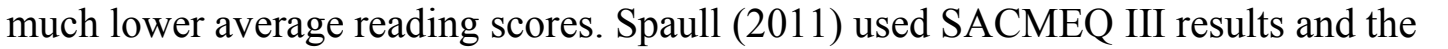

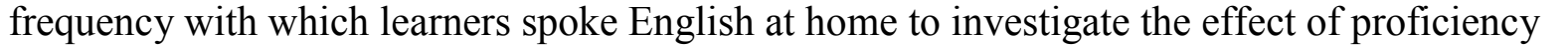

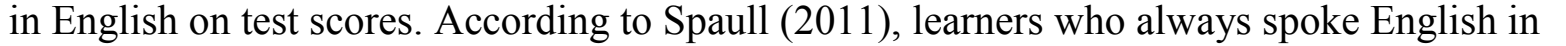
ए

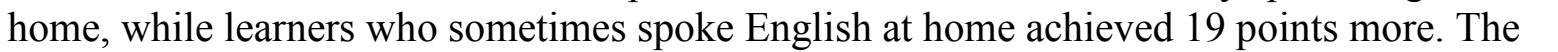

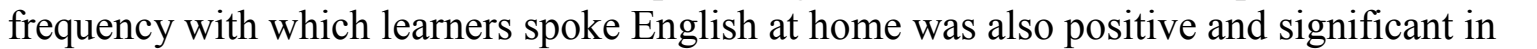

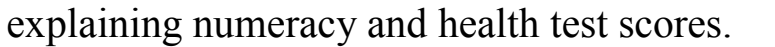

$\square$ 


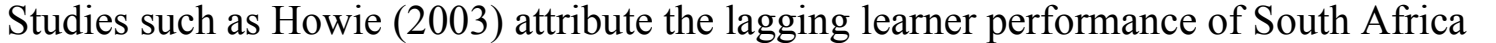

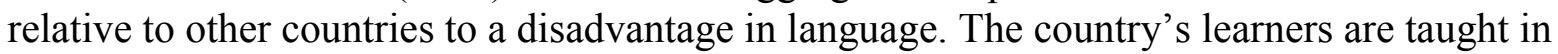

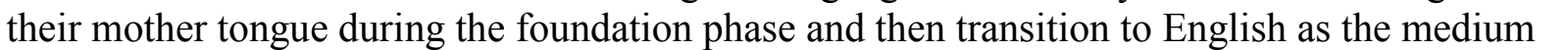

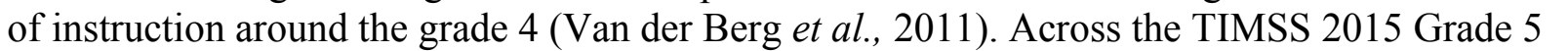

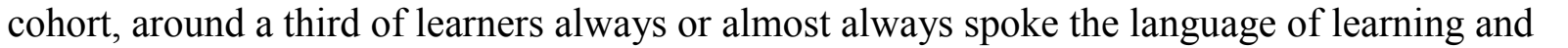

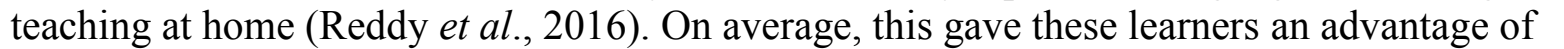

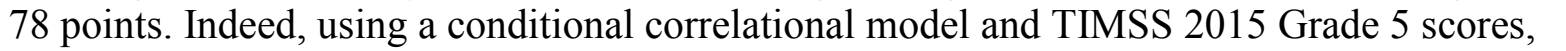

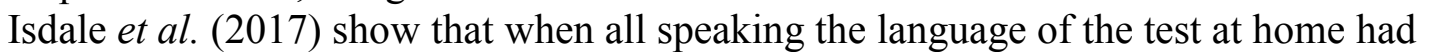

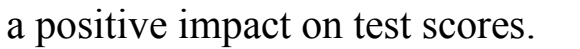

$\square$

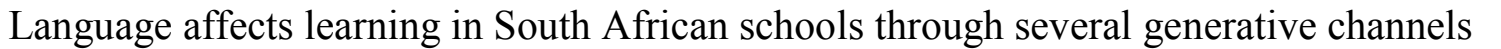

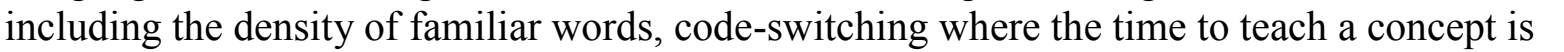

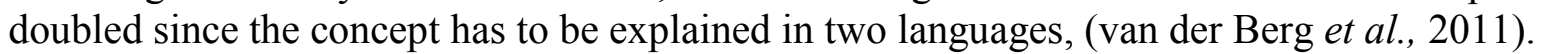

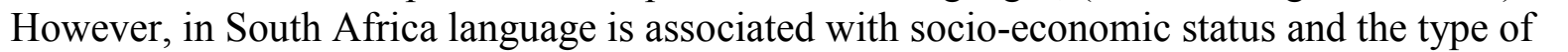

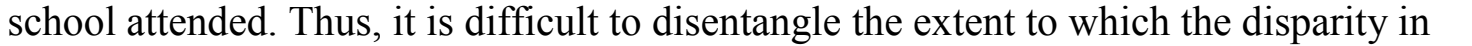

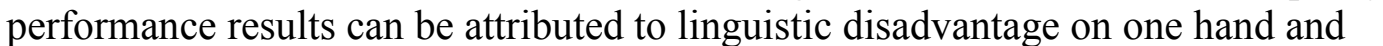

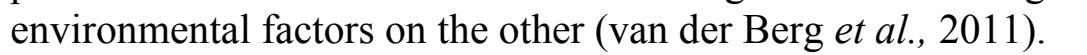
$\square$

\section{Textbooks and other resources}

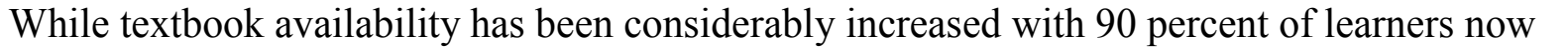

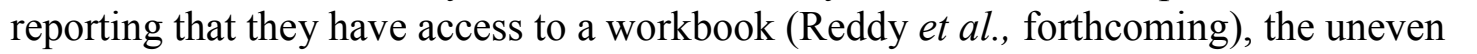

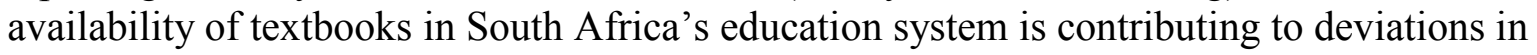

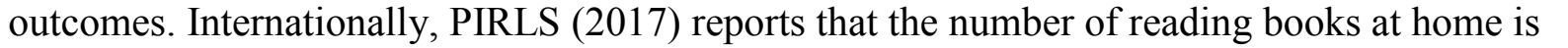

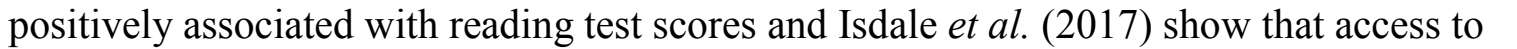

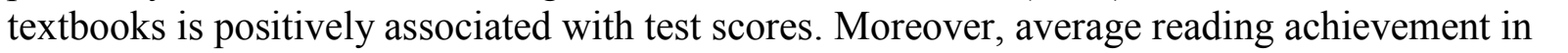

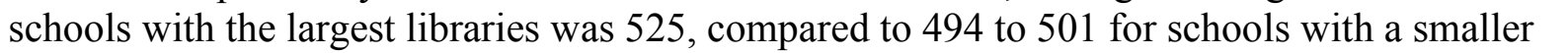

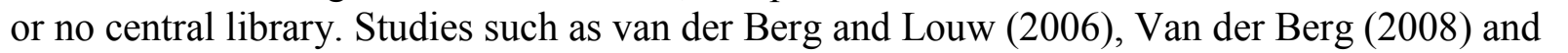

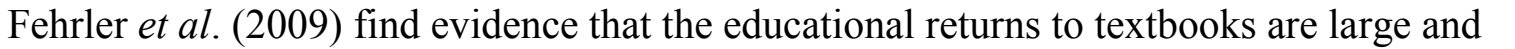
significant in South Africa's education production function.

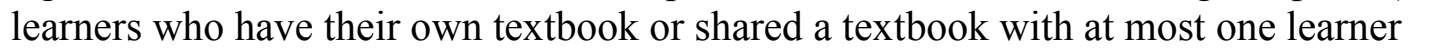

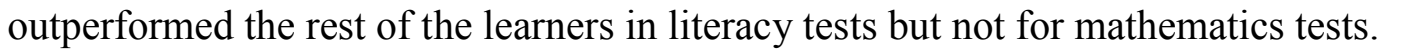
$\square$ एव

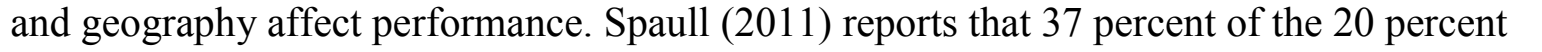

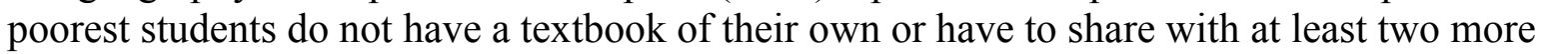

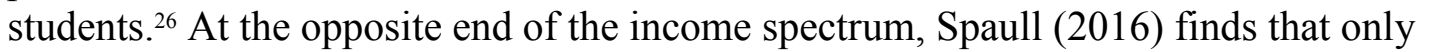

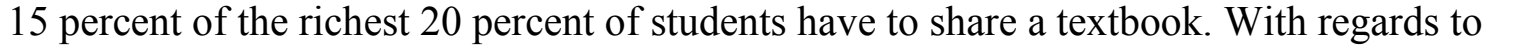

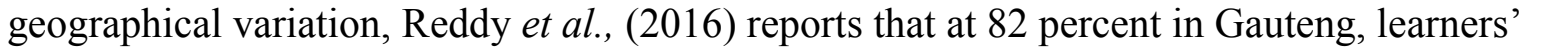

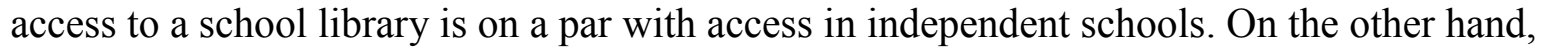

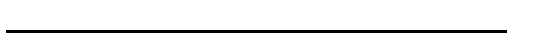

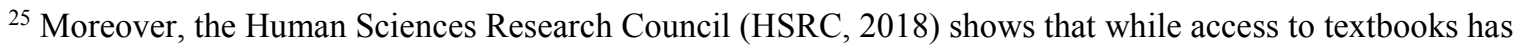
प

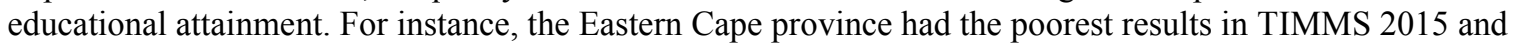

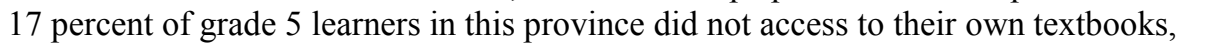

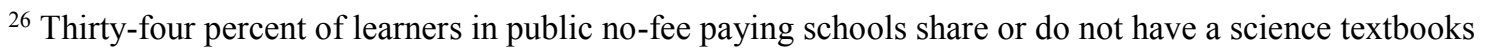

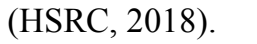




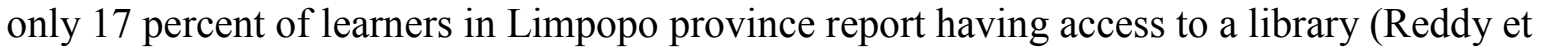

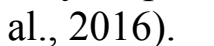

$\square$

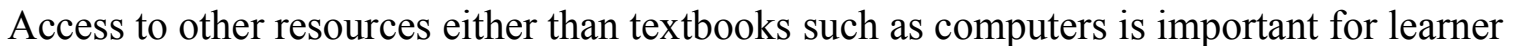

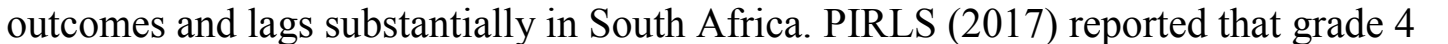

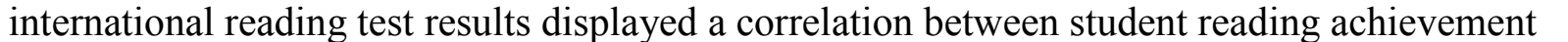

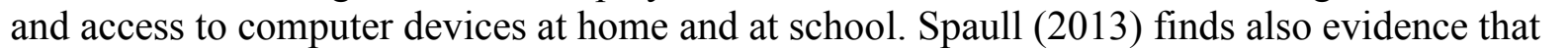

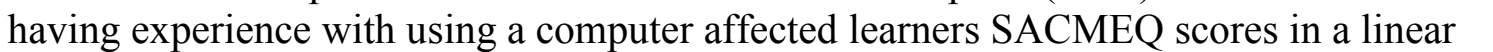

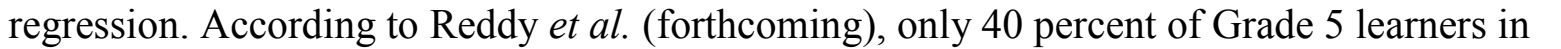

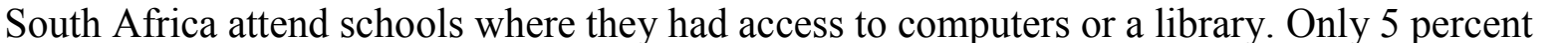

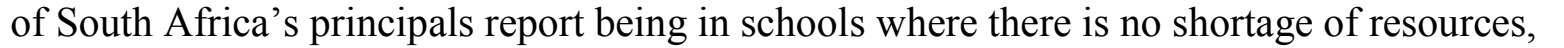

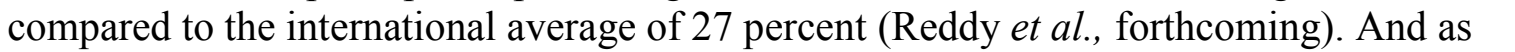

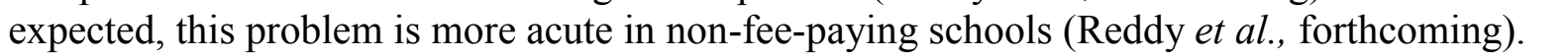
$\square$

\section{B. The Main Take-Aways}

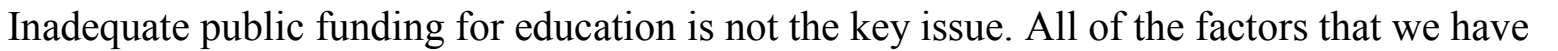

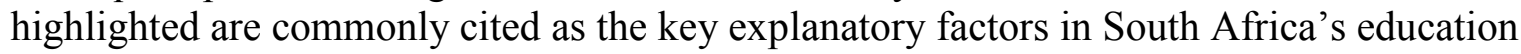
system. While Murtin (2013) argues that South Africa's primary and secondary education is

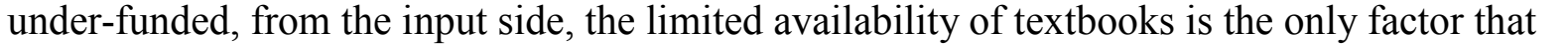

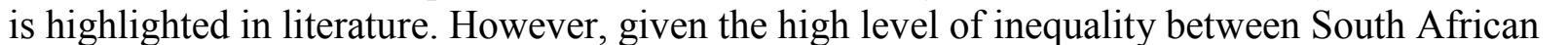

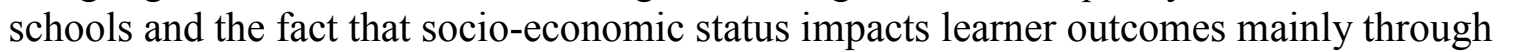

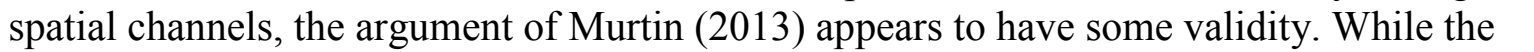
$\square\|\| \| \backslash$ level of South Africa's education expenditure is high by international standards,

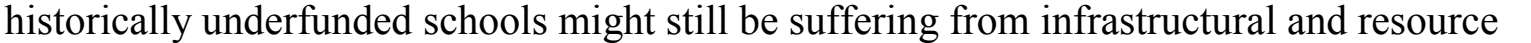

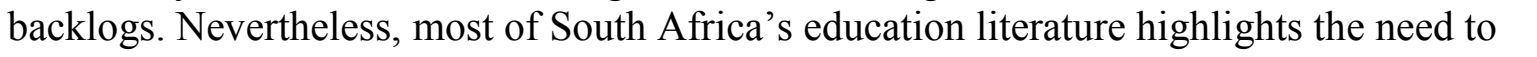

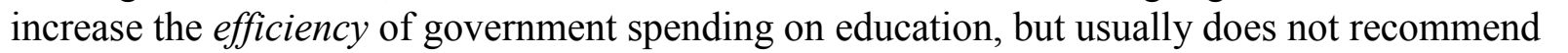

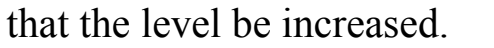

$\square$

The major beneficiaries of South Africa's high education expenditure are teachers despite

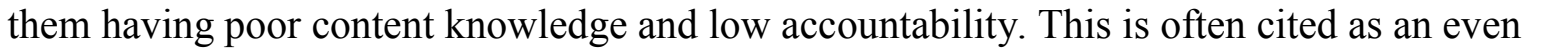

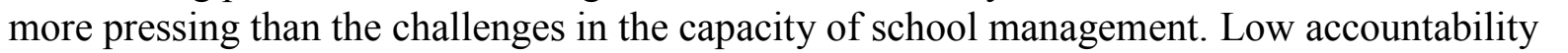

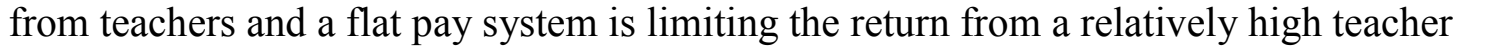

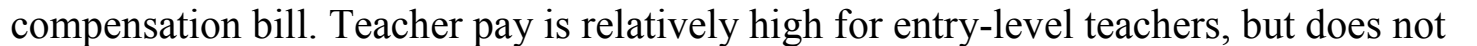

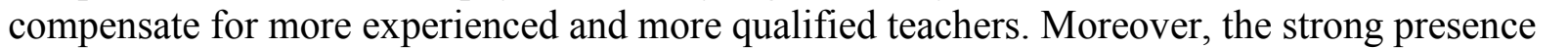

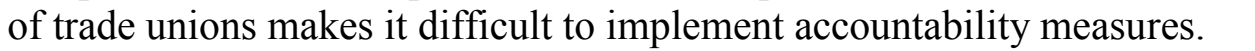

$\square$

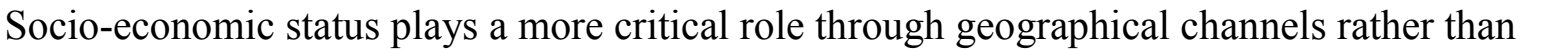

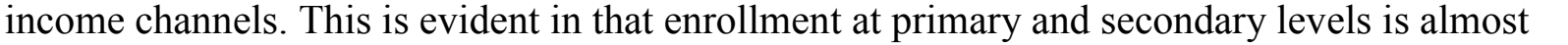

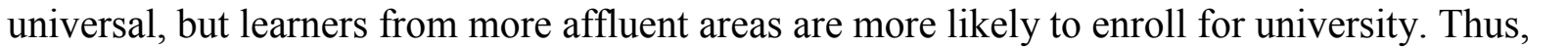

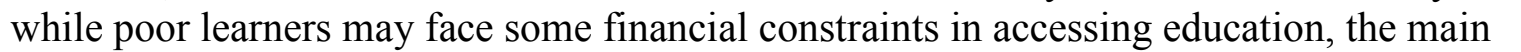

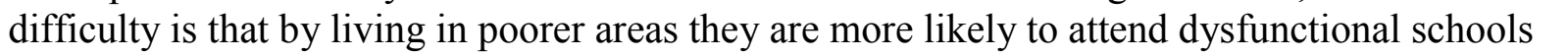
ए

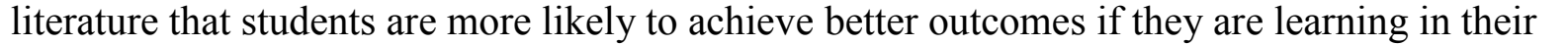

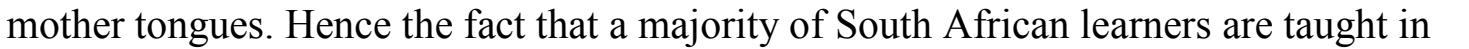

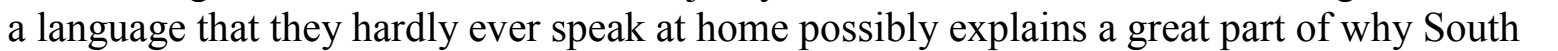

CInternational Monetary Fund. Not for Redistribution 


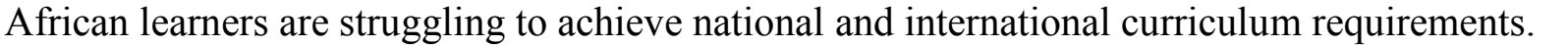

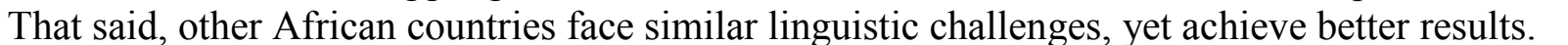
$\square$

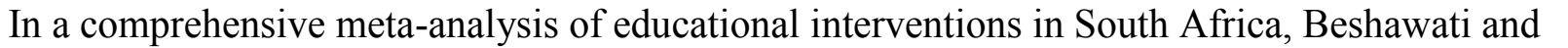

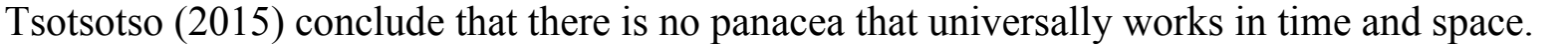

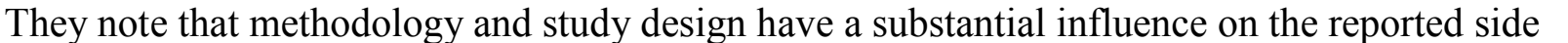
$\square\|\| \|$ of "treatment". The strongest results that they report are that, in line with child

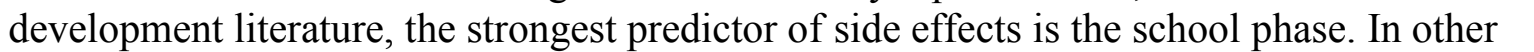

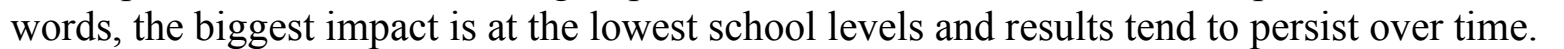

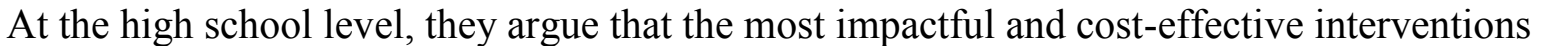

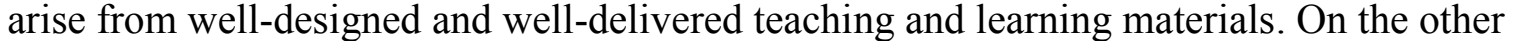

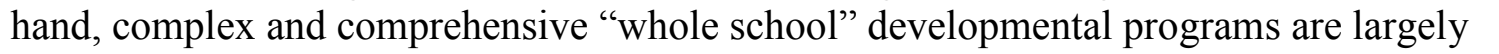

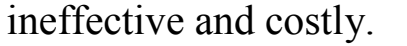

$\square$

\section{SOCIO-ECONOMIC IMPACTS OF WEAK EDUCATIONAL OUTCOMES}

\section{A. Economic Growth}

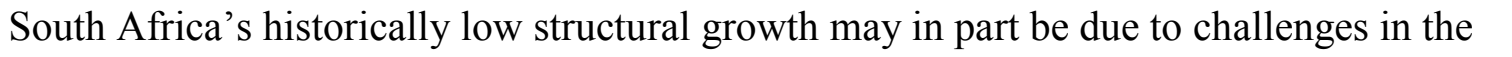

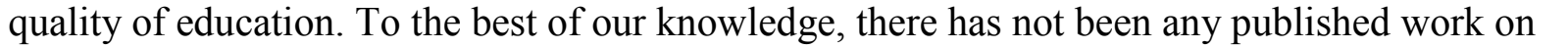

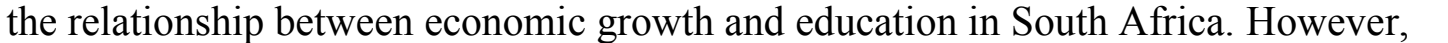

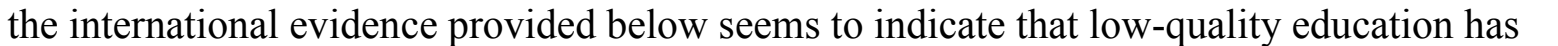

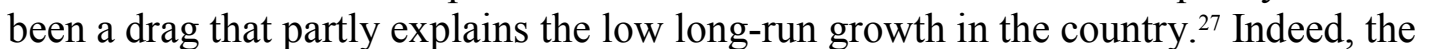

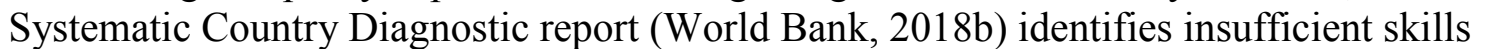

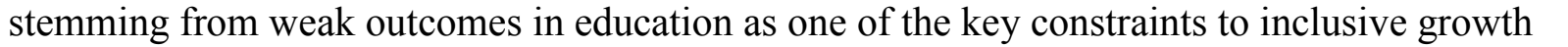

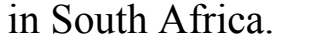

$\square$

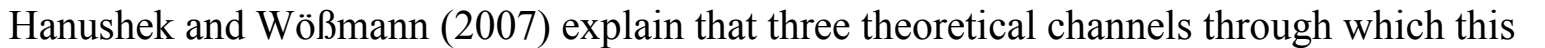

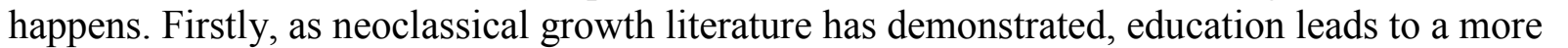

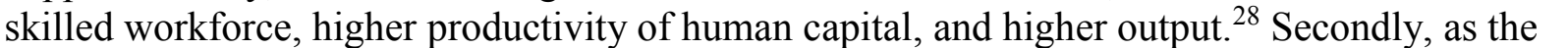

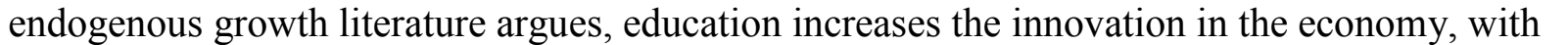

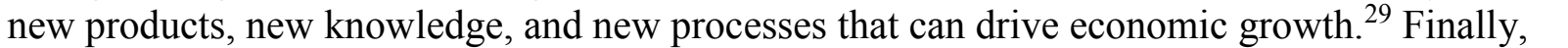

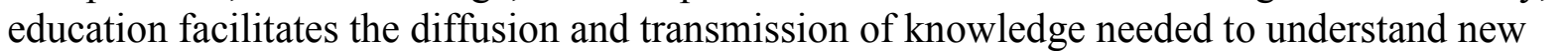

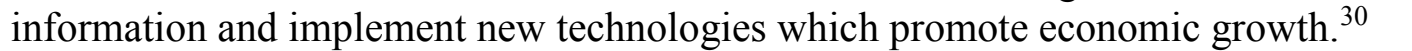
$\square$

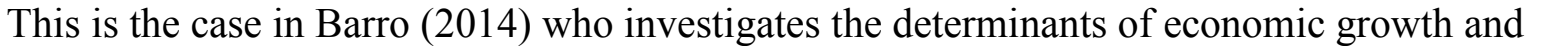
ए

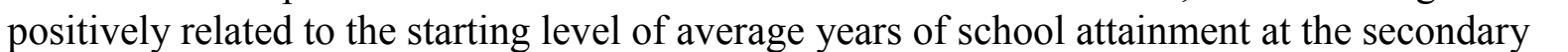

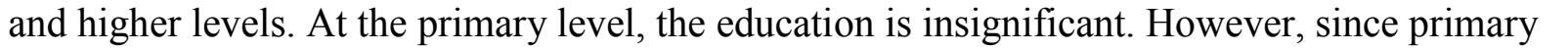

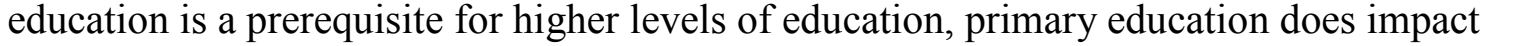

mmmmmmmmmmmmmm

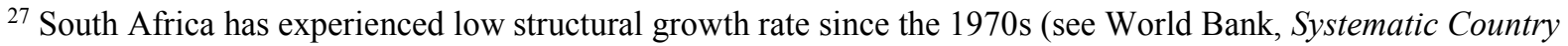

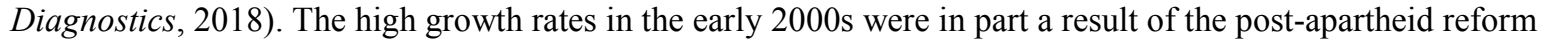

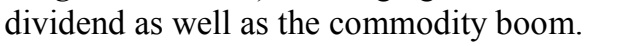

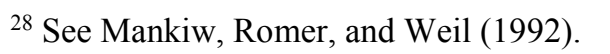

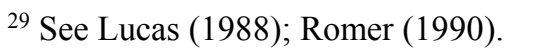

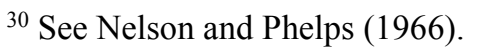




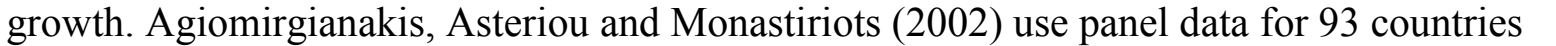

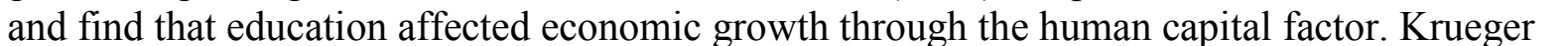

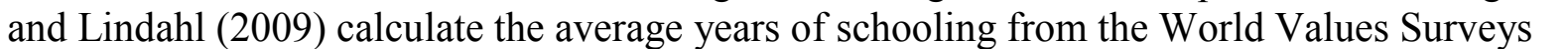

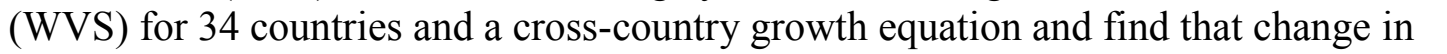

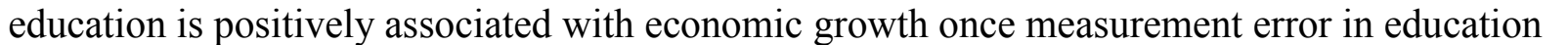

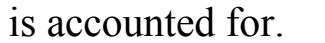

$\square$

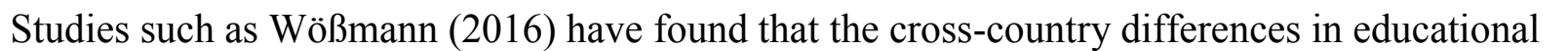

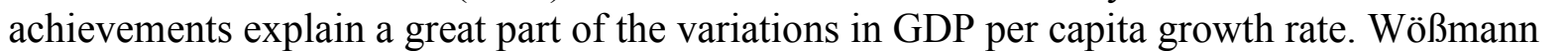
(2016) shows that a model with countries' average years of schooling and their initial level of

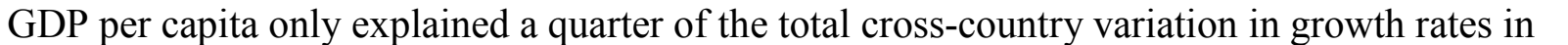

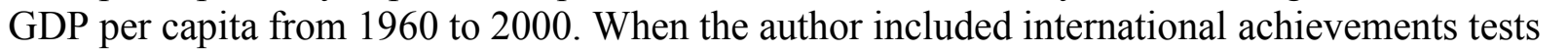

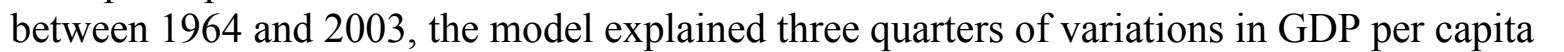

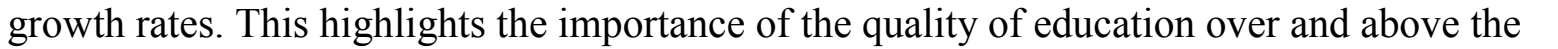

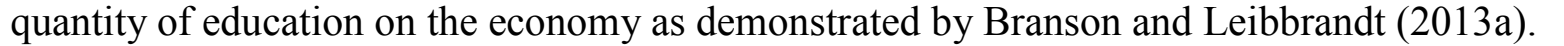

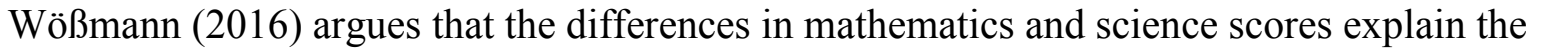

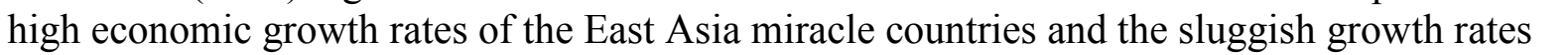

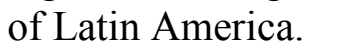

\section{B. Employment}

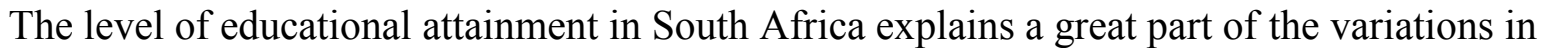

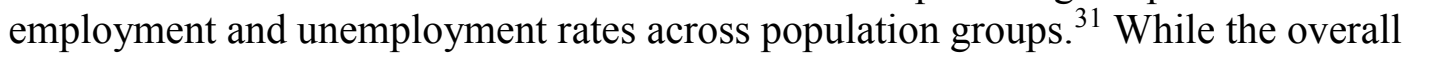

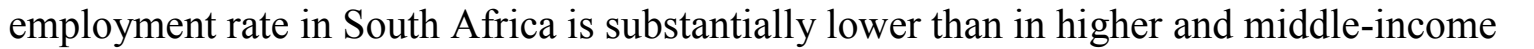

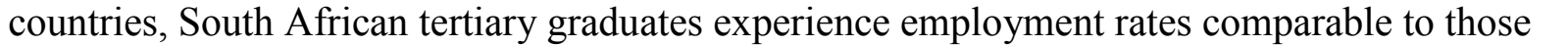
प

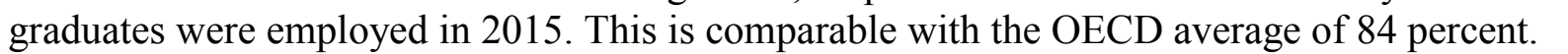

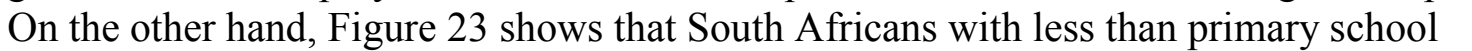

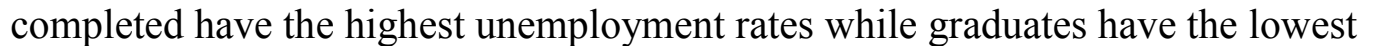

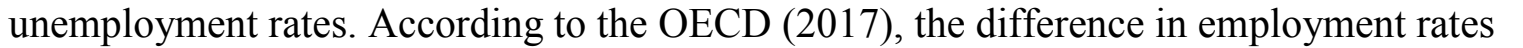

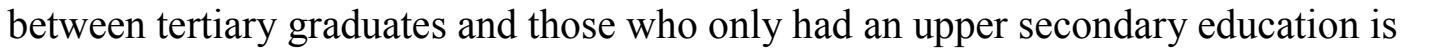

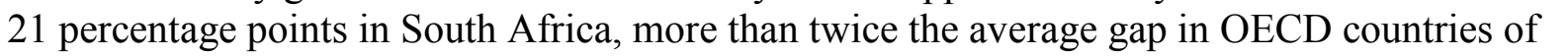

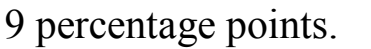

$\square$

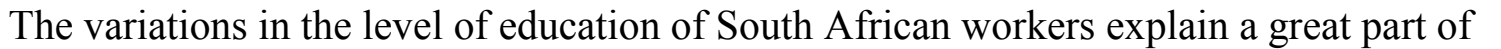

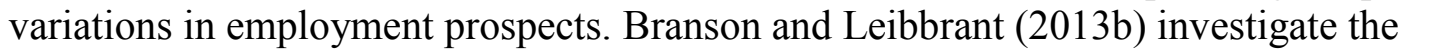

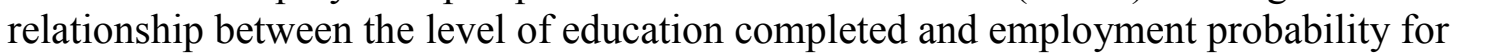

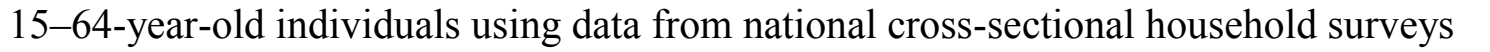

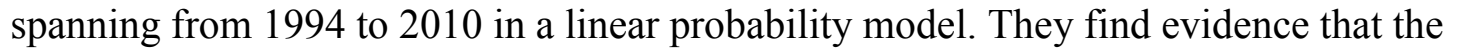

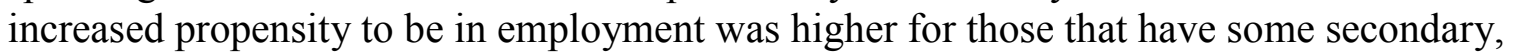

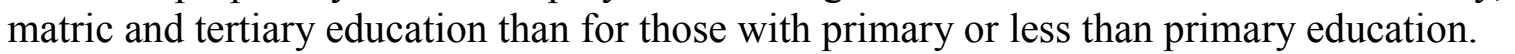

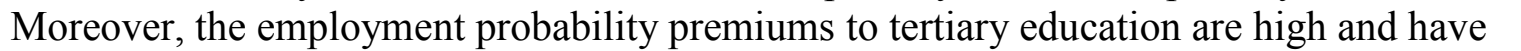

m

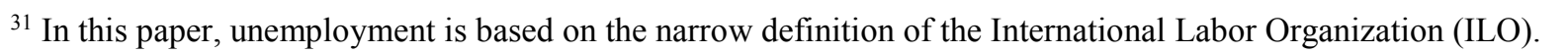

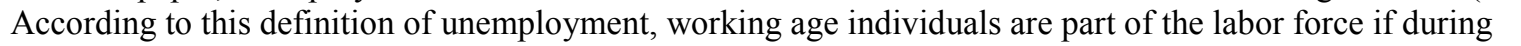

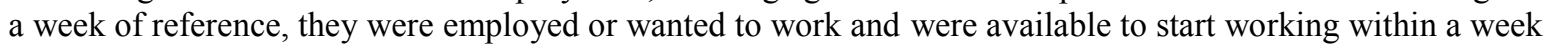

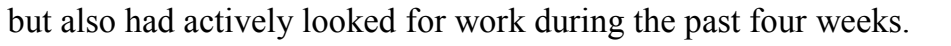




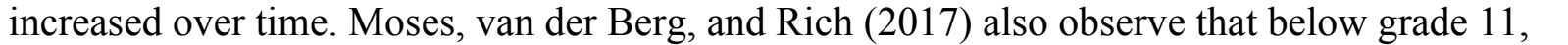
ए

$\square$

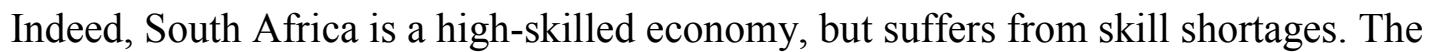

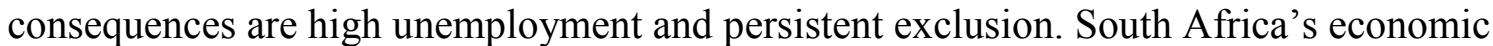

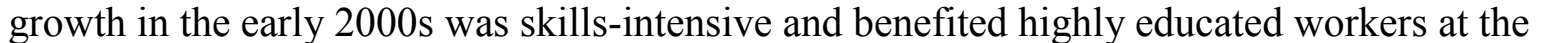

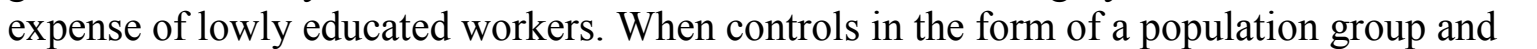

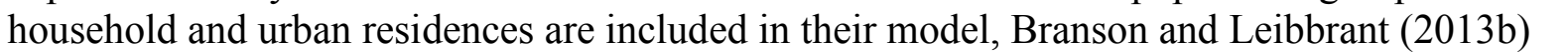

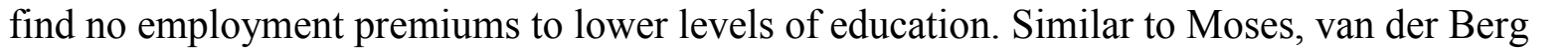

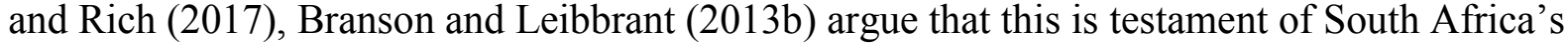

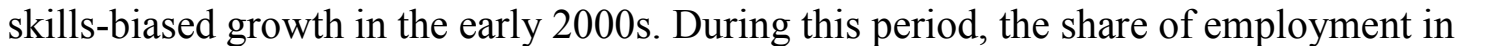

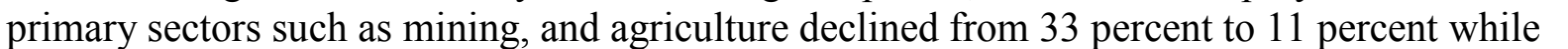
ए

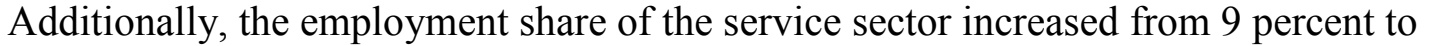

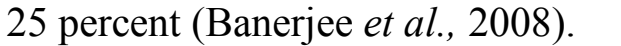

$\square$

Figure 22. Employment Rates of Population Aged Between 25 and 64 Years Who are Tertiary-Educated in 2015

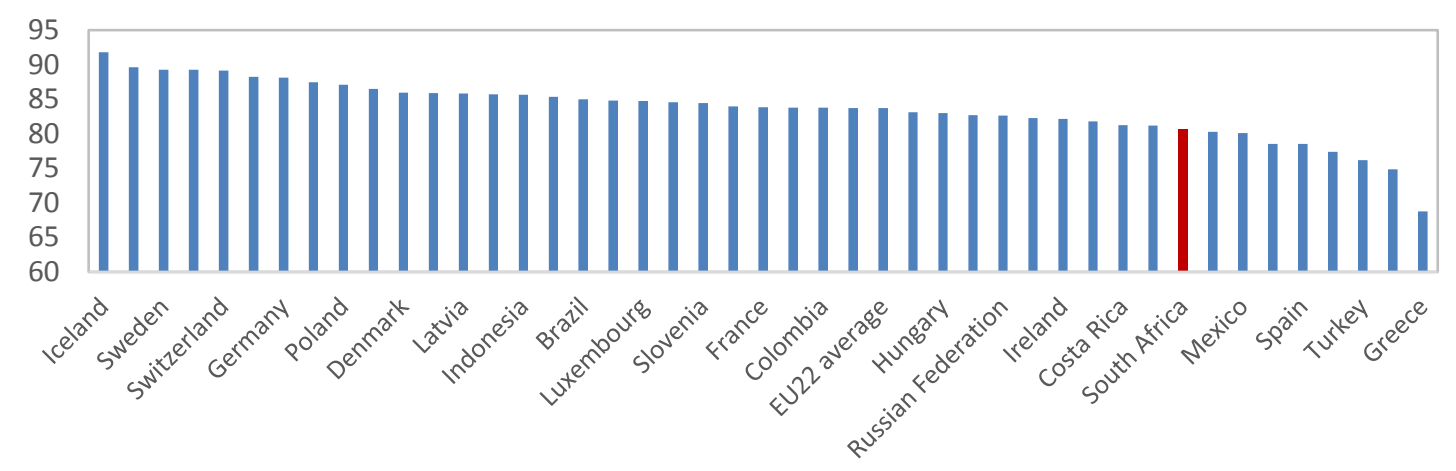

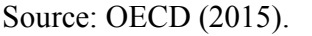

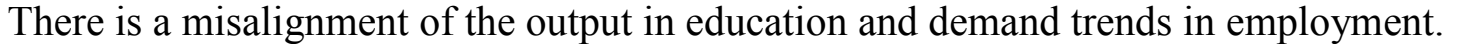

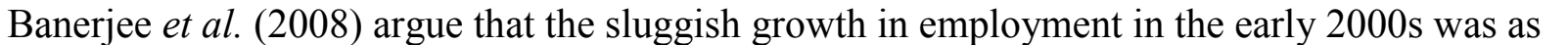

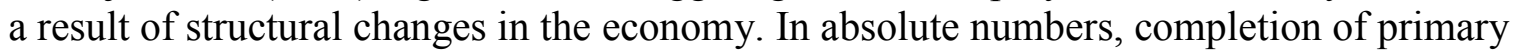

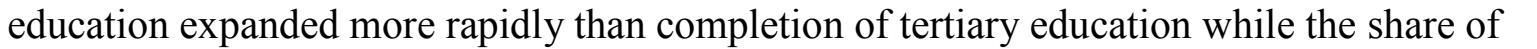

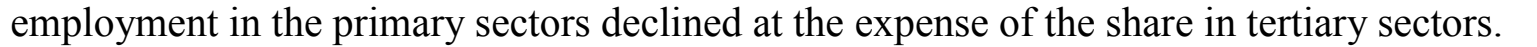

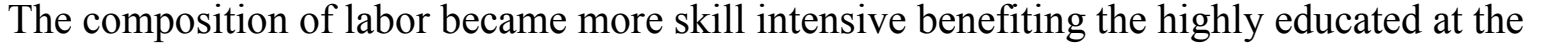

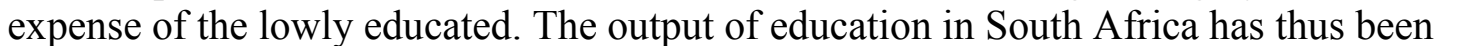

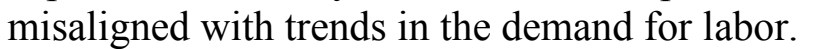

$\square$ 
Figure 23. Unemployment Rates by Level of Education for the Period 2008 to 2017

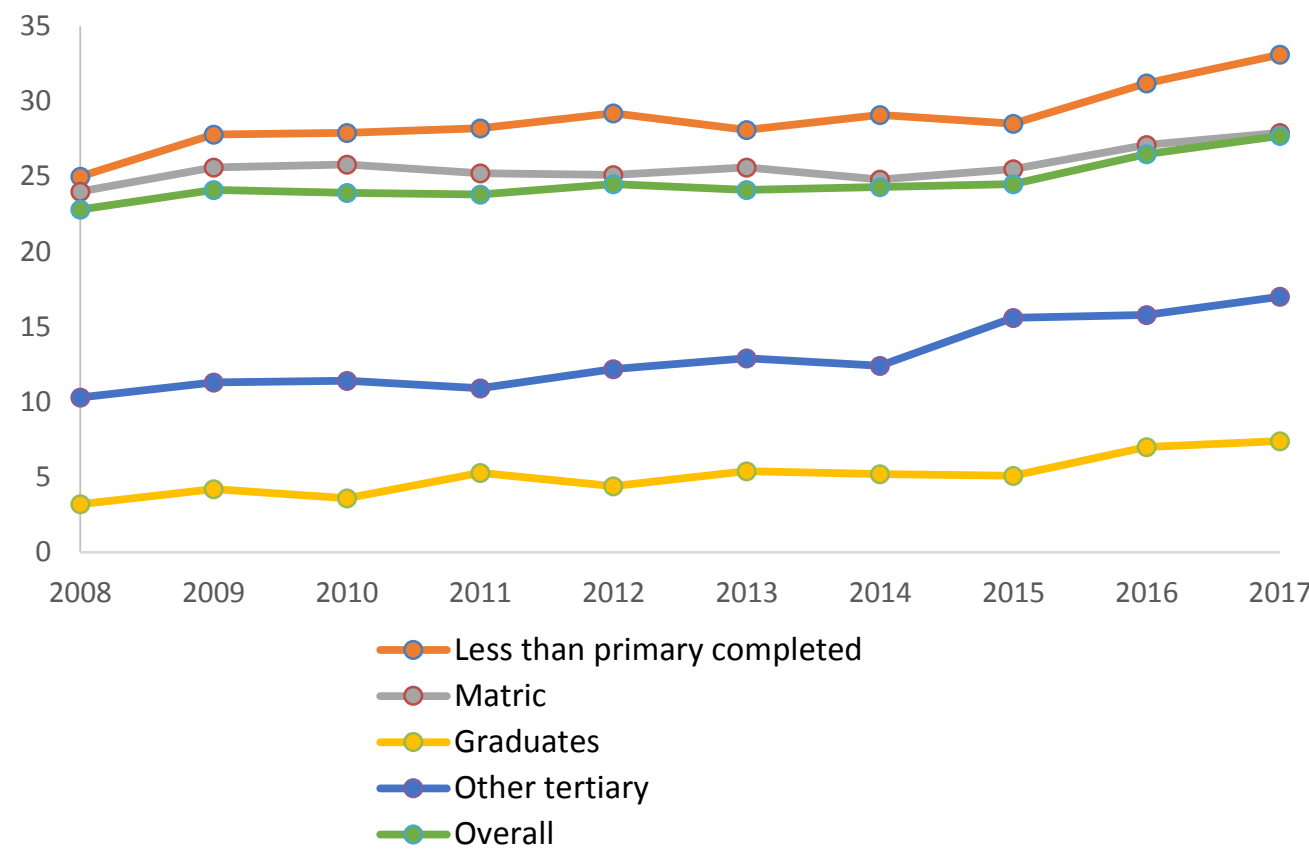

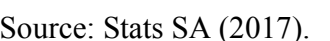

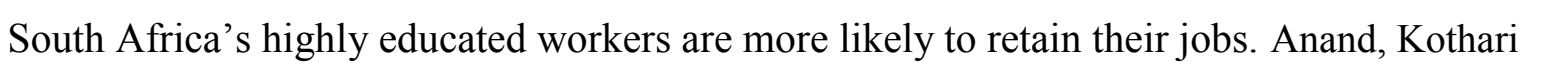

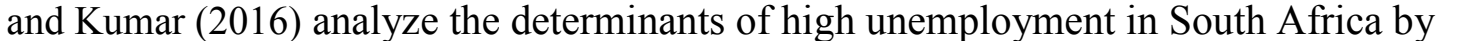

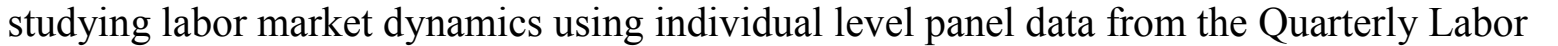

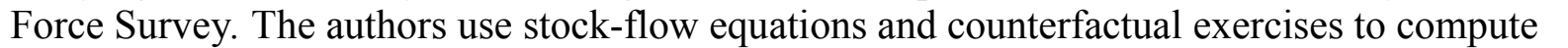

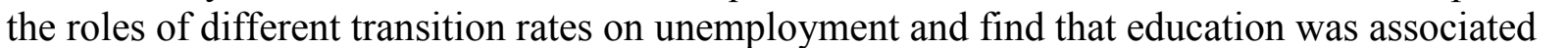

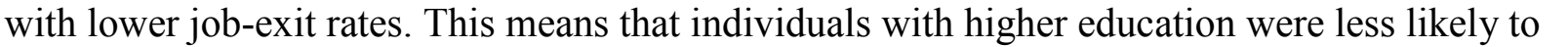

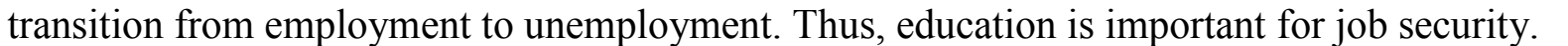

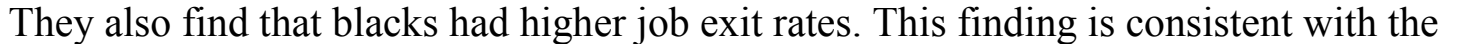

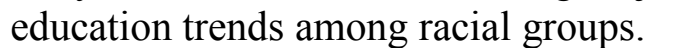

$\square$

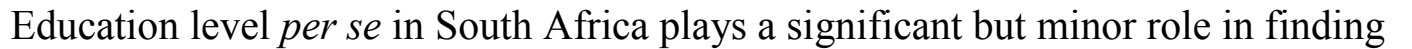

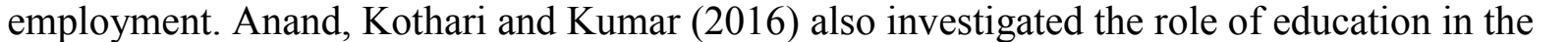

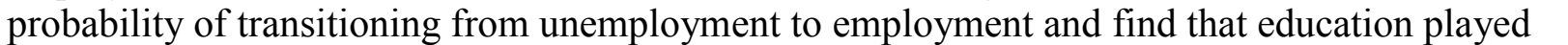

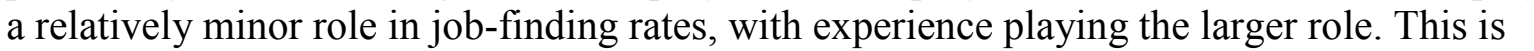
likely due to the fact that South Africa's primary and secondary education lags in quality and

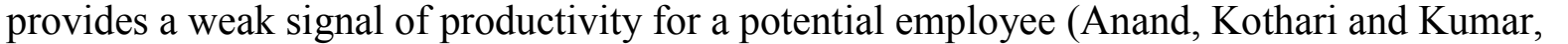
$\square \square \square \|$

$\square$

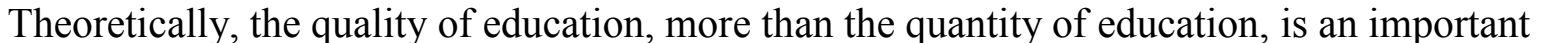

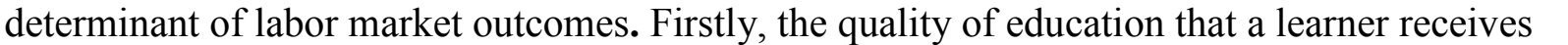

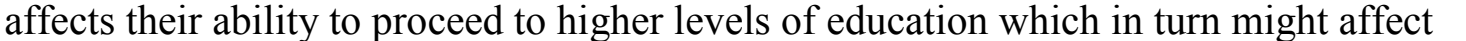

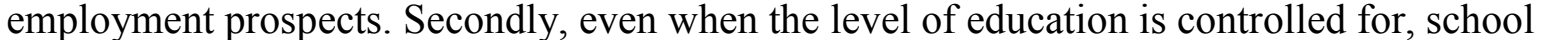

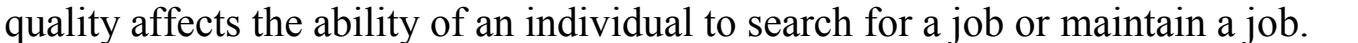

$\square$

CInternational Monetary Fund. Not for Redistribution 
एव

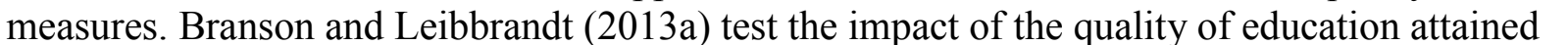

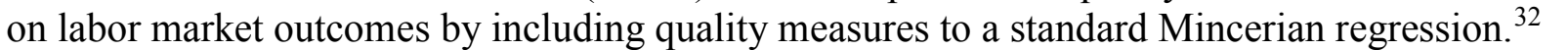

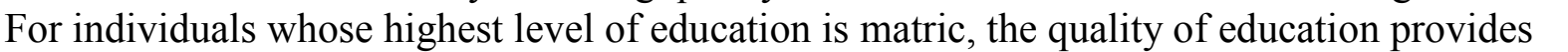

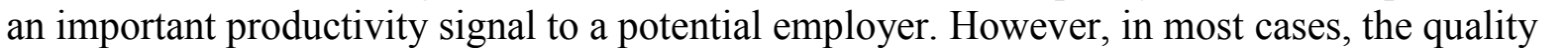

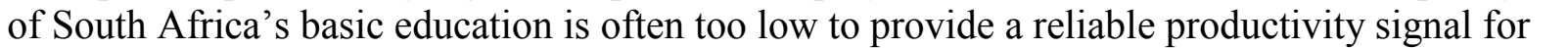

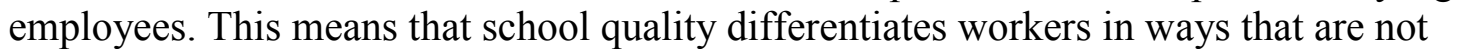

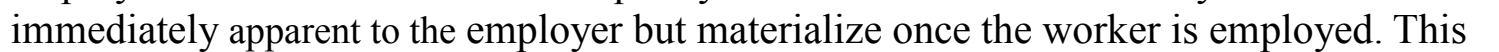

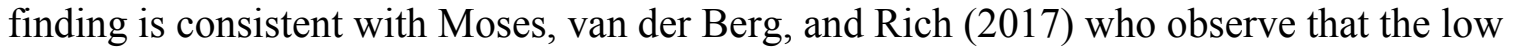

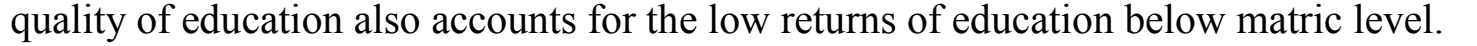
$\square$

\section{Earnings, Inequality, and Poverty}

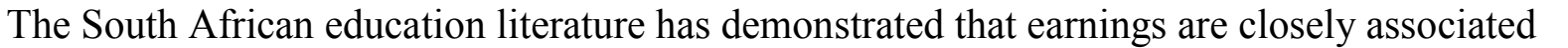

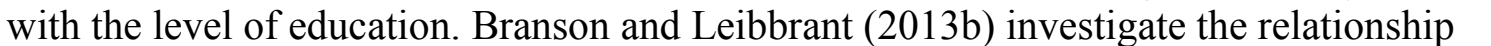

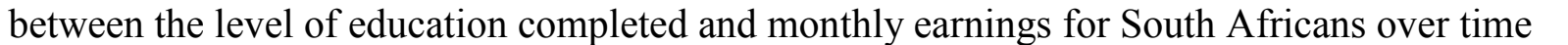

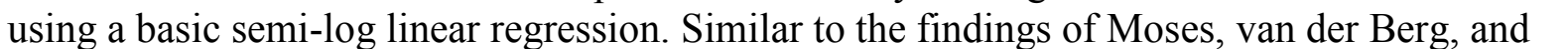

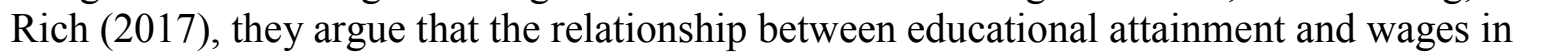

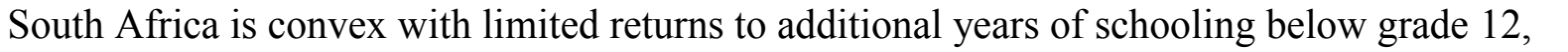

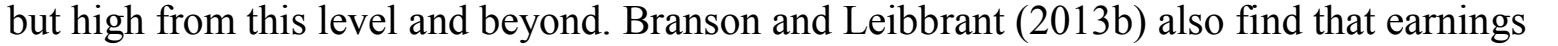

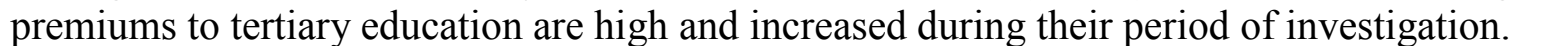

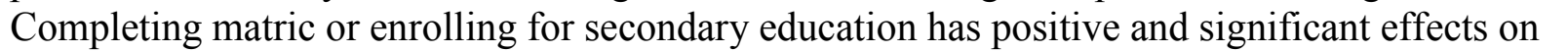

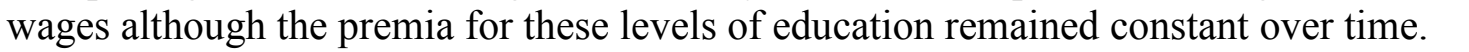
$\square$

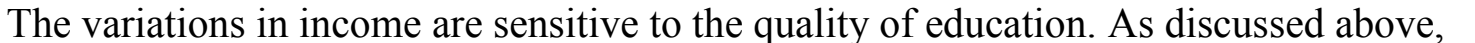

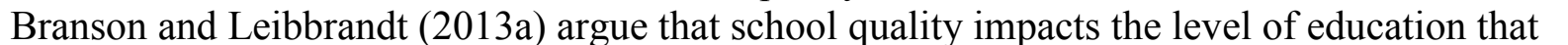

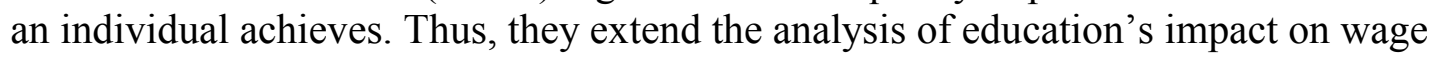

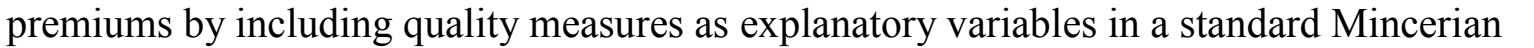

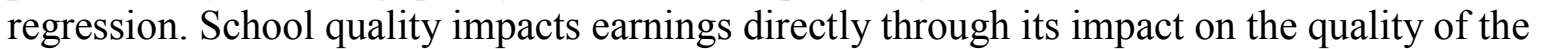

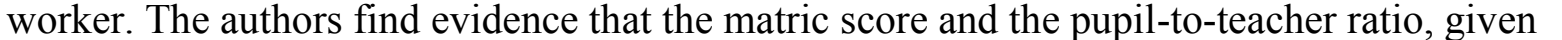
$\square$ respondent's closest school, has an impact on earnings. A 10 percent increase in the matric

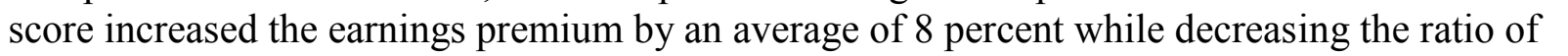

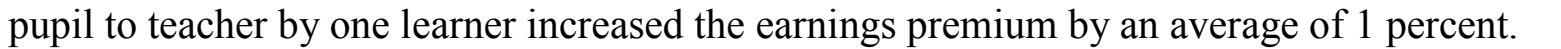
$\square$

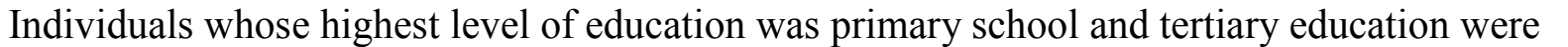

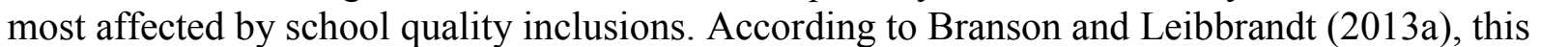

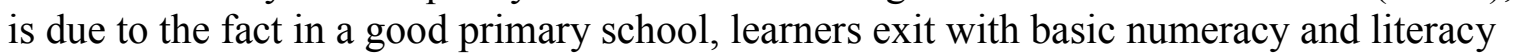

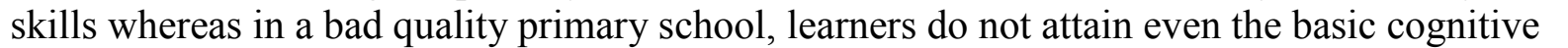

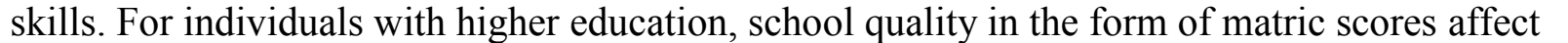

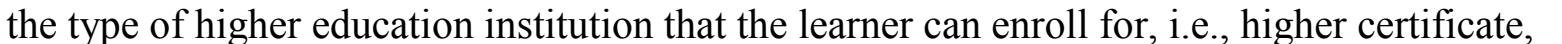
diploma or degree, which in turn affects the individuals' wages upon finding employ $\square \square \square \square \square \square$ der Berg (2005) also finds evidence that a major part of South Africa's residual earnings

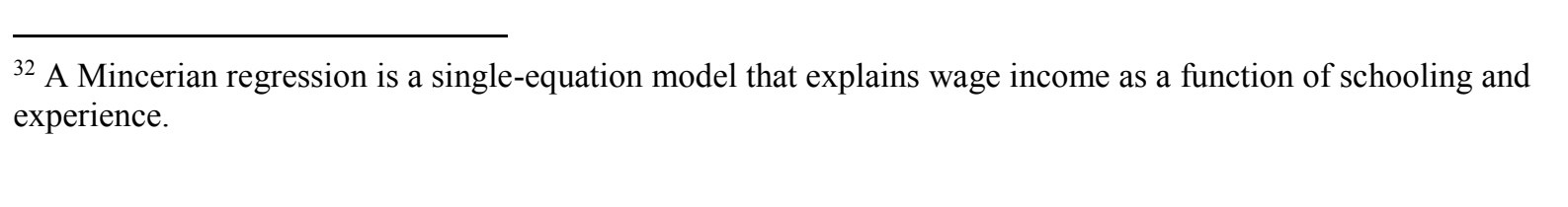




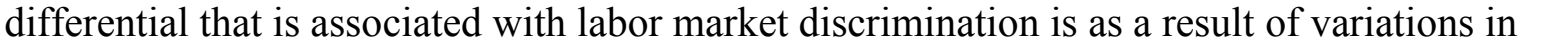

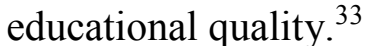

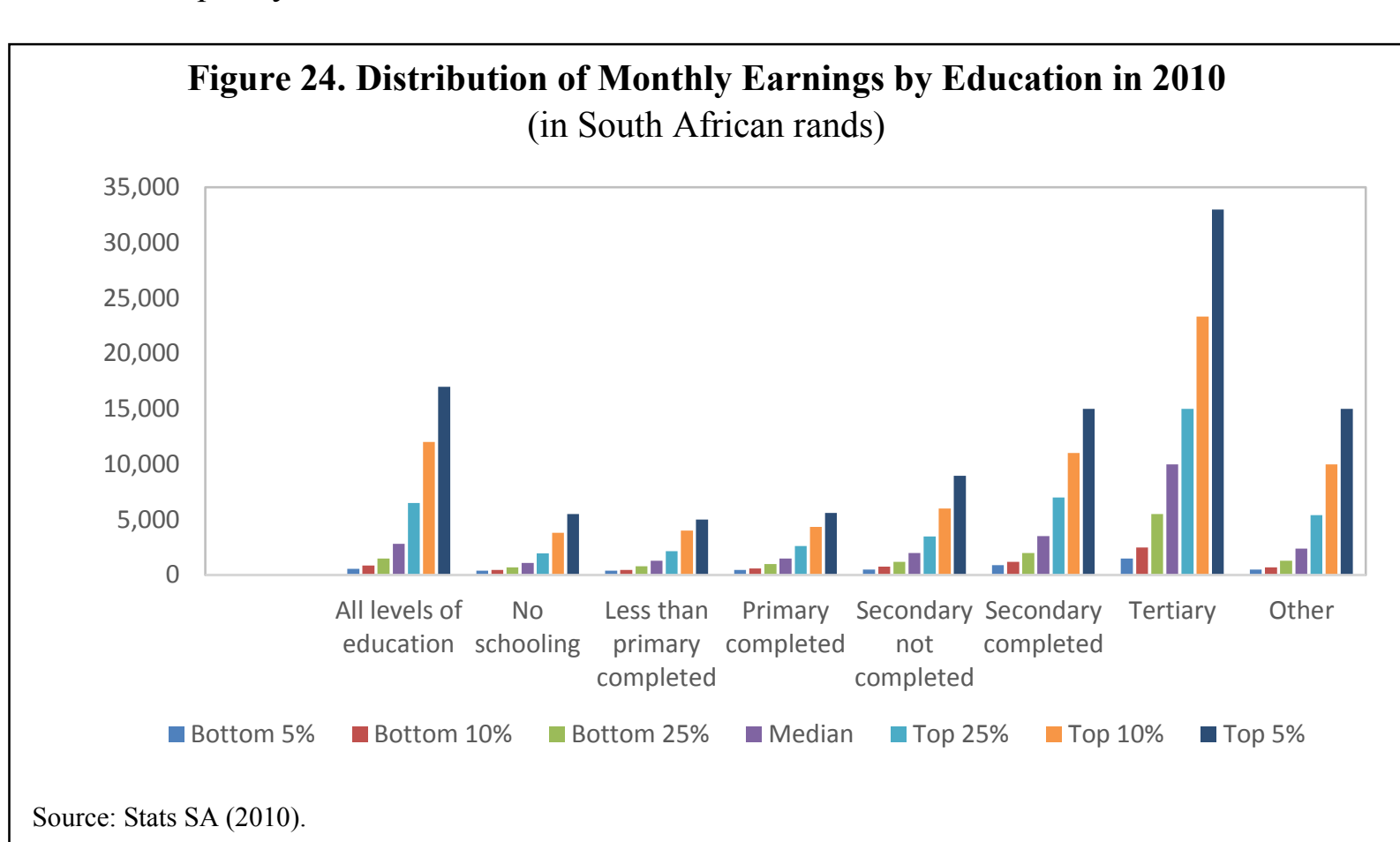

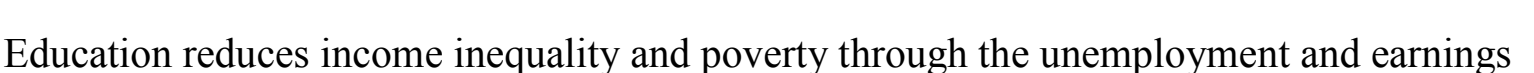

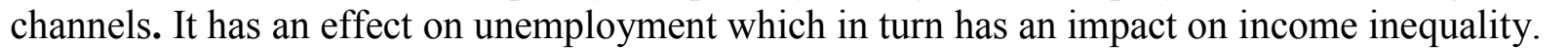

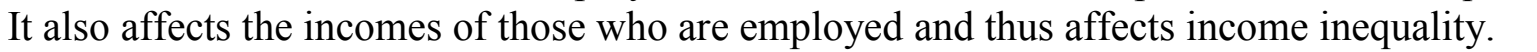

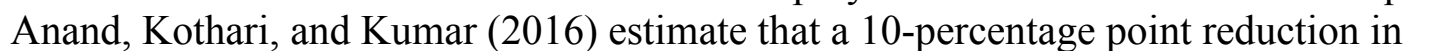

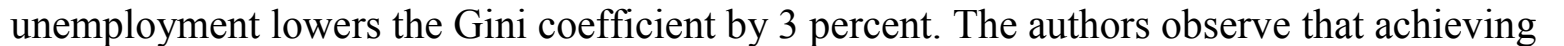

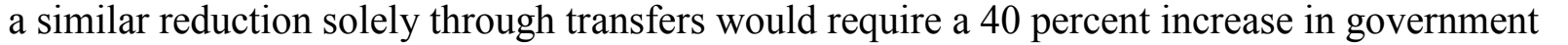

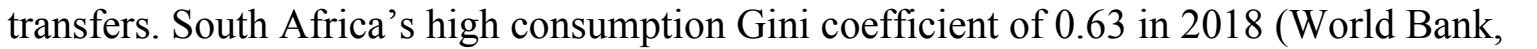

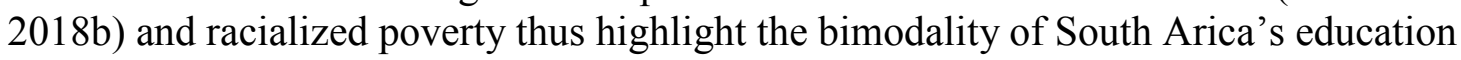

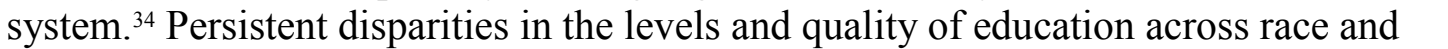

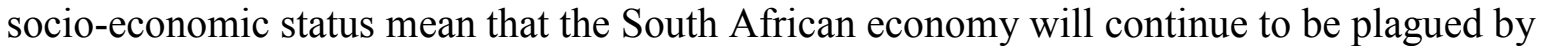

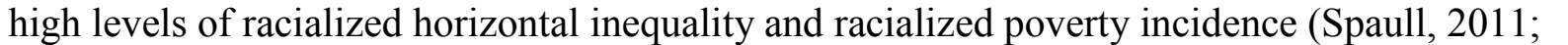

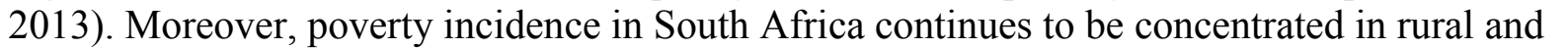

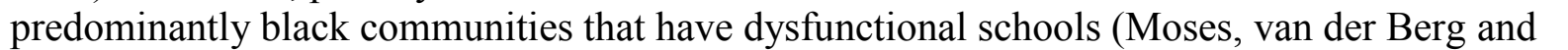

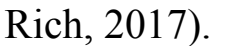
$\square$

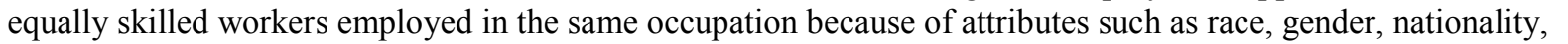

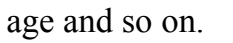

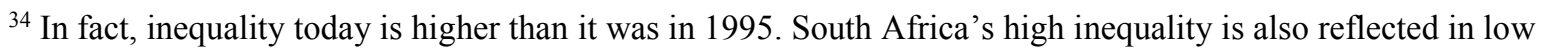

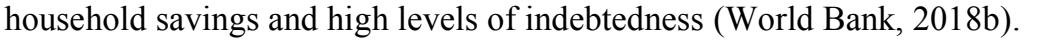


Figure 25. Poverty Incidence of Households by Education Level of Household Head

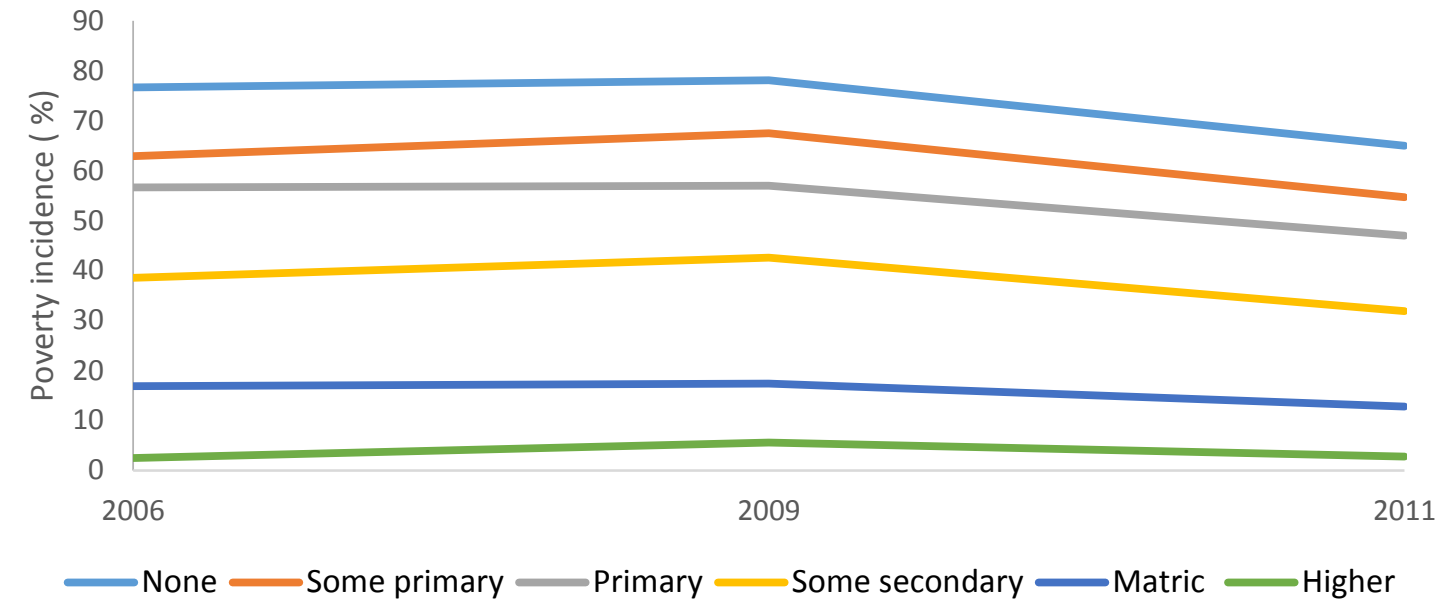

$\square$

\section{Policy Considerations}

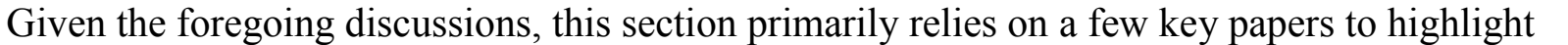

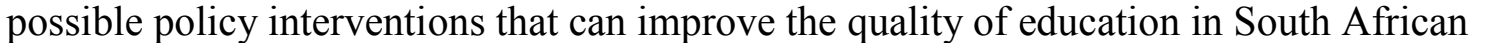

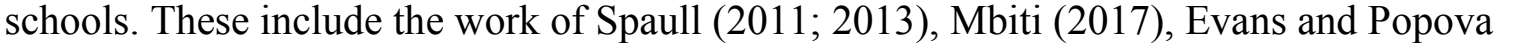

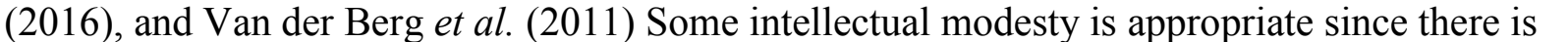

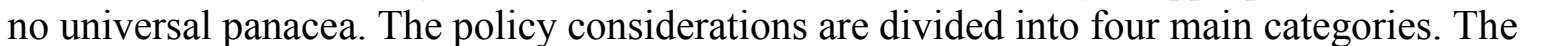

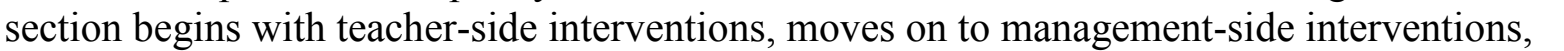

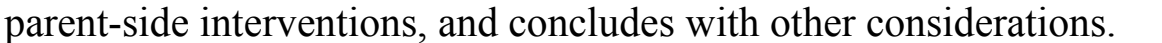
$\square$

\section{A. Teacher-Side Interventions}

\section{Capacity building}

South Africa's policymakers should consider implementing more intensive, continuous, and

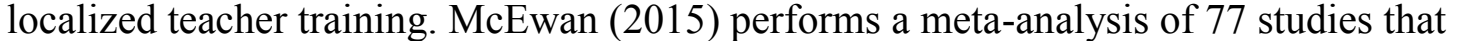

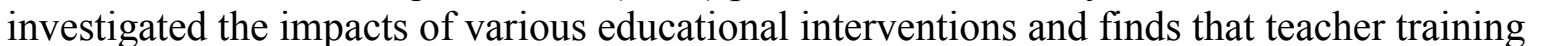

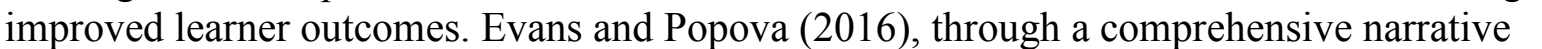

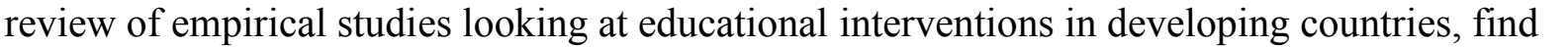

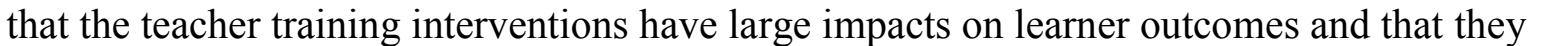

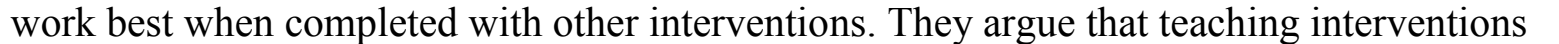

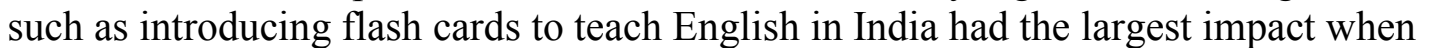

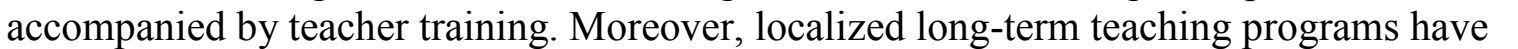
प

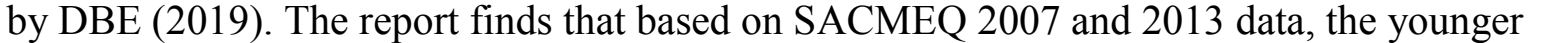

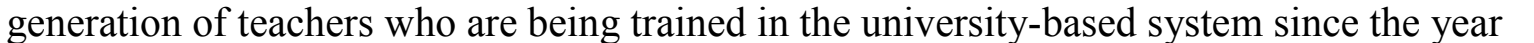

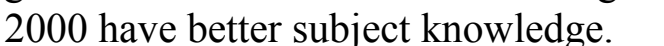

$\square$

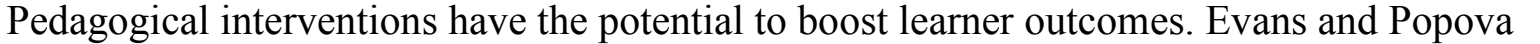
ए। 


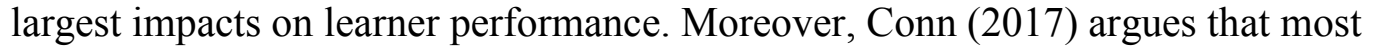

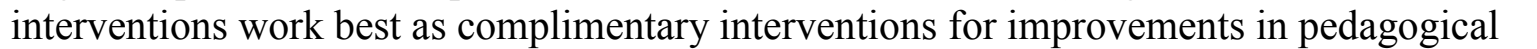

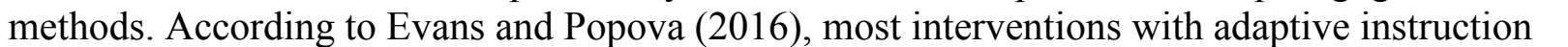

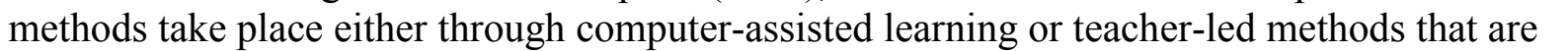

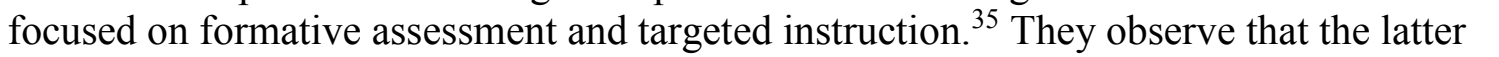

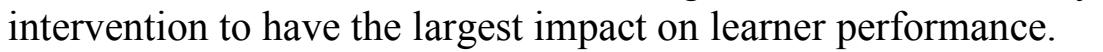

$\square$

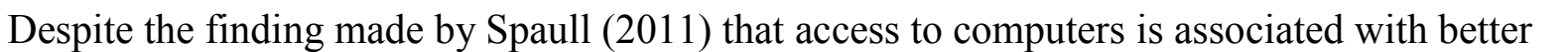

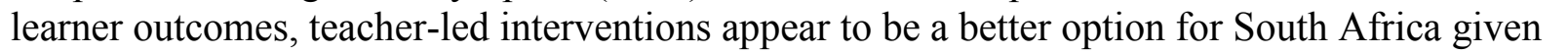

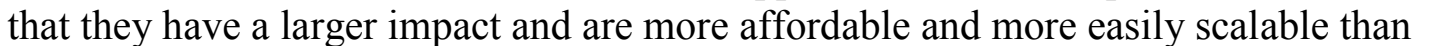

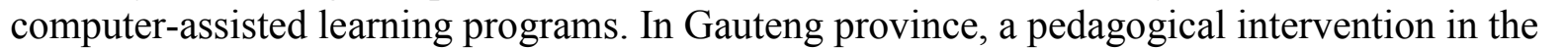

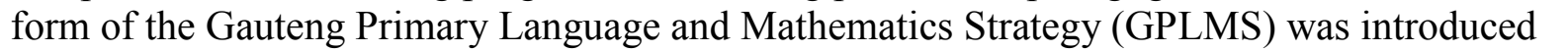

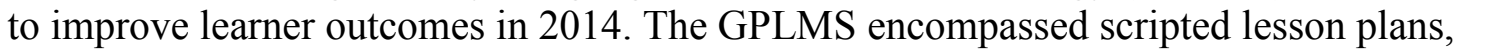

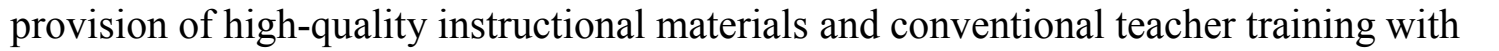

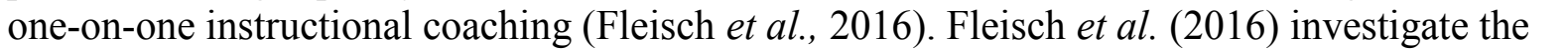

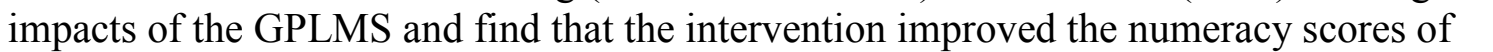
पाणाणाएण

\section{Performance pay}

$\square$

$\llbracket \llbracket$ asing accountability by transforming South Africa's teacher pay system from a flat pay

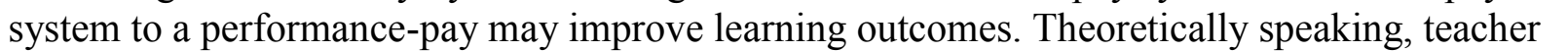

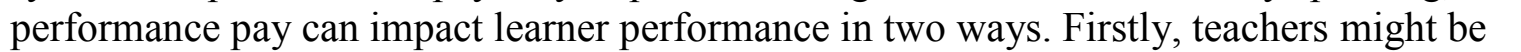

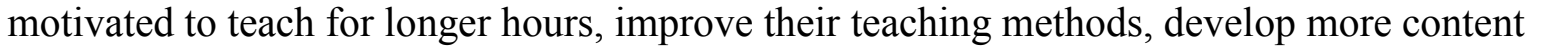

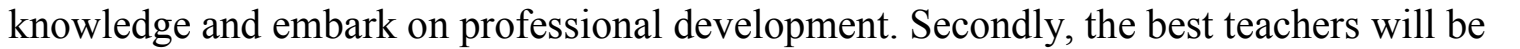

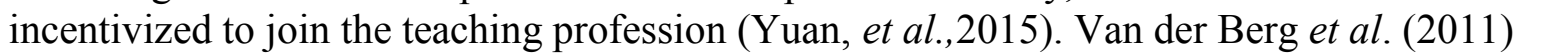

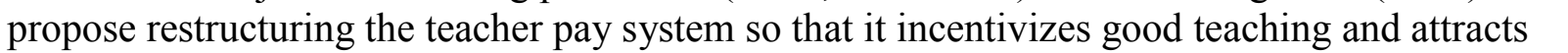

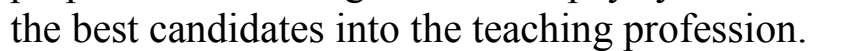

$\square$

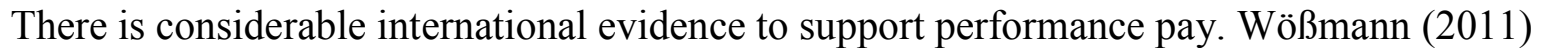

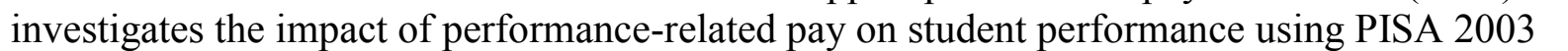

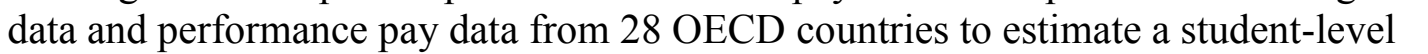

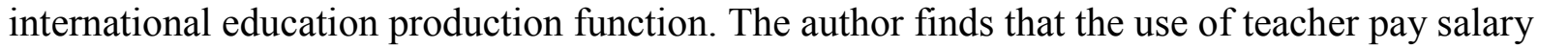

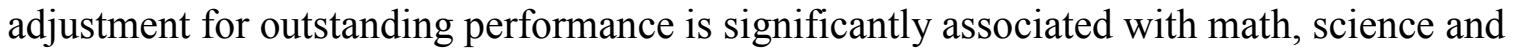

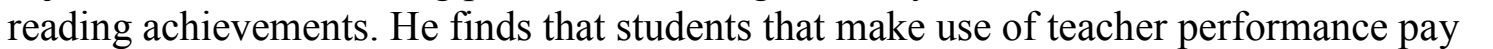

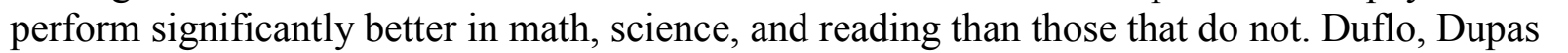

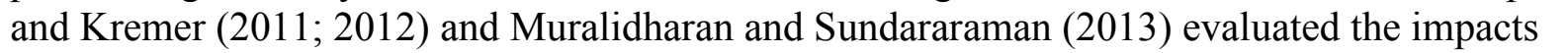

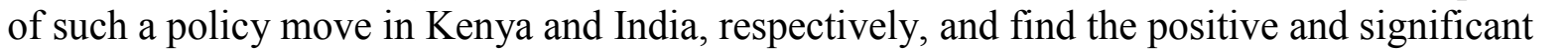

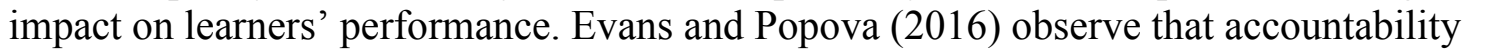

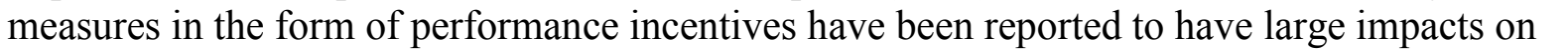
एणमाणमाणमाण

$\square$

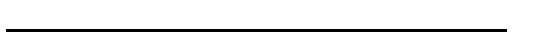

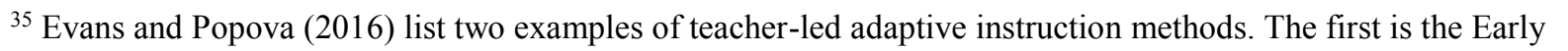

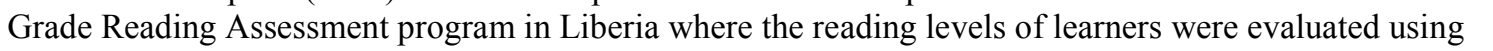

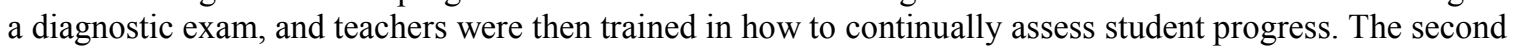

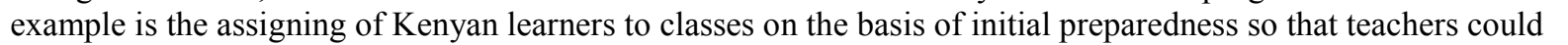

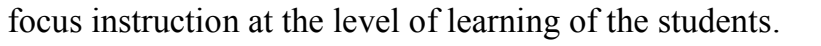




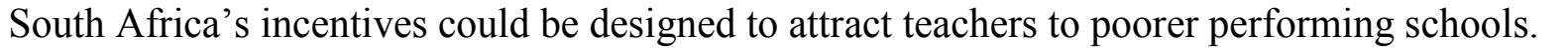

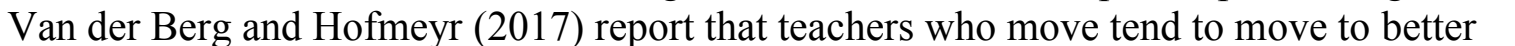

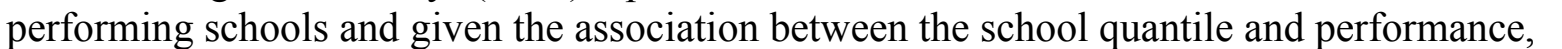
ए

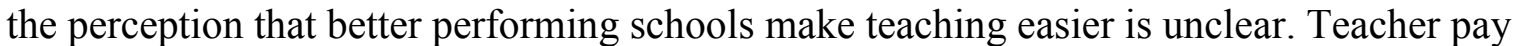

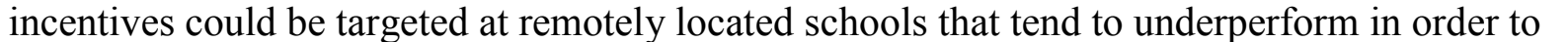

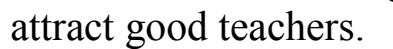

$\square$

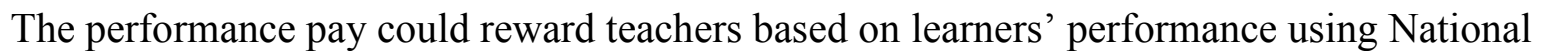

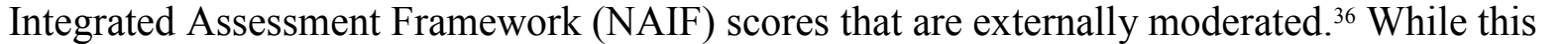

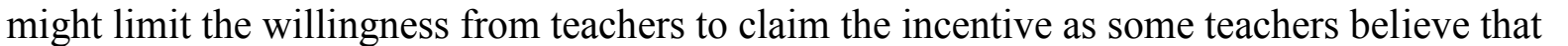

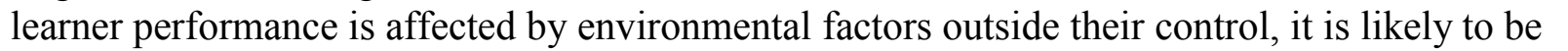

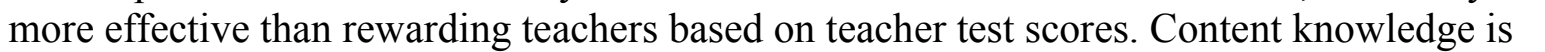

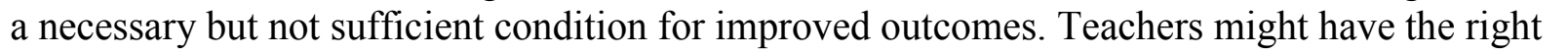

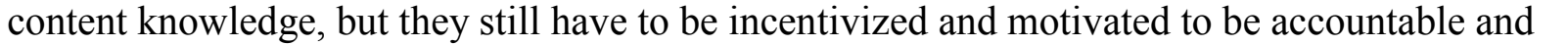

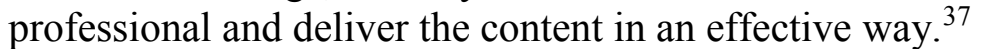

$\square$

\section{B. Management-Side Interventions}

\section{School management}

$\square$

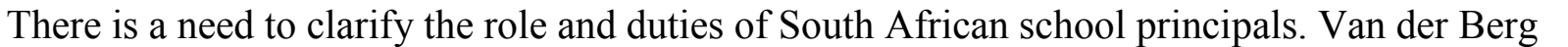

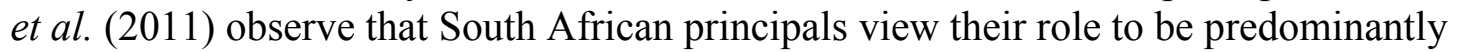
managerial and administrative. Principals' roles should be extended to include instruct $\square \square \square$

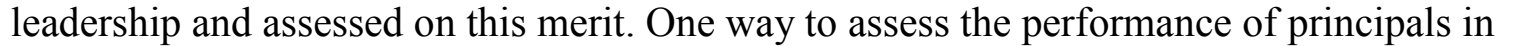

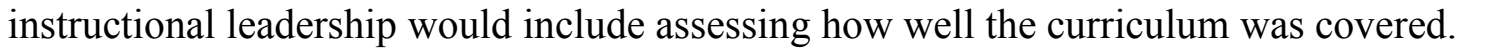

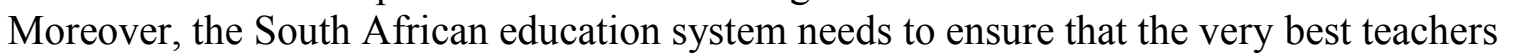

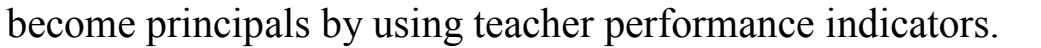

$\square$

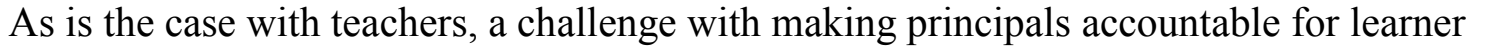

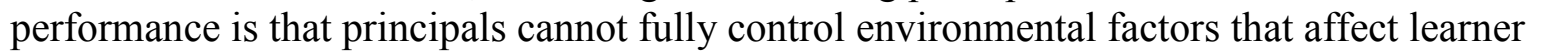

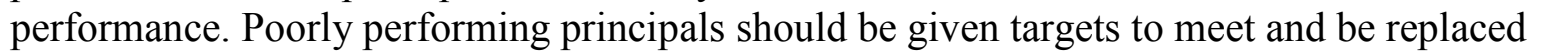

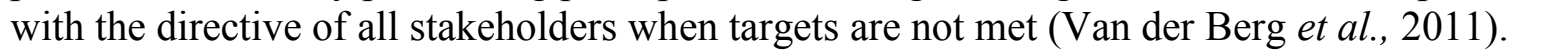

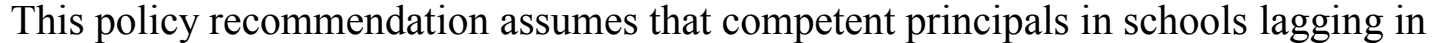

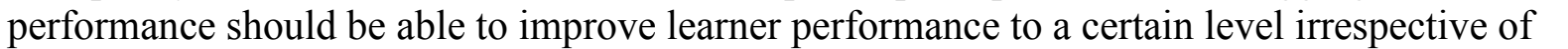

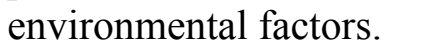

$\square$

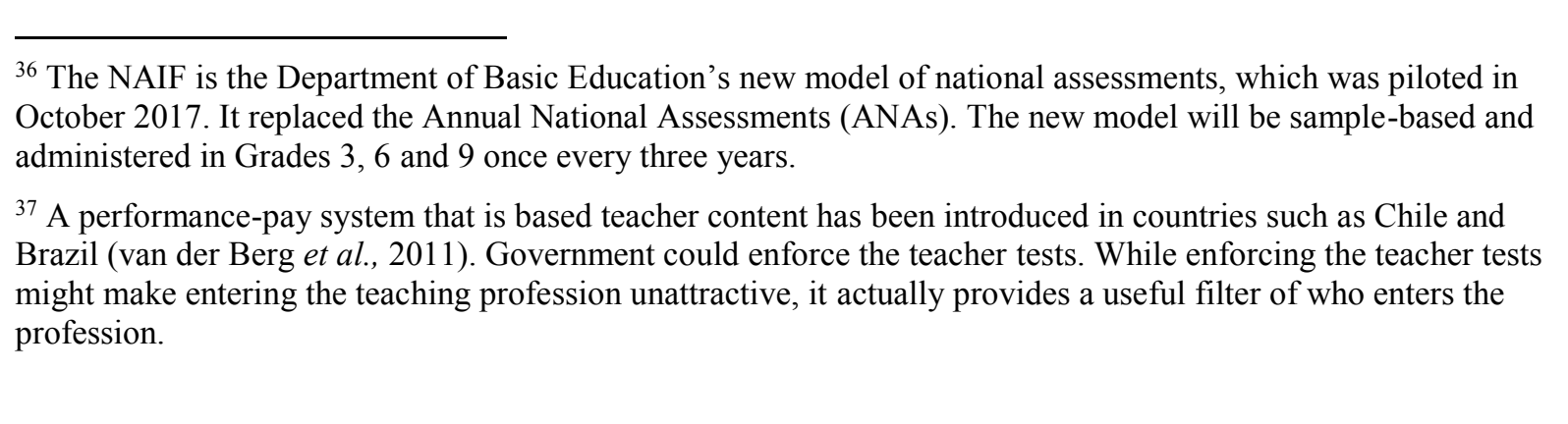

(CInternational Monetary Fund. Not for Redistribution 


\section{Parent-Side Interventions}

\section{Greater community involvement}

$\square$

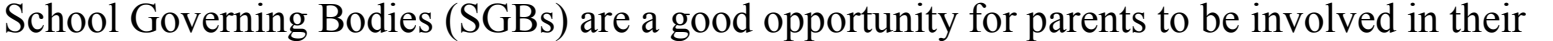

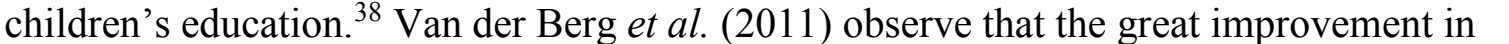

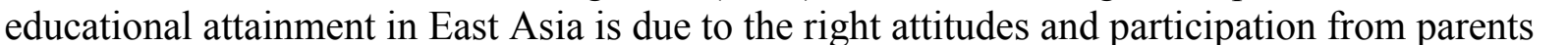

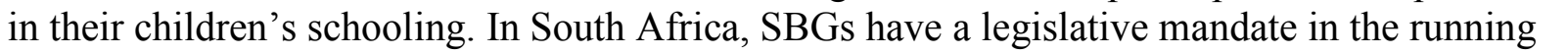

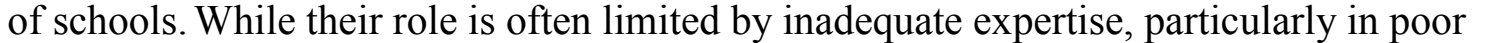

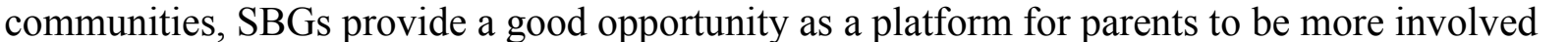

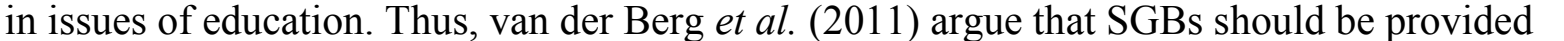

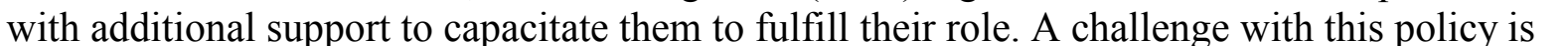

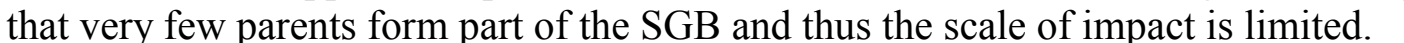
$\square$

A possible measure to make all parents take interest in their children's education is to require periodic meetings with teachers to review children's performance. Such meetings could tak $\square$

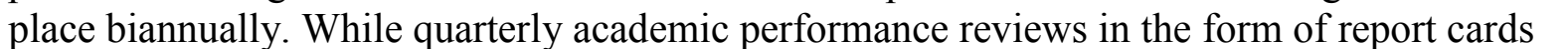

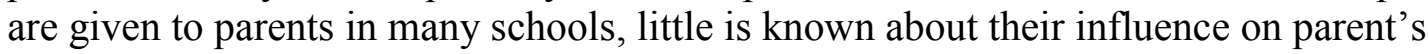

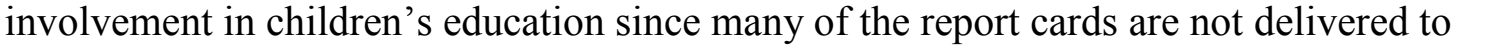

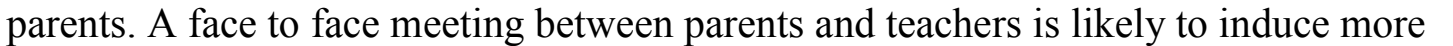
intervention from parents since it also allows teachers to report to parents about children's

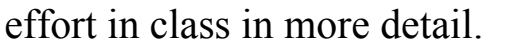
$\square$

\section{Other Interventions}

\section{External moderation of the National Integrated Assessment Framework}

$\square$

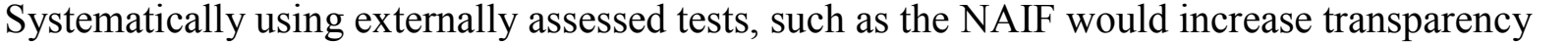

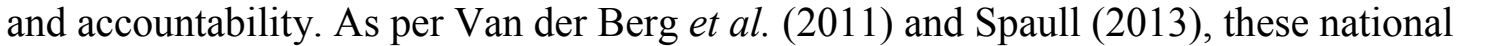

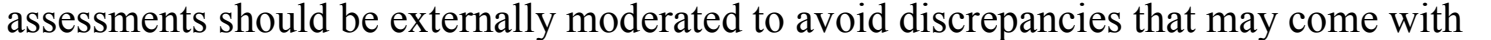

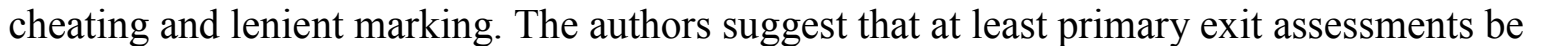

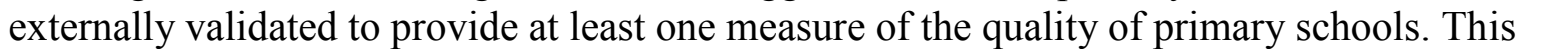

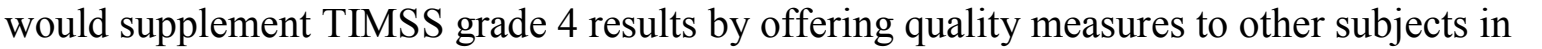

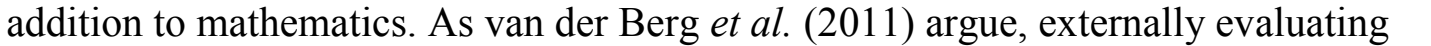

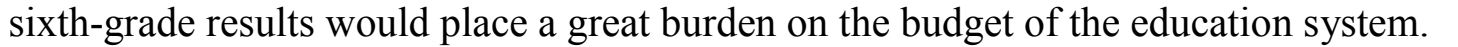

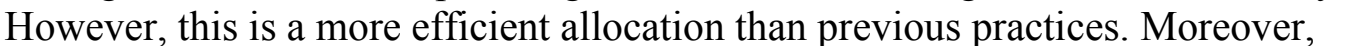

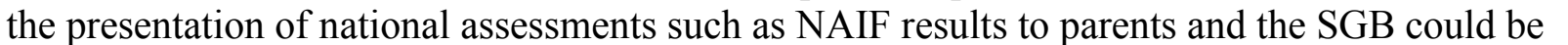
improved so that parents have better information about their children's per $\square \square \square \square \square \square \square \square \square \square \square\|\|$ $\square \square$ et al., 2011). The results report could contain information about the child's performance

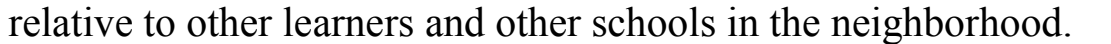

$\square$

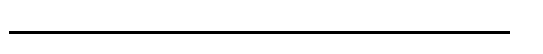

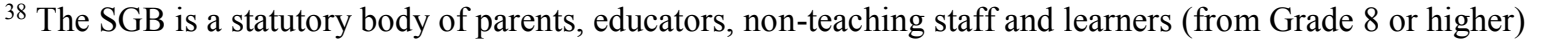

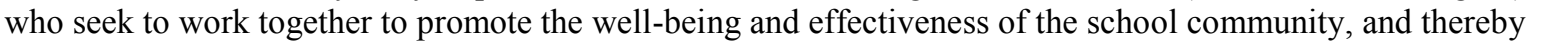

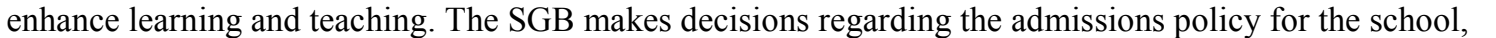
ए

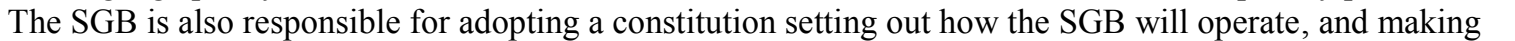

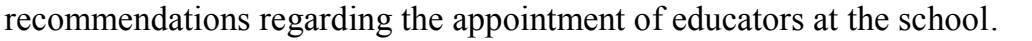




\section{Language}

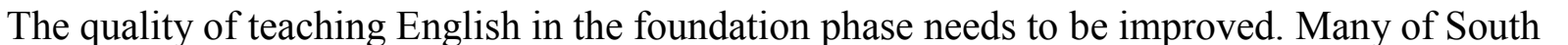
Africa's learners do not use English as their language of communication in their homes.

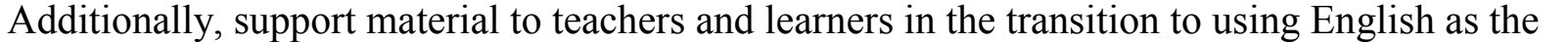

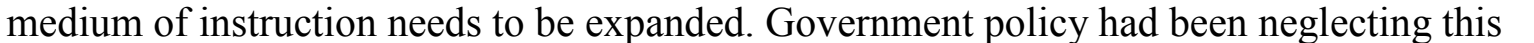

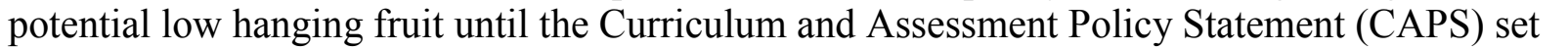

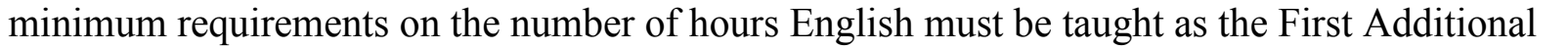

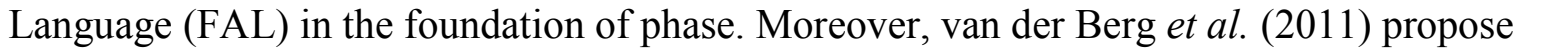

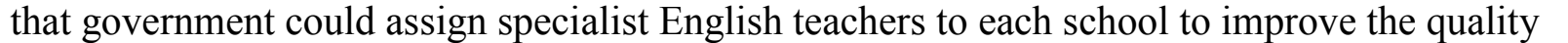

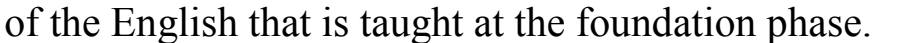

$\square$

\section{Early Childhood Development}

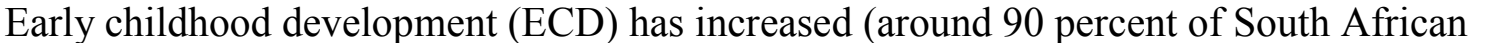

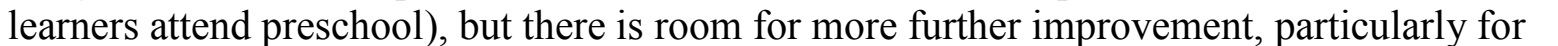

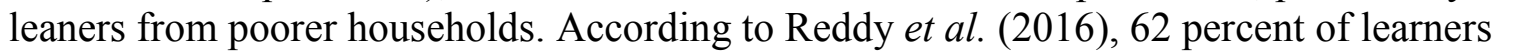

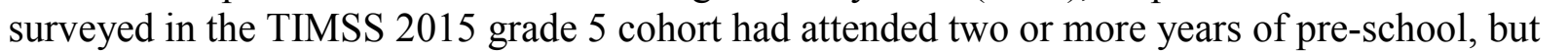
ए

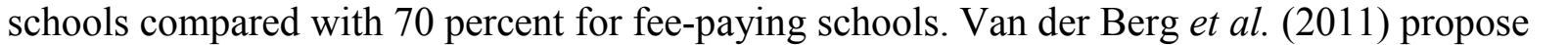

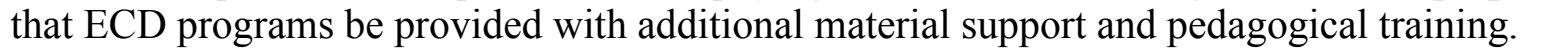

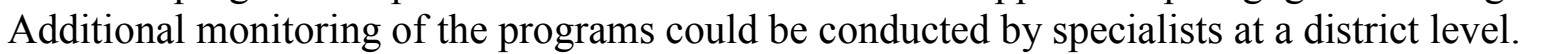

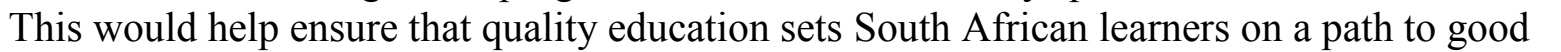

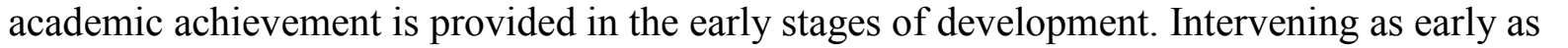

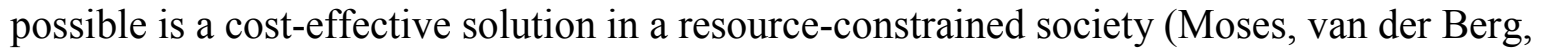

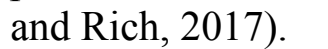

\section{Low-hanging fruits}

$\square$

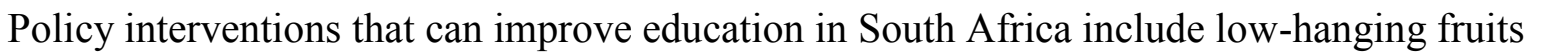

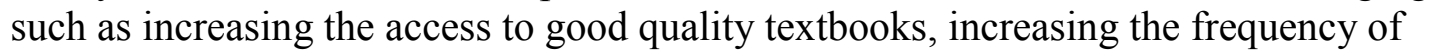

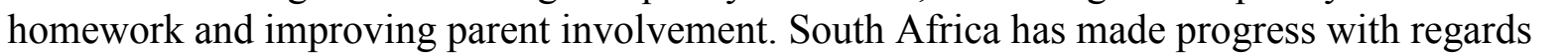

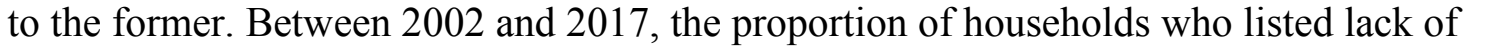

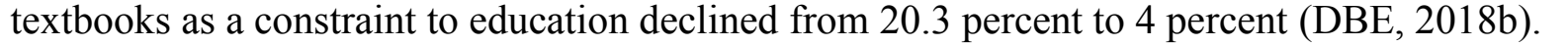

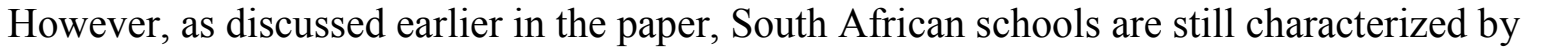
ए

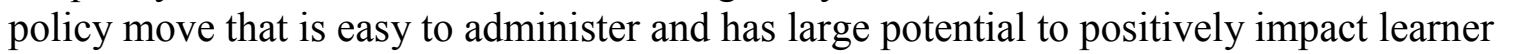

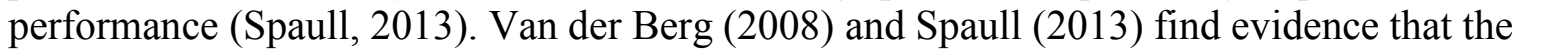
ए

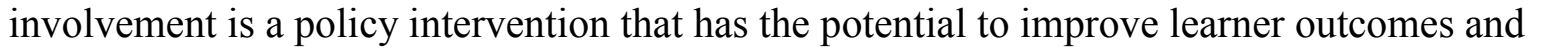

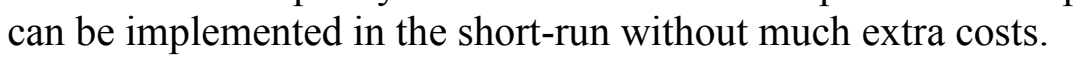
$\square$

\section{E. Summing Up}

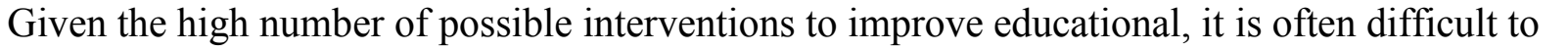

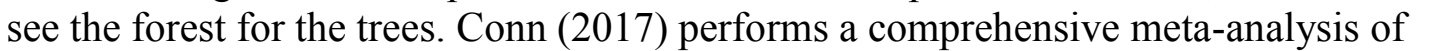

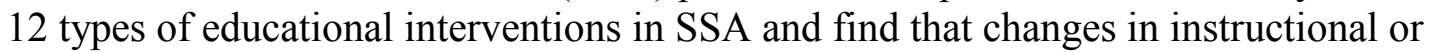




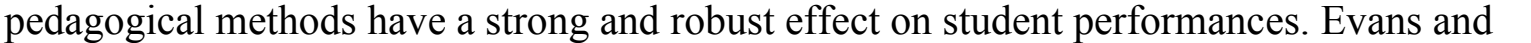

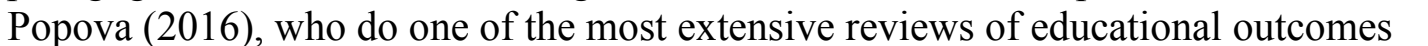

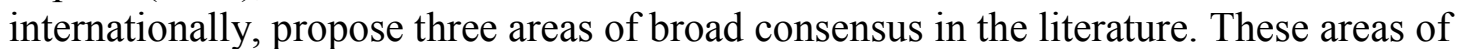

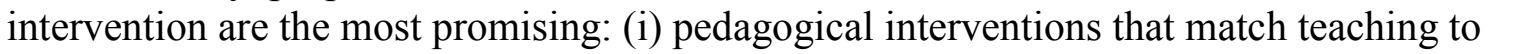

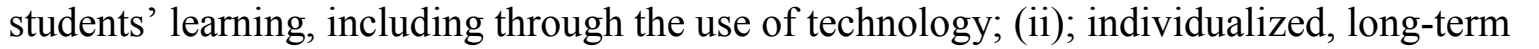

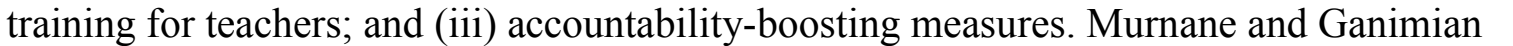
ए

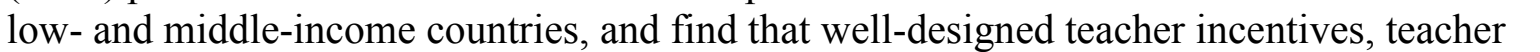

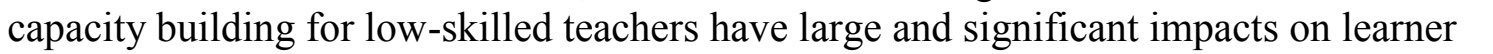

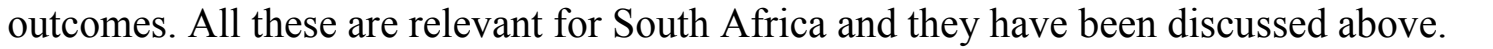

\section{CONCLUding REMARKS}

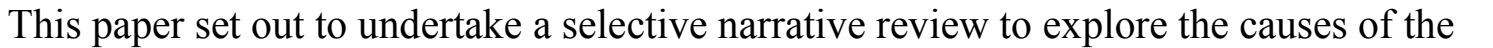

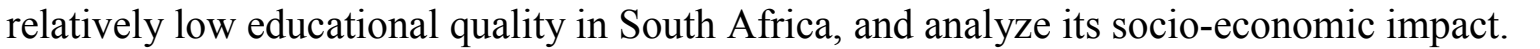

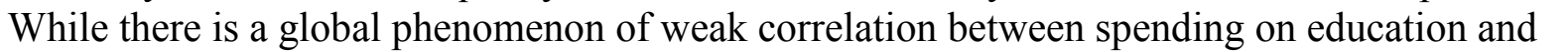

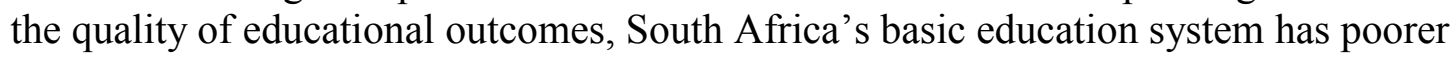

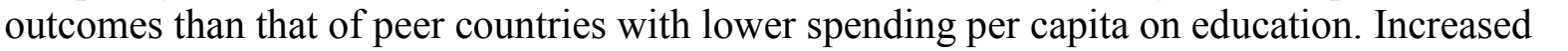

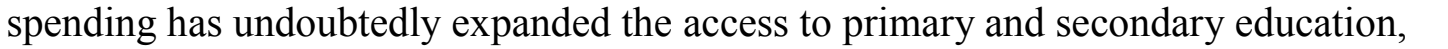

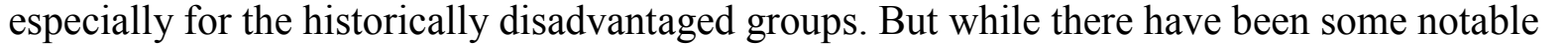

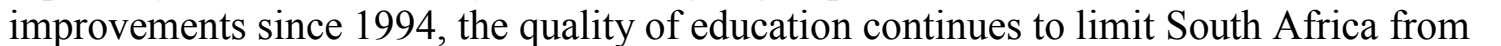

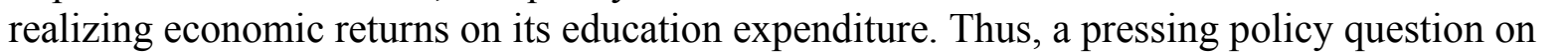
South Africa's education system is how resources can be used more efficiently to achieve

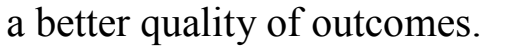

$\square$

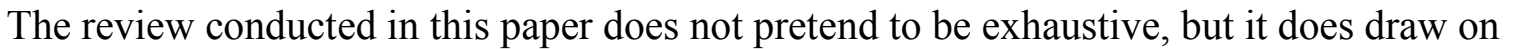

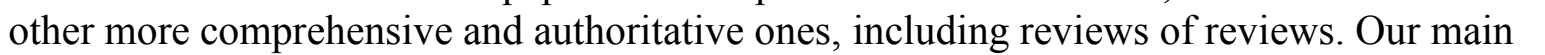

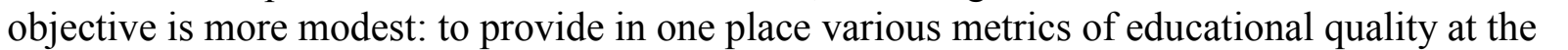

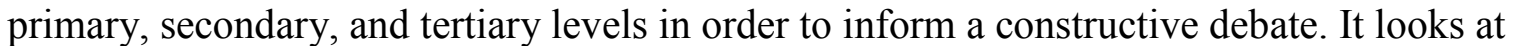

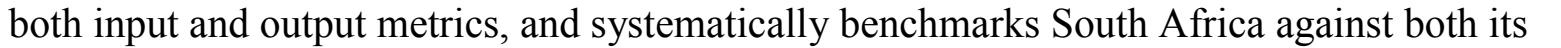

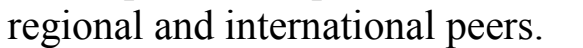

$\square$

It is safe to say that there is no panacea for improving outcomes in South Africa's education

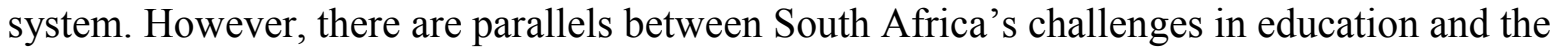

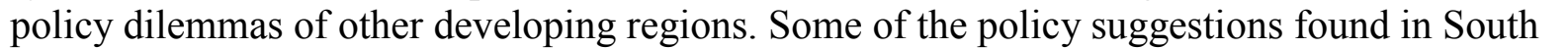

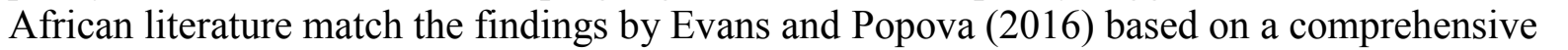

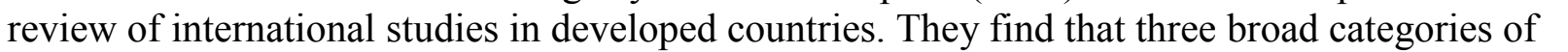

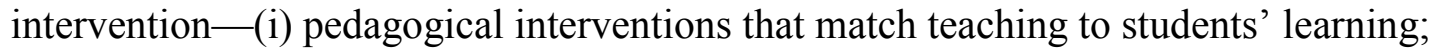

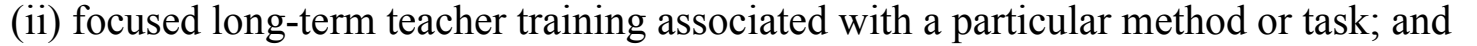

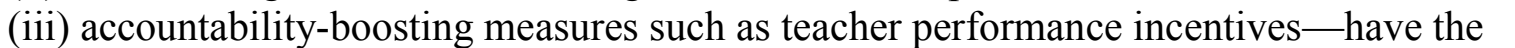

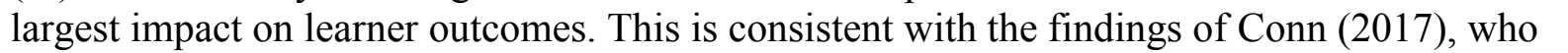

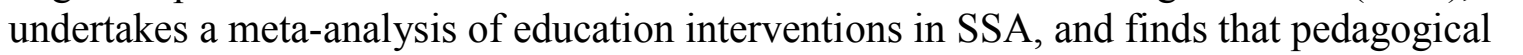

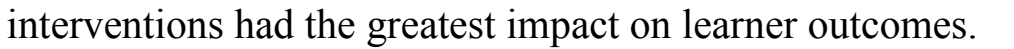

$\square$

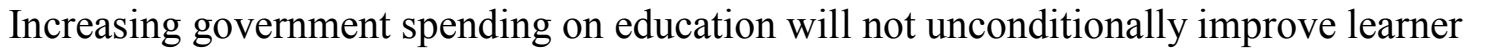

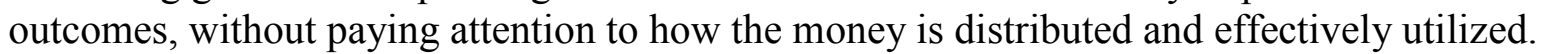

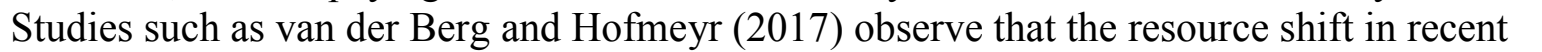




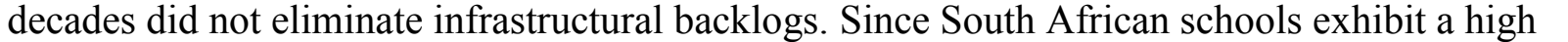

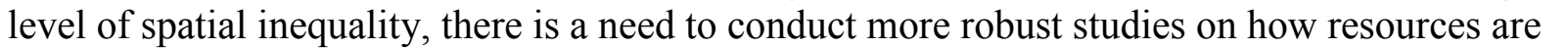
$\square$ stributed within South Africa's education system. These studies should aim to enable $\square \square \square \square$

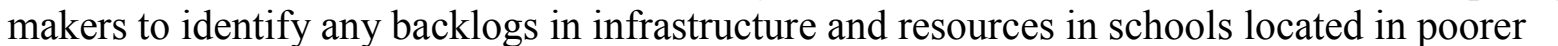

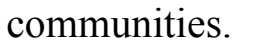

$\square$

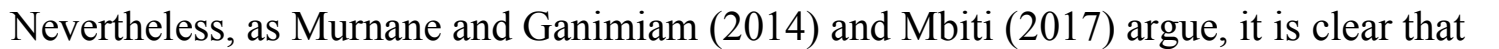

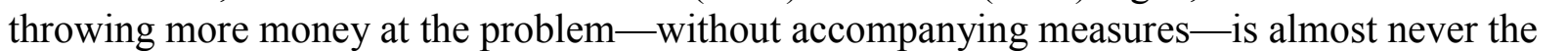

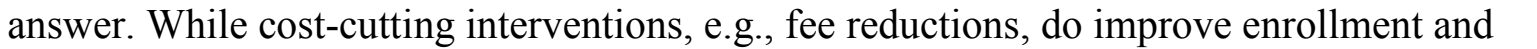

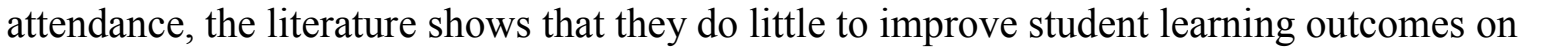

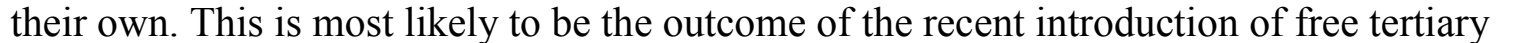

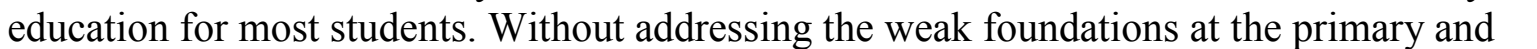

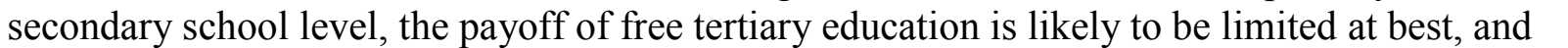

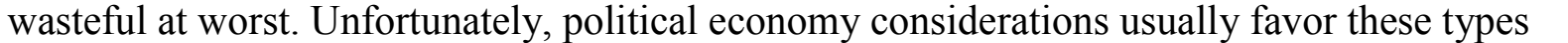
of intervention because they are visible and can be more easily "captured" by politicians. $\square$ $\square$

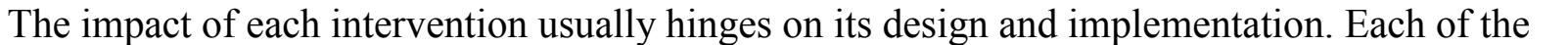

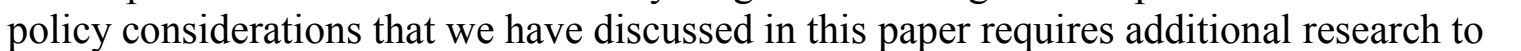

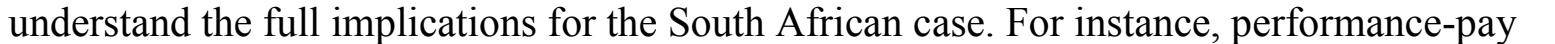

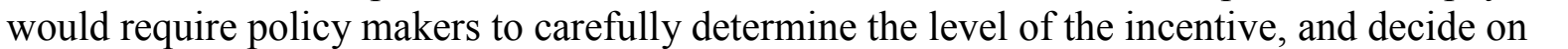

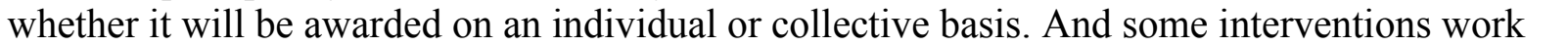

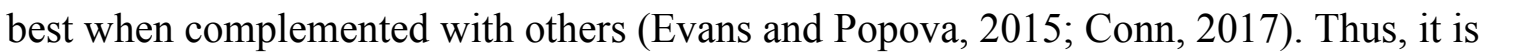

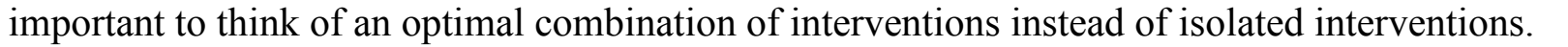

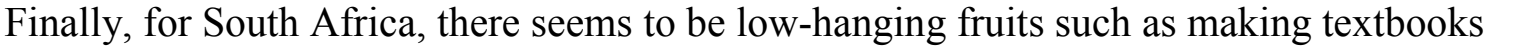

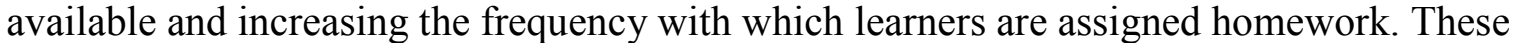

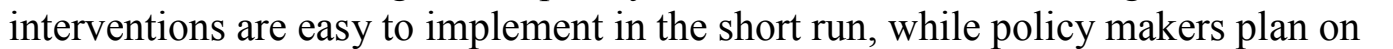

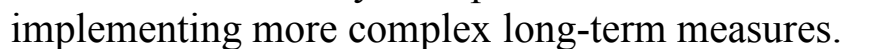

$\square$

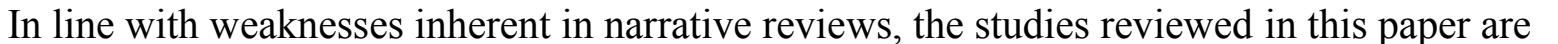

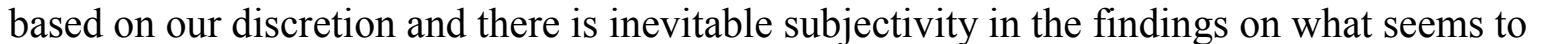

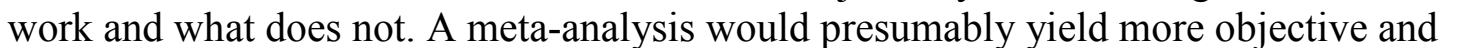

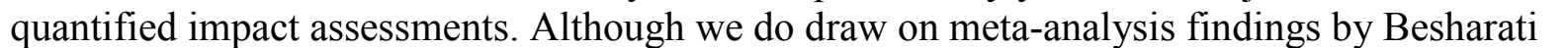

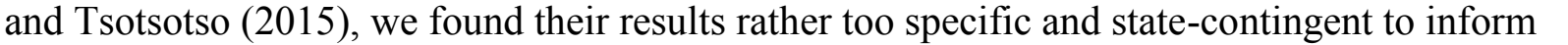

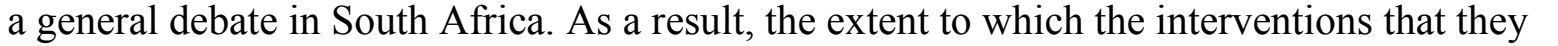

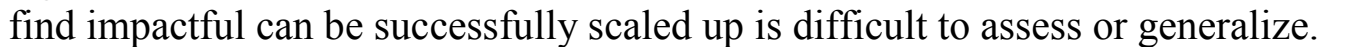
$\square$ 


\section{Appendix I. Data Sources}

\begin{tabular}{|c|c|}
\hline Data & Source \\
\hline 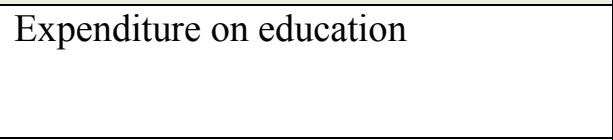 & 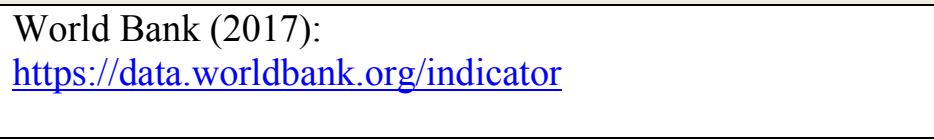 \\
\hline 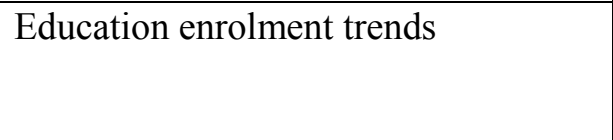 & 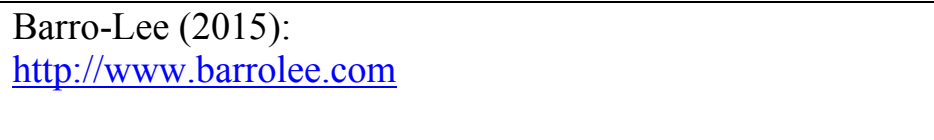 \\
\hline 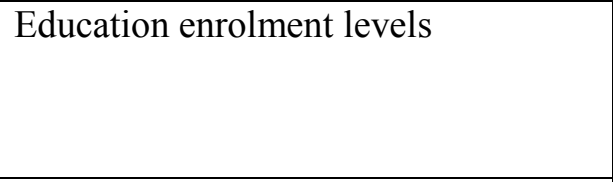 & 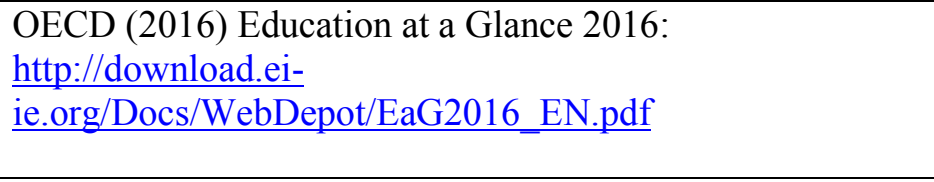 \\
\hline 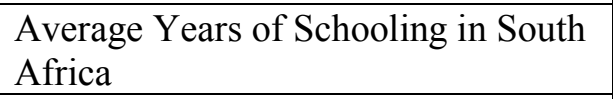 & 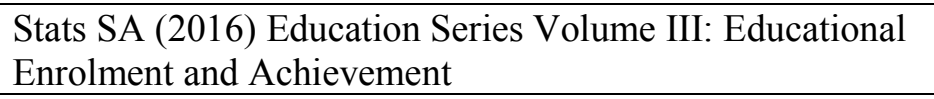 \\
\hline 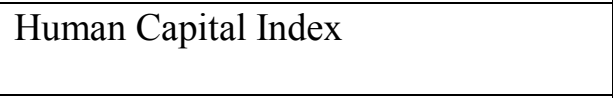 & 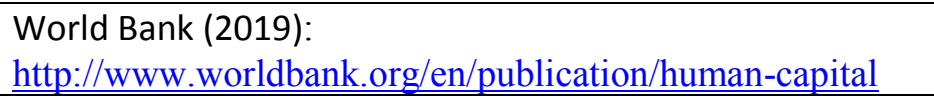 \\
\hline 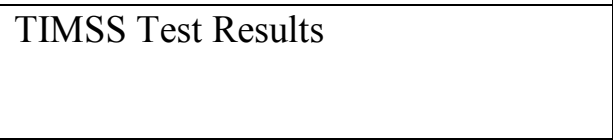 & 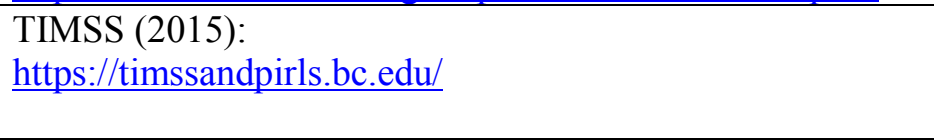 \\
\hline 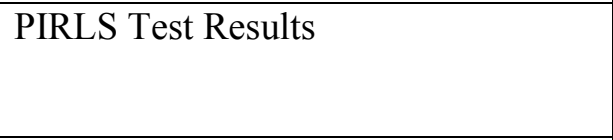 & 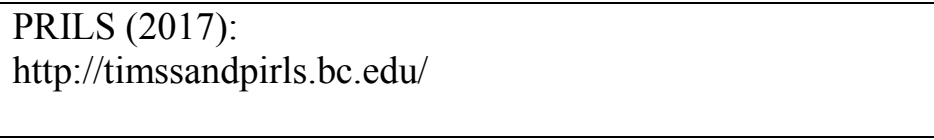 \\
\hline 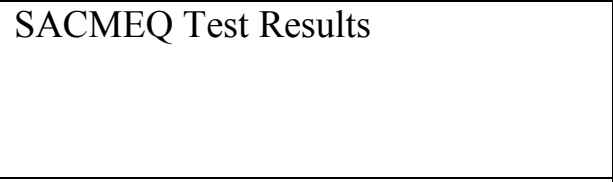 & 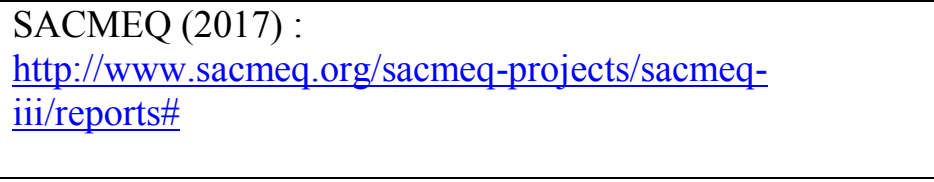 \\
\hline 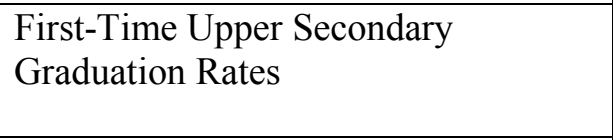 & 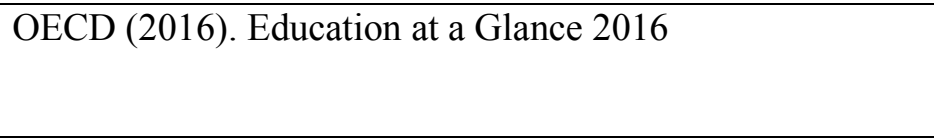 \\
\hline 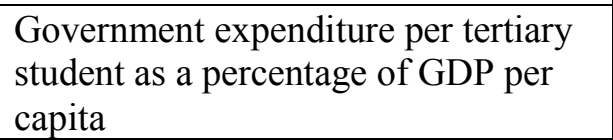 & 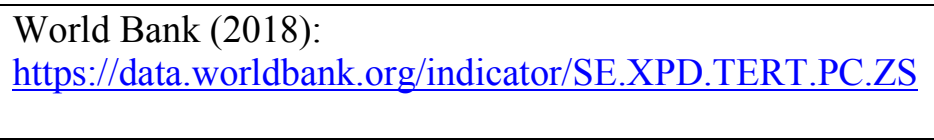 \\
\hline 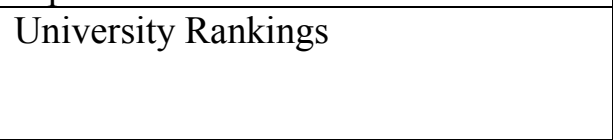 & 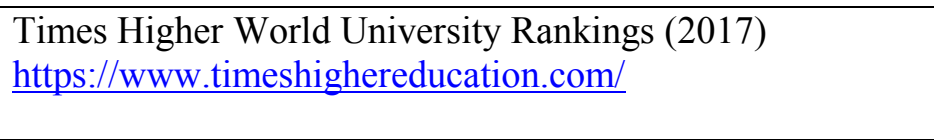 \\
\hline 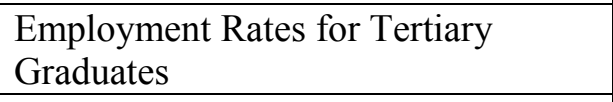 & 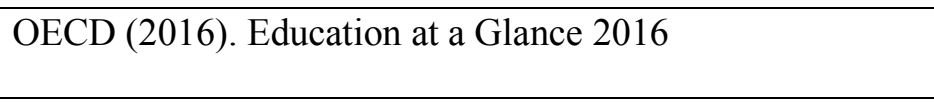 \\
\hline 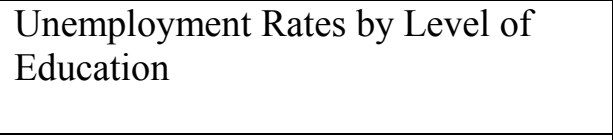 & 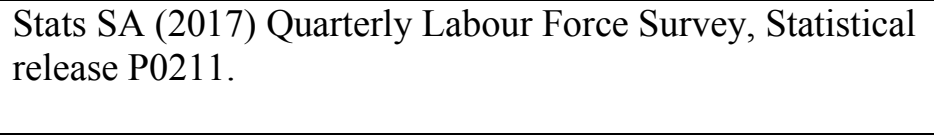 \\
\hline 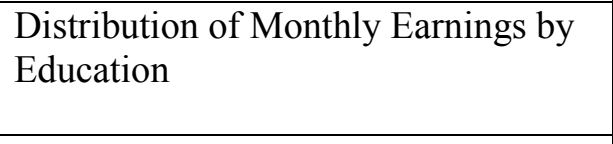 & 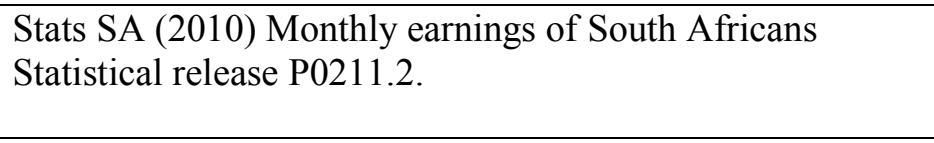 \\
\hline 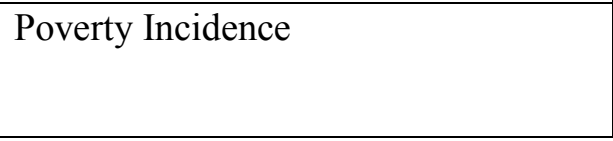 & 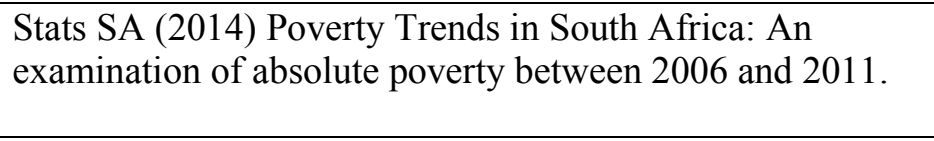 \\
\hline
\end{tabular}




\section{Appendix II. Methodologies for Comparative Metrics}

\section{PIRLS and TIMSS}

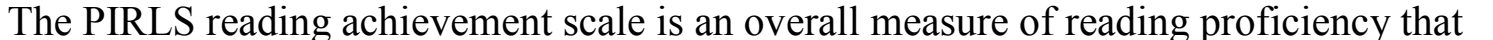

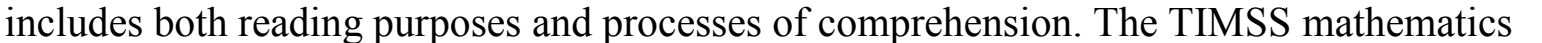

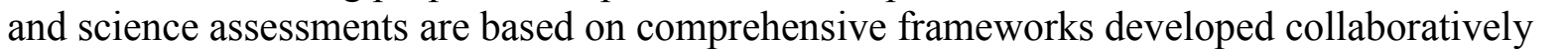

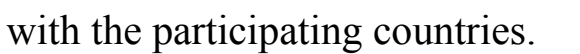

$\square$

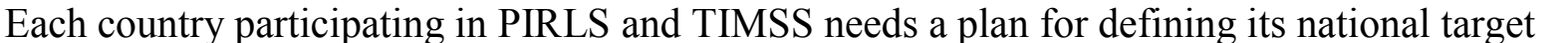

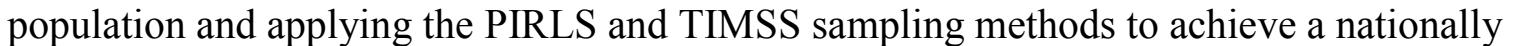

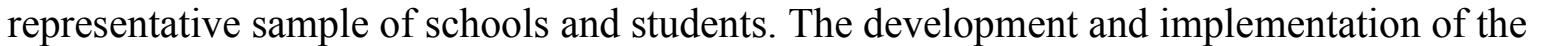
$\square\|\Pi\|$ ampling plan is a collaborative exercise involving the country's National Research

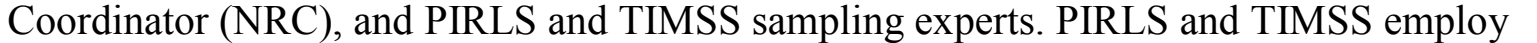

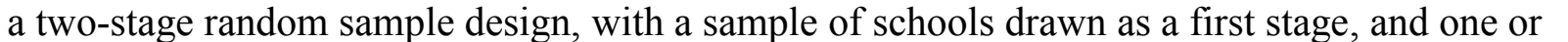

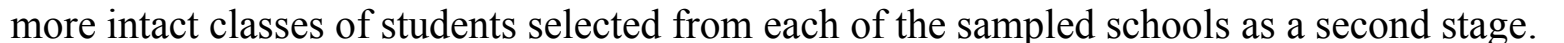

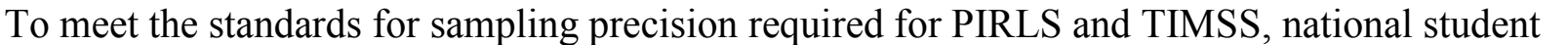

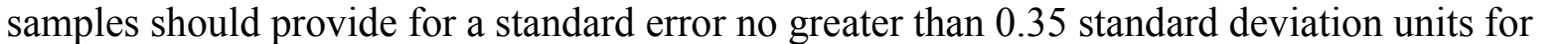

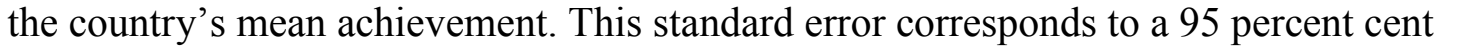

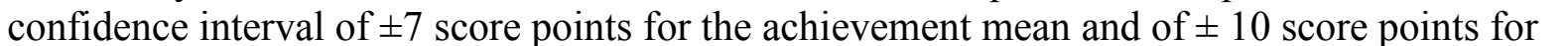

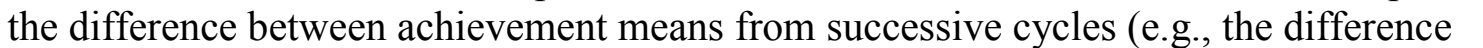

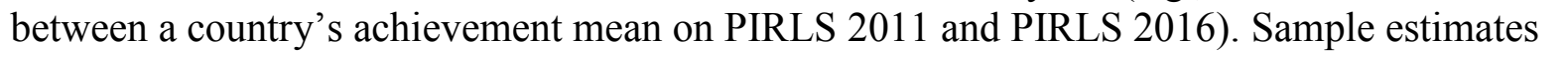

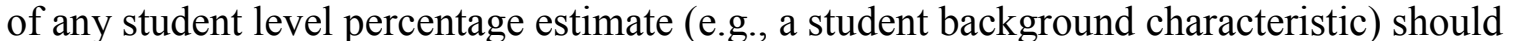

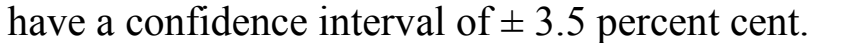

$\square$

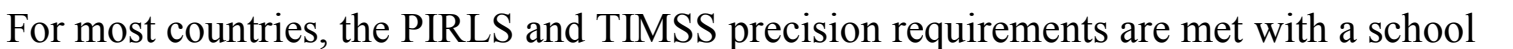

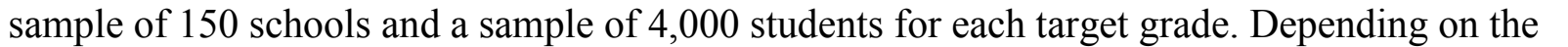

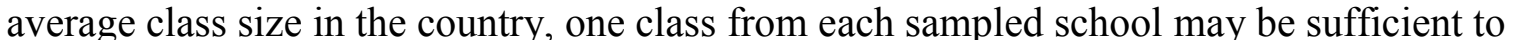

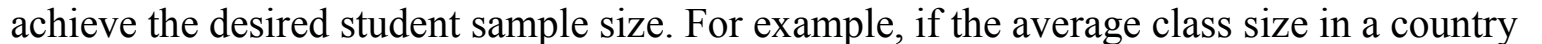

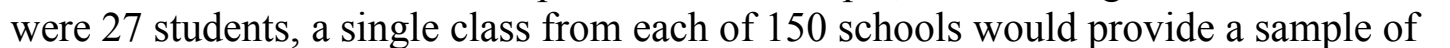

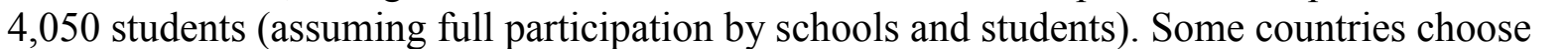
ए

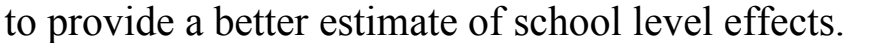

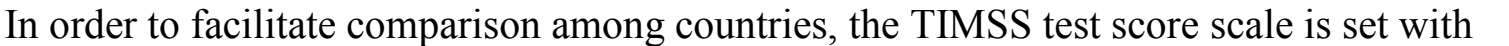

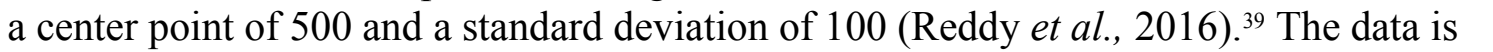

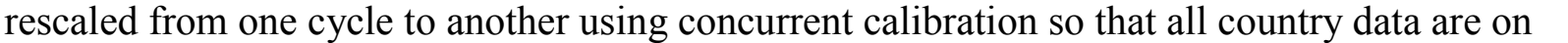

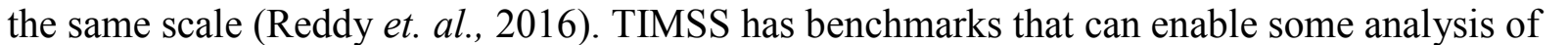

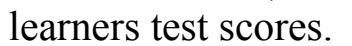

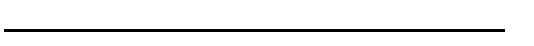

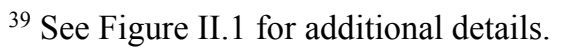




\section{Figure II.1. TIMSS Benchmarks}

$\square$

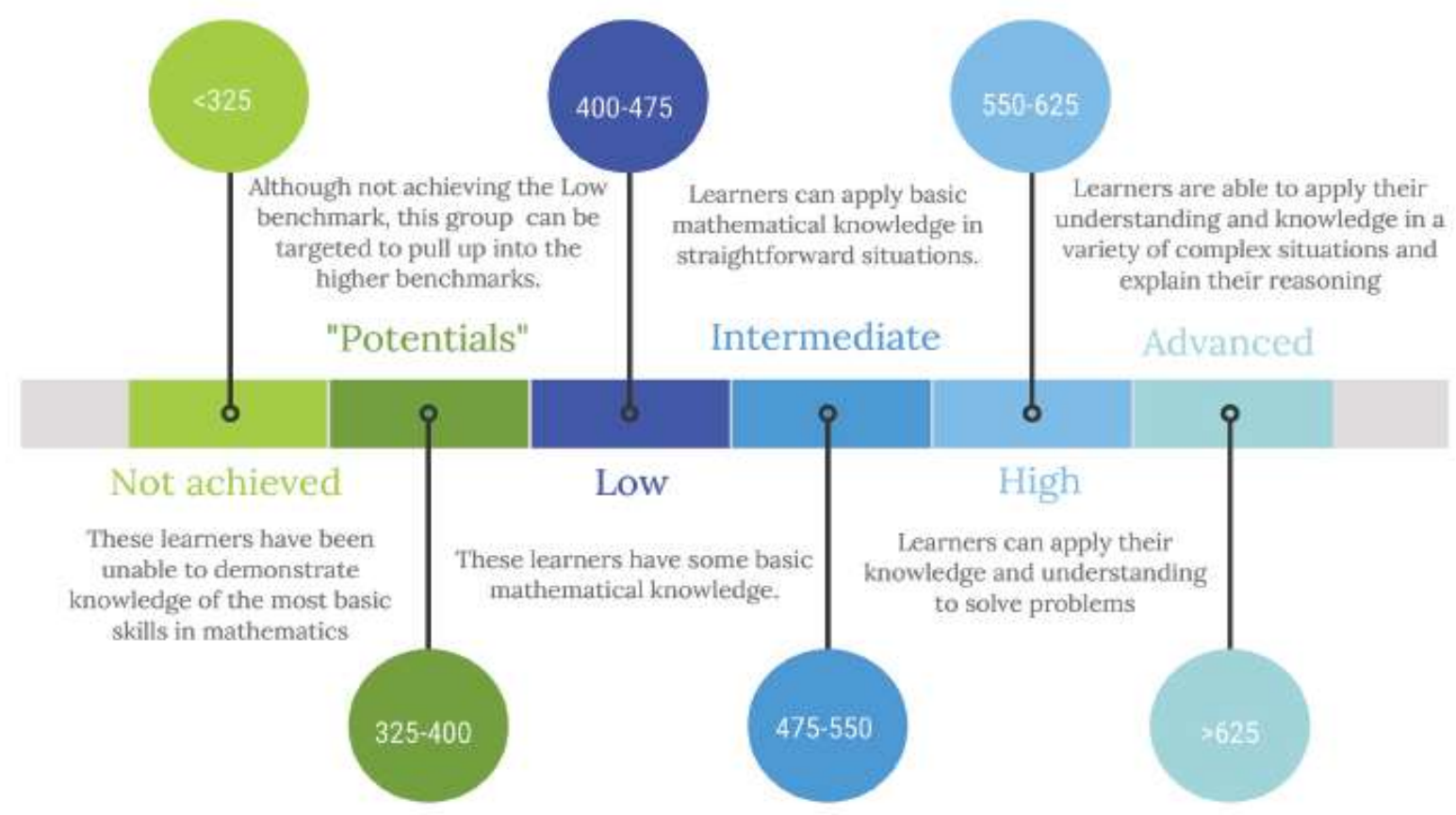

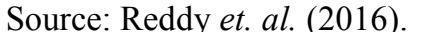

\section{PISA}

$\square$

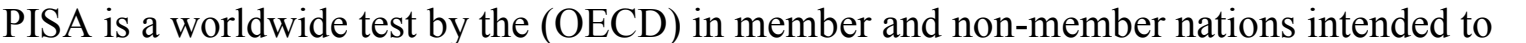

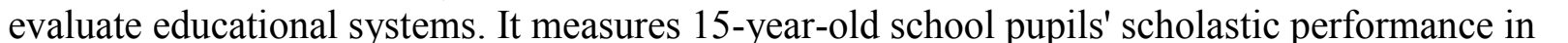

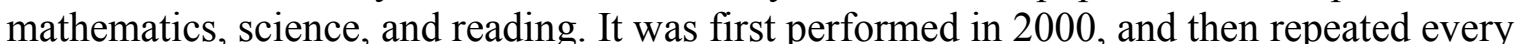
ए

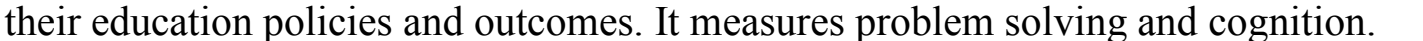

$\square$

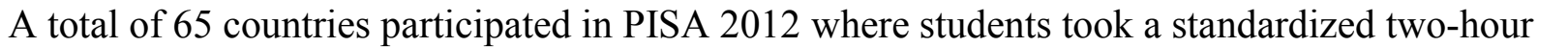

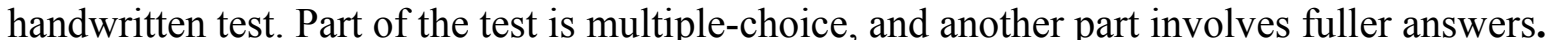

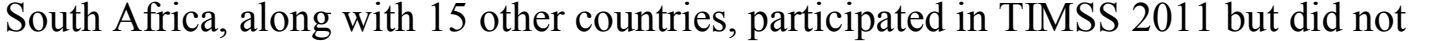

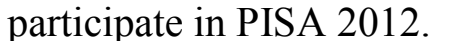

$\square$

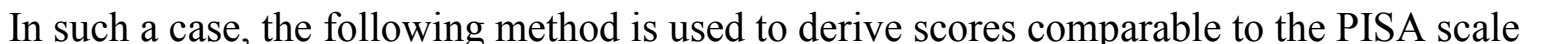

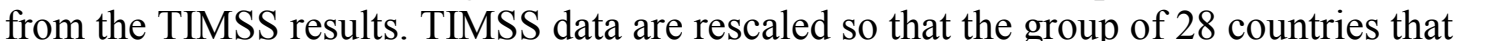

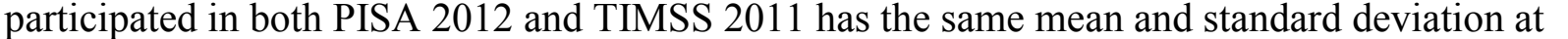

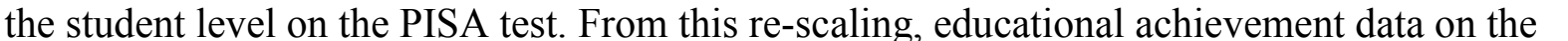

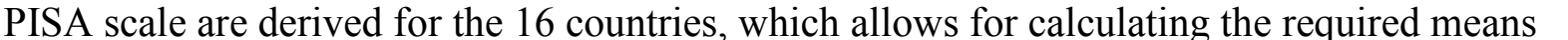

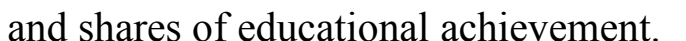




\section{SACMEQ}

The target population is defined as "all pup एणी प

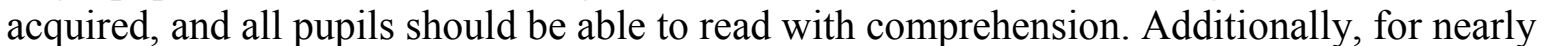

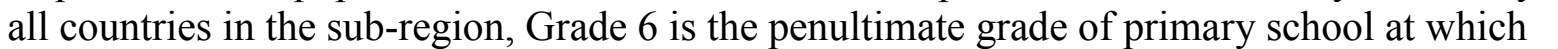

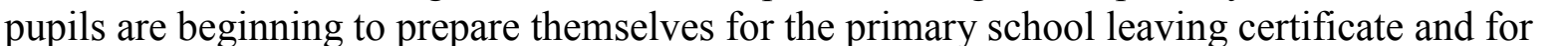

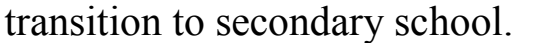

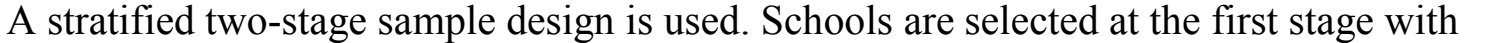

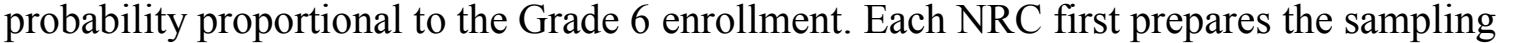
ए

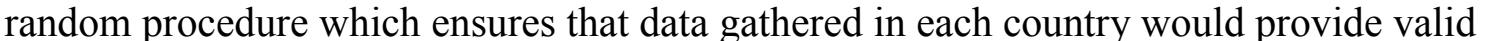

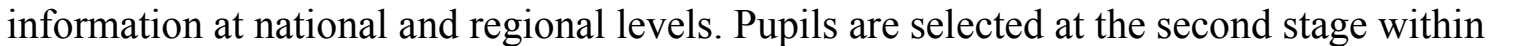

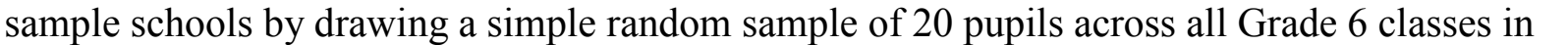

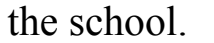

$\square$

\section{The World Bank Human Capital Index}

$\square$

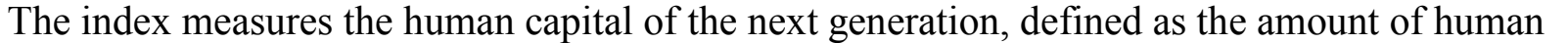

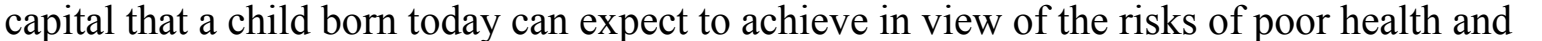

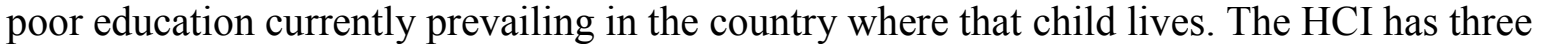

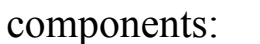

$\square$

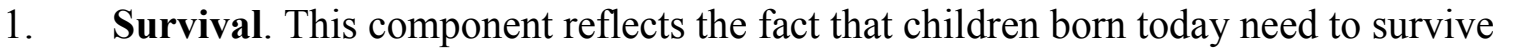

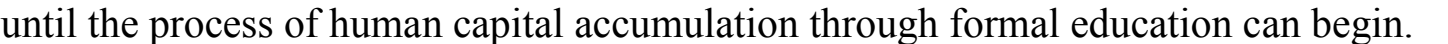

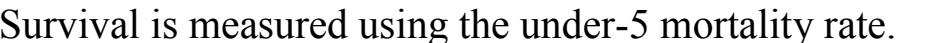
$\square$

$\square \quad$ Expected years of learning-adjusted school

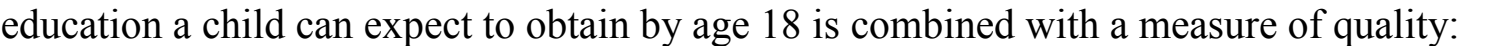
how much children learn in school based on countries' relative performance on

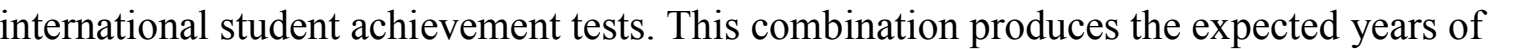

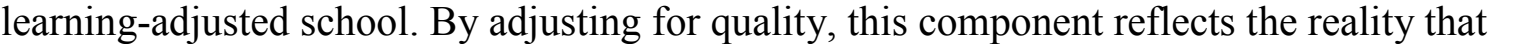

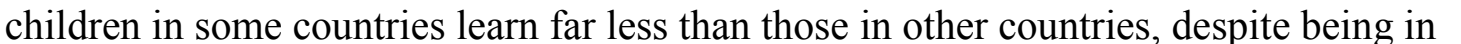

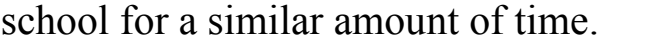

$\square$

$\square \quad$ Health. This component uses two indicators for a country's overall health

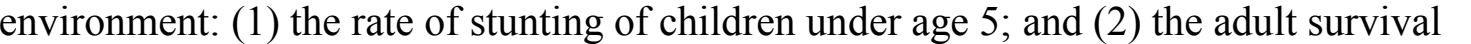

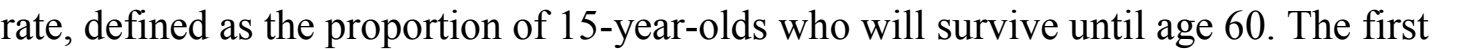

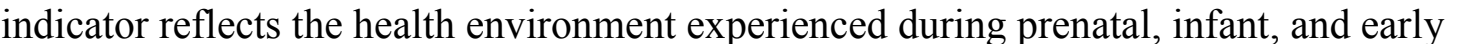

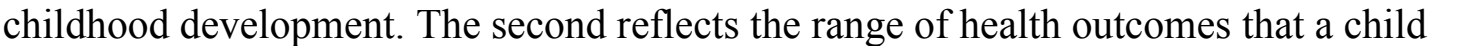

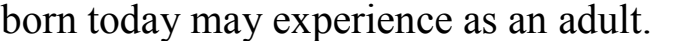

CInternational Monetary Fund. Not for Redistribution 


\section{REFERENCES}

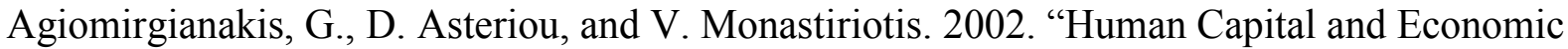
Growth Revisited: A Dynamic Panel Data Study," International Advances in

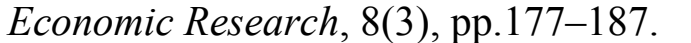

$\square$

Anand, R., S. Kothari, and N. Kumar. 2016. "South Africa: Labor Market Dynamics and

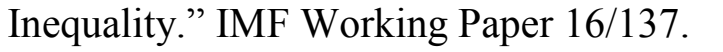

$\square$

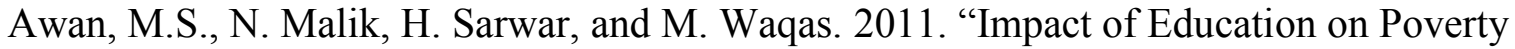

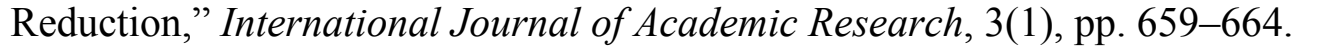

$\square$

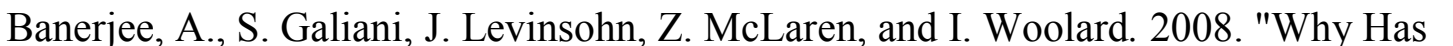

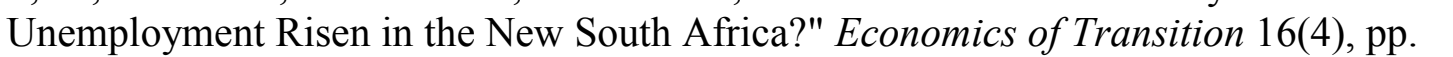
$\square \square-\square \square \square$

$\square$

Barro, R.J., 2013. "Education and Economic Growth" Annals of Economics and Finance, $\square$

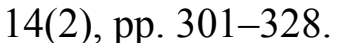

$\square$

Besharati N.A., and K. Tsotsotso. 2015. "In Search for the Education Panacea: A Systematic

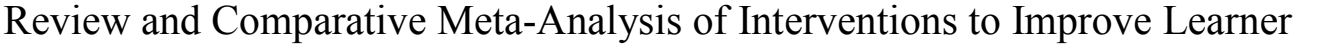

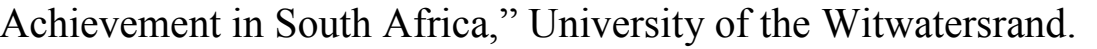

$\square$

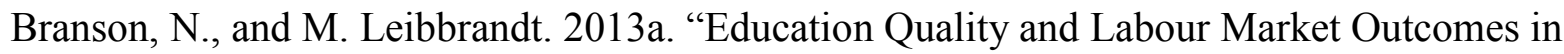

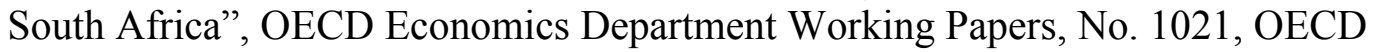

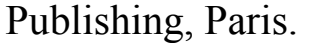

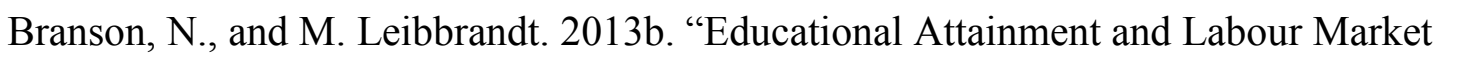

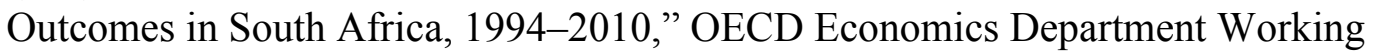

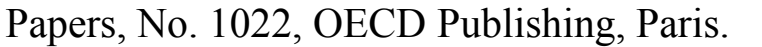

$\square$

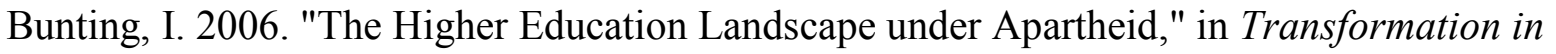

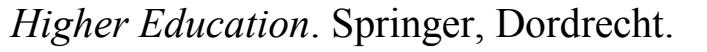

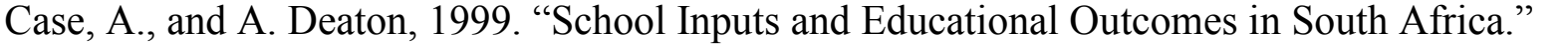

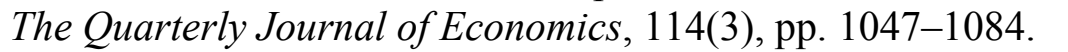

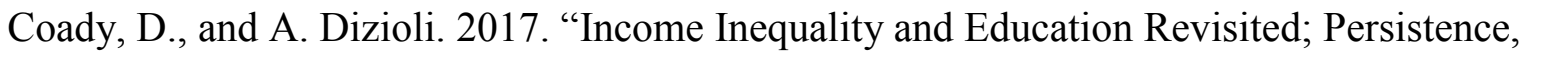

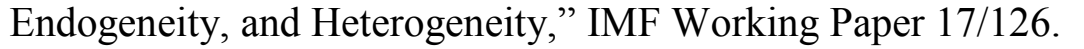

Conn, K.M. 2017. "Identifying Effective Education Interventions in Sub $\square \square\|\|\|\| \square \square \| \square \square \square$ $\square \square \|$ Analysis of Impact Evaluations." Review of Educational Research $\square \square \square \square \square$ $\square \square-\square \square \square$

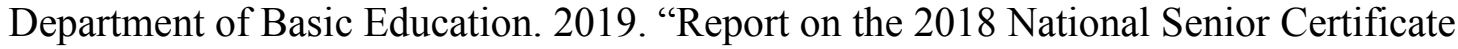

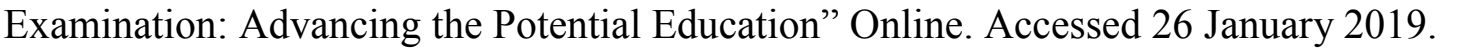

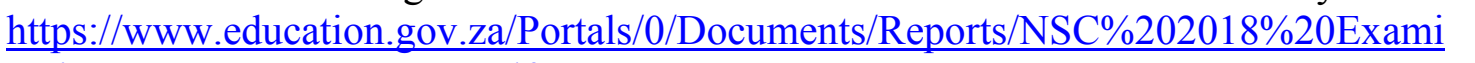

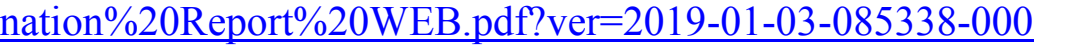

(CInternational Monetary Fund. Not for Redistribution 


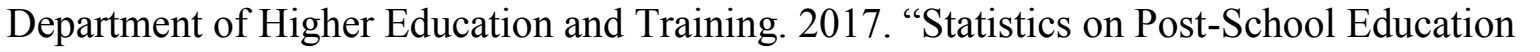

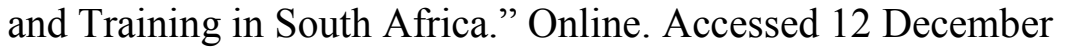

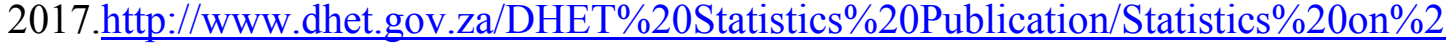
$\underline{\square \square \square|l|}$

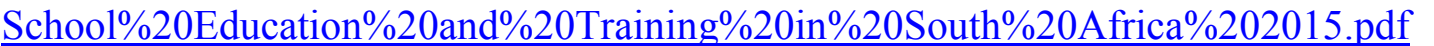

De Ree, J., K. Muralidharan, M. Pradhan, and H. Rogers. 2015. "Double for Nothing?

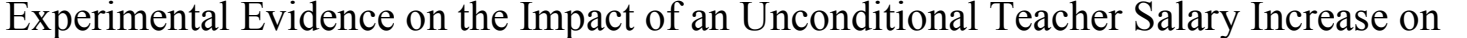

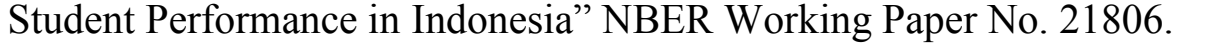

$\square$

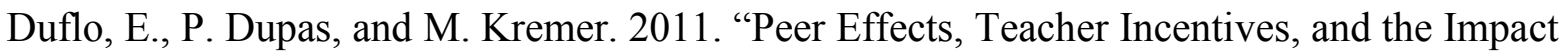

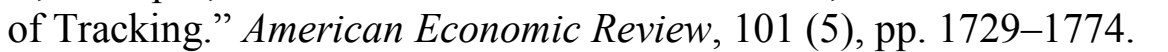

$\square$

Duflo, E., P. Dupas, and M. Kremer. 2012. "School Governance, Teacher Incentives, and

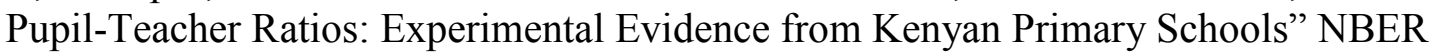

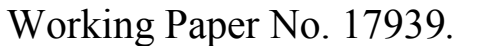

$\square$

Evans, D.K., and A. Popova. 2016. "What Really Works to Improve Learning in Developing countries? An Analysis of Divergent Findings in Systematic Reviews," The World

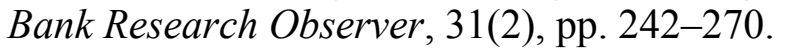

$\square$

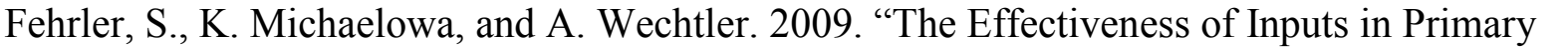

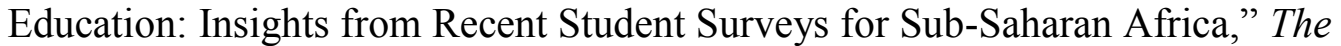

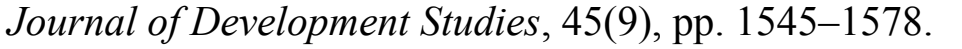

$\square$

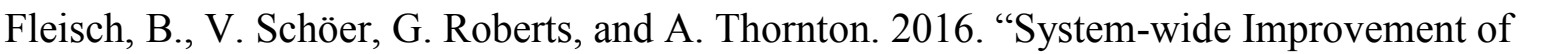

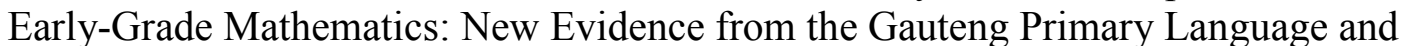

Mathematics Strategy,” International Journal of Educational Development $\square \square$ $\square|\Pi| 1-\square \square$

$\square$

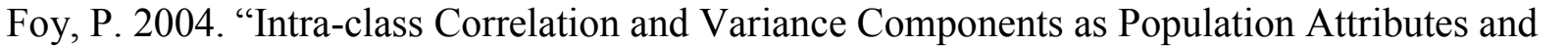

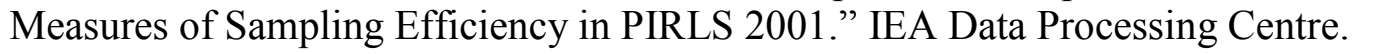
$\square$

Jackson, C.K., R. C. Johnson, and C. Persico. 2015. "The Effects of School Spending on Educational and Economic Outcomes: Evidence from School Finance Reforms," The

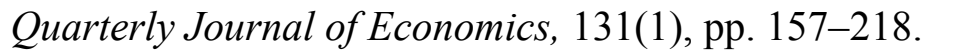

$\square$

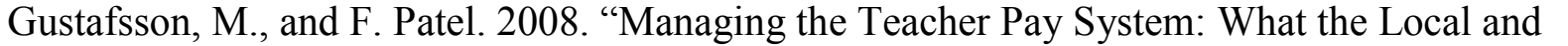

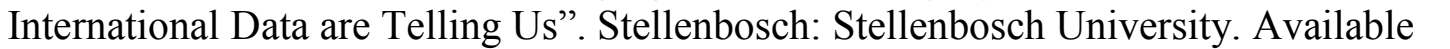

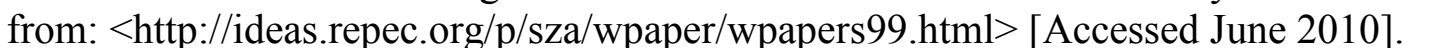

$\square$

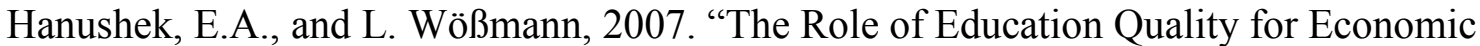

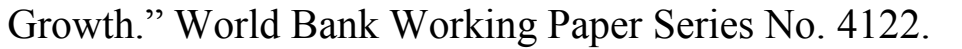




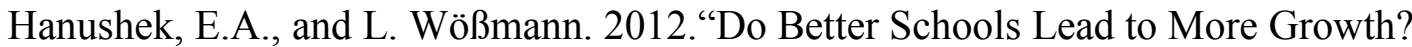

Cognitive Skills, Economic Outcomes, and Causation," Journal of Economic Growth $\square$

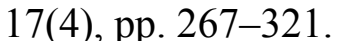

Howie, S.J. 2003. "Language and Other Background Factors Affecting Secondary Pupils' performance in Mathematics in South Africa," African Journal of Research in

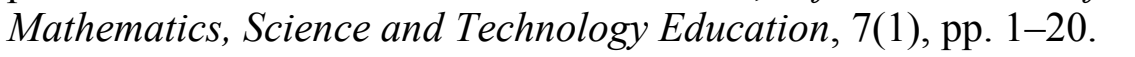

$\square$

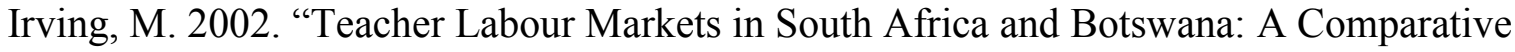

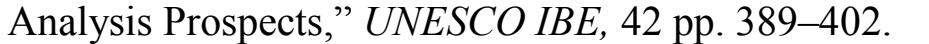

$\square$

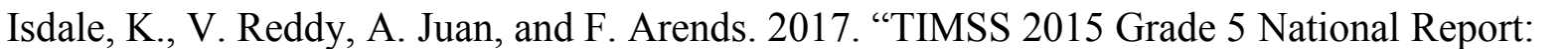

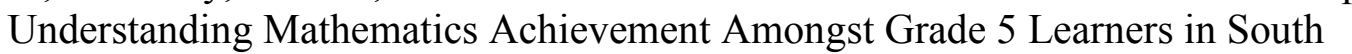

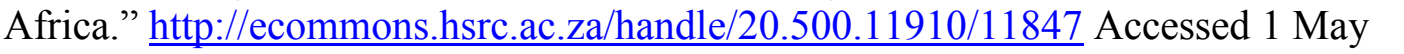
$\square \square \square \square$

Krueger, A.B. and M. Lindahl. 2000. "Education for Growth: Why and for Whom?” NBER $\square \square\|\|\|\| \square \square\|\| \square \square \square$

$\square$

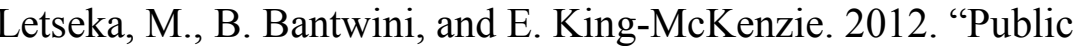

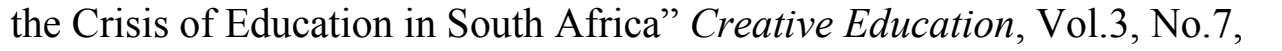

$\square \square \| \square-\square \square \square$

$\square$

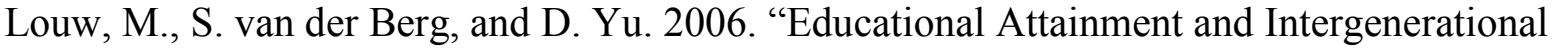
Social Mobility in South Africa," University of Stellenbosch, Stellenbosch Economic

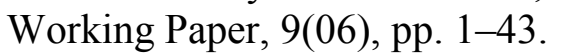

$\square$

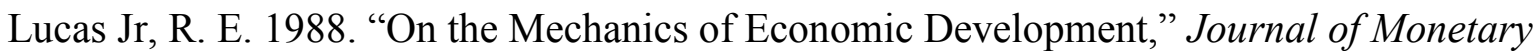

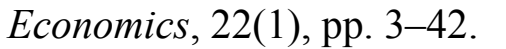

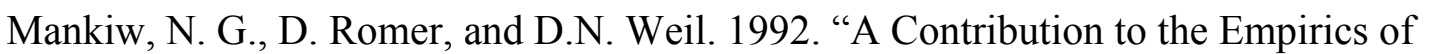

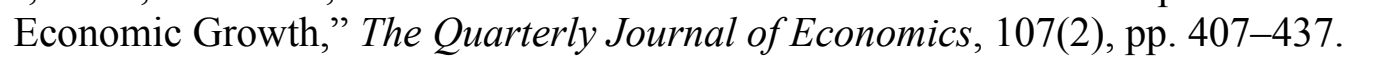

$\square$

Mbiti, I.M. 2016. "The Need for Accountability in Education in Developing Countries," The

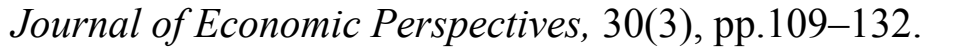

McEwan, P.J. 2015. "Improving Learning in Primary Schools of Developing Countries: A

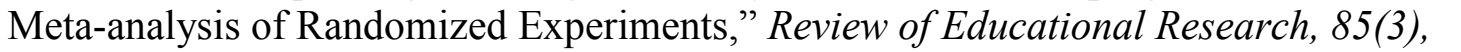
$\square \square \Pi 1-\square \square \square$

Moses, E., S. van der Berg, and K. Rich. 2017. “A Society Divided: How Unequal Education Quality Limits Social Mobility in South Africa,"

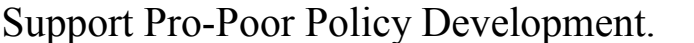

Muralidharan, K. and V. Sundararaman. 2011. “Teacher Performance Pay: Experimental

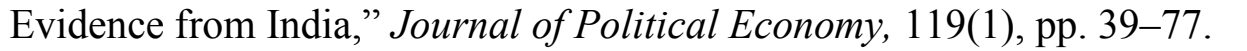


Murray, M. 2016. “Does Poor Quality Schooling and/or Teacher Quality Hurt B1 $\square \square \square \square \square \square \square$

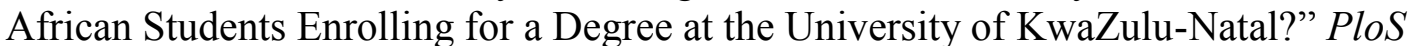
one $\square||||||||||||||||||$

Murnane, R.J. and A. Ganimian. 2014. "Improving Educational Outcomes in Developing

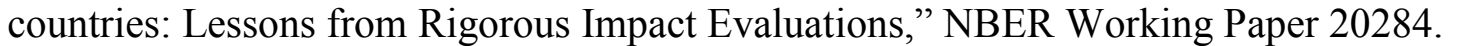

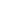

Murtin, F. 2013. "Improving Education Quality in South Africa”, OECD Economics

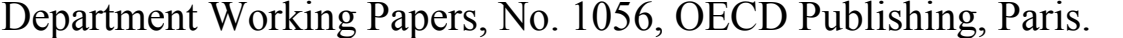

$\square$

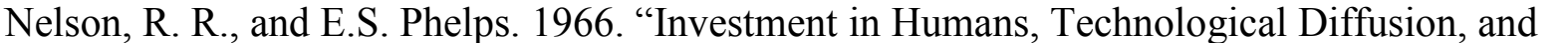

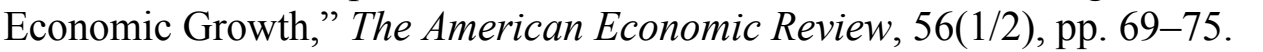

$\square$

OECD. 2015. "Universal Basic Skills: What Countries Stand to Gain.” $\square \square \square \square \square$

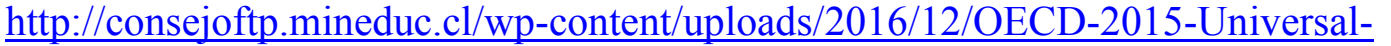

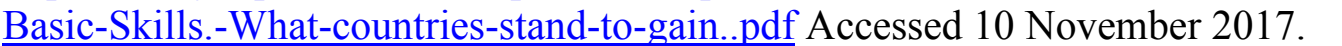

$\square$

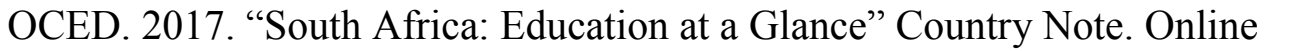

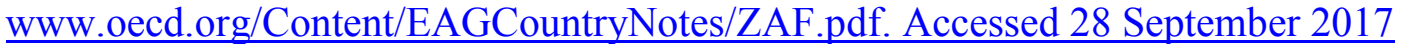

$\square$

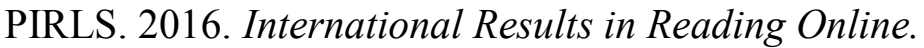

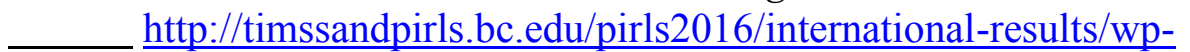

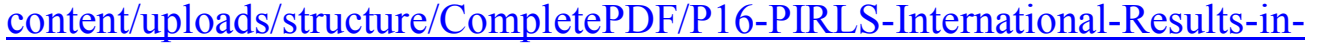

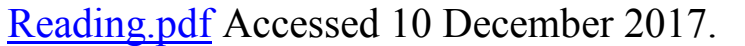

$\square$

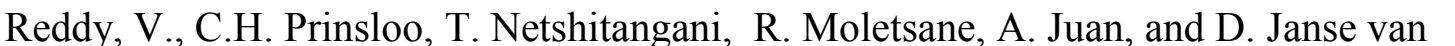

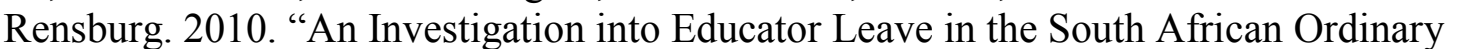

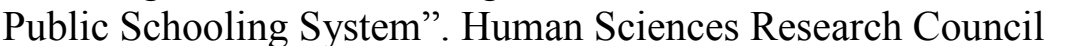

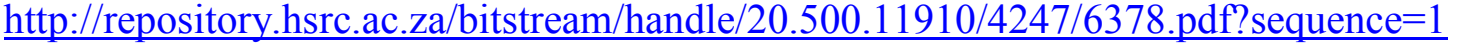

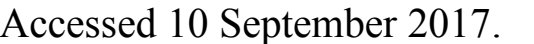

$\square$

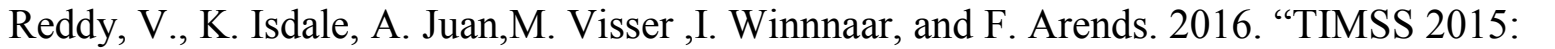

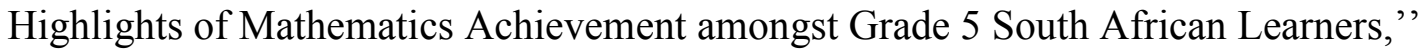

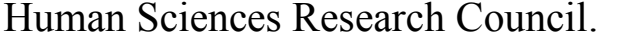

$\square$

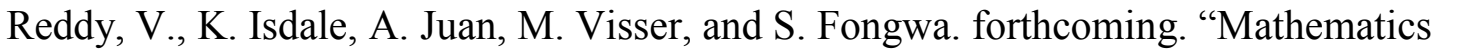

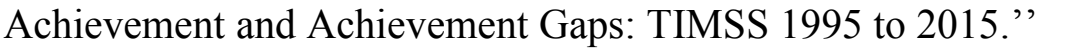

$\square$

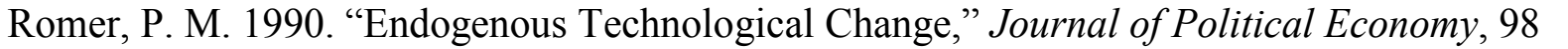

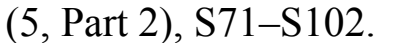

$\square$

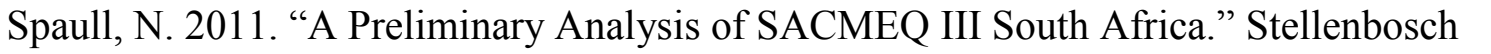

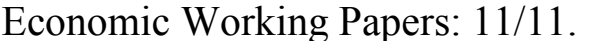

$\square$

Spaull, N. 2013. "South Africa's Education crisis: The Quality of Education in South Africa

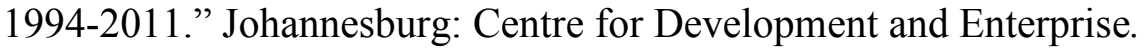

(CInternational Monetary Fund. Not for Redistribution 


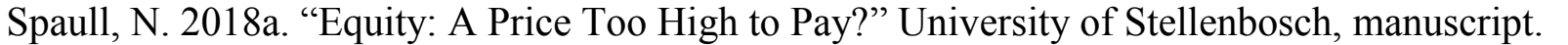

$\square$

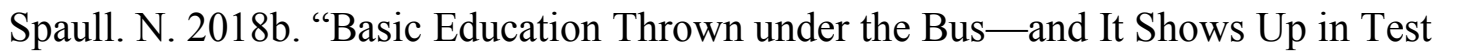

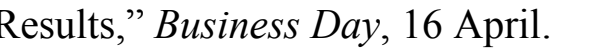

प1]

Spreen, C. A. and V. Salim. 2006. "Education Rights, Education Policies and Inequality in South Africa" International Journal of Educational Development Volume $26 \square \square \| \square \square$ $\square \square \square-\square \square \square$

$\square$

University of the Witwatersrand. 2016. "Report of the University Panel of Funding Model(s) for Higher Education in South Africa. ”

$\square$

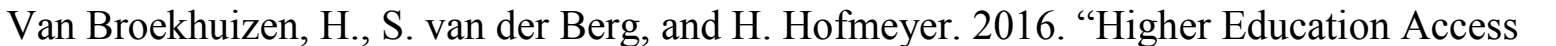

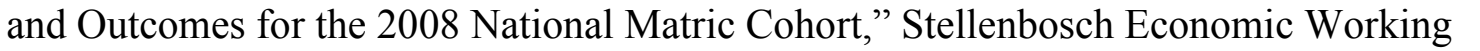

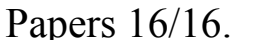

$\square$

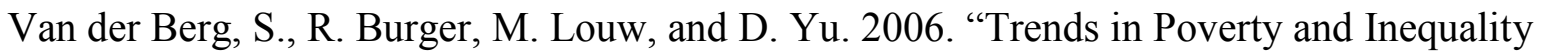

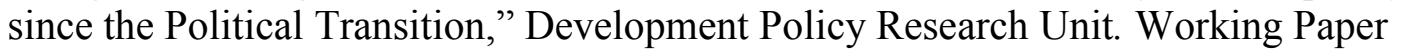
$\square \square \square \square$

$\square$

Van der Berg, S. 2008. "How Effective are Poor Schools? Poverty and Education $\square \square \square \square \square \square \square \square$

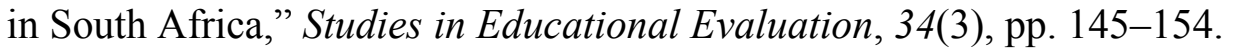

$\square$

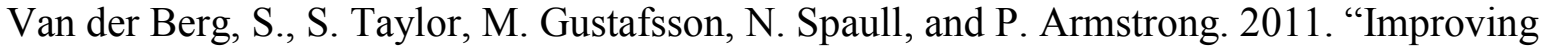

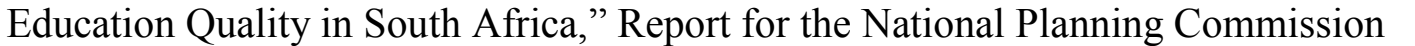

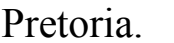

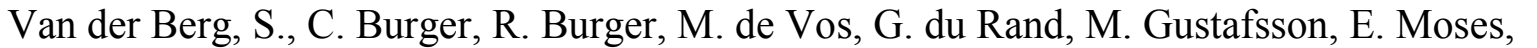

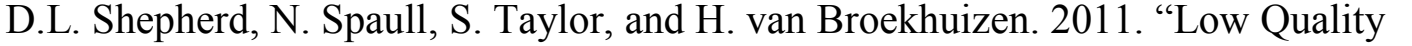

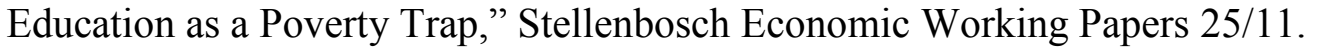 $\square$

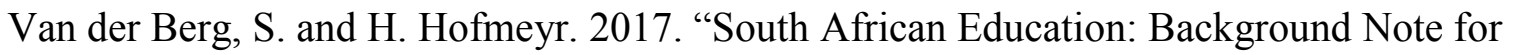 World Bank’s South African Country Diagnostic " Research on Socio $\square \square \square \square \square \square$

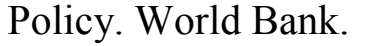

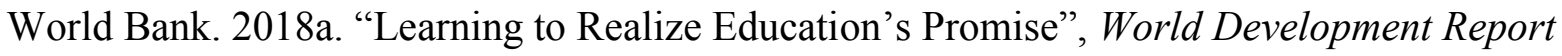
$2018 \square \square \square \square\|ा\| ा \| \square \square$

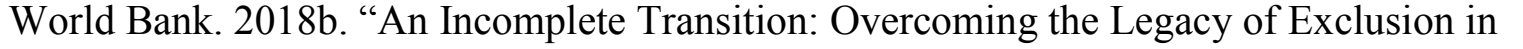

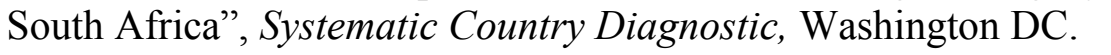

$\square$

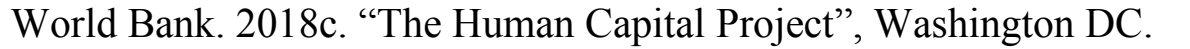

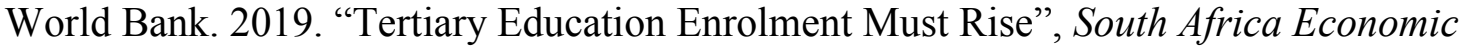
Update No.12, $\square \square \| \square|\Pi| \square \square \square$

$\square$

(CInternational Monetary Fund. Not for Redistribution 


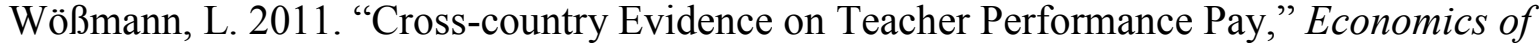

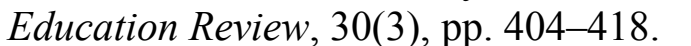

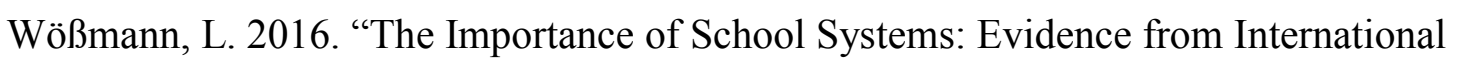

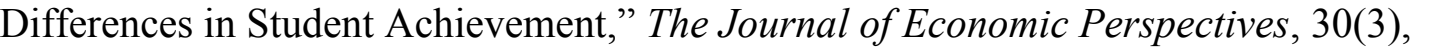
$\square \square+\square-\square \square$

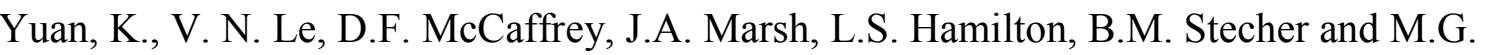

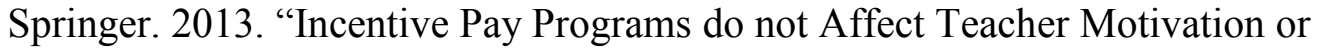

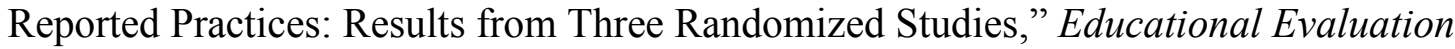

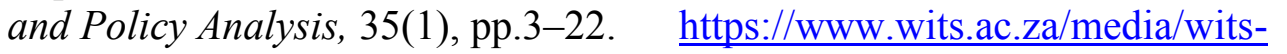

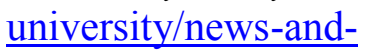

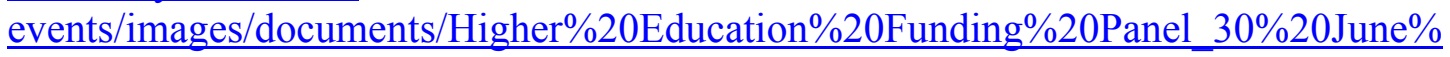

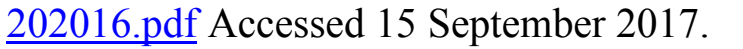

